\title{
Millimeter Wave and Microwave Instrumental Development for Analytical and Astrochemical Applications
}

\author{
Amanda Lee Steber \\ Mattoon, Illinois
}

B.S. (Chemistry), Eastern Illinois University, 2009

B.S. (Biology), Eastern Illinois University, 2009

A Dissertation presented to the Graduate Faculty

of the University of Virginia in Candidacy for the Degree of

Doctor of Philosophy

Department of Chemistry

University of Virginia

December 2014 


\section{Abstract}

Advances in digital electronics have allowed several fields to upgrade their existing instrumentation. These upgrades allow for more sensitive and rapid data acquisition. Taking advantage of these faster and in some cases cheaper electronics, new instrumentation in the millimeter wave and microwave regime have been developed and are presented in this thesis. A chirped pulse-Fourier transform millimeter wave spectrometer operating between 260-295 GHz was developed for the purpose of analytical and astrochemical applications. This instrument was further refined with the addition of a pulsed discharge nozzle to measure reactive species at these frequencies using the segmented chirped pulse-Fourier transform millimeter wave technique. Both instruments have the ability for high sensitivity measurements and improvements in speed of acquisition and automation have been implemented.

Besides the chirped pulse-Fourier transform millimeter wave instrument, cost reduction of both the millimeter wave instrument and current microwave instruments was investigated in this thesis. Many of the expensive digital electronics were replaced with monolithic microwave integrated circuits, and the performance of these instruments was investigated both at 260-295 GHz and 18-26 GHz. The applications of these instruments, for both analytical techniques and astrochemistry, are a main theme throughout this thesis. By applying these instruments towards the problems facing both fields, and by developing new strategies for astrochemical analysis, a new and better understanding of the complex chemistry occurring in the interstellar medium and terrestrially will evolve. 


\section{Table of Contents}

Chapter 1: Introduction.........................................................1

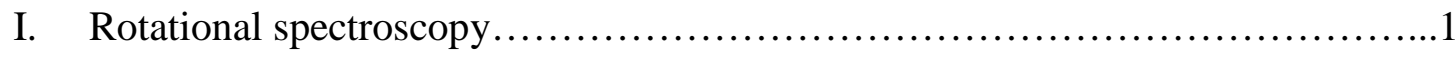

II. Spectroscopic techniques and applications................................

III. Instrument and astrochemical technique development.........................9

Chapter 1 References...............................................13

\section{Chapter 2: An Arbitrary Waveform Generator Based Chirped Pulse Fourier}

Transform Spectrometer Operating from 260-295 GHz.........................18

I. Introduction.......................................................... 18

II. Experimental.......................................................22

a) Chirped-pulse excitation source.......................................22

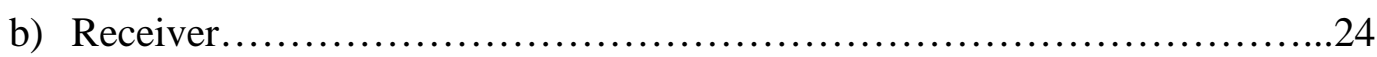

c) Single- and dual-channel spectrometer designs........................25

d) Sample chamber..................................................27

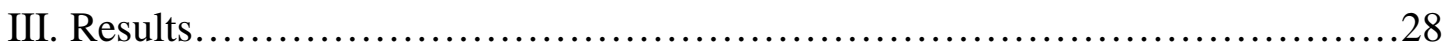

IV. Discussion........................................................ 38

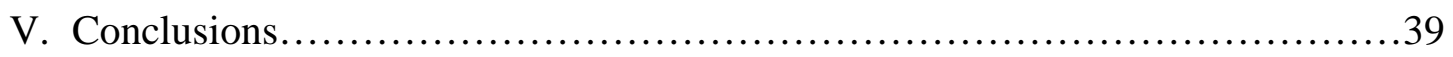

Chapter 2 References...............................................41

Appendix A............................................................ 44

Chapter 3: A Two-Color, Time-Domain Spectrometer for Fourier Transform mm-

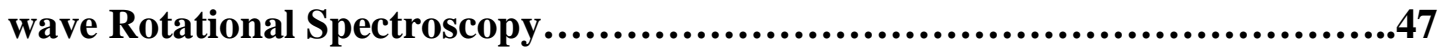

I. Introduction.......................................................47 
II. Experimental.......................................................49

a) Spectrometer design..........................................49

b) Implementation for Fourier transform spectroscopy ...................54

III. Results........................................................... 58

a) Variable pulse duration measurements to characterize the transition moment.......................................................60

b) Double-resonance spectroscopy and enhanced chemical selectivity in gas analysis.....................................................63

c) Pulse echoes and collisional relaxation...........................69

d) Population recovery and collisional relaxation.........................74

IV. Discussion.......................................................76

V. Conclusions....................................................... 79

Chapter 3 References................................................81

Chapter 4: A Design of a Low-Cost Microwave Spectrometer Operating in the 18-26

GHz Frequency Range..........................................................83

I. Introduction....................................................... 83

II. Experimental...................................................... 85

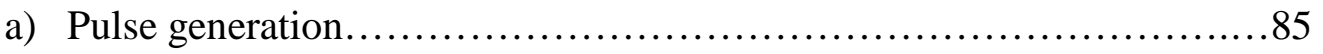

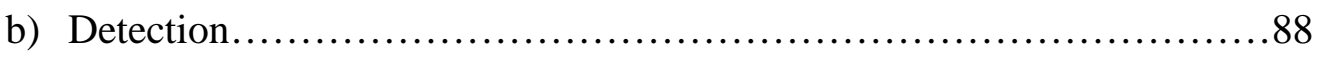

c) Sample chamber...............................................88

d) Graphical user interface......................................90

III. Results.........................................................92 


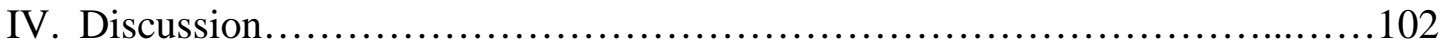

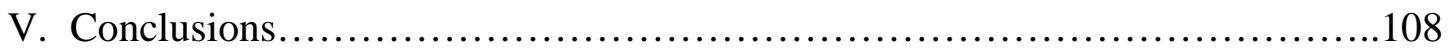

Chapter 4 References........................................................109

Chapter 5: A Chirped Pulse - Fourier Transform Millimeter wave Discharge

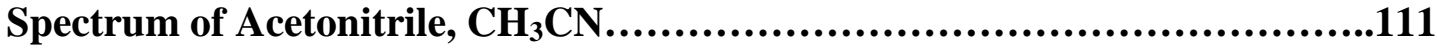

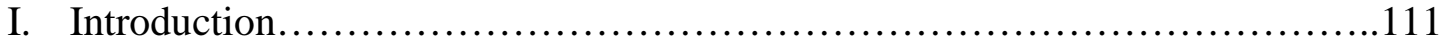

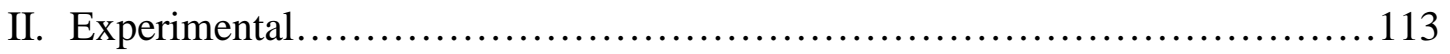

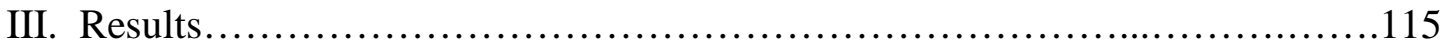

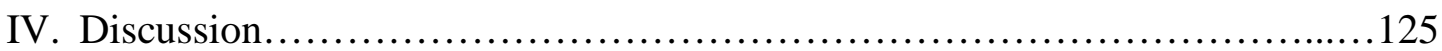

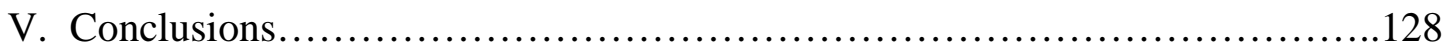

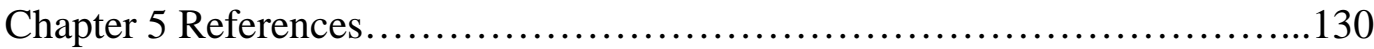

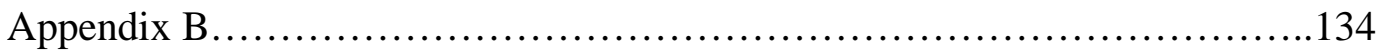

Chapter 6: A Theoretical and Observational Study of the Formational Pathway of

Cyanomethanimine in Sagittarius B2............................................139

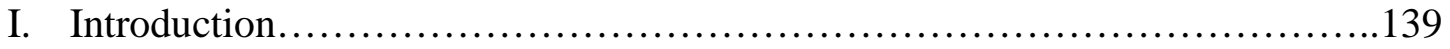

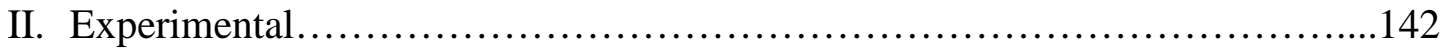

a) $\mathrm{Ab}$ initio calculations..............................................

b) Observational details............................................ 144

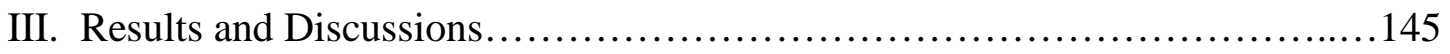

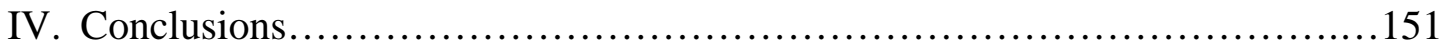

Chapter 6 References.................................................153 
Chapter 7: The Development of the Spatial Distribution Screening Method and Its

Applications to ALMA Datasets............................................155

I. Introduction........................................................ 155

II. Experimental....................................................... 158

III. Source information...............................................159

IV. Results............................................................... 161

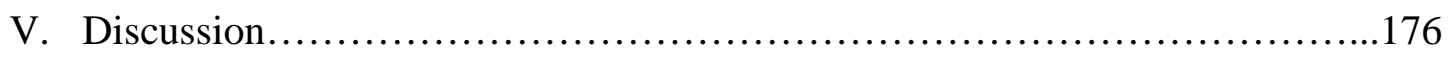

VI. Conclusions.....................................................178

Chapter 7 References...............................................180

Chapter 8: Conclusions..........................................................183

I. Summary......................................................... 183

II. Future developments............................................. 184

Chapter 8 References..................................................187 


\section{Acknowledgements}

A lot goes into the making of a Ph.D. thesis, in terms of both time and support. I am where I am today because of the incredible support system that I have had throughout my time at UVa. This journey would never have started if it weren't for my undergraduate advisors, Rebecca and Sean Peebles. During my time at EIU, they guided me and taught me several fundamental principles that I live by even now. Their door was always open, and they provided an engaging and fun place to learn. I will forever be indebted to them for the kindness that they have shown me and the support that they have given me throughout the years.

I also would not be where I am today if it were not for the many lab mates that I have had during my time here. I have been lucky enough to learn from and alongside them. The Pate group members have included Matt Muckle, Daniel Zaleski, Simon Logsiger, Nathan Seifert, and Luca Evangelisti as well as various undergraduate students who helped to enrich my time here. There are two members of the lab who have continually been there either for moral support or just as a really great friend and they are Justin Neill and Brent Harris. Without the two of them, my journey here would have been much more lonesome and dark.

Another individual who deserves recognition is my advisor, Brooks Pate. He has made everything that I have accomplished here possible and has instilled countless skills that I will use for the rest of my life. His critical thinking and scientific assessment is something that I strive to be able to do. He is truly an innovator who teaches us to strive 
for the best as he will settle for nothing less. I can only hope to make even a fraction of the impact during my career that he has made.

The last group of individuals who deserve my eternal thanks and gratitude is my family and friends. Without them, I would be truly lost. While all of my friends deserve some kind of recognition, this journey would definitely not have been possible if it weren’t for my dear friend Briony. She was always there for me, and our time watching Homeland was irreplaceable. My parents have always encouraged me to pursue whatever endeavor I chose, and have always been there to support me during the rough times. My siblings have also been a fountain of support throughout the years. I am grateful that they are willing to put up with me. Last but not least is my loving husband, Cristóbal. He has endured quite a bit over the last few years, and yet he has always been there with a helping and loving hand to lift me back up when life seemed impossible. As we embark on the next chapter of our lives, I know we will be able to conquer anything. 


\section{Chapter 1}

\section{Introduction}

\section{Rotational spectroscopy}

Rotational spectroscopy is a highly sensitive method used for chemical characterization and analysis ${ }^{1,2}$. The technique requires that the sample of interest be in the gas phase and in most cases also have a permanent dipole moment. The rotational spectrum, or fingerprint, of the molecule that arises from the interaction of the molecular sample with electromagnetic radiation comes from the quantization of the molecule's angular momentum. Depending upon the system's temperature and the sample's molecular shape, the spectrum will be observed in the microwave or millimeter wave (mm-wave) regime based on the Maxwell-Boltzmann distribution of the rotational states. The interaction of the molecule with the electromagnetic radiation of the system causes the molecule's rotational energy to change, according to the Bohr condition $(\Delta E=h v$, where $h$ is Planck's constant). When the molecule interacts with radiation of the correct frequency, a quantum-mechanically allowed transition occurs and gives rise to a line corresponding to that frequency in the spectrum.

These lines, or transitions, and the transition intensities are related to structural and electronic properties of the molecule that can be calculated. Even slight changes in the geometry of the molecule give rise to a different chemical fingerprint. These changes are due to the shifts in the center of mass of the molecule, which is related to the molecule's moment of inertia. The transitions of the molecule are fit against frequencies of the molecule predicted by an effective Hamiltonian, and the rotational constants of the 
molecule are obtained. In the rigid rotor approximation, the rotational constants (in $\mathrm{Hz}$ ) are inversely proportional to the molecule’s moment of inertia:

$$
A=\frac{h}{8 \pi^{2} I_{a}}, \quad B=\frac{h}{8 \pi^{2} I_{b}}, \quad C=\frac{h}{8 \pi^{2} I_{c}}
$$

where $a, b$, and $c$ denote the principal axes of the molecule. These axes are defined so that the off-diagonal terms of the inertial tensor are zero. These follow the convention $A \geq B \geq C$

The rotational constants would be good enough to describe the structure of a molecule for a simple case in which the rigid rotor approximation was valid. However, molecules are not completely rigid, and therefore, are subject to distortions from vibrational motions and, consequently, centrifugal distortion. The Hamiltonian can be expanded to include parameters that help to describe these motions, as well as nuclear or electronic spins that couple with the rotational angular momentum. Molecules can also have internal motion, such as a methyl rotor torsion, which can often be treated pertubatively in the rotational Hamiltonian. All of these effects influence the line intensity, splitting, and transition pattern that are seen in the spectrum.

The intensities of the molecular transitions give information about the concentration of the molecule of interest in a sample. They depend on the magnitude and direction of the permanent dipole that is required for rotational spectroscopy. Depending upon the geometry of the molecule, there can be a non-zero dipole component $\left(\mu_{\mathrm{x}}\right)$ along each of the principal axes. For an asymmetric top (i.e., a molecule where $A \neq B \neq C$ ), $a$-type, $b$-type, and $c$-type transitions may be observable. For a type of transition to occur, it is necessary that $\mu_{\mathrm{x}} \neq 0$, where $\mathrm{x}=a, b$, or $c$. In the weak pulse limit, the intensity of a 
transition scales with $\mu^{2}$; however, in conditions of optimal transition excitation $(\pi / 2$ conditions), the intensity of the transition only scales as $\mu$. The magnitude of the dipole moment can be measured through Stark effect measurements. Another way to measure the dipole moment is with an instrument in which the electric field has been characterized. The dipole moment is measured through obtaining the nutation curve of the molecule. A nutation measurement ${ }^{3,4}$ determines the relationship between the signal intensity and the pulse duration. It is a measurement of the Rabi frequency of a two-state resonance within a molecule and the general relationship to the dipole moment is given $\mathrm{as}^{5}$

$$
\omega_{R}=\frac{\overrightarrow{M_{10}} \cdot \vec{E}}{\hbar}
$$

in which $\omega_{\mathrm{R}}$ is the Rabi frequency, $\mathrm{M}_{10}$ is the transition dipole matrix element, and $\mathrm{E}$ is the electric field. The Rabi frequency corresponds to the pulse duration with maximum intensity, which is also the $\pi / 2$ pulse. In the situation where a $\pi / 2$ pulse is applied to the system, the ratio of populations in the lower and upper states is one. In knowing the dipole moment from this calculation and the optimal pulse duration, characterization of an unknown molecule can begin to take place. Measurements determining the collisional and lifetime decay rates through Hahn echoes ${ }^{6}$ and saturation recovery methods ${ }^{7,8}$ help with mass analysis of the unknown molecule, and double resonance ${ }^{9}$ measurements can verify connectivity between rotational transitions. All of these techniques serve to offer up rotational spectroscopy as an analytical tool for molecular identification.

Besides the dipole moment, the intensity of molecular transitions is also dependent on the shape of the molecule as well as the system temperature. Both of these 
parameters influence the population distribution between energy levels. At higher temperatures, such as room temperature, the population distribution shifts and the transitions of the molecule are seen at higher frequency ranges like the mm-wave regime, while at colder temperatures, such as $2 \mathrm{~K}$, it is best to measure a spectrum in a pulsed jet microwave spectrometer. An analysis of the size can also indicate the best measurement frequency range. In small molecules the rotational energy levels will be further spread apart, making higher frequency ranges ideal for spectral assignment, whereas larger molecules will have a more favorable Boltzmann distribution at lower frequencies. All three of these properties - the dipole moment, the shape and size of the molecule, and the system temperature - should be taken into account when assessing the most appropriate method of analysis.

Another source of information about the molecular conditions of an experiment is the lineshapes of the transitions. The lineshapes can be either homogenous or inhomogeneous and are dependent on the type of broadening effect that is most dominant in the system ${ }^{5}$. The most common types of broadening are Doppler, pressure, and lifetime broadening. Each of these causes the lineshape of the molecular transition to have a distinct line profile, such as a Gaussian profile (Doppler broadening), Lorentzian profile (pressure broadening), or Voigt profile (a mixture of the two). The lineshape of a transition becomes important especially when trying to assign astronomical datasets. Rotational spectroscopy is the most powerful method to identify molecules in the interstellar medium (ISM) ${ }^{10}$. This is because of the unique spectrum that every molecule possesses and the ability to determine the physical conditions from the molecular 
spectrum. The kinematics of a region in a source can be determined from the lineshape profiles and velocities of the particular region. The high level of redundancy of the spectrum as well as the high sensitivity of the method allow for accurate molecular assignments. When coupled with laboratory measurements both in the microwave and mm-wave, the chemistry of astronomical regions can be better understood.

\section{Spectroscopic techniques and applications}

Following World War II, the field of rotational spectroscopy started to become a viable spectroscopic technique. The war saw advances in radar technology which led to

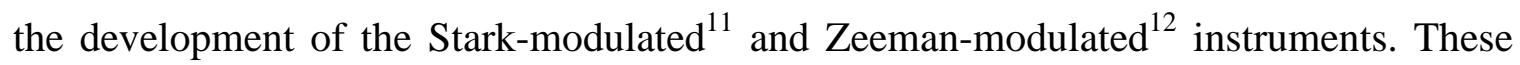
instruments broadcast a continuous wave of radiation through the gas cell and monitor the reduction in amplitude of the wave. Around the same time, Dicke and Romer ${ }^{13}$ (among others) were investigating the most efficient methods to look at the emission spectrum of molecules as opposed to the absorption spectrum. These instruments operated in the radio and microwave regimes, and while the spectrometers were successful at studying a variety of systems ${ }^{14,15}$, many of the instruments operated at room temperature. As stated in Section I, the optimal frequency range to measure the spectra of gas phase molecules at room temperature is in the mm-wave or higher regimes, so these spectrometers were not optimal to measure these systems.

This problem was overcome when the supersonic jet was presented, in which the molecular beam was jet-cooled to a few Kelvin for high-resolution spectroscopy ${ }^{16-18}$. This shifted the Maxwell-Boltzmann distribution of the molecule into the microwave 
regime. An effect of this is that smaller molecules could now be studied with microwave spectroscopy. The next large advance for microwave spectroscopy came when the Flygare group began investigating the use of Fourier transform microwave (FTMW) spectroscopy ${ }^{19-21}$. This eventually led to the Balle-Flygare ${ }^{22}$ design of the Fabry-Perot cavity FTMW spectrometer, which was used in conjunction with the supersonic jet. Instead of monitoring the absorption of a continuous wave by the sample, a pulse of radiation was used to excite the molecular transition. After the radiation pulse left the sample chamber, the molecular emission was collected in the form of its free induction decay (FID). This background free, time domain spectrum was Fourier transformed to yield the frequency spectrum. This method is analogous to FT-NMR techniques ${ }^{23}$. Because it is a background free technique in comparison to absorption spectroscopy, the instrument enjoys higher sensitivity, and the ability to take the Fourier transform of the time domain yields higher frequency resolution than absorption spectroscopy. These two features caused it to gain popularity quickly.

Even with the success of this instrument ${ }^{24-26}$, a major limitation still posed a problem that made the analysis of spectra complicated. The Balle-Flygare FTMW instrument can only operate over small bandwidths $(1 \mathrm{MHz})$. For a molecule that has never been studied before, the calculated transition frequencies are not very accurate for complex systems, depending on the level of theory used. For simple systems and monomers $a b$ initio calculations can determine the rotational frequencies to within $5 \%$ of the experimental values ${ }^{27,28}$; however, for complex systems which are influenced by van der Waals forces the discrepancies can be larger ${ }^{29}$. Therefore, searching for these 
transitions in an instrument like the Balle-Flygare instrument can be lengthy, depending upon the accuracy of the frequency calculations. Improvements, such as the ability to step across frequencies, have been made to the design ${ }^{30}$; however this method of identifying a molecular spectrum is quite time consuming. The ideal situation would be to acquire a broadband spectrum (without a time consuming scan) so that all of the molecular transitions would be present at once for easy identification.

As these techniques were being developed, techniques in the mm-wave regime were also becoming available. These absorption techniques used single frequency sources such as klystrons ${ }^{31-33}$ and Gunn oscillators ${ }^{34}$ to produce high frequency excitation radiation. These instruments were slow and could require a lot of maintenance. In 1997, the FASSST instrument ${ }^{35}$ was developed in order to decrease measurement time needed for a broadband spectrum, as well as increase the sensitivity and improve frequency calibration. Further upgrades to mm-wave instruments have come in the form of computer software ${ }^{36,37}$ and solid-state nonlinear diode circuits $^{36-38}$. While these upgrades have greatly decreased the acquisition time, these instruments are still operating with a continuous wave source. As stated earlier, emission spectroscopy has better sensitivity and thus a mm-wave instrument that acquires emission spectra would be preferable.

The chirped pulse-Fourier transform (CP-FT) technique was first introduced in $2006^{39}$ in the microwave regime. This method allows the user to obtain a broadband spectrum by simultaneously exciting molecules across the bandwidth of the chirped pulse (typically $10 \mathrm{GHz}$ or more). The coherent emission is collected and can be averaged in 
the time domain. It is based on the work done by McGurk et al. ${ }^{40}$ for fast passage excitation. A chirped pulse has the form of:

$$
E(t)=E_{0} \cos \left(\omega_{0} t+\frac{1}{2} \alpha t^{2}\right)
$$

The linear sweep begins at angular frequency $\omega_{0}$ and proceeds with a sweep rate of $\alpha$. The instantaneous frequency of the radiation interacting with the molecular sample is

$$
\omega(t)=\omega_{0}+\alpha t
$$

The chirped pulses can be generated using now available digital electronics such as an arbitrary waveform generator (AWG). The CP-FT technique has been used in the microwave regime from $2-40 \mathrm{GHz}^{41-43}$ and also in the mm-wave regime ${ }^{44-49}$. These instruments offer high sensitivity broadband measurements.

Interest in astronomical detections of molecules started with the first radio astronomy detection of the hydroxyl radical in $1963^{50}$, and continued to have much success both in the microwave and mm-wave regimes. The laboratory measurements were compared with data from single dish telescopes to reveal the molecular composition of dense star forming regions like Sagittarius B2 and the Orion nebula. Many times, the observed species were exotic molecules, such as radicals ${ }^{50}$ and ions ${ }^{51}$, that are not found under terrestrial conditions. Therefore, techniques were developed to aid in the detection and identification of these molecules. Early techniques involved microwave discharge cavities $^{12,52}$ and in the mm-wave regime DC glow discharges ${ }^{53,54}$, while later microwave techniques, such as FTMW and CP-FTMW, were paired with a pulsed discharge nozzle ${ }^{55-60}$. While these techniques have helped aid in the detection of almost 200 interstellar species, new and faster techniques will need to be developed to keep up with 
the future flow of astronomical data. Upgrades to the Karl G. Jansky Very Large Array (VLA) and the completion of the Atacama Large Millimeter Array (ALMA) promise to bring in more data in a year than the all of the currently available radio astronomy data. Techniques must be developed to handle this massive amount of information.

\section{Instrument and astrochemical technique development}

This thesis presents the development of CP-FT techniques as well as single frequency techniques and their applications to radio astronomy. With the ever evolving technology, rotational spectroscopy has had the ability to transform itself several times since the 1940's. The current technology has allowed us to push the CP-FT technique from the microwave regime to the millimeter regime. Chapter 2 will discuss the development of an instrument that uses solid-state light sources, driven by AWGs. The instrument operates between 260-295 GHz, pushing the CP-FT method into the mmwave regime and allowing for direct comparison between this data from this instrument and data from Band 6 and 7 of ALMA. Advantages over current absorption millimeter wave (mm-wave) techniques are investigated, and the performance of the instrument is measured. A comparison of ethyl cyanide measurements from the designed spectrometer and measurements from the FASSST spectrometer ${ }^{35,36}$ is made.

In Chapter 3, the ability to build a low cost version of the spectrometer is assessed as well as the sensitivity and application of such an instrument. The instrument presented in Chapter 2 contains many state-of-the-art electronics, which cause the instrument to be 
quite expensive. In order to take advantage of mm-wave technology but at a lower cost, while still performing time domain, Fourier transform spectroscopy, monolithic microwave integrated circuits (MMIC) are used to replace the AWG. This reduces the measurement bandwidth as a transform limited pulse is used. Even though this is the case, the instrument is able to completely characterize carbonyl sulfide (OCS) through a series of measurements, opening it up to the possibilities of analytical chemistry applications.

The study presented in Chapter 4 explores the idea of implementing the low cost instrumentation in the microwave regime both for analytical uses as well as for astrochemistry. The Fourier transform microwave (FTMW) spectrometer operates with no moving parts and is quickly reprogrammable. Like its mm-wave counterpart, this instrument possesses the ability to be able to characterize the dipole moment, spectrum, and eventually identity of either a known or unknown molecules. When paired with the automation abilities of the spectrometer, data can be collected and analyzed quickly with the click of a button from a programmed graphical user interface. Measurements of ammonia demonstrate these abilities as well as the sensitivity of the instrument. Beyond molecular characterization, this instrument's secondary role is as an aid for the public to understand the field of astrochemistry in a traveling museum exhibit. The spectrum of the molecule is compared to a pre-existing astronomical survey to show how astronomical detections are made.

In Chapter 5 the instrument developed in Chapter 2 is discussed with modifications for astrochemical purposes. Many radio astronomy facilities have recently 
come online that produce large amounts of spatial and spectral data. In order to keep up with the amount of data produced by these observatories, the bottleneck that has developed in getting laboratory data out for new molecules needs to be tackled. One way to do this is by developing a fast and sensitive laboratory method such as the segmented CP-FT mm-wave discharge technique. A pulsed discharge nozzle ${ }^{56}$ is coupled to the chamber, so that products from the discharge of acetonitrile can monitored, using the segmented CP-FT mm-wave approach. The effectiveness of the technique is determined by comparing the mm-wave results to results from the microwave regime, as it has already been demonstrated that discharge CP-FTMW techniques are successful at producing interstellar molecules ${ }^{60-62}$.

The second focus of this thesis is the development of tools to help with the analysis of astrochemical datasets. In Chapter 6, a proposed reaction mechanism for the formation of E-cyanomethanimine in Sagittarius B2 (Sgr B2) is investigated. Ecyanomethanimine $^{61}$ was first discovered by the broadband reaction screening method ${ }^{59}$ that was developed with the CP-FTMW spectrometer in this lab, but no viable reaction mechanism has been able to explain its formation in the ISM. The addition of the cyanide radical to methanimine to yield the E-cyanomethanimine has been proposed based off of work done by Balucani and co-workers ${ }^{63}$ and is investigated via the spatial distribution screening method. Data from the interferometer, the VLA, were acquired and correlations between the spatial distributions of the reactants and the product are studied to try and validate the proposed reaction mechanism. 
Finally Chapter 7 will look more closely at the concept of the spatial distribution screening method. As the larger datasets become available, faster and more efficient tools to analyze them will be necessary. Thus the spatial distribution screening method has been developed. The datasets that will be produced by ALMA contain not only spectral information but also spatial information to give a "3D" view of the observed source. To comb through the entire dataset would take months if not years, and it could be quite difficult to locate less abundant molecules in the sea of lines that exists for warmer regions, such as hot cores. With the spatial distribution method, a reaction to form the molecule of interest is proposed, and using the location of the reactants, the product can be searched for in a systematic manner. The method is tested by looking for both HSCN and E-cyanomethanimine in the Orion Kleinmann-Low nebula (Orion KL). Through the development of laboratory techniques and methods to analyze astronomical data, a large effort can be made to not only know what the chemical composition of a source is but also the chemistry that is occurring to generate the composition. 


\section{Chapter 1 References}

(1) Townes, C. H.; Schawlow, A. L. Microwave Spectroscopy; 1st ed.; Dover Publications, Inc.: Mineola, New York, 1975.

(2) Gordy, W.; Cook, R. L. Microwave Molecular Spectra; 3rd ed.; John Wiley and Sons: New York, 1984.

(3) Brewer, R. G.; Shoemaker, R. L. Phys. Rev. Lett. 1971, 27, 631-634.

(4) Hocker, G. B.; Tang, C. L. Phys. Rev. 1969, 184, 356-359.

(5) Bernath, P. F. Spectra of Atoms and Molecules; Second.; Oxford University Press: New York, 2005.

(6) Hahn, E. L. Phys. Rev. 1950, 80, 580-594.

(7) McGurk, J. C.; Hofmann, R. T.; Flygare, W. H. J. Chem. Phys. 1974, 60, 29222928.

(8) Mäder, H.; Ekkers, J.; Hoke, W.; Flygare, W. H. J. Chem. Phys. 1975, 62, 43804387.

(9) Wodarczyk, F. J.; Wilson, E. B. J. Mol. Spectrosc. 1971, 37, 445-463.

(10) Herbst, E.; van Dishoeck, E. F. Annu. Rev. Astron. Astrophys. 2009, 47, 427-480.

(11) Jr, K. B. M.; Hughes, R. H.; Jr, E. B. W. Rev. Sci. Instrum. 1949, 20, 821-826.

(12) Ehrenstein, G.; Townes, C. H.; Stevenson, M. J. Phys. Rev. Lett. 1959, 3, 40-41.

(13) Dicke, R. H.; Romer, R. H. Rev. Sci. Instrum. 1955, 26, 915-928.

(14) Scroggin, D. G.; Riveros, J. M.; Wilson, E. B. J. Chem. Phys. 1974, 60, 13761385.

(15) Ford, R. G.; Beaudet, R. A. J. Chem. Phys. 1971, 55, 3110-3113. 
(16) Anderson, J. B.; Andres, R. P.; Fenn, J. B. In Advances in Chemical Physics; Ross, J., Ed.; John Wiley \& Sons, Inc., 1966; pp. 275-317.

(17) Dyke, T. R.; Howard, B. J.; Klemperer, W. J. Chem. Phys. 1972, 56, 2442-2454.

(18) Smalley, R. E.; Wharton, L.; Levy, D. H. Acc. Chem. Res. 1977, 10, 139-145.

(19) Ekkers, J.; Flygare, W. H. Rev. Sci. Instrum. 1976, 47, 448-454.

(20) Campbell, E. J.; Buxton, L. W.; Balle, T. J.; Flygare, W. H. J. Chem. Phys. 1981, 74, 813-828.

(21) Campbell, E. J.; Buxton, L. W.; Balle, T. J.; Keenan, M. R.; Flygare, W. H. J. Chem. Phys. 1981, 74, 829-840.

(22) Balle, T. J.; Flygare, W. H. Rev. Sci. Instrum. 1981, 52, 33-45.

(23) Gunther, H. NMR Spectroscopy; John Wiley and Sons: New York, 1995.

(24) Serafin, M. M.; Peebles, S. A. J. Phys. Chem. A 2008, 112, 12616-12621.

(25) Cooke, S. A.; Gerry, M. C. L. J. Mol. Spectrosc. 2005, 234, 195-203.

(26) Sanz, M. E.; Lesarri, A.; Peña, M. I.; Vaquero, V.; Cortijo, V.; López, J. C.; Alonso, J. L. J. Am. Chem. Soc. 2006, 128, 3812-3817.

(27) Maroulis, G.; Simos, T. Trends and Perspectives in Modern Computational Science; CRC Press, 2006.

(28) Puzzarini, C.; Heckert, M.; Gauss, J. J. Chem. Phys. 2008, 128, 194108.

(29) Seifert, N. A.; Steber, A. L.; Neill, J. L.; Pérez, C.; Zaleski, D. P.; Pate, B. H.; Lesarri, A. Phys. Chem. Chem. Phys. PCCP 2013, 15, 11468-11477.

(30) Grabow, J.-U.; Stahl, W.; Dreizler, H. Rev. Sci. Instrum. 1996, 67, 4072-4084.

(31) King, W. C.; Gordy, W. Phys. Rev. 1953, 90, 319-320. 
(32) King, W. C.; Gordy, W. Phys. Rev. 1954, 93, 407-412.

(33) Burrus, C. A.; Gordy, W. Phys. Rev. 1956, 101, 599-602.

(34) McCarthy, M. C.; Gottlieb, C. A.; Cooksy, A. L.; Thaddeus, P. J. Chem. Phys. 1995, 103, 7779-7787.

(35) Petkie, D. T.; Goyette, T. M.; Bettens, R. P. A.; Belov, S. P.; Albert, S.; Helminger, P.; Lucia, F. C. D. Rev. Sci. Instrum. 1997, 68, 1675-1683.

(36) Medvedev, I. R.; Neese, C. F.; Plummer, G. M.; De Lucia, F. C. Opt. Lett. 2010, 35, 1533-1535.

(37) Drouin, B. J.; Maiwald, F. W.; Pearson, J. C. Rev. Sci. Instrum. 2005, 76, 093113.

(38) Crowe, T. W.; Bishop, W. L.; Porterfield, D. W.; Hesler, J. L.; Weikle, R. M. IEEE J. Solid-State Circuits 2005, 40, 2104-2110.

(39) Brown, G. G.; Dian, B. C.; Douglass, K. O.; Geyer, S. M.; Pate, B. H. J. Mol. Spectrosc. 2006, 238, 200-212.

(40) McGurk, J. C.; Mäder, H.; Hofmann, R. T.; Schmalz, T. G.; Flygare, W. H. J. Chem. Phys. 1974, 61, 3759-3767.

(41) Pérez, C.; Lobsiger, S.; Seifert, N. A.; Zaleski, D. P.; Temelso, B.; Shields, G. C.; Kisiel, Z.; Pate, B. H. Chem. Phys. Lett. 2013, 571, 1-15.

(42) Brown, G. G.; Dian, B. C.; Douglass, K. O.; Geyer, S. M.; Shipman, S. T.; Pate, B. H. Rev. Sci. Instrum. 2008, 79, 053103.

(43) Zaleski, D. P.; Neill, J. L.; Muckle, M. T.; Seifert, N. A.; Brandon Carroll, P.; Widicus Weaver, S. L.; Pate, B. H. J. Mol. Spectrosc. 2012, 280, 68-76. 
(44) Neill, J. L.; Harris, B. J.; Steber, A. L.; Douglass, K. O.; Plusquellic, D. F.; Pate, B. H. Opt. Express 2013, 21, 19743-19749.

(45) Park, G. B.; Steeves, A. H.; Kuyanov-Prozument, K.; Neill, J. L.; Field, R. W. J. Chem. Phys. 2011, 135, 024202.

(46) Oldham, J. M.; Abeysekera, C.; Joalland, B.; Zack, L. N.; Prozument, K.; Sims, I. R.; Park, G. B.; Field, R. W.; Suits, A. G. J. Chem. Phys. 2014, 141, 154202.

(47) Gerecht, E.; Douglass, K. O.; Plusquellic, D. F. Opt. Express 2011, 19, 8973-8984.

(48) Prozument, K.; Colombo, A. P.; Zhou, Y.; Park, G. B.; Petrović, V. S.; Coy, S. L.; Field, R. W. Phys. Rev. Lett. 2011, 107, 143001.

(49) Harris, B. J. A Chirped Pulse Fourier Transform Millimeter Wave Spectrometer for Room Temperature, Gas Mixture Analysis. Doctor of Philosophy, University of Virginia, 2014.

(50) Weinreb, S.; Barrett, A. H.; Meeks, M. L.; Henry, J. C. Nature 1963, 200, 829831.

(51) McCarthy, M. C.; Gottlieb, C. A.; Gupta, H.; Thaddeus, P. Astrophys. J. Lett. 2006, 652, L141.

(52) Fehsenfeld, F. C.; Evenson, K. M.; Broida, H. P. Rev. Sci. Instrum. 1965, 36, $294-$ 298.

(53) Pearson, J. C.; Gottlieb, C. A.; Woodward, D. R.; Thaddeus, P. Astron. Astrophys. 1988, 189, L13-L15.

(54) Thaddeus, P.; McCarthy, M. C. Spectrochim. Acta. A. Mol. Biomol. Spectrosc. 2001, 57, 757-774. 
(55) McCarthy, M. C.; Travers, M. J.; Kovács, A.; Gottlieb, C. A.; Thaddeus, P. Astrophys. J. Suppl. Ser. 1997, 113, 105.

(56) McCarthy, M. C.; Chen, W.; Travers, M. J.; Thaddeus, P. Astrophys. J. Suppl. Ser. 2000, 129, 611 .

(57) Iida, M.; Ohshima, Y.; Endo, Y. Astrophys. J. Lett. 1991, 371, L45.

(58) Sanz, M. E.; McCarthy, M. C.; Thaddeus, P. J. Chem. Phys. 2003, 119, 1171511727.

(59) Zaleski, D. P.; Stephens, S. L.; Walker, N. R. Phys. Chem. Chem. Phys. 2014.

(60) Neill, J. L.; Muckle, M. T.; Zaleski, D. P.; Steber, A. L.; Pate, B. H.; Lattanzi, V.; Spezzano, S.; McCarthy, M. C.; Remijan, A. J. Astrophys. J. 2012, 755, 153.

(61) Zaleski, D. P.; Seifert, N. A.; Steber, A. L.; Muckle, M. T.; Loomis, R. A.; Corby, J. F.; Oscar Martinez, J.; Crabtree, K. N.; Jewell, P. R.; Hollis, J. M.; Lovas, F. J.; Vasquez, D.; Nyiramahirwe, J.; Sciortino, N.; Johnson, K.; McCarthy, M. C.; Remijan, A. J.; Pate, B. H. Astrophys. J. Lett. 2013, 765, L10.

(62) Loomis, R. A.; Zaleski, D. P.; Steber, A. L.; Neill, J. L.; Muckle, M. T.; Harris, B. J.; Hollis, J. M.; Jewell, P. R.; Lattanzi, V.; Lovas, F. J.; Oscar Martinez, J.; McCarthy, M. C.; Remijan, A. J.; Pate, B. H.; Corby, J. F. Astrophys. J. Lett. 2013, 765, L9.

(63) Balucani, N.; Asvany, O.; Huang, L. C. L.; Lee, Y. T.; Kaiser, R. I.; Osamura, Y.; Bettinger, H. F. Astrophys. J. 2000, 545, 892-906. 


\section{Chapter 2}

\section{An Arbitrary Waveform Generator Based Chirped Pulse Fourier Transform Spectrometer Operating from $260-295 \mathrm{GHz}^{1}$}

\section{Introduction}

Chirped-pulse Fourier transform (CP-FT) microwave spectroscopy provides broadband spectral coverage on each single spectrum acquisition ${ }^{2-4}$. The potential of CPFT microwave spectroscopy was recognized early in the development of the field of Fourier transform microwave spectroscopy through experiments that used the molecular Stark effect to achieve rapid frequency sweeps through resonance ${ }^{5}$. The essential advantage of CP-FT spectroscopy for high-resolution spectroscopy comes from the fact that the relaxation rate of the coherent molecular free induction decay (FID), which defines the line width for each spectral feature, is much longer than the reciprocal of the spectrum bandwidth being measured. In the case where the spectrum is excited by a transform limited pulse, the pulse duration is set by the full measurement bandwidth. In most cases for rotational spectroscopy, the peak power of the excitation source is insufficient to achieve optimal excitation over the full operating bandwidth when a short, transform-limited pulse is used. The chirped-pulse, with linear frequency sweep, "stretches" the excitation pulse to match the time scale of the molecular relaxation, roughly the reciprocal of the individual transition line width, which can be orders-ofmagnitude longer than the time scale set by the total measurement bandwidth. The chirped-pulse delivers the maximum total pulse energy for each measurement to achieve 
the highest full-spectrum measurement sensitivity possible with the fixed peak power source.

Although the concept for CP-FT spectroscopy was known from early work, the development of spectrometers based on this optimal excitation scheme required the development of high-speed digital electronics for both pulse generation and broadband detection of the FID. Arbitrary waveform generators (AWGs) are needed to achieve the sweep rates required for CP-FT spectroscopy where pulses with bandwidths of 10-100 GHz and pulse durations of $10 \mathrm{~ns}-1 \mu \mathrm{s}$ are required. These sweep rates are not attainable with conventional microwave sources. In addition, the sensitivity of CP-FT techniques comes from the ability to average the coherent FID signal in the time domain over many spectrum acquisitions - often exceeding 1 million FID averages ${ }^{6}$. Phasereproducible excitation pulses are required, and AWG-based sources readily provide this phase control. Following chirped-pulse excitation, the FID is digitized and the frequency domain spectrum is produced by fast Fourier transform of the time-domain response. The bandwidth of each single observation is limited by the properties of the digitizer (both the sample rate and the specifications of the hardware bandwidth limits on the sample input port). By 2005-2006, digital electronics advanced to the point where CP-FT spectroscopy over bandwidths of $10 \mathrm{GHz}$ and higher became possible ${ }^{2}$. Since that time, digital electronics have continued to advance on a Moore's Law -like development curve where the digitizer bandwidth doubles about every 36 months ${ }^{7}$. The current state-of-theart is the Agilent Infiniium 90000 Q-Series $160 \mathrm{GS} / \mathrm{s}$ digitizer with the ability to acquire signals with $63 \mathrm{GHz}$ of bandwidth. 
Chirped-pulse Fourier transform spectroscopy is best suited for high-resolution spectroscopy applications where the excitation power is significantly higher than the power required to saturate the transition. Under these conditions, CP-FT spectroscopy provides a way to efficiently use the full power of the light source and reduce the total measurement time. Solid-state mm-wave-to- $\mathrm{THz}$ sources generate output powers well above that required for saturation ${ }^{8}$ and have reasonably broadband spectral coverage (10-20\% of the center frequency), making the extension of CP-FT spectroscopy to this frequency region advantageous for several reasons. First, the chirped excitation pulse is ideally suited to the active frequency multiplier chains used to generate mm-wave power because these devices increase the bandwidth of the chirped pulse by the frequency multiplication factor of the chain without changing the pulse duration. Therefore, relatively low AWG bandwidth is required to create mm-wave pulses that cover the full operating range of the mm-wave source. Secondly, CP-FT spectrometers use high accuracy frequency standards (most commonly $10 \mathrm{MHz}$ rubidium disciplined crystal oscillators) to synchronize the AWG and digitizer. As a result, frequency calibration of the spectrometer is achieved directly in the fast Fourier transform analysis of the digitized FID. For broadband CP-FTmm-wave spectrometers, the excitation for molecules is in the weak pulse limit (although this condition is not met for Rydberg atoms and molecules ${ }^{9}$ ). Compared to traditional frequency-tuned absorption spectroscopy, this removes the need to optimize the source power for each individual molecule to take into account power saturation effects ${ }^{8}$. 
Another key advantage is that a broadband rotational spectrum is obtained in the relaxation time of a single transition, which is on the order of $1 \mu$ s for mm-wave spectroscopy at typical pressures and Doppler dephasing times. In traditional frequencyswept absorption spectroscopy the frequency scan rate is limited by the molecular relaxation rate, which fixes the minimum amount of time for scanning a single spectral line $e^{8,10,11}$. The time resolution of CP-FT spectroscopy makes it possible to synchronize the broadband spectrum detection to transient events that might be initiated by laser excitation or pulsed discharge sources for producing high-energy species. Finally, the method is a coherent spectroscopy and has possibilities for developing selectiveexcitation $^{4,12}$ and fully two-dimensional (2D) double-resonance techniques for the analysis of complex spectra ${ }^{13}$.

In 2011, two groups extended CP-FT techniques to mm-wave frequencies using solid-state devices for the excitation source. The Field group at MIT developed a spectrometer in the $70-102 \mathrm{GHz}$ frequency region for atomic ${ }^{9}$ and molecular spectroscopy ${ }^{14}$. Gerecht, Douglass, and Plusquellic at NIST demonstrated a broadband (10 $\mathrm{GHz}$ bandwidth) spectrometer working at frequencies above $500 \mathrm{GHz}$ that is applicable to studies of room-temperature gas samples ${ }^{15}$. The design of a CP-FT spectrometer operating in the $260-295 \mathrm{GHz}$ frequency band is presented in this chapter. The spectrometer uses solid-state active frequency multipliers with peak power of $10-30 \mathrm{~mW}$ across the band as the excitation source. The molecular rotational spectrum across the full bandwidth of the light source can be detected in a single spectrum acquisition. We present a simplified experimental design for this spectrometer that uses a 
single-channel AWG to create both the chirped excitation pulse and the local oscillator frequency for the spectrum downconversion.

\section{Experimental}

Schematic diagrams of the two implementations of broadband CP-FTmm-wave spectroscopy we have tested are shown in Fig. 2.1. The designs of the excitation source, receiver, and sample chamber are described below.

a) Chirped-pulse excitation source

The excitation source is a Virginia Diodes, Inc. (VDI) active multiplier chain (AMC) that has an overall frequency multiplication factor of 24. An input frequency range of $10.8-12.3 \mathrm{GHz}$ (just $1.5 \mathrm{GHz}$ of bandwidth) to the AMC linearly addresses the final frequency output range of $260-295 \mathrm{GHz}$. The output power is $10-30 \mathrm{~mW}$ across the 260-295 GHz frequency range. Chirped-pulse generation uses a high-speed (12 GS/s) arbitrary waveform generator (AWG, Tektronix 7122B). The AWG uses an external $12 \mathrm{GHz}$ phase-locked dielectric oscillator (PDRO) for its clock. Waveforms are created on the AWG in the frequency range of $2.0-3.5 \mathrm{GHz}$ and are frequency upconverted to the required 10.8-12.3 GHz AMC input range using a mixer and a bandpass filtered single frequency PDRO operating at $8.8 \mathrm{GHz}$. Both PDRO clock sources derive their frequencies through multiplication of an input $10 \mathrm{MHz}$ rubidium frequency standard (SRS FS725). The upper sideband produced in the mixing of the AWG and the PDRO is 

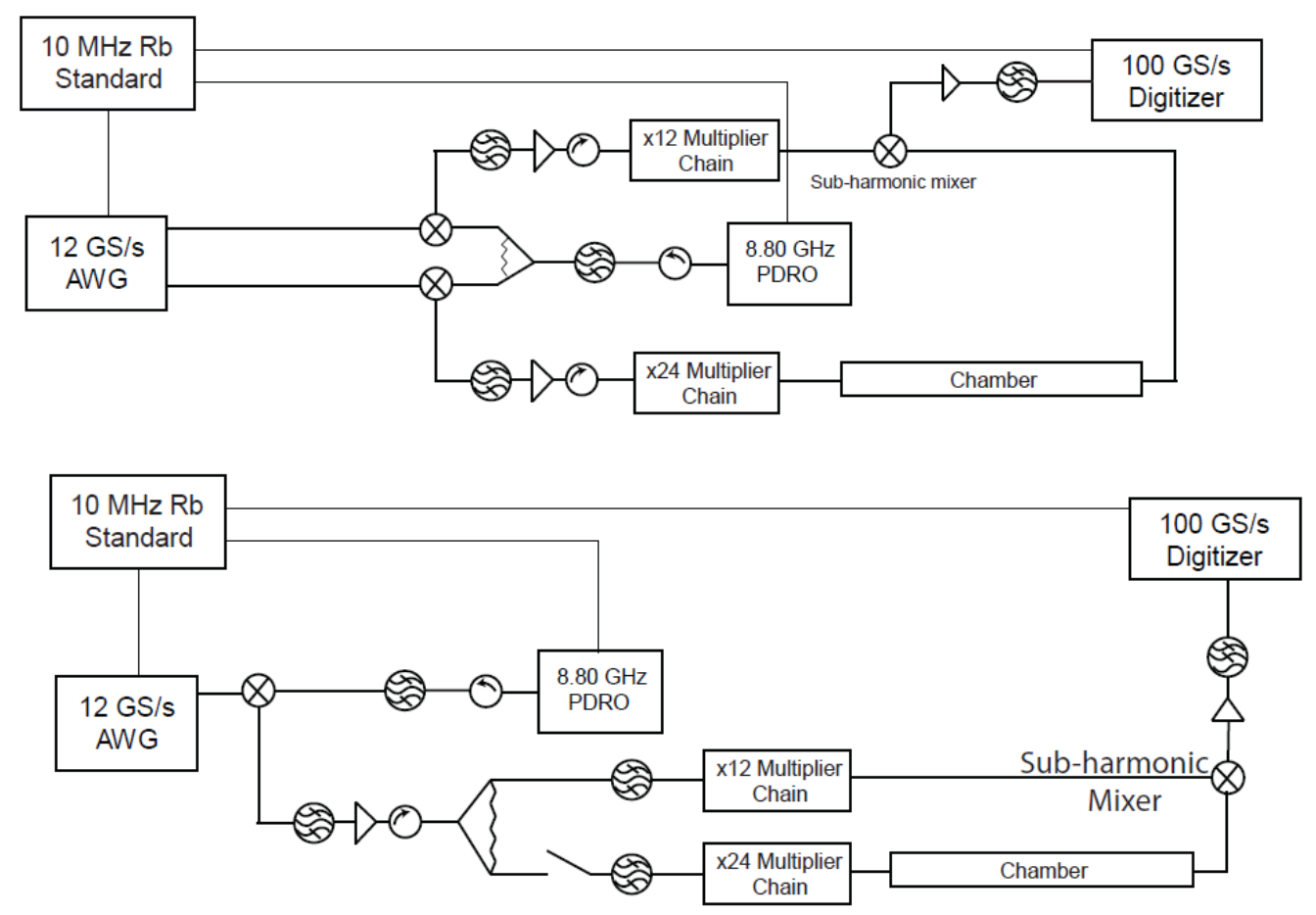

Figure 2.1. Schematics of the dual channel (top) and single channel (bottom) circuits. In the dual channel circuit, the chirped pulse and the LO are generated from separate AWG channels. The $8.8 \mathrm{GHz}$ PDRO is power divided in order to upconvert each waveform for input to their respective multiplier chains. In the single channel circuit, both the chirped pulse and the LO originate from the same AWG channel. The time separated waveforms are separated for input to the detector and AMC source by a single throw switch. 
selected by a bandpass filter and amplified to about $18 \mathrm{dBm}$. Additional attenuation ensures the RF input power complies with safe operation of the AMC.

The output power of the AMC that can be used in the experiment is limited by the power handling capabilities of the mixer in the receiver system. We are currently setting the power of the excitation pulse observed at the receiver to $1 \mathrm{~mW}$. However, this specification from VDI is for damage by continuous power input to the receiver, and it is possible that the input power can be increased by a factor of 10 (thereby increasing the single acquisition signal levels by the square root of 10) in the low-duty cycle CP-FT measurements. We have not verified this performance, however.

The chirped-pulse input to the AMC needs to have low spurious content to produce clean excitation pulses at $260-290 \mathrm{GHz}$. We have been particularly careful to exclude harmonic content from the AWG by operating it in the $2.0-3.5 \mathrm{GHz}$ region. Harmonics $(4-7 \mathrm{GHz})$ are filtered out by the bandpass filter after the mixer. In the absence of a driving pulse to the AMC, there is no detectable noise power output from the AMC source that could be a noise source during FID collection.

\section{b) Receiver}

The molecular free induction decay is detected using a room-temperature subharmonic mixer (SHM). This mixer operates over the full WR 4.3 waveguide band from 220-325 GHz with a conversion loss of $9 \mathrm{~dB}$. The local oscillator for the $\mathrm{SHM}$ is produced in an AMC with overall frequency multiplication factor of 12 (a SHM takes an input at half the final local oscillator (LO) frequency). The IF bandwidth of the SHM is $40 \mathrm{GHz}$, permitting detection of the full $\mathrm{mm}$-wave chirped-pulse excitation range. On the 
IF port of the SHM, a DC block is used to reduce the risk of electrostatic discharge damage of the unit. The IF signal is amplified by $45 \mathrm{~dB}$ using a low-noise broadband amplifier (noise figure $4.0 \mathrm{~dB}$, Miteq J32-0200400-57-5P) and transmitted to the digitizer on a cable. This signal is digitized by a $100 \mathrm{GS} / \mathrm{s}$ digital oscilloscope (Tektronix DPO 73304D), which has an input bandwidth specification of $33 \mathrm{GHz}$; however, we have observed the ability to detect signals out to $40 \mathrm{GHz}$ with no loss in sensitivity ${ }^{16}$. The digitizer is locked to the same $10 \mathrm{MHz}$ reference signal as the PDROs used in pulse upconversion, and digitization is initiated by a marker channel trigger from the AWG.

\section{c) Single- and dual-channel spectrometer designs}

Two instrument designs have been tested. In the first design the LO frequency generation occurs on one of the AWG channels, while in the second, the LO is generated on the same channel as the chirped-pulse waveform. In the dual AWG channel implementation, the local oscillator is produced on its own AWG channel as a single frequency sine wave at $1900 \mathrm{MHz}$ that is upconverted to $10.7 \mathrm{GHz}$ using the $8.8 \mathrm{GHz}$ PDRO. In this case, the PDRO output is power divided and used in two separate mixers: one for the chirped pulse and one for the LO. To create a LO with high spectral purity, the $10.7 \mathrm{GHz}$ mixer output is filtered by a narrow bandpass filter $(200 \mathrm{MHz})$ to remove any frequencies generated by AWG spurious signals. Spectral purity of the LO is especially important for CP-FT measurements where the electric field of the molecular FID, not its power, is linear in the number density ${ }^{3,5}$. Spurious signals at the LO input will create images of the molecular spectrum that are proportional to the electric field of 
the spurious LO frequency. In benchmark tests, the AWG-generated LO has the same performance as an LO generated using the spectrally pure output of a frequency synthesizer at $1900 \mathrm{MHz}$. In the dual channel implementation, the LO frequency is output prior to the chirped-pulse creation and remains active through the duration of the FID collection. One benefit of this setup is that the chirped-pulse that is transmitted through the sample chamber can be monitored directly (with removal of the high-gain preamplifier) to assess the quality of the chirped excitation pulse.

The single AWG channel spectrometer design reduces the number of components required for the spectrometer (with an associated cost reduction). In this case, the AWG generates a single waveform that includes the chirped excitation pulse followed by an immediate switch to the single frequency $(1900 \mathrm{MHz}) \mathrm{LO}$ signal. The output of the frequency upconversion step is power divided into two signal lines. A TTL-driven microwave PIN-diode switch is used on the signal line that generates the chirpedexcitation pulse to pass only the chirped pulse to the x 24 AMC. The TTL pulse controlling the switch is generated on a marker channel of the AWG. The narrow bandpass filter on the signal line for the LO passes only the spectrally pure $10.7 \mathrm{GHz}$ signal to the AMC that drives the SHM receiver.

The single AWG channel instrument design is motivated by the fact that excitation and detection are time separated events in CP-FT spectroscopy. Therefore, the receiver does not need to be in its proper operating state until the end of the chirped excitation pulse. In addition, the chirped-pulse must pass through the sample cell, which adds a time delay between when the pulse and the LO reach the receiver. This makes it 
possible for the LO pulse to turn on and have all transient responses dissipate before the receiver starts to downconvert the molecular free induction decay. In practice, we find that the main delay, or dead time, in the experiment is the recovery of the high gain, low noise IF amplifier from saturation once the chirped excitation pulse ends. (There is no way to protect the receiver from the excitation pulse for mm-wave measurements due to the lack of fast, broadband switches in this frequency region, and placing a switch between the SHM and IF amplifier adds the switch conversion loss to the overall receiver noise figure while producing transients of its own.) This dead time is about $200 \mathrm{~ns}$ in our system and appears to be longer than any transient settling time of the SHM. We have seen no difference in receiver performance for single versus dual channel operation.

\section{d) Sample chamber}

The sample chamber is a rectangular stainless steel box with dimensions 20 x $25 \mathrm{x}$ $90 \mathrm{~cm}$ and achieves vacuum through a Varian VHS-6 diffusion pump. The pressure in the chamber is monitored using a 50 mTorr range Baratron capacitance manometer. Typical sample pressures for the experiments are $0.5-5$ mTorr of the pure sample. The liquid samples can be introduced into the chamber by injection of the liquid $(<1 \mu \mathrm{L})$ through a rubber septum. The mm-wave beam is coupled into and out of the chamber using $500 \mathrm{~mm}$ and $300 \mathrm{~mm}$ focal length Teflon lenses, respectively (Thor Labs). The mm-wave excitation pulse makes four passes through the chamber for a total path length of about $4 \mathrm{~m}$. An additional $500 \mathrm{~mm}$ Teflon lens and spherical mirrors are used in this beam path to refocus the beam. 


\section{Results}

The performance of the CP-FT mm-wave spectrometer is benchmarked using ethyl cyanide. This molecule is chosen because the spectra can be compared to measurements using the De Lucia FASSST technique ${ }^{17,18}$ that have recently been made available as part of a temperature-dependent spectroscopy study ${ }^{19}$ of this astrochemically important molecule. The broadband spectrum of ethyl cyanide at room-temperature is shown in Fig. 2.2. The sample pressure is 5 mTorr which is optimal for the signal-tonoise ratio in the CP-FT spectrometer. Each measurement cycle uses a 250 ns duration chirped pulse with a linear frequency sweep from 260-295 GHz. The FID is collected for $1.75 \mu$ s following the chirped pulse for a total spectrum measurement time of $2 \mu \mathrm{s}$. The FID decay includes contributions from both the Doppler dephasing (represented as $\mathrm{T}_{2}$ ), with a $\mathrm{T}_{2}$ of about $700 \mathrm{~ns}$, and collisional self-broadening.

The ethyl cyanide spectrum was measured using the fast frame capability of the oscilloscope. In this mode, the scope acquires a series of back-to-back spectra with no time delay between successive spectrum acquisitions. The number of spectrum "frames" that can be collected in a single real-time event is determined by the memory depth of the fast digitizer memory. An average of these "frames" is calculated and this single, "average" frame is passed to the standard memory of the oscilloscope for additional timedomain averaging. This signal processing mode allowed us to achieve effective signal averaging rates of $670 \mathrm{~Hz}$, a significant improvement over the $90 \mathrm{~Hz}$ averaging rate we observed in the "standard" oscilloscope averaging mode. 


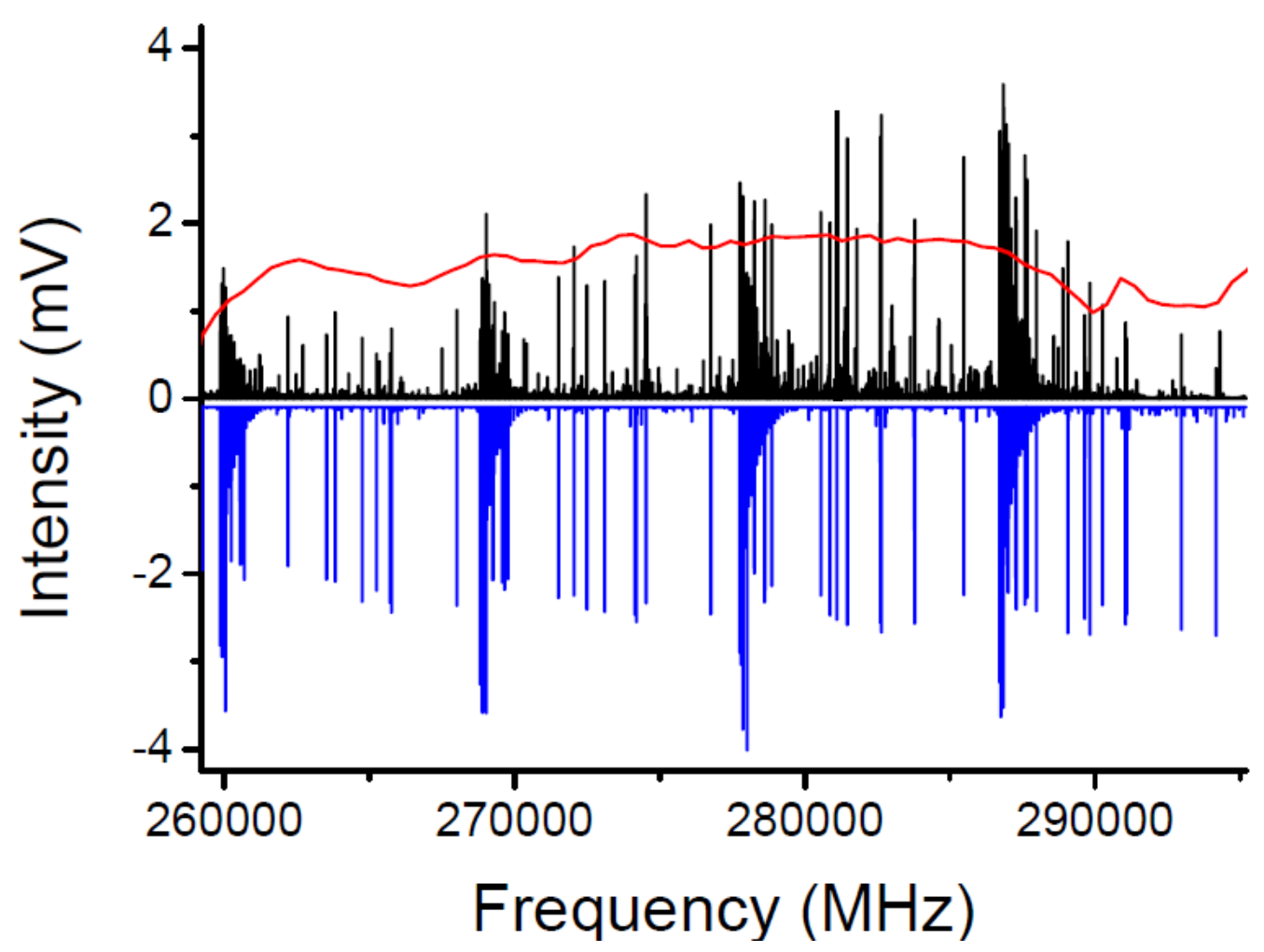

Figure 2.2. A 400 average spectrum of ethyl cyanide taken at 5mTorr from 259.2-295.2 GHz with the 100GS/s Tektronix oscilloscope. Overlaid in red is the power response of the excitation source VDI multiplier chain. The JPL simulation ${ }^{20}$ is plotted in blue. 
It is also possible to use the fast frame capabilities without calculating the average frame. In this case, a back-to-back sequence of broadband spectra can be acquired, and the full set of frames can be transferred to the standard memory for signal averaging. Because a much longer data record is transferred, this mode will not be able to achieve as high a repetition rate. However, it provides a way to follow the evolution of the mmwave spectrum following a transient event initiated, for example, by laser excitation with $2 \mu \mathrm{s}$ or less time resolution.

We have used the reference FASSST spectra of ethyl cyanide to assess the accuracy of the relative intensities in the CP-FT spectrum. A comparison between the 10,000 average CP-FT spectrum, taken with the single channel circuit, and the FASST spectrum in a region where these two measurements overlap is shown in Fig. 2.3. The CP-FT relative intensities are within 10\% agreement with the FASSST spectra and are sufficient for using relative intensity information to guide spectral assignments. As was described above, we have tested spectrometer designs that use both dual and single AWG channels to generate the excitation pulse and LO. In order to demonstrate the equivalence of the two methods, a comparison of ethyl cyanide spectra measured using the two approaches is shown in Fig. 2.4.

An important consideration for the performance of broadband CP-FTmm-wave spectroscopy is the line width and line shape of the spectrum. The line shape properties of the CP-FT measurement are compared to the absorption spectroscopy FASSST measurements in Fig. 2.5. In this comparison, the sample pressure is $0.5 \mathrm{mTorr}$ for both measurements and the line shape in the FASSST spectrum is dominated by the Doppler 


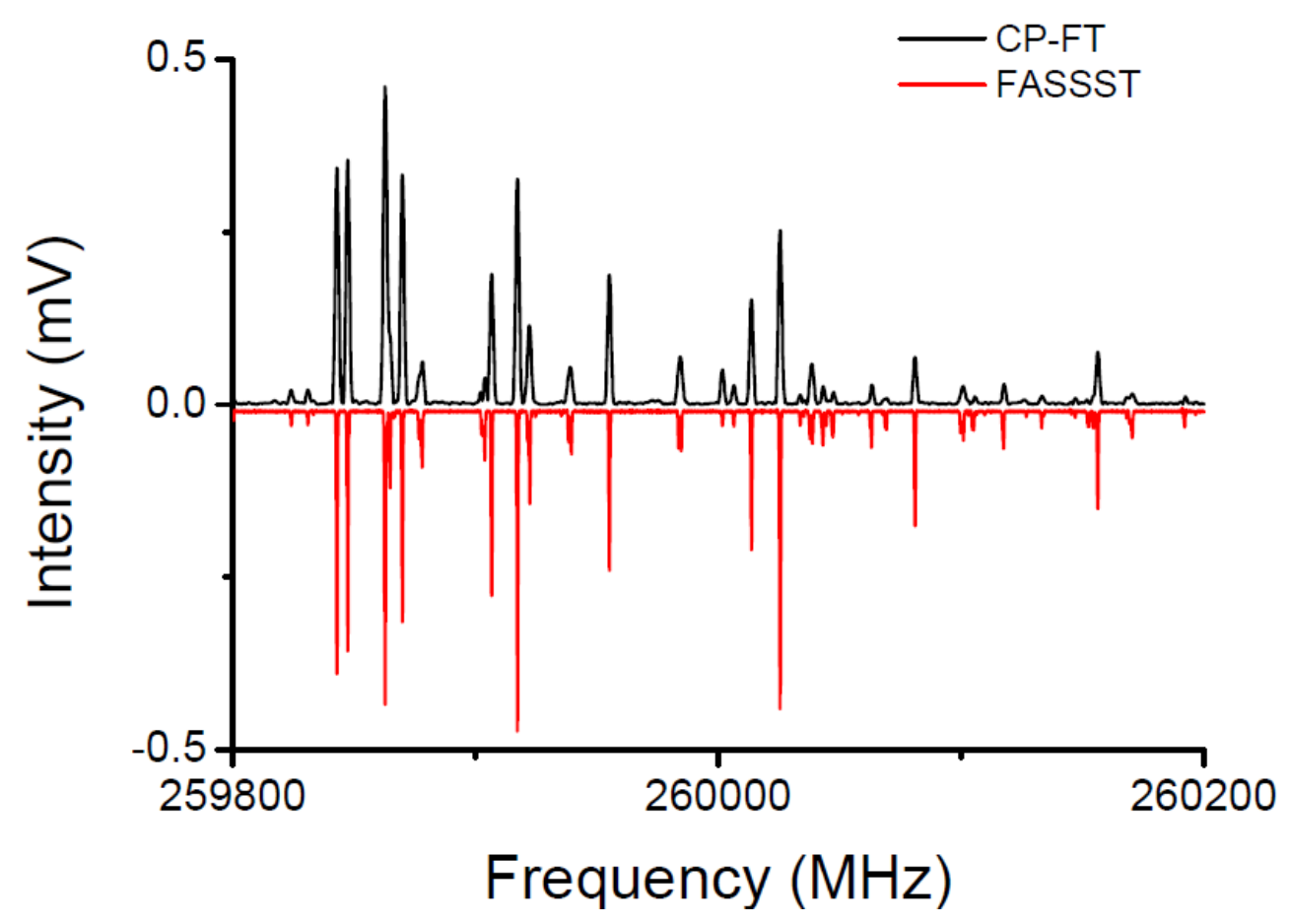

Figure 2.3. A 10,000 average spectrum of ethyl cyanide from $259.8-260.2 \mathrm{GHz}$ matched with the data obtained from the FASSST spectrometer at $298 \mathrm{~K}$. This spectrum was measured with the single channel setup (see Figure 2.1) at 0.5 mTorr of pressure. 


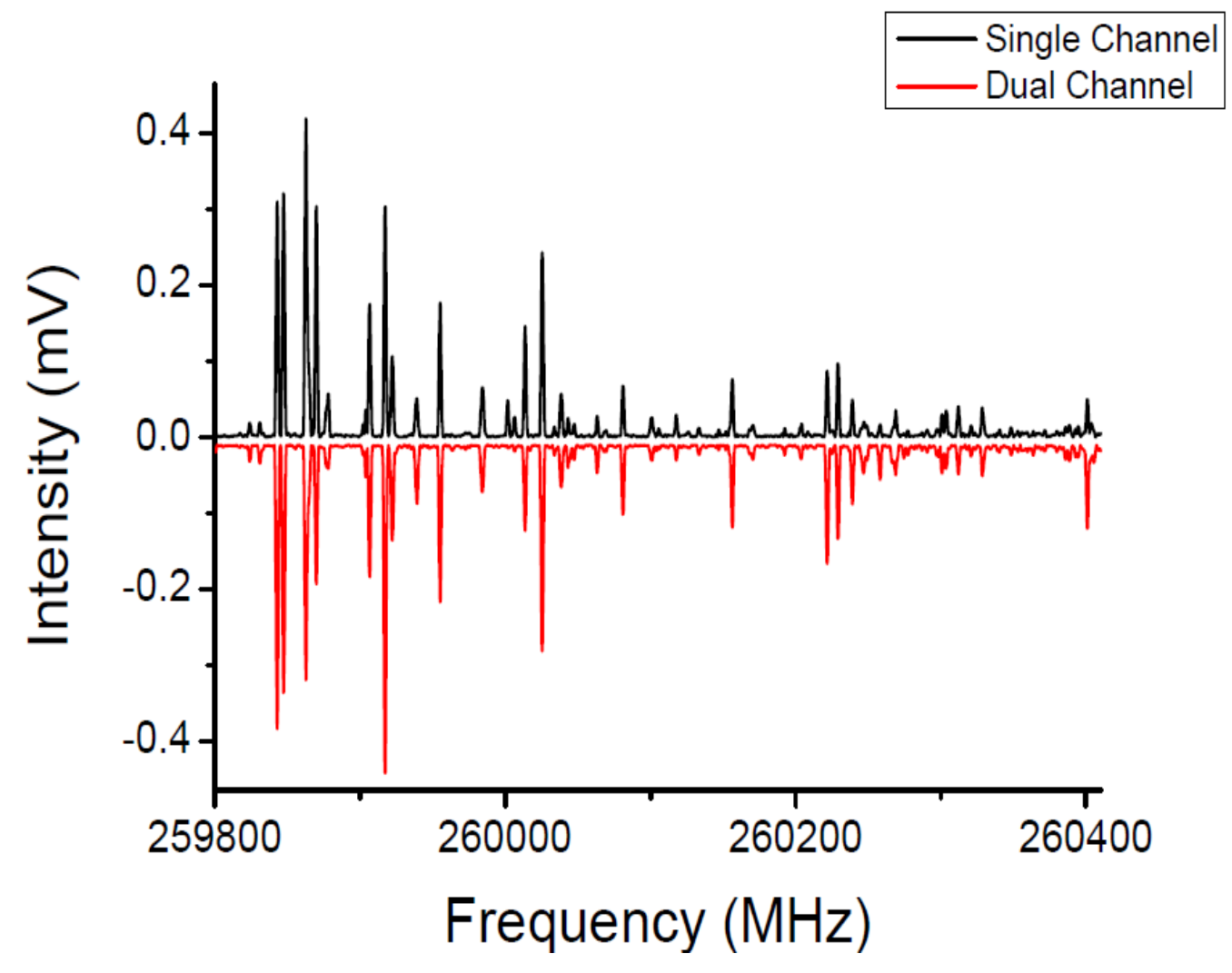

Figure 2.4. This figure compares the spectrum of ethyl cyanide measured with both the single channel and dual channel setup. A pressure of $0.5 \mathrm{mTorr}$ was used, and 10,000 acquisitions were averaged. 


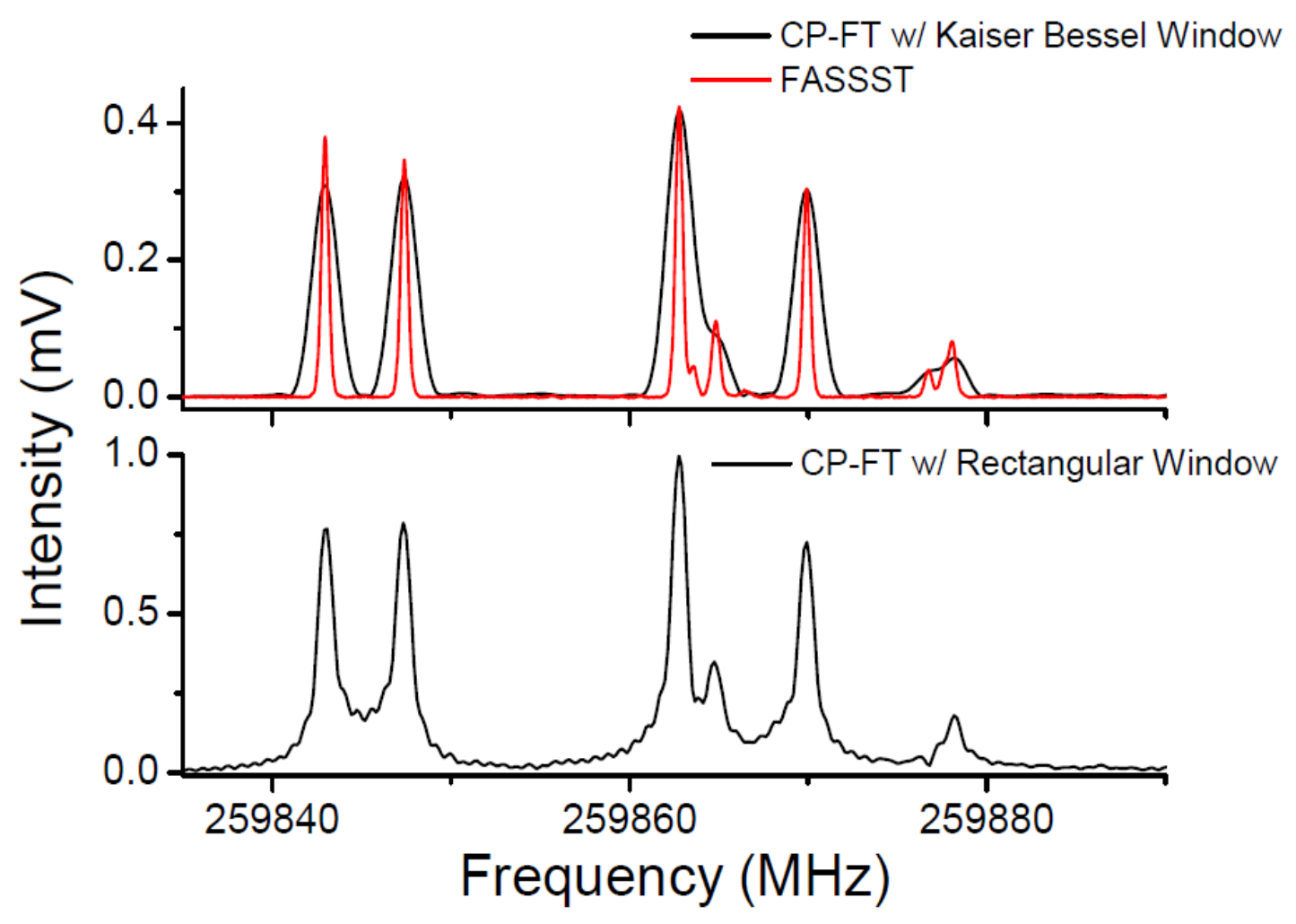

Figure 2.5. This figure illustrates the effect of applying window functions to time domain data. On top is a spectrum of ethyl cyanide after signal processing with a Kaiser Bessel window (FWHM 1.4MHz) and overlaid with the FASSST spectrum; on the bottom a Rectangular window was applied (FWHM 1MHz). Window tapers improve wing suppression of strong signals, which helps to resolve weak emission; however, the FWHM line width increases. 
line width of $460 \mathrm{kHz}$. For the CP-FT measurement under these conditions, the FID decay is dominated by Doppler dephasing. The immediate difference in these spectra is that the CP-FT line width is about 3 times broader.

There are three reasons for the poorer resolution in CP-FT spectroscopy. First, the spectrum is generated by the magnitude Fourier transform. This analysis method includes both the absorption and dispersion contributions in the line shape, which for a Doppler profile increases the full-width at half maximum (FWHM) by about a factor of 2 over the absorption component alone. In addition, there is a delay between the excitation of each transition and the beginning of the FID digitization due to the nature of the chirped pulse excitation (as the frequency sweeps linearly through the $36 \mathrm{GHz}$ of spectral bandwidth) and the dead time for amplifier recovery from saturation (about 200ns). For Doppler dephasing, which has an $\exp \left(-\mathrm{t}^{2}\right)$ decay profile, this time delay further broadens the line shape. The third line broadening contribution comes from the spectral window functions we apply. The direct magnitude Fourier transform of the FID is shown in the lower panel of Fig. 2.5. In this case, the line width is about $1 \mathrm{MHz}$, roughly twice the Doppler limit of the absorption spectrum, as expected from the dead time delay and use of the magnitude FFT. With the inclusion of the dispersion part of the molecular response, the spectrum has significant "wings" that provide poor baseline resolution. The effects of the wings are further complicated by phase interference from nearby transitions that typically distorts the line shape of a weak transition in the wings of a strong one. This effect can be seen for the two transitions near $259878 \mathrm{MHz}$ in Fig. 2.5. Both of these effects can be minimized by using spectral window functions to reduce the "signal 
leakage" of the molecular transitions. We apply a Kaiser-Bessel window function ${ }^{21}$ (with $\beta=8$ ) to the FID average prior to the FFT in our data analysis. This window minimizes the line width at baseline, but increases the full width at half maximum. This data processing step leads to the final result of line widths about a factor of three larger than observed in the FASST absorption spectra.

Ethyl cyanide is a favorable molecule for molecular rotational spectroscopy because it has a large dipole moment $\left(\mu_{\mathrm{a}}=3.84 \mathrm{D}, \mu_{\mathrm{b}}=1.23 \mathrm{D}^{22}\right)$. We have also benchmarked the performance of the spectrometer for the structurally similar 1-butyne $\left(\mu_{\mathrm{a}}=0.76 \mathrm{D}, \mu_{\mathrm{b}}=0.17 \mathrm{D}^{23}\right)$ shown in Fig. 2.6. In this measurement, the sample pressure is 20 mTorr and was adjusted to give the best signal-to-noise ratio without pressure broadening of the line width beyond the instrumental limit (observed FWHM of 1.70 MHz). We have analyzed the rotational spectrum of the ground vibrational state by extending the results of Landsberg and Suenram ${ }^{23}$ and Demaison ${ }^{24}$. Using the rotational constants from these works, it was straightforward to extend the assignment to the 260-295 GHz region, and the results are shown in Appendix A. The results of a fit to the S-reduction Watson Hamiltonian using SPFIT $^{25}$ are presented in Table 2.1. Fit results are shown for the global fit. Because the rotational spectrum of 1-butyne is expected to be well-characterized by the Watson Hamiltonian, the root-mean-squared (rms) frequency difference provides a good measure of the frequency accuracy of the CP-FT spectrometers. Using just the 108 observed transition CP-FT frequencies with the global fit, the rms frequency uncertainty is $35 \mathrm{kHz}-2 \%$ of the experimental full width at half maximum. 

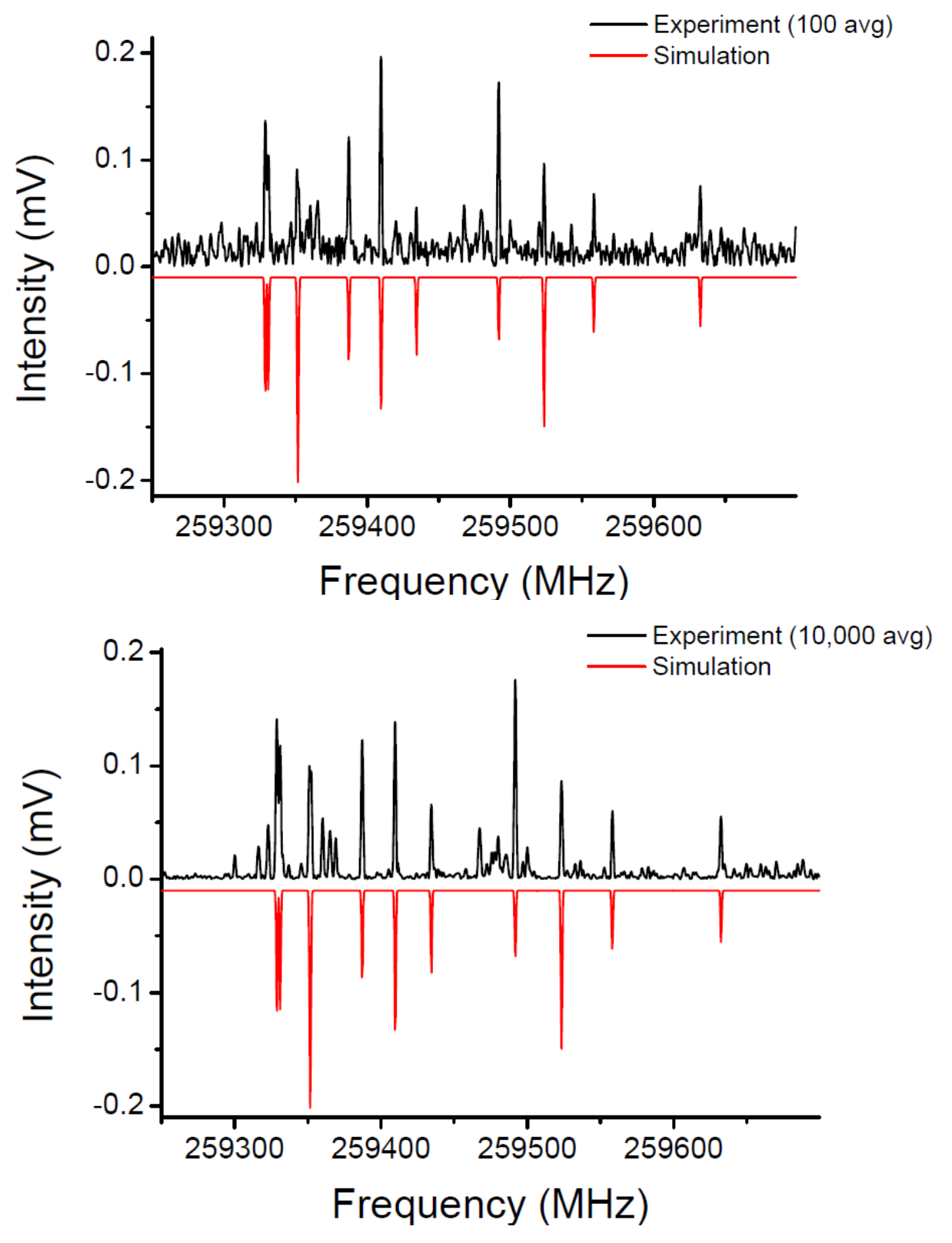

Figure 2.6. Spectrum of 1-butyne taken at $20 \mathrm{mTorr}$ in the single channel configuration. The left panel shows the spectrum after 100 averages, with a $\mathrm{S} / \mathrm{N}$ of $10: 1$, while the right panel shows the spectrum after 10,000 averages and the $\mathrm{S} / \mathrm{N}$ is 117:1. 
Table 2.1. The fitted parameters for 1-butyne using an S-reduction Watson Hamiltonian. These included the transitions from the CP-FT experiment, Landsberg and Suenram ${ }^{23}$, and Demaison et al. ${ }^{24}$.

\begin{tabular}{cc}
\hline Parameter & Global Fit \\
\hline$A(\mathrm{MHz})$ & $27147.7938(5)$ \\
$B(\mathrm{MHz})$ & $4546.51889(49)$ \\
$C(\mathrm{MHz})$ & $4086.91349(49)$ \\
$D_{J}(\mathrm{kHz})$ & $-2.67528(50)$ \\
$D_{J K}(\mathrm{kHz})$ & $45.4179(19)$ \\
$D_{K}(\mathrm{kHz})$ & $-579.54(13)$ \\
$d_{l}(\mathrm{kHz})$ & $-0.63066(30)$ \\
$d_{2}(\mathrm{kHz})$ & $-0.03240(13)$ \\
$H_{J}(\mathrm{~Hz})$ & $0.00746(18)$ \\
$H_{J K}(\mathrm{~Hz})$ & $-0.11808(90)$ \\
$H_{K J}(\mathrm{~Hz})$ & $-1.4284(20)$ \\
$H_{K}(\mathrm{~Hz})$ & $33.1(10)$ \\
$h_{l}(\mathrm{~Hz})$ & $0.00342(16)$ \\
$h_{2}(\mathrm{~Hz})$ & $0.000455(75)$ \\
$h_{3}(\mathrm{~Hz})$ & $0.000052(28)$ \\
$\mathrm{RMS}(\mathrm{kHz})$ & 61.8 \\
$\#$ lines & 302 \\
\hline
\end{tabular}




\section{Discussion}

Current high-speed digital electronics are well-matched to high-power, solid-state mm-wave frequency sources for CP-FT spectroscopy. These devices provide spectral bandwidths of about $10-20 \%$ of the center frequency. For the active multiplier system used in this work with a center frequency of $277 \mathrm{GHz}$, the mm-wave source provides $>10 \mathrm{~mW}$ power over a $35 \mathrm{GHz}$ bandwidth (13\%). The full bandwidth of this system can be digitized in a single spectrum acquisition following frequency downconversion using a subharmonic mixer. Our results show that the solid-state sources can achieve the high frequency sweep rates required for this measurement, $1.44 \times 10^{8} \mathrm{GHz} / \mathrm{s}$, with the phase reproducibility required for time-domain signal averaging.

However, there are several performance tradeoffs for CP-FT spectroscopy compared to other mm-wave methods like FASSST. Perhaps the most important is that the current implementation of CP-FT spectroscopy has an instrumental line width about three times larger than observed in absorption spectroscopy. Room-temperature mmwave spectra are typically dense from the contributions of the rotational spectra of thermally populated vibrational states, so resolution can be crucial for spectral assignments. In principle, the CP-FT spectrum can be phase unwrapped to produce the absorption spectrum ${ }^{26}$, and this would make the resolution of the technique similar to absorption spectroscopy. However, we have not been able to implement a phase correction scheme over the full $35 \mathrm{GHz}$ of spectrum bandwidth that avoids numerical artifacts. 
The second performance tradeoff is related to the data throughput of the highspeed (100 GS/s) digitizer used in this work. Data processing limits the measurement duty cycle to about $0.1 \%$ for fast frame averaging and reduces the effective spectrometer sensitivity (for example, measured by the sensitivity achieved in a fixed measurement time period). The data throughput will improve with future advances in high-speed digital electronics and real-time signal acquisition performance will eventually be feasible. For current applications, the reduced measurement duty cycle is relatively unimportant for applications of the technique to observe molecular species that are produced by low duty cycle sources.

\section{Conclusions}

We have demonstrated a CP-FT spectrometer design for mm-wave spectroscopy that is capable of measuring $35 \mathrm{GHz}$ of spectral bandwidth in a total measurement time of $2 \mu \mathrm{s}$. The instrument design uses a single-channel high-speed AWG to generate both the excitation pulse and the local oscillator for the subharmonic mixer and is the only tunable frequency source in the experiment. The CP-FT method uses time-domain signal averaging to increase measurement sensitivity. For ethyl cyanide and 1-butyne, broadband spectra with signal-to-noise ratios of 100:1 and 10:1 (respectively) are obtained in 100 averages with a total measurement time of $0.2 \mathrm{~ms}$. Frequency calibration of the spectrum is performed automatically through the Fourier transform of the digitized time-domain FID signal and the frequency accuracy is ensured by having all signal sources locked to a $10 \mathrm{MHz}$ rubidium time standard. Relative intensity information was 
found to be good by comparison to benchmark measurements from FASSST spectroscopy. Because the chirped-pulse excitation and FID collection steps of the measurement are time separated, the method is essentially background-free and requires no baseline corrections. The ultrashort $2 \mu$ s measurement time for broadband spectrum acquisition and the ability to measure a series of spectra back-to-back in burst mode signal acquisition make this technique well-suited for using mm-wave rotational spectroscopy to capture repeatable transient chemical events like those produced by laser excitation and pulsed sources of highly reactive chemical species. 


\section{Chapter 2 References}

(1) This chapter contains material from a published manuscript: Steber, A. L.; Harris, B. J.; Neill, J. L.; Pate, B. H. J. Mol. Spectrosc. 2012, 280, 3-10.

(2) Brown, G. G.; Dian, B. C.; Douglass, K. O.; Geyer, S. M.; Pate, B. H. J. Mol. Spectrosc. 2006, 238, 200-212.

(3) Brown, G. G.; Dian, B. C.; Douglass, K. O.; Geyer, S. M.; Shipman, S. T.; Pate, B. H. Rev. Sci. Instrum. 2008, 79, 053103.

(4) Shipman, S. T.; Pate, B. H. In Handbook of High-resolution Spectroscopy; John Wiley \& Sons, Ltd, 2011.

(5) McGurk, J. C.; Schmalz, T. G.; Flygare, W. H. J. Chem. Phys. 1974, 60, 41814188.

(6) Steber, A. L.; Neill, J. L.; Zaleski, D. P.; Pate, B. H.; Lesarri, A.; Bird, R. G.; Vaquero-Vara, V.; Pratt, D. W. Faraday Discuss. 2011, 150, 227-242.

(7) Pupalaikis, P. Digitial Bandwidth Interleaving, 2010.

(8) Medvedev, I. R.; Neese, C. F.; Plummer, G. M.; De Lucia, F. C. Opt. Lett. 2010, $35,1533-1535$.

(9) Prozument, K.; Colombo, A. P.; Zhou, Y.; Park, G. B.; Petrović, V. S.; Coy, S. L.; Field, R. W. Phys. Rev. Lett. 2011, 107, 143001.

(10) Drouin, B. J.; Maiwald, F. W.; Pearson, J. C. Rev. Sci. Instrum. 2005, 76, 093113.

(11) Lewen, F.; Gendriesch, R.; Pak, I.; Paveliev, D. G.; Hepp, M.; Schieder, R.; Winnewisser, G. Rev. Sci. Instrum. 1998, 69, 32-39. 
(12) Neill, J. L.; Shipman, S. T.; Alvarez-Valtierra, L.; Lesarri, A.; Kisiel, Z.; Pate, B. H. J. Mol. Spectrosc. 2011, 269, 21-29.

(13) Wilcox, D. S.; Hotopp, K. M.; Dian, B. C. J. Phys. Chem. A 2011, 115, 88958905.

(14) Park, G. B.; Steeves, A. H.; Kuyanov-Prozument, K.; Neill, J. L.; Field, R. W. J. Chem. Phys. 2011, 135, 024202.

(15) Gerecht, E.; Douglass, K. O.; Plusquellic, D. F. Opt. Express 2011, 19, 8973-8984.

(16) Zaleski, D. P.; Neill, J. L.; Muckle, M. T.; Seifert, N. A.; Brandon Carroll, P.; Widicus Weaver, S. L.; Pate, B. H. J. Mol. Spectrosc. 2012, 280, 68-76.

(17) Petkie, D. T.; Goyette, T. M.; Bettens, R. P. A.; Belov, S. P.; Albert, S.; Helminger, P.; Lucia, F. C. D. Rev. Sci. Instrum. 1997, 68, 1675-1683.

(18) Medvedev, I.; Winnewisser, M.; De Lucia, F. C.; Herbst, E.; BiałkowskaJaworska, E.; Pszczółkowski, L.; Kisiel, Z. J. Mol. Spectrosc. 2004, 228, 314-328.

(19) Fortman, S. M.; Medvedev, I. R.; Neese, C. F.; Lucia, F. C. D. Astrophys. J. 2010, $725,1682$.

(20) Pickett, H. M.; Poynter, R. L.; Cohen, E. A.; Delitsky, M. L.; Pearson, J. C.; Müller, H. S. P. J. Quant. Spectrosc. Radiat. Transf. 1998, 60, 883-890.

(21) Nuttall, A. H. IEEE Trans. Acoust. Speech Signal Process. 1981, 29, 84-91.

(22) Heise, H. M.; Lutz, H.; Dreizler, H. Z. Naturforsch. 1974, 29a 1345-1355.

(23) Landsberg, B. M.; Suenram, R. D. J. Mol. Spectrosc. 1983, 98, 210-220.

(24) Demaison, J.; Boucher, D.; Burie, J.; Dubrulle, A. Z Naturforsch 1983, 38a, 447451. 
(25) Pickett, H. M. J. Mol. Spectrosc. 1991, 148, 371-377.

(26) Xian, F.; Hendrickson, C. L.; Blakney, G. T.; Beu, S. C.; Marshall, A. G. Anal. Chem. 2010, $82,8807-8812$. 


\section{Appendix A.}

Table with measured transitions included in the global fit of 1-butyne obtained from the CP-FT mmw instrument from $259.2-295.2 \mathrm{GHz}$.

\begin{tabular}{|c|c|c|c|c|c|c|c|}
\hline $\mathrm{J}^{\prime}$ & $\mathrm{Ka}^{\prime}$ & $K c^{\prime}$ & $J^{\prime \prime}$ & $\mathrm{Ka}^{\prime \prime}$ & $\mathrm{Kc} "$ & $\begin{array}{c}\text { Observed Frequency } \\
(\mathrm{MHz})\end{array}$ & $\mathrm{OMC}$ \\
\hline 30 & 10 & 20 & 29 & 10 & 19 & 259328.77 & 0.011 \\
\hline 30 & 11 & 19 & 29 & 11 & 18 & 259331.06 & 0.022 \\
\hline 30 & 13 & 17 & 29 & 13 & 16 & 259387.17 & 0.037 \\
\hline 30 & 8 & 23 & 29 & 8 & 22 & 259409.67 & -0.014 \\
\hline 30 & 14 & 16 & 29 & 14 & 15 & 259434.41 & 0.044 \\
\hline 30 & 15 & 15 & 29 & 15 & 14 & 259491.78 & 0.019 \\
\hline 30 & 17 & 13 & 29 & 17 & 12 & 259632.48 & 0.030 \\
\hline 30 & 18 & 12 & 29 & 18 & 11 & 259714.22 & 0.022 \\
\hline 30 & 6 & 25 & 29 & 6 & 24 & 259727.65 & 0.017 \\
\hline 30 & 6 & 24 & 29 & 6 & 23 & 259736.77 & -0.035 \\
\hline 30 & 19 & 11 & 29 & 19 & 10 & 259802.78 & -0.052 \\
\hline 30 & 20 & 10 & 29 & 20 & 9 & 259897.92 & -0.042 \\
\hline 30 & 5 & 26 & 29 & 5 & 25 & 260047.27 & -0.007 \\
\hline 30 & 4 & 27 & 29 & 4 & 26 & 260143.60 & -0.019 \\
\hline 30 & 5 & 25 & 29 & 5 & 24 & 260214.40 & -0.004 \\
\hline 30 & 4 & 26 & 29 & 4 & 25 & 261816.37 & -0.014 \\
\hline 31 & 2 & 30 & 30 & 2 & 29 & 262583.00 & 0.001 \\
\hline 30 & 2 & 28 & 29 & 2 & 27 & 263022.14 & -0.033 \\
\hline 31 & 1 & 30 & 30 & 1 & 29 & 263677.39 & -0.044 \\
\hline 32 & 1 & 32 & 31 & 1 & 31 & 264600.77 & -0.017 \\
\hline 32 & 0 & 32 & 31 & 0 & 31 & 264648.93 & -0.027 \\
\hline 30 & 3 & 27 & 29 & 3 & 26 & 264772.80 & -0.017 \\
\hline 31 & 3 & 29 & 30 & 3 & 28 & 267073.26 & -0.026 \\
\hline 31 & 12 & 19 & 30 & 12 & 18 & 267991.32 & 0.029 \\
\hline 31 & 9 & 23 & 30 & 9 & 22 & 268002.67 & 0.038 \\
\hline 31 & 13 & 18 & 30 & 13 & 17 & 268025.57 & 0.020 \\
\hline 31 & 8 & 24 & 30 & 8 & 23 & 268070.22 & 0.016 \\
\hline 31 & 14 & 17 & 30 & 14 & 16 & 268072.64 & 0.051 \\
\hline 31 & 15 & 16 & 30 & 15 & 15 & 268130.48 & 0.013 \\
\hline 31 & 17 & 14 & 30 & 17 & 13 & 268273.67 & -0.023 \\
\hline 31 & 18 & 13 & 30 & 18 & 12 & 268357.31 & -0.031 \\
\hline 31 & 6 & 26 & 30 & 6 & 25 & 268425.79 & -0.009 \\
\hline 31 & 6 & 25 & 30 & 6 & 24 & 268438.89 & 0.006 \\
\hline 31 & 20 & 11 & 30 & 20 & 10 & 268545.87 & -0.048 \\
\hline
\end{tabular}




\begin{tabular}{|c|c|c|c|c|c|c|}
\hline 21 & 11 & 30 & 21 & 10 & 268649.98 & -0.106 \\
\hline 22 & 9 & 30 & 22 & 8 & 268760.44 & -0.016 \\
\hline 31 & 27 & 30 & 5 & 26 & 268767.56 & -0.006 \\
\hline 31 & 28 & 30 & 4 & 27 & 268806.95 & -0.002 \\
\hline 31 & 26 & 30 & 5 & 25 & 268989.01 & 0.014 \\
\hline 32 & 31 & 31 & 2 & 30 & 270805.04 & -0.024 \\
\hline 31 & 27 & 30 & 4 & 26 & 270829.88 & -0.007 \\
\hline 31 & 29 & 30 & 2 & 28 & 271357.47 & -0.038 \\
\hline 32 & 31 & 31 & 1 & 30 & 271743.88 & 0.015 \\
\hline 33 & 33 & 32 & 1 & 32 & 272758.77 & -0.011 \\
\hline 33 & 33 & 32 & 0 & 32 & 272797.25 & -0.014 \\
\hline 31 & 28 & 30 & 3 & 27 & 273713.18 & -0.025 \\
\hline 32 & 30 & 31 & 3 & 29 & 275500.89 & -0.023 \\
\hline 11 & 21 & 31 & 11 & 20 & 276614.08 & 0.055 \\
\hline 10 & 22 & 31 & 10 & 21 & 276619.53 & -0.002 \\
\hline 12 & 20 & 31 & 12 & 19 & 276630.19 & 0.004 \\
\hline 32 & 24 & 31 & 9 & 23 & 276654.50 & 0.027 \\
\hline 13 & 19 & 31 & 13 & 18 & 276663.21 & 0.031 \\
\hline 32 & 25 & 31 & 8 & 24 & 276732.06 & 0.029 \\
\hline 15 & 17 & 31 & 15 & 16 & 276768.10 & 0.022 \\
\hline 16 & 16 & 31 & 16 & 15 & 276836.35 & -0.012 \\
\hline 18 & 14 & 31 & 18 & 13 & 276999.05 & -0.052 \\
\hline 19 & 13 & 31 & 19 & 12 & 277092.15 & -0.018 \\
\hline 32 & 27 & 31 & 6 & 26 & 277127.61 & -0.039 \\
\hline 20 & 12 & 31 & 20 & 11 & 277192.29 & -0.076 \\
\hline 32 & 29 & 31 & 4 & 28 & 277458.15 & 0.002 \\
\hline 32 & 28 & 31 & 5 & 27 & 277489.39 & 0.008 \\
\hline 32 & 27 & 31 & 5 & 26 & 277779.58 & 0.096 \\
\hline 33 & 32 & 32 & 2 & 31 & 279016.11 & -0.022 \\
\hline 32 & 30 & 31 & 2 & 29 & 279633.75 & -0.048 \\
\hline 33 & 32 & 32 & 1 & 31 & 279815.68 & -0.048 \\
\hline 32 & 28 & 31 & 4 & 27 & 279874.38 & -0.004 \\
\hline 34 & 34 & 33 & 1 & 33 & 280914.75 & -0.014 \\
\hline 34 & 34 & 33 & 0 & 33 & 280945.44 & -0.007 \\
\hline 32 & 29 & 31 & 3 & 28 & 282610.41 & -0.018 \\
\hline 33 & 31 & 32 & 3 & 30 & 283906.79 & -0.019 \\
\hline 11 & 22 & 32 & 11 & 21 & 285255.12 & 0.057 \\
\hline 10 & 23 & 32 & 10 & 22 & 285265.07 & 0.042 \\
\hline 13 & 20 & 32 & 13 & 19 & 285300.03 & 0.038 \\
\hline 33 & 25 & 32 & 9 & 24 & 285306.96 & 0.050 \\
\hline 14 & 19 & 32 & 14 & 18 & 285346.17 & 0.043 \\
\hline
\end{tabular}




\begin{tabular}{cccccccc}
33 & 8 & 26 & 32 & 8 & 25 & 285395.25 & 0.040 \\
33 & 15 & 18 & 32 & 15 & 17 & 285404.53 & -0.026 \\
33 & 16 & 17 & 32 & 16 & 16 & 285473.65 & 0.002 \\
33 & 17 & 16 & 32 & 17 & 15 & 285552.23 & 0.000 \\
33 & 18 & 15 & 32 & 18 & 14 & 285639.44 & 0.004 \\
33 & 6 & 27 & 32 & 6 & 26 & 285858.85 & 0.016 \\
33 & 22 & 11 & 32 & 22 & 10 & 286063.32 & -0.103 \\
33 & 4 & 30 & 32 & 4 & 29 & 286095.58 & 0.004 \\
33 & 23 & 10 & 32 & 23 & 9 & 286186.28 & -0.076 \\
33 & 5 & 29 & 32 & 5 & 28 & 286211.95 & 0.079 \\
33 & 5 & 28 & 32 & 5 & 27 & 286587.86 & 0.000 \\
34 & 2 & 33 & 33 & 2 & 32 & 287217.18 & -0.025 \\
33 & 2 & 31 & 32 & 2 & 30 & 287853.78 & -0.019 \\
34 & 1 & 33 & 33 & 1 & 32 & 287893.92 & 0.004 \\
33 & 4 & 29 & 32 & 4 & 28 & 288946.30 & -0.015 \\
35 & 1 & 35 & 34 & 1 & 34 & 289068.83 & -0.033 \\
35 & 0 & 35 & 34 & 0 & 34 & 289093.28 & -0.001 \\
33 & 3 & 30 & 32 & 3 & 29 & 291460.02 & -0.029 \\
34 & 3 & 32 & 33 & 3 & 31 & 292291.12 & -0.011 \\
34 & 11 & 23 & 33 & 11 & 22 & 293895.79 & 0.011 \\
34 & 12 & 22 & 33 & 12 & 21 & 293906.23 & 0.061 \\
34 & 10 & 24 & 33 & 10 & 23 & 293910.65 & 0.056 \\
34 & 13 & 21 & 33 & 13 & 20 & 293936.00 & 0.039 \\
34 & 9 & 26 & 33 & 9 & 25 & 293960.05 & 0.093 \\
34 & 14 & 20 & 33 & 14 & 19 & 293981.45 & 0.074 \\
34 & 15 & 19 & 33 & 15 & 18 & 294039.91 & 0.045 \\
34 & 8 & 27 & 33 & 8 & 26 & 294059.83 & 0.049 \\
34 & 16 & 18 & 33 & 16 & 17 & 294109.69 & 0.046 \\
34 & 17 & 17 & 33 & 17 & 16 & 294189.46 & 0.024 \\
34 & 19 & 15 & 33 & 19 & 14 & 294375.51 & -0.007 \\
34 & 6 & 29 & 33 & 6 & 28 & 294542.38 & 0.045 \\
34 & 4 & 31 & 33 & 4 & 30 & 294717.71 & 0.035 \\
34 & 5 & 30 & 33 & 5 & 29 & 294934.04 & -0.008 \\
26 & 4 & 23 & 25 & 5 & 20 & 21991.030 & -0.153 \\
\hline & & & & & & &
\end{tabular}




\section{Chapter 3}

\section{A Two-Color, Time-Domain Spectrometer for Fourier Transform mm-wave Rotational Spectroscopy}

\section{Introduction}

The room-temperature molecular rotational spectrum of a low pressure gas has a peak spectral intensity in the mm-wave region of the spectrum for polyatomic molecules with two to eight heavy atoms (non-hydrogen atoms) ${ }^{1}$. From the rotational spectrum of a molecule, the structural properties of a molecule can be investigated. In addition to the structural studies of molecules, measurements in the mm-wave region are important for applications to astrochemistry ${ }^{2,3}$ and for possible applications of rotational spectroscopy as an analytical chemistry method. Several technological advances have produced a period of rapid innovation in mm-wave spectroscopy. Solid-state light sources based on active frequency multiplier chains (AMC), described in Chapter 2, are now available for spectroscopy at frequencies above $2 \mathrm{THz}^{4,5}$. These sources offer high output power and wide frequency coverage for molecular rotational spectroscopy; for example, $40 \mathrm{~mW}$ peak power is available at 260-295GHz. Even though high output power is available, in the mm-wave range it is often significantly higher than the power required to saturate the molecular rotational transition in a continuous wave (cw) absorption spectroscopy measurement. However, time-domain Fourier transform spectroscopy can make use of the high power levels as opposed to absorption measurements for sensitivity and measurement speed gains. 
The frequency accuracy of the mm-wave source is derived from the microwave frequency input and needs no further frequency stabilization. In addition to high frequency accuracy, the AMC sources are agile light sources and can be coupled with fast switching microwave input sources or arbitrary waveform generators (AWGs) as show in Chapter 2, allowing for instantaneous change from frequency to frequency. For applications based on Fourier transform spectroscopy, the frequency agility makes it possible to implement measurements using pulse sequences (where the pulses are either a single frequency or multiple frequencies) that can provide additional spectroscopic information. For example, with two independently tunable input frequencies it is possible to perform mm-wave double-resonance experiments that can speed the assignment of molecular rotational spectra due to the measurement's high specificity. The intrinsic time-resolved nature of the Fourier transform measurement can be used to study collisional relaxation processes in the gas using pulse echoes. These experiments rely on the same concepts that have been studied and presented in NMR and the optical regime $^{6-9}$. State-to-state collisional energy transfer can also be measured through twocolor pump-probe spectroscopy, and time-domain measurement methods are also suitable for coupling mm-wave spectroscopy to experiments that generate the sample in a pulsed fashion, such as using laser excitation. In combination, the time resolved measurements enhance the analysis of the gas in question and significantly improve the confidence of identification.

A design for an inexpensive (in comparison to previously presented AMC spectrometers), two-color, time-domain mm-wave spectrometer for Fourier transform 
mm-wave spectrometer in the $260-295 \mathrm{GHz}$ frequency range is described. It can be used for molecular identification and potentially for gas sensing. The operating frequency range is well-matched to the peak of the rotational spectrum intensity for roomtemperature gas samples of molecules with two or more heavy atoms ${ }^{1}$. The AMC source used in the spectrometer produces a peak power of more than $30 \mathrm{~mW}^{4,10}$ and is suitable for performing non-linear, coherent spectroscopy including pulse echoes. The spectrometer uses low-cost microwave light sources and digitizer, both with USB control, to give a design that can augment the measurement capabilities of frequency scanning spectrometers that use AMC light sources and heterodyne detection.

\section{Experimental}

a) Spectrometer design

A schematic diagram of the spectrometer is shown in Fig. 3.1. To generate the mm-wave excitation pulse, the spectrometer uses an AMC mm-wave source with a factor of 24 overall frequency multiplication (VDI AMC291). The AMC input frequency range spans from 10.8-12.3 GHz, for a total of $36 \mathrm{GHz}$ of spectral coverage after x24 multiplication. The coherent molecular emission, or free induction decay (FID) is detected following down conversion in a WR3.4 subharmonic mm-wave mixer (VDI MixAMC 156) which has an intermediate frequency (IF) bandwidth that can cover up to $36 \mathrm{GHz}$. The local oscillator (LO) input to the mm-wave mixer is the same 10.8-12.3 GHz frequency range. 


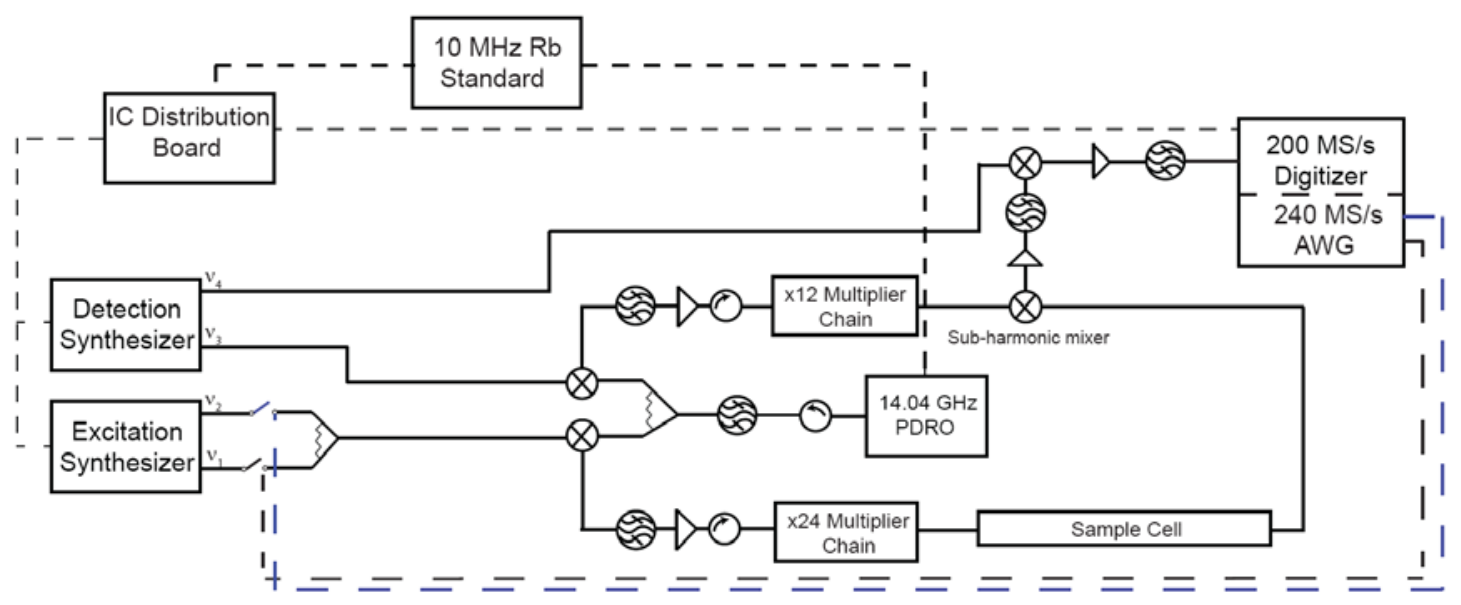

Figure 3.1. Schematic of the dual-color mm-wave instrument operating between 260-290 GHz. The excitation pulse and double resonance pulse are generated from the excitation synthesizer, while the mm-wave LO and second downconversion LO are generated from the detection synthesizer. All frequencies (except for the second downconversion LO) are upconverted to mm-wave frequencies by mixing with the $14.04 \mathrm{GHz}$ PDRO. The signal is digitized by a TiePie HS5, and the switch TTL pulses are generated by two AWGs on the TiePie HS5 combined instrument. 
In order to generate the input frequency for the AMC and MixAMC, a microwave frequency up conversion circuit is used. Microwave sources in the 2-3.5 GHz range are upconverted to the desired AMC and MixAMC input range by mixing with a phaselocked dielectric resonator oscillator (PDRO) at $14.04 \mathrm{GHz}$. The mixer output is filtered using a cavity bandpass filter to select the lower frequency sideband. This microwave signal is subsequently amplified to $15 \mathrm{dBm}$ to drive the mm-wave sources. The tunable microwave input frequency for the AMC and MixAMC LO is provided using two Valon 5008s (Valon Technology), which each have two independent frequency outputs. The Valon 5008 design includes two wideband microwave synthesizer integrated circuit chips with frequency coverage of $2.2-4.4 \mathrm{GHz}$ in its primary output, although the divide-by circuit allows for use below $2.2 \mathrm{GHz}$. The output frequencies and power levels can be set through a USB interface, and each dual-channel synthesizer requires a $10 \mathrm{MHz}$ reference frequency. In order to generate the reference signals a clock distribution integrated circuit (the AD9513 evaluation board) is used. This IC produces three outputs, two complementary metal-oxide-semiconductor outputs (CMOS) for the Valon synthesizers and one low-voltage differential signaling output (LVDS) for the digitizer unit. The clock distribution board input is a single $10 \mathrm{MHz}$ sine wave input from a Rb-disciplined crystal oscillator (Stanford Research Systems). This $10 \mathrm{MHz}$ reference provides both the phase stability and frequency accuracy for the spectrometer.

The receiver system uses two stages to downconvert the molecular emission to a frequency near $65 \mathrm{MHz}$. The mm-wave subharmonic mixer downconverts the FID in the 260-290 GHz range to about $4265 \mathrm{MHz}$. This frequency was chosen due to the second 
mixing stage mixer specifications. This second mixing stage downconverts this signal, following bandpass filtering and amplification, to $65 \mathrm{MHz}$ where it is digitized. We found that a two-stage downconversion had significant advantages over a single stage process where the mm-wave mixer is used to directly generate signals near $65 \mathrm{MHz}$. By moving the IF to a higher frequency, the subharmonic mixer is operating in a more ideal frequency range, while when working at the lower IF frequency of the single stage detection, significant power (potentially due to pulse ringing) was observed at the IF frequency. This caused the baseline to raise and weak signals to be obscured. When a higher IF frequency was used for the mm-wave mixer, the background signal was significantly decreased. Because the Valon synthesizer has two independent microwave outputs, the second output on the synthesizer providing the $\mathrm{LO}$ for the mm-wave mixer is available to provide the LO for the second downconversion step.

Following the downconversion to a frequency near $65 \mathrm{MHz}$, the coherent emission is digitized using a 12-bit, 200 Msample/s digitizer (TiePie, HS 5), which is controlled through a USB interface and has a channel memory depth of 32 million points (Mpts). Built into the unit, the TiePie HS5 also has a 14-bit, 240 Msample/s arbitrary waveform generator (AWG) with $64 \mathrm{Mpt}$ memory depth. A $10 \mathrm{MHz}$ reference (the LVDS clock output from the distribution IC) is integrated into the TiePie HS5, locking both the digitizer and the AWG. The AWG is used to create the pulse pattern needed to control a single-pole, double-throw (SPDT) switch (MiniCircuits ZYSWA-2-50DR). Each output of one of the Valons is controlled by a SPDT in order to create the pulses for either a single color or dual-color experiment. . These created square wave pulses drive 
the AMC excitation source and have a 25 ns time resolution. For dual -color measurements, two separate pulse patterns are required (to drive each of the switches). Thus in the current system, we have combined two TiePie HS5 systems using their internal clock distribution capability to create a measurement system with two AWG channels and four digitizer channels. However, instead of using a dual TiePie instrument, it would also be possible to use a multi-channel pulse pattern generator to create the control signals and compact systems with USB instrument control are available to create high-speed $(100 \mathrm{MHz})$ pulse patterns. Pulse pattern generators such as the USBee ${ }^{11}$ or the Byte Paradigm Wav Gen Xpress ${ }^{12}$ could both be used for this purpose.

The gas cell used for the measurements is a $65 \mathrm{~cm}$ stainless steel tube with a $3.8 \mathrm{~cm}$ diameter and a volume of approximately $725 \mathrm{~mL}$. The ends of the tube (2.75" conflat flanges) are closed off by o-ring seals with Teflon lenses (Thor Labs LAT300 and LAT500) so that the mm-wave radiation can be coupled into and out of the cell with weak focusing, while making a single pass through the tube. Side ports, also using 2.75" conflat flanges, allow sample introduction, pressure monitoring using a capacitance manometer (MKS Baratron, 50 mTorr and 100 Torr heads), and connection to a combination turbo pump and dry scroll pump (Agilent TPS-Compact). The entire spectrometer operates at room-temperature using standard electrical power and requires no water cooling. 


\section{b) Implementation for Fourier transform spectroscopy}

To improve measurement sensitivity through signal averaging in a Fourier transform measurement, it is necessary to add the signals coherently in the time-domain. Because of this, each measurement cycle (the total time of the excitation pulse duration and the subsequent acquisition period) needs to have an excitation pulse with the same phase. As the Valon is a free running source, this can be achieved by using excitation frequencies that contain an integer number of periodic cycles during the measurement cycle. The minimum frequency step at which the synthesizer produces a phase-locked output is a 5 kHzstep size. At the output of the AMC (x24), this gives a frequency resolution of $120 \mathrm{kHz}$ for the spectrometer. This resolution is achieved when the measurement cycle duration is $25 \mu$ s (and integer multiples of this time). Note that this duration is three times longer than the expected measurement cycle time of $8.333 \mu$ s for this frequency resolution $(1 / 120 \mathrm{kHz})$ because it is also required that the measurement cycle time be a rational number. Thus $25 \mu$ s $(8.333 \times 3 \mu$ s) is the shortest time period where $120 \mathrm{kHz}$ frequency resolution can be achieved (the factor of three here is related to the fact that the x24 AMC uses a frequency tripler as one of its components).

For mm-wave rotational spectroscopy of room-temperature gases, the dephasing of the FID is on the order of 700 ns (for molecular mass 60) at lower pressures. For optimum measurement sensitivity, the total gas pressure is adjusted so that the collisional relaxation rate is on the order of the Doppler dephasing (pressures on the order of 10 mTorr). Under these conditions measurement cycles can be repeated in short times after collisional relaxation has re-established equilibrium and coherent excitation effects 
have dephased. We have used a measurement cycle time of $5 \mu \mathrm{s}$ in the spectrometer for signal averaging measurements. With this short measurement cycle time the frequency resolution of the spectrometer is $600 \mathrm{kHz}$. The excitation pulse duration is typically 500 ns in these measurements - a pulse duration shorter than the Doppler dephasing time. The frequency bandwidth of $500 \mathrm{~ns}$ square wave excitation pulse is about $2 \mathrm{MHz}$ so the $600 \mathrm{kHz}$ spectrometer frequency resolution is sufficient to achieve near-resonant excitation. This effect is shown in Fig. 3.2.

The measurement uses the deep memory of the digitizer and AWG channel to acquire multiple signals in one measurement train. With a memory depth of $32 \mathrm{Mpt}$, the digitizer can acquire data for 160 ms. For the $5 \mu$ s measurement cycle, 32,000 signal averages (measurement cycles) can be performed in this single data trace. The AWG output, which creates the excitation pulses by controlling the SPDT switch, and digitizer channel are started simultaneously, and the full sequence of measurement cycles is executed. The digitizer record is then transferred to the computer through the USB interface where the record is partitioned into each measurement cycle and added together in the time-domain through software. The frequency domain spectrum is produced through a fast Fourier transform of the FID. Window functions can be applied to the FID to improve baseline resolution, if desired, as demonstrated in the previous paper ${ }^{13}$ and the cosine transform ${ }^{14}$ can be performed to recover the absorption contribution to the line shape independent from the dispersion as shown in Fig. 3.3. This basic measurement can be used to acquire the spectrum over a frequency range. For example, the use of $25 \mathrm{~ns}$ excitation pulses gives about $40 \mathrm{MHz}$ of measurement bandwidth in each measurement 


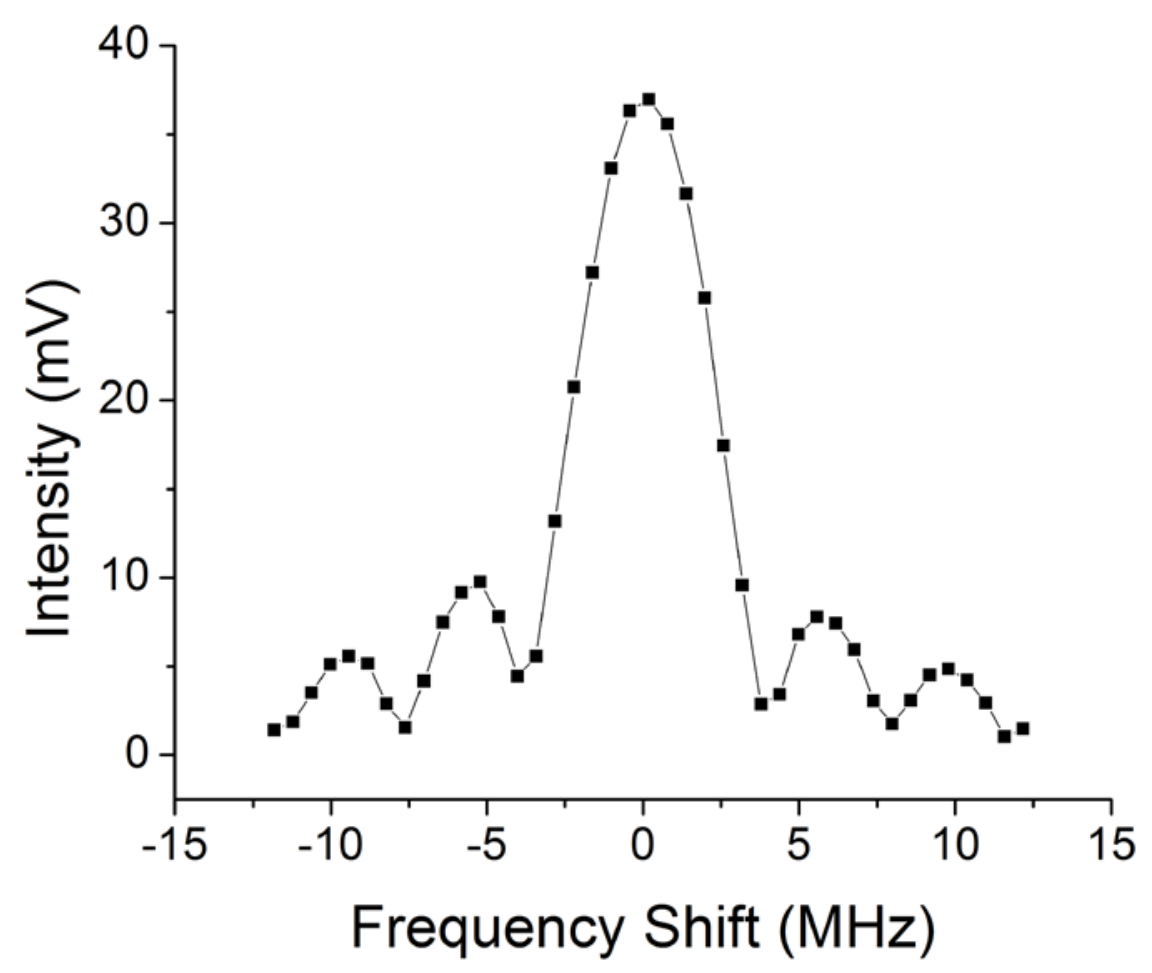

Figure 3.2. Detuning curve for the $\mathrm{J}=22-21$ transition of OCS. The frequency axis indicates how far off the excitation pulse is from resonance, while the intensity axis indicates the transition intensity of the magnitude FT. The frequency resolution of the pulse from the Valons is $25 \mathrm{kHz}$ which equates to $600 \mathrm{kHz}$ in the mm-wave regime. The FWHM is approximately $4 \mathrm{MHz}$, and optimal excitation of the transition can almost be achieved over a $1.2 \mathrm{MHz}$ frequency spread. 

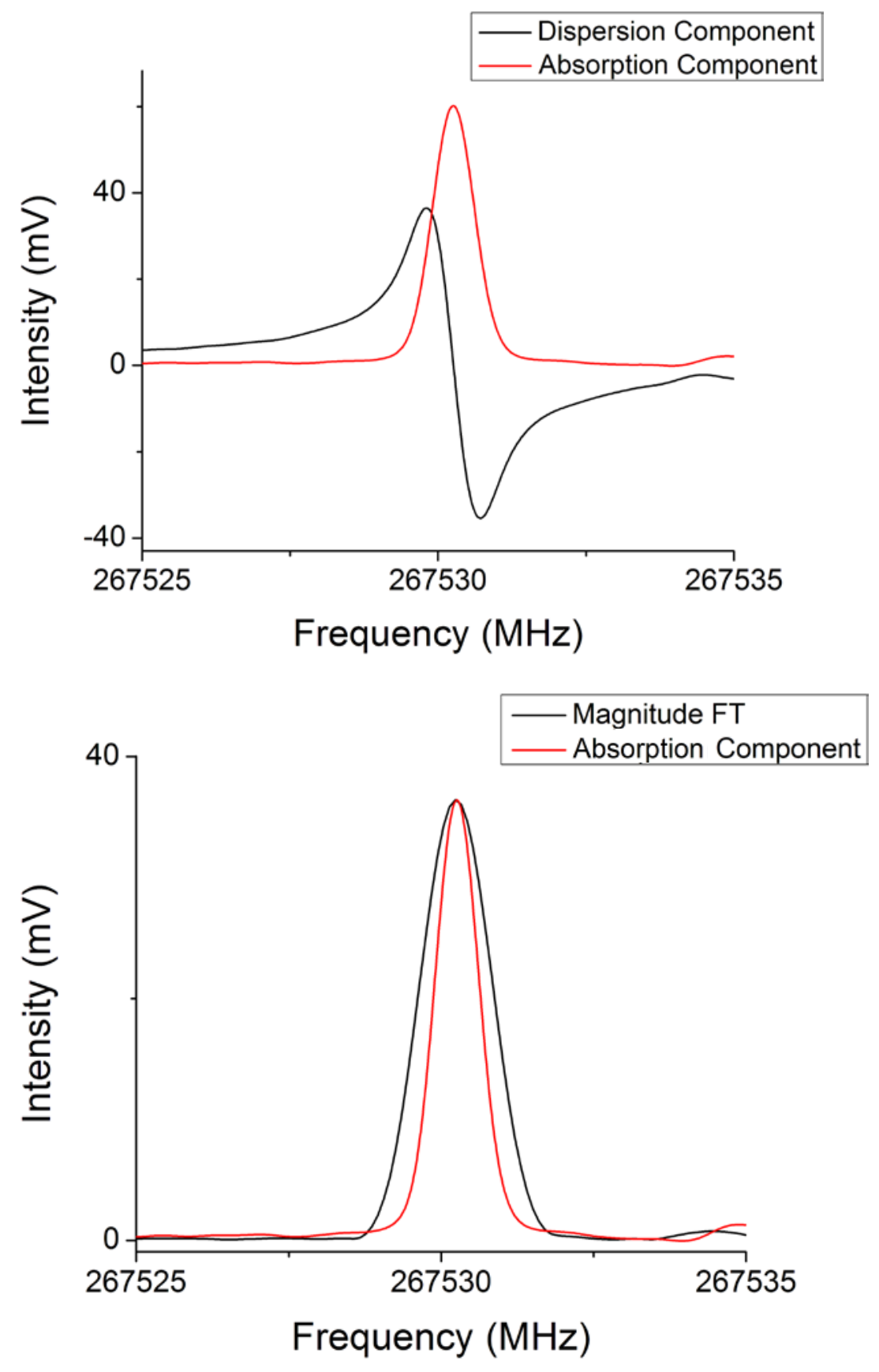

Figure 3.3. The top panel displays the real and imaginary portions of a FT of the OCS $\mathrm{J}=22-21$ transition. In the bottom panel, the cosine component is compared to the magnitude FT which as a FWHM of 1.2 MHz. The FWHM of the absorption component is approximately $900 \mathrm{kHz}$. 
cycle. For this spectrometer, $35 \mathrm{GHz}$ could be covered in 875 "segments". The scanning speed in this mode of operation is limited mainly by the data transfer rate from the digitizer. This is mostly due to the fact that the TiePie HS5 is a USB 2.0 device. The transfer time for a $32 \mathrm{Mpt}$ file is 47s. PC-based digitizer cards, especially those with onboard FPGA capabilities, such as the U1084A Agilent $4 \mathrm{GS} / \mathrm{s}$ real-time digitizer ${ }^{15}$, can dramatically improve the data throughput; however, this would cause a higher expense for the spectrometer digitizer.

\section{Results}

The basic performance of the spectrometer for measuring a single molecular rotational transition is shown in Fig. 3.4. The ${ }^{18} \mathrm{O}^{13} \mathrm{CS}$ isotopologue, which was the lowest abundance isotopologue of OCS observed, was measured at a $\mathrm{S} / \mathrm{N}$ of 13:1 in natural abundance with 10,000 averages at 15 mTorr of pure OCS. This single transition measurement represent 10,000 individual measurement cycles added together in the time domain and then processed to increase the $\mathrm{S} / \mathrm{N}$ by a factor of 100 . The total measurement acquisition time was 20 ms. Single transition measurements can be used in analytical chemistry applications to detect and quantify individual molecular species. However, the main design goal of the instrument is to perform measurements on single molecular rotational transitions that can provide additional spectroscopic characterization beyond the basic frequency domain spectrum. These measurement capabilities can provide information about the collisional relaxation dynamics and can provide important spectroscopic information for analytical chemistry applications of mm-wave rotational 


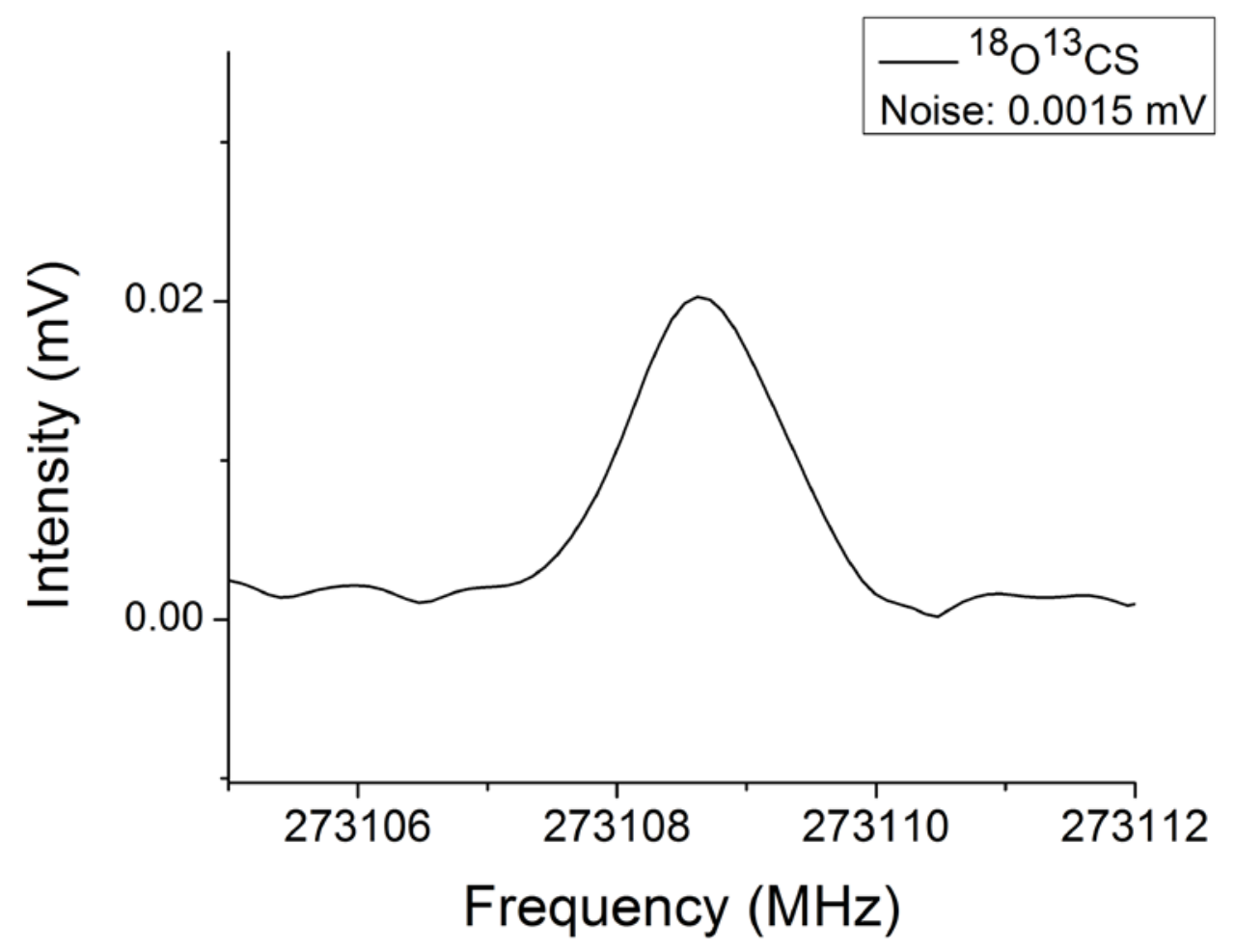

Figure 3.4. The spectrum of the $\mathrm{J}=24-23$ transition of ${ }^{18} \mathrm{O}^{13} \mathrm{CS}$ was measured at $15 \mathrm{mTorr}$ of pressure with a measurement cycle of $5 \mu \mathrm{s}$. The $\mathrm{S} / \mathrm{N}$ of this 10,000 acquisition spectrum is 13:1. Adding the acquisitions in the time domain externally before signal processing produces the same effect as averaging in the digitizer; the noise level fell by a factor of 100. The IF of the line after downconversion is $64.8 \mathrm{MHz}$ which corresponds to a molecular frequency of 273108.65 . 
spectroscopy. Examples of time-domain mm-wave measurements are presented in this section

The measurements presented in this section use coherent excitation to characterize the physical properties of the molecule including the transition moment, the collisional relaxation rate, and the molecular mass. Most of these measurements are performed under lower pressure conditions where the collisional relaxation time is on the order of $20 \mu$ s. Because of the need for complete molecular relaxation and dephasing between successive measurements, measurement cycle durations of about 100 or $150 \mu$ s (multiple of $25 \mu \mathrm{s})$ were used. As a result, the frequency resolution of the spectrometer for these measurements is $120 \mathrm{kHz}$ so that the molecular transition can be excited within +/- $60 \mathrm{kHz}$ of resonance. For all practical purposes, the experiments can be analyzed assuming (exact) resonant excitation. These measurements also use the deep memory feature of the TiePie HS5 AWG and digitizer channel to perform a sequence of measurements in a single data trace. With $100 \mu$ s measurement cycle duration, a total of 1600 back-to-back measurements can be performed in a single 160 ms time window. This ensures constant pressure conditions for experiments that characterize the collision dynamics.

a) Variable pulse duration measurements to characterize the transition moment

The optimization of several measurement sequences, such as photon echoes, requires characterization of the transition moment. The transition moment is characterized by performing a set of successive measurements with incrementing pulse 
duration. A total of 119 measurements with pulse durations from 25 ns to $3 \mu \mathrm{s}$, with 25 ns duration increment, are performed in a single data trace. When $100 \mu$ s measurement cycle times are used, the total measurement time is $11.9 \mathrm{~ms}$ and the record length is $2.38 \mathrm{Mpt}$. The dependence of the molecular signal with pulse duration in a Fourier transform rotational spectroscopy measurement has been presented previously by Campbell and Flygare ${ }^{13}$ and can be described for two systems, a cavity like system or a waveguide system. Only the waveguide system is considered below as our instrument most closely resembles this system.

$$
P_{\text {out }}=\frac{1}{4} \pi\left(\frac{\omega l}{c}\right) \omega_{0}\left(\left|\left\langle a\left|\mu_{z}\right| b\right\rangle\right| \Delta N_{0}\right)^{2} J_{1}^{2}\left(\kappa \varepsilon_{0} \tau_{p}\right) V
$$

where $\kappa$ is defined by:

$$
\kappa=\frac{2}{\hbar}\left|\left\langle a\left|\mu_{z}\right| b\right\rangle\right|
$$

$\mathrm{P}_{\text {out }}$ is the power out, $\mathrm{l}$ is the length of the cell, $\left|\left\langle a\left|\mu_{z}\right| b\right\rangle\right|$ is the transition dipole, $\Delta \mathrm{N}_{0}$ is the population difference, $\mathrm{J}_{1}$ describes the Bessel function, $\tau_{\mathrm{p}}$ is the pulse duration, and $\mathrm{V}$ is cell volume. The signal for a 1 mTorr sample of OCS as a function of the pulse duration is shown in Fig. 3.5 along with the expected functional form of Eq. (3.1). If the mm-wave excitation power has been characterized for the spectrometer, then this measurement can determine the transition moment (and, therefore, the dipole moment component in the principal axis system that gives rise to the transition, as the transition 


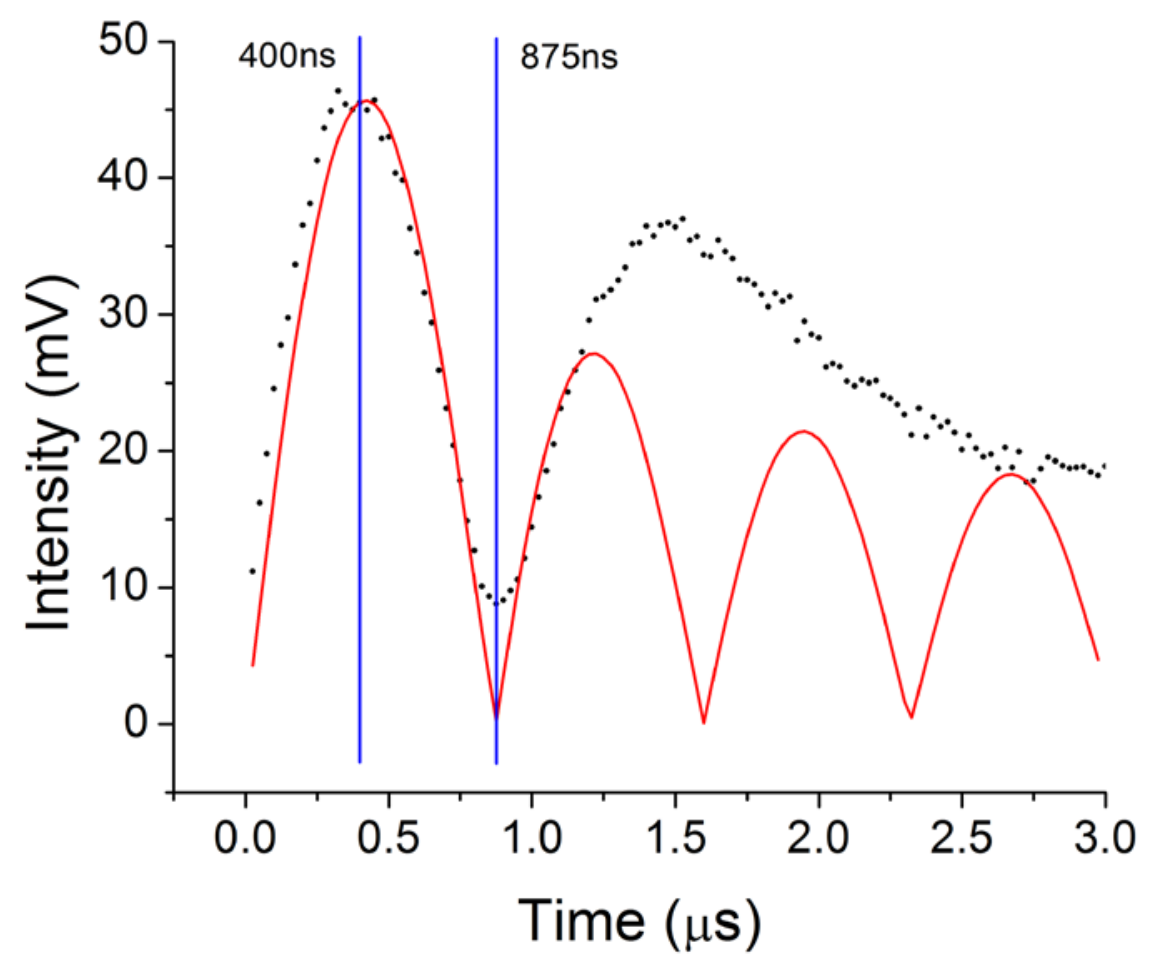

Figure 3.5. The signal level of the $\mathrm{J}=23-22$ transition of OCS versus the pulse duration. The experiment was conducted at 1 mTorr of pressure with $100 \mu$ s measurement cycle times. The $\pi / 2$ pulse duration is $400 \mathrm{~ns}$ while the $\pi$ pulse duration occurs at $875 \mathrm{~ns}$. The red line is a Bessel fit to the experimental data that does not include Doppler dephasing effects. 
moment is roughly half of the permanent dipole moment) for an unassigned transition with unknown molecular carrier.

This result can be interpreted in terms of the coherent excitation of a two-level system using the Bloch model. The optimum signal occurs when the initial population difference is converted into coherence by a " $\pi / 2$ - pulse” where the product of the Rabi frequency (3.9 MHz) and the pulse duration is equal to $\pi / 2$. The signal reaches the first minimum for a pulse duration twice the optimal pulse or a " $\pi$ - pulse" where population inversion is achieved in the two level system. For molecular rotational spectroscopy the two-level system terminology is only approximate (except for transitions involving the ground rotational state) because there are many levels excited (the $\mathrm{M}_{\mathrm{J}}$-degeneracy) each with a different transition moment through the $\mathrm{M}_{\mathrm{J}}$-dependence. Because of this the $\pi$-pulse is only approximately twice the optimal pulse, and the effect of the $\mathrm{M}_{\mathrm{J}^{-}}$ dependence can be seen in the latter half of Fig. 3.5. Also, the spatial dependence of the electric field must be considered when analyzing the latter half of Fig. 3.5. Even though the two-level system nomenclature is an approximation, it will be used below to describe the experiments.

b) Double-resonance spectroscopy and enhanced chemical selectivity in gas analysis

The time-resolved nature of a Fourier transform rotational spectroscopy measurement coupled with the frequency agility of the AMC mm-wave source makes it possible to perform mm-wave double-resonance spectroscopy using a two-color pulse excitation scheme. Under low pressure conditions where coherent excitation is possible, the 
double-resonance measurements can produce signal modulation over 50\% with high frequency selectivity. This method is illustrated in Fig. 3.6 for two sequential rotational transitions of OCS. As the molecular signal is proportional to the population difference, if the population difference is changed, the signal level will also be modulated.

The presented double resonance experiment shows the modulation of the $\mathrm{J}=22-21$ transition (the probe transition) by "pumping” the $\mathrm{J}=23-22$ transition, which is resonant with the $\mathrm{J}=22-21$ transition. The signal level of the $\mathrm{J}=22-21$ rotational transition is affected by a "pump" excitation that is applied before "probe" measurement. In order to achieve maximum change in the probe signal, a $\pi$-pulse is used for the pump pulse to invert the population in the $\mathrm{J}=23-22$ transition. For an ideal two-level system, this would increase the probe signal by $100 \%$ due to a doubling of the population difference. The ratio of the probe signal of OCS at 1 mTorr pressure (as in Fig. 3.5) with a pump pulse to the signal in the absence of a pump pulse is shown in Fig. 3.7. The maximum signal increase is $60 \%$ and occurs for a pulse duration equal to the $\pi$-pulse condition of Fig. 3.5.

This two-color excitation sequence has high frequency selectivity for the doubleresonance signal, which is shown in Fig. 3.8. In this figure the signal modulation caused by the pump pulse at its optimal, $\pi$-pulse duration is shown as a function of detuning from resonance of the $\mathrm{J}=23-22$ pump transition. In order to optimally modulate the signal, the pulse must have a frequency that is $\pm 500 \mathrm{kHz}$ of the resonant frequency of the line, which can be controlled by the measurement cycle length. Like Fig. 3.2, this measurement follows the electric field for a square wave excitation pulse (duration of $875 \mathrm{~ns}$ in this case). If needed, the frequency selectivity can be varied by controlling the 
$J=24$

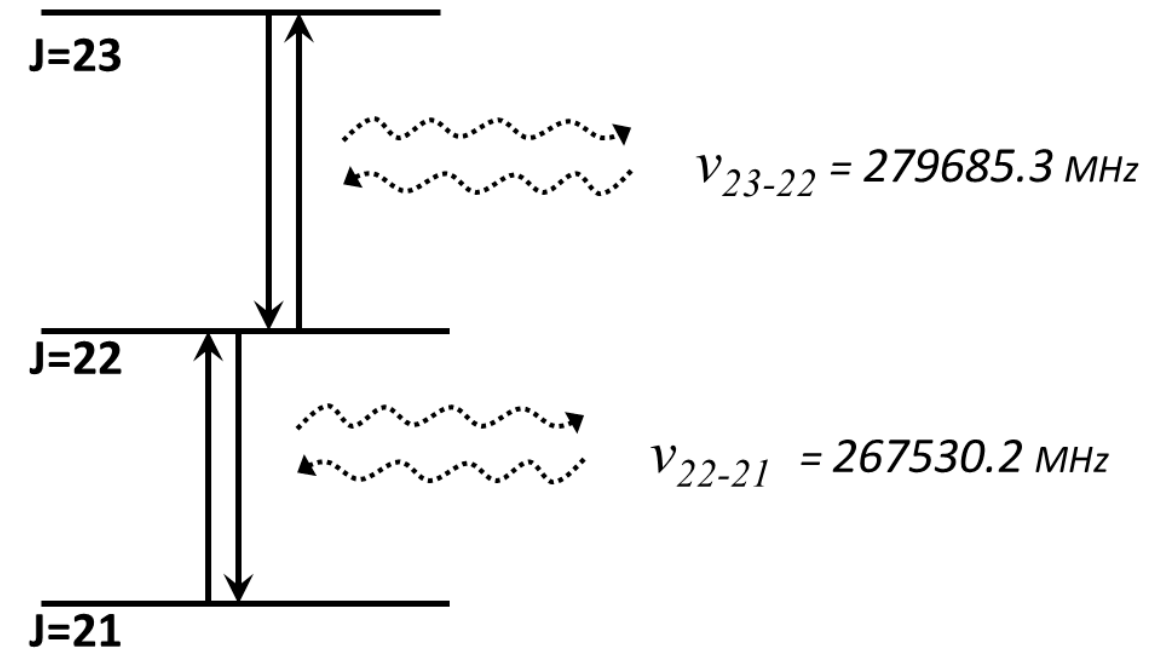

$J=20$

Figure 3.6. The rotational level diagram of OCS showing the available transitions in the 260-290 GHz region. For dual-color experiments a transition that shares a rotational level with the transition of interest (probe transition) is pumped to alter the population difference between the rotational levels of the probe transition. For OCS, we pumped the $\mathrm{J}=23-22$ and monitored the increase in signal of the $\mathrm{J}=22-21$ probed transition. 


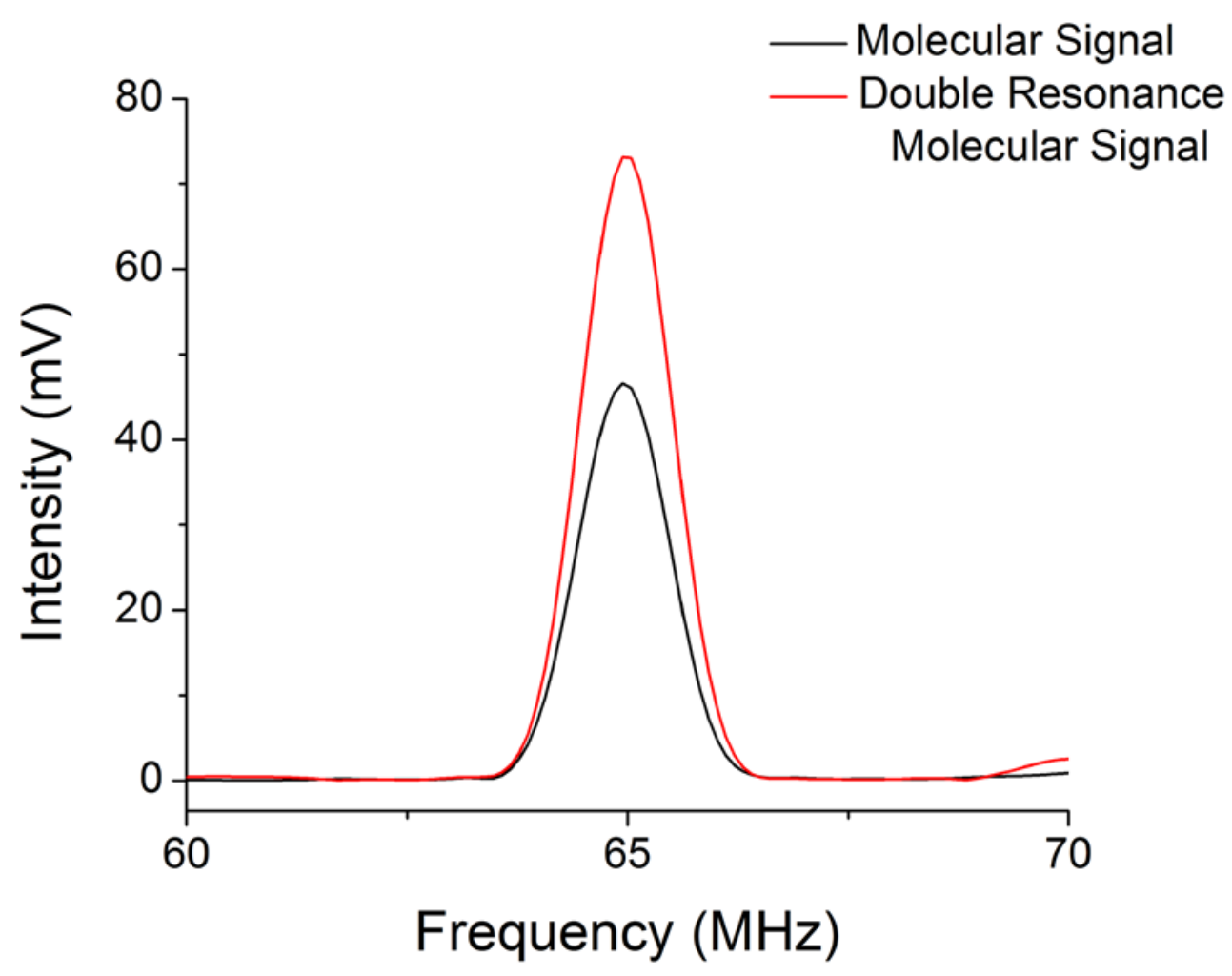

Figure 3.7. This figure illustrates the effects of double resonance on the $J=22-21$ transition (probe transition) of OCS by pumping the $\mathrm{J}=23-22$ transition. The pulse lengths were determined from Fig. 3.5. The pressure was $1 \mathrm{mTorr}$, and the measurement cycle was $25 \mu \mathrm{s}$. A 60\% modulation was seen in the probe transition. 


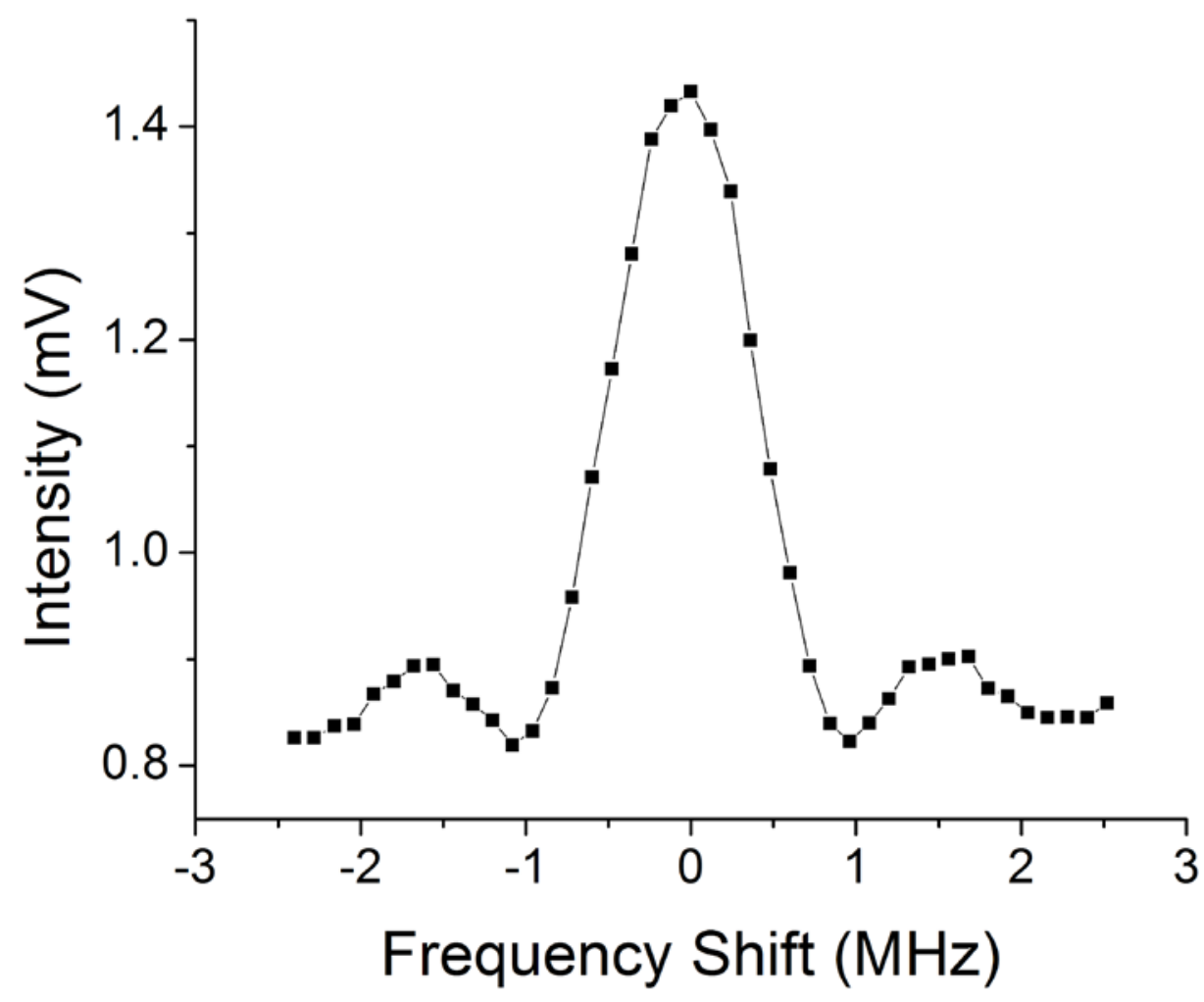

Figure 3.8. Dual-color detuning experiment in which the frequency of the $\pi$ pulse for the $\mathrm{J}=23-22$ transition (pump pulse) was moved through resonance by $60 \mathrm{kHz}$ steps. This figure shows the selectivity of the dual-color measurements. The signal of the probe transition ( $\mathrm{J}=22-21)$ falls as the pump pulse is moved further off resonance. The FWHM is approximately $1.5 \mathrm{MHz}$. 
mm-wave output power so that the pulse duration of the $\pi$-pulse is lengthened as needed (but remains short compared to collisional relaxation times).

Using this kind of experiment can significantly improve the chemical selectivity of mm-wave rotational spectroscopy in trace gas detection for analytical purposes. A problem that is encountered in analytical chemistry applications of room-temperature rotational spectroscopy is that each species in a gas mixture has a dense rotational spectrum that includes rotational transitions in thermally populated vibrational states and the isotopes in natural abundance. The existence of these very large number of weak transitions can make it difficult to use the dynamic range of the spectrometer for weak species detection in complex mixtures. The high density of lines means there is high probability that any single transition of a low abundance molecule will spectrally overlap with the weaker vibrationally excited state and isotopic transitions of a species producing large signals (for high-abundance or large dipole moment). Measurements that identify a molecule in the gas sample by monitoring a few rotational transitions are susceptible to false positive signals when the molecule of interest has low signal levels. However, the double-resonance method improves chemical selectivity because it can verify that the transition detected belongs to the molecule of interest by establishing that it shares a rotational level with another known transition of the same molecule. The high signal modulation (over 50\%) and frequency selectivity of the double-resonance measurement is expected to significantly reduce the probability of a false positive detection and improve the effective dynamic range of trace gas detectors using rotational spectroscopy. 
c) Pulse echoes and collisional relaxation

The high peak power available from AMC sources makes it possible to perform pulse echo experiments to measure the collision relaxation dynamics. Recently pulse echoes for mm-wave spectroscopy have been demonstrated using chirped pulse excitation, and they have been used to study the dynamics in Rydberg states of molecules ${ }^{16}$. The current experiments utilize the Hahn “ $\pi / 2-\tau-\pi$ ” pulse sequence ${ }^{6}$ that is well-known in FT-NMR spectroscopy for spin echo measurements. The pulse durations for the $\pi / 2$ - and $\pi$-pulses are obtained from the transition moment characterization measurement shown in Fig. 3.5.

The time domain pulse echo measurements for the $\mathrm{J}=22-21$ rotational transition of OCS in a 1 mTorr sample are shown in Fig. 3.9. The time delay $(\tau)$ is varied in each successive measurement, resulting in an exponential decay of the signal levels. The top panel shows the echo during the first pulse sequence ( $8 \mu$ s pulse separation for the reduced resolution method) while the bottom panel shows the echo during one of the later pulse sequences (24.8 $\mu$ s pulse separation for the reduced resolution method). A reference measurement cycle is always taken at the beginning of the train so that the mass can be determined using the resulting collisional relaxation time. Fig. 3.10 shows the analysis of the pulse echo experiments. The two panels demonstrate the two ways to perform this experiment. It can be carried out at a high time resolution (Panel A), or the number of measurement cycles can be reduced so that multiple measurement trains can be carried out and averaged together (Panel B). Taking advantage of the memory depth and thus having the ability to average the data can be useful when working with a weak 


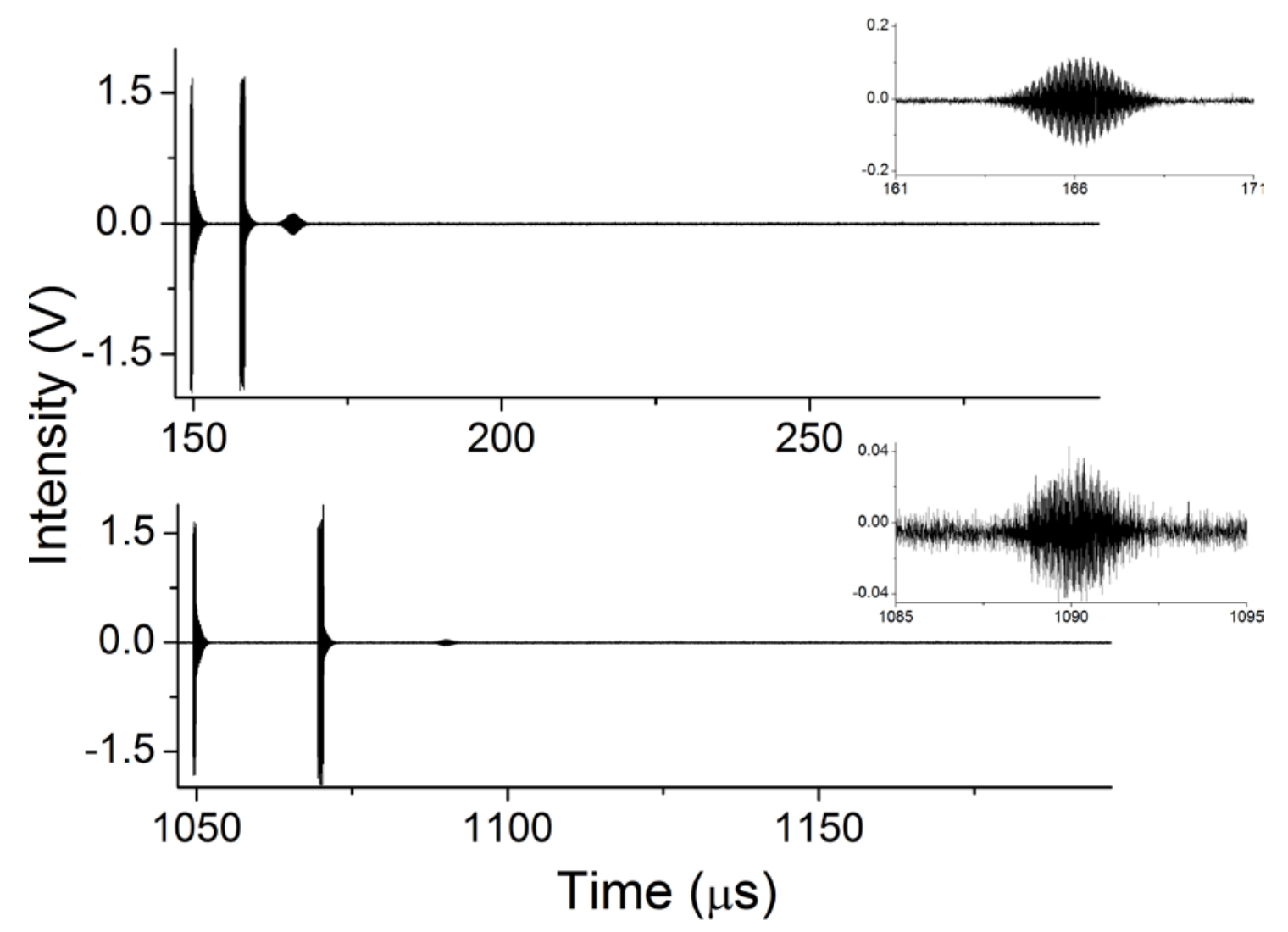

Figure 3.9. Time Domain illustration of the echo experiment. A $\pi / 2$ pulse (determined from Fig. 3.5) is applied and followed by an incrementally increasing amount of deadtime, after which a $\pi$ pulse is applied. The Hahn echo appears at about twice the separation time of the two pulses. 

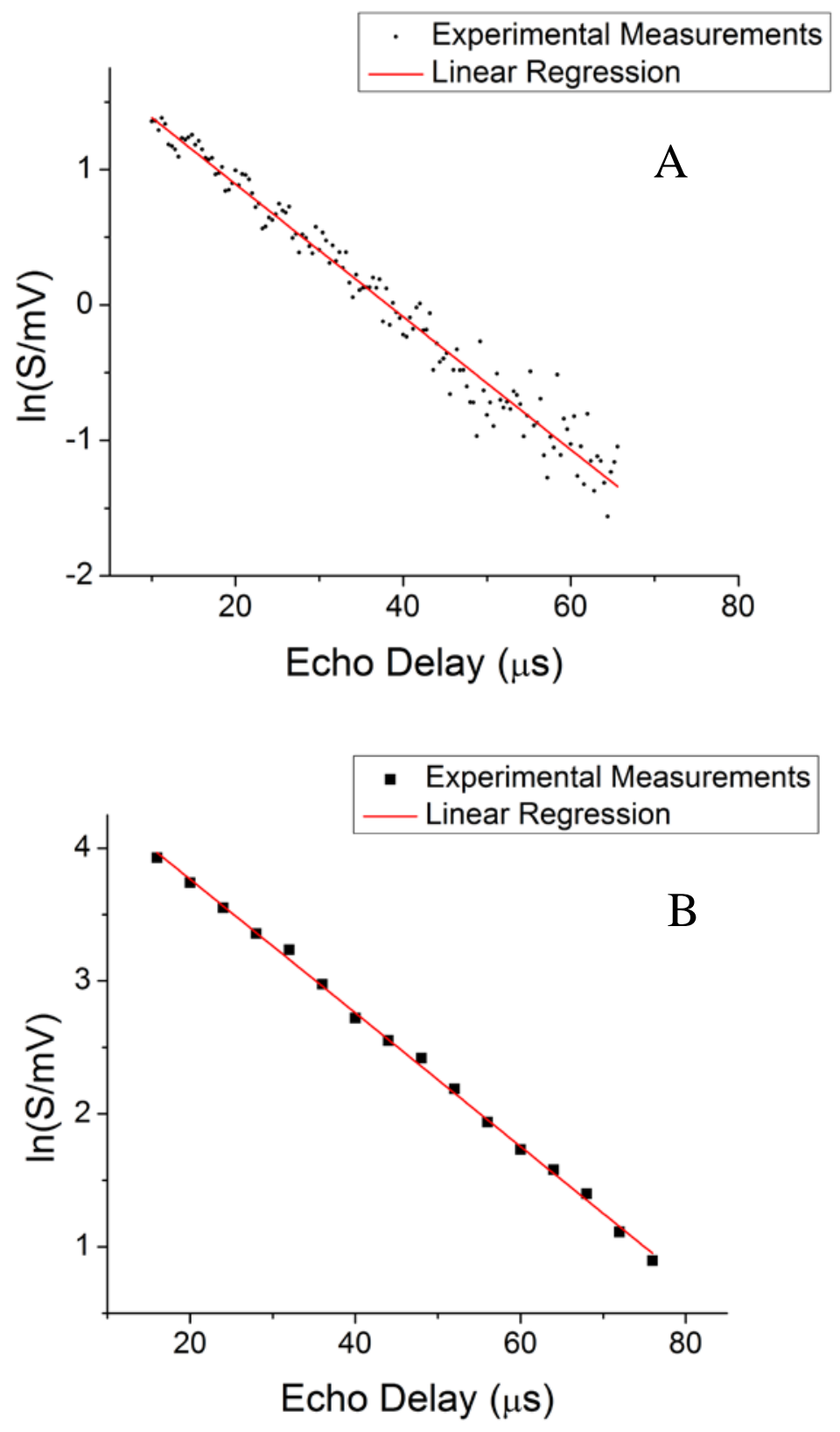

Figure 3.10. Linear regression showing a natural log plot of the echo signal level versus the echo delay. The experiment was performed on the $\mathrm{J}=22-21$ transition of OCS. Panel A shows 130 measurements taken in a single data acquisition at $1 \mathrm{mTorr}$ of pressure for $150 \mu$ s measurement cycles. For each pulse sequence the entire echo signal was Fourier transformed. The resulting collisional dephasing time was determined to be 20.41(30) $\mu \mathrm{s}$. Panel B displays the results of averaging the pulse echoes. The pulse separation time increments were increased, allowing for 64 acquisitions of 16 points. The collisional dephasing time was calculated to be 19.89(21) $\mu$ s. 
species or isotopologue. The experimental implementation for panel A performs a set of 130 echo experiments with pulse delay times (from the beginning of the first pulse to the beginning of the second pulse) from $7 \mu$ s to $33 \mu$ s using a $150 \mu$ s measurement cycle time and increasing by $0.2 \mu$ s for each successive measurement cycle. The total experiment is executed in $19.5 \mathrm{~ms}$ and creates a data record with 3.9 Mpts. The main difference between the first setup and the second is the fact that a measurement train is repeated 64 times for a factor of 8 in the S/N. The pulse delay times are incremented by $2.8 \mu \mathrm{s}$ instead of $0.2 \mu \mathrm{s}$ and a data record length of $28.8 \mathrm{Mpts}$ is generated. The collisional dephasing rate, $\mathrm{T}_{2}$, can be determined from the variation of the echo signal amplitude with the pulse delay time. A collisional dephasing time of 20.41(30) $\mu$ s for panel A and $19.89(21) \mu$ s for panel $B$ is obtained. This gives an average of $20.15(18) \mu$ s. In the earlier literature, Mäder et al. ${ }^{17}$ found the collisional relaxation to be $4.13 \mu$ s at 5 mTorr, which correlates to $20.65 \mu$ s at 1 mTorr. The current data agrees to within $2 \sigma$ of this value. However, in more recent literature where the self-broadening coefficients were reported, the collisional relaxation rate was found to be $23.32 \mu$ s for the $J=23-22$ transitions $^{18}$. Our reported value has an $11 \%$ discrepancy with this calculated rate. Discrepancies between these numbers may reflect the fact that our pressure monitoring system has not been carefully calibrated, and during the course of the experiment we experience degassing of OCS off the walls of the cell so the pressure could not be precisely reported.

The collisional relaxation times were used in order to determine the mass of the molecule. Because the FID has both a Lorentzian component (due to pressure effects) 
and a Gaussian component (due to Doppler broadening), it is important to be able to fit both components. The molecular FID is fit using a program that is based on the approximate equation given by Campbell and Flygare $^{13}$ :

$$
\mathrm{E}(\mathrm{t}) \propto \cos \left(\omega \mathrm{t}_{0}+\omega \mathrm{t}\right) \mathrm{e}^{-\mathrm{t} / \mathrm{T}_{2}} e^{-t^{2} / 4 s^{2}}
$$

In this equation, $\mathrm{s}$ is the Doppler decay constant which is a parameter that is fit with the program and $T_{2}$ is the collisional relaxation time (obtained from the echo experiment). From the results of the fit, the Doppler decay constant is used to determine the mass through the relationship outlined in Campbell and Flygare ${ }^{13}$ :

$$
\begin{gathered}
\mathrm{s}=(\ln 2)^{1 / 2} \Delta \omega_{D}^{-1} \\
\Delta \omega_{D}=\frac{\omega_{0}}{c}\left(\frac{2 k T \ln 2}{m}\right)^{1 / 2}
\end{gathered}
$$

Where $\Delta \omega_{D}^{-1}$ is the Doppler HWHM, $\mathrm{k}$ is the boltzman constant, $\mathrm{T}$ is the temperature, and $\mathrm{m}$ is the mass. When these collisional relaxation times were used to in order to determine the fit of the molecular FID, a mass of 60.50 amu and 59.97 amu was obtained for the respective measurements (the mass of OCS is $60 \mathrm{amu}$ ). Along with these two measurements, it was found that multiple measurements determined the mass to within a $2 \%$ error of the actual mass. 
d) Population recovery and collisional relaxation

For molecular rotational transitions, it is expected that the collisional dephasing $\left(T_{2}\right)$ and lifetime $\left(T_{1}\right)$ are nearly equal, and in multiple studies it has been shown that for

OCS this is the case ${ }^{17,19}$. Because of this, population recovery experiments provide an alternate way to obtain the collisional relaxation time. This measurement can be performed using a simple variation of the two-color double-resonance experiment of Fig. 3.6. In this experiment a "pump” pulse is used to selectively change the population difference between rotational levels, after which collisions re-establish the thermal equilibrium population difference. A probe pulse is applied at a variable time delay following the "pump” pulse to monitor the return of the population to equilibrium. The maximum change in the probe signal level, due to the change in the population difference of the rotational levels, is obtained for a $\pi$-pulse in the pump step - although any pulse duration can be used.

The two-color population recovery signals as a function of the delay between the pump and probe pulses is shown in Fig. 3.11 for the $\mathrm{J}=22-21$ rotational transition of OCS in a 1 mTorr sample (the same sample conditions of the pulse echo experiment). The probe signal is measured with delay times of 200 ns to $25 \mu$ s with increments of $0.2 \mu$ s. The measurement cycle time is $150 \mu$ s and the total of 125 measurements is recorded in a single data trace of $18.75 \mathrm{~ms}$ and record length of 3.75 Mpts. A collisional relaxation time of $20.82(31) \mu$ s is obtained and is in better agreement with the value found by Mäder et al. ${ }^{17}$ than the pulse echo experiment value. The collisional relaxation time was used to determine the mass of the sample using the above described method, and it was 


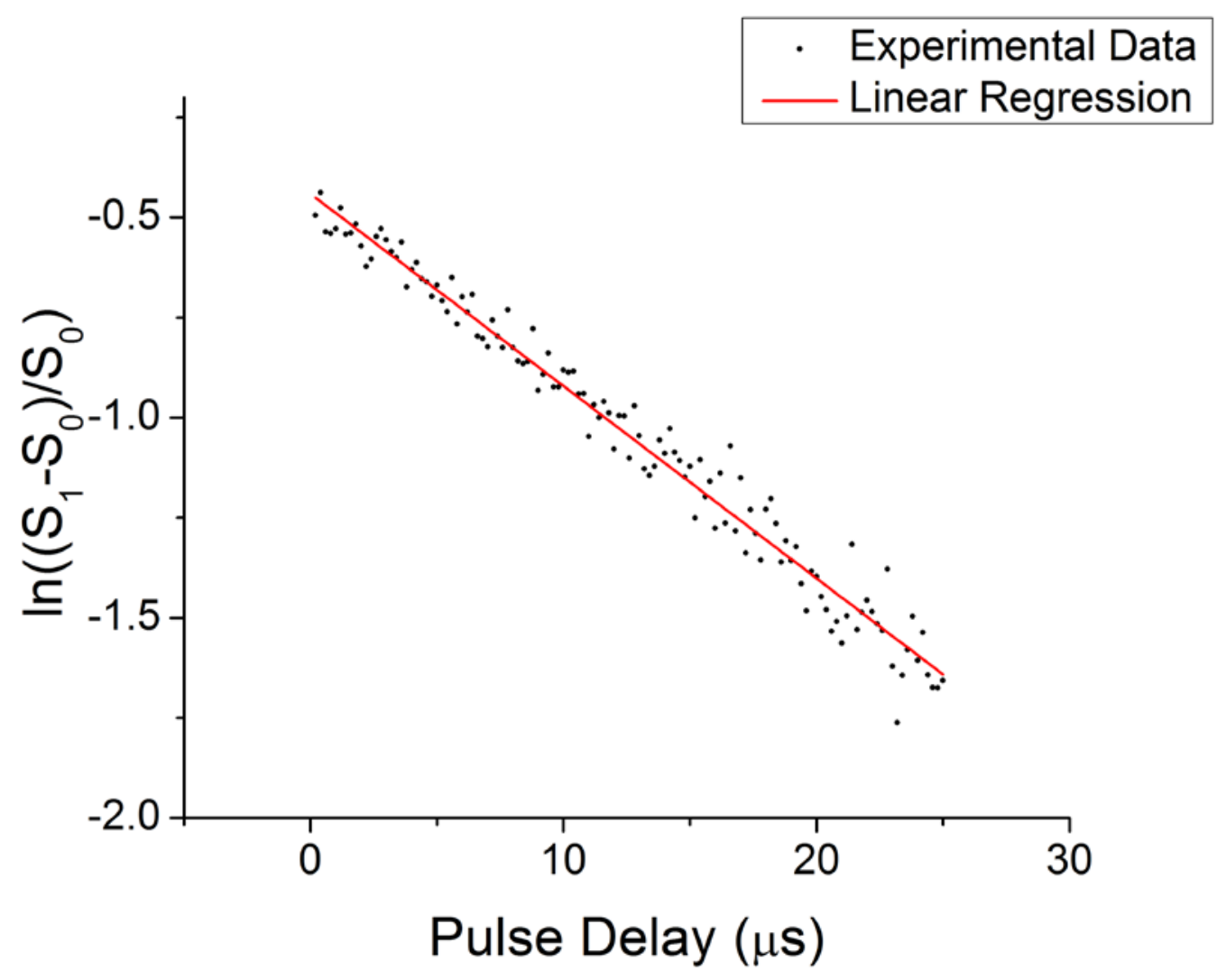

Figure 3.11. The linear regression of the dual-color saturation recovery measurement. The experiment was carried out at 1 mTorr with $150 \mu$ s measurement cycles. For the $\mathrm{J}=$ 22-21 transition of OCS 125 consecutive measurements in which the pulse delay between the $\pi$ pulse and the 200 ns probe pulse were incrementally increased. The resulting collisional dephasing time was calculated to be 20.82(31) $\mu$ s. 
determined to be 59.85 amu. Similar experiments also yielded a molecular mass to within $2 \%$ error of the actual mass. The two-color population recovery experiments have the advantage over the pulse echo experiment in that the signal levels are higher. Because up to nearly $100 \%$ signal modulation is possible with the pump pulse, any transition that can be observed in the spectrum is suitable as a probe pulse. Its disadvantage is that it requires knowledge of the assigned rotational spectrum so that two transitions with a common rotational level can be identified as pump and probe transitions.

\section{Discussion}

The instrument described above is an inexpensive augmentation to AMC based spectrometers. Because the pulse duration can be varied, thus narrowing or widening the pulse sweep, this instrument can also perform a wideband scan in order to find a molecular transition with an unknown molecular frequency. The inexpensive light sources and detectors make this ideal to potentially be developed into a gas sensing instrument to be taken into the field to monitor known molecular species. As such the spectrometer provides new measurement capabilities for applying millimeter wave spectroscopy analytical chemistry applications. By conventional means, molecular lines can be identified by comparison to spectral libraries, however, where unassigned lines cannot be unambiguously matched to known spectra the techniques discussed above can aid the assignment. With development of high throughput digitizers, multiple measurements under the same conditions (in a static cell) can be collected in real-time without the processing time during the data collection being a hindrance. This opens up 
the doors to trace gas detection without sample destruction, and the identification of unknown molecules by determining the transition dipole moment for abundance estimation and the collisional relaxation time for mass estimation.

The instrument must have high sensitivity to measure weak emission features for trace detection applications. The TiePie HS-5 has the capability to measure a record length that corresponds to $160 \mathrm{~ms}$, which opens up the door to better time resolved measurements such as in Figs 3.10 and 3.11 for the measurement of the relaxation coefficient, or to signal averaging, as seen Fig. 3.2. OCS is a typical spectrometer benchmark molecule for rotational spectroscopy studies. It has a fairly simple spectrum, and in the region from 260-295 GHz, there are three available transitions. With this instrument, the ${ }^{18} \mathrm{O}^{13} \mathrm{CS}$ isotopologue was measured in natural abundance with a $\mathrm{S} / \mathrm{N}$ of approximately 13:1 by collecting and averaging 10,000 spectra. The effective sensitivity of this instrument is roughly $1.5 \mathrm{ppm}$ (due to the memory limitations of the digitizer) which can be improved by sampling conditioning such as preconcentration. If the molecule is already known, a library comparison can be made to determine the molecular identification; however, in cases where there is no match to the library or only a single transition is available, further testing can be done. While the same experiment could be performed using the chirped pulse technique (by narrowing up the bandwidth), an expensive AWG would be required. Using this spectrometer design to augment a preexisting AMC spectrometer eliminates the need for an AWG by using the frequency agile dual channel synthesizer. 
Once an unknown molecular transition (or set of transitions) has been identified in the spectrum, it is further characterized by the variable pulse duration experiment. From the experiment it was found that for OCS the optimal pulse ( $\pi / 2$ pulse) was found to be on average 400 ns as seen in Fig. 3.5. In that same figure it can be seen that the $\pi$ pulse, or the pulse needed for population inversion, was found to be $875 n$. Using this information, the molecules transitional dipole moment could be calculated. Furthermore, by knowing the $\pi / 2$ and $\pi$ pulse durations, the sample can be optimally excited for later characterization where signal levels are weak, such as in double resonance or pulse echo experiments.

In an effort to validate a potential spectrum, double resonance measurements may be performed in order to determine the connectivity of the spectral lines and reduce false identifications resulting from coincidental overlap. This is particularly important in situations of high spectral density. Utilizing the pulse lengths that were obtained from the pulse duration experiments, the signal is can be modulated by $60 \%$ as seen in Fig. 3.7. Since the pulses being used are transform limited and have an inherent bandwidth, it is key to make sure that the instrument has high frequency selectivity so that other molecular transitions (other than the ones being probed) are not modulated.. The results of this experiment are seen in which is seen in Fig. 3.8, and the linked rotational transitions can be mapped out and previously unknown spectra assigned relatively quickly. This is especially the case with this instrument as every component can be controlled through USB. The list of transitions to test can be programmed and cycled through in a quick manner. 
The collisional relaxation measurements provide further and final characterization of the molecule. The collisional relaxation time was found to be 20.41(30) $\mu$ s and 20.82(31) $\mu$ s for the echo and dual-color saturation experiments respectively. When these measurements were used to fit the FID of the molecule, a mass of $60.5 \mathrm{amu}$ and 59.85amu was obtained for the respective measurements. Using independent measurements, the fit mass reliably was determined to within $2 \%$ error of the molecular mass of OCS. Even though this method is still in development, it holds great promise to being able to identify unknown molecular components in a gas mixture. Mass estimates coupled with the fact that the dipole moment projections can be determined from the variable pulse duration experiments once the electric field within the sample cell is known, opens the opportunity for molecular identification. Since rotational spectroscopy is a selective method which relies on the mass, dipole moment, and orientation of the mass, knowing two out of the three would narrow down the likely candidates. This coupled with a previous knowledge of what might be present could lead to almost certain identification, lending this instrument to be a powerful tool in analytical chemistry. The promise of this instrument is for a sample in, answer(s) out approach in which this instrument, along with the described set of experiments, would allow an investigator to sift through a dense spectrum and catalog the molecular components.

\section{Conclusions}

A design for a spectrometer using AMC mm-wave sources and heterodyne detection with subharmonic mm-wave mixers in which two-color, Fourier transform mm- 
wave spectroscopy can be performed has been described. This spectrometer uses lowcost, USB-controlled components for pulse generation and FID detection making it a practical addition to existing spectrometers. Because of the long data memory available in the digitizer/AWG device, it is possible to perform a sequence of back-to-back measurements in a single data collection event. This capability can improve data analysis in cases where the successive experiments involve incrementing pulse durations or pulse delays - to characterize collision dynamics, for example - by completing the measurement on time scales shorter than pressure or temperature variations in the sample. From this ability comes new ways to characterize the physical properties of molecules using a single rotational transition or, when more information about the spectrum is available, through double-resonance measurements using two transitions with a shared rotational energy level. The double-resonance technique can aid the assignment of molecular spectra including perturbed spectra that often occur in thermally populated vibrational states. Utilizing and applying the techniques that this instrument is capable of will be useful in gas analysis applications by providing higher chemical selectivity in trace gas monitoring applications and making it possible to characterize the physical properties of an unknown molecule in a gas mixture including its dipole moment and molecular mass. 


\section{Chapter 3 References}

(1) Gordy, W.; Cook, R. L. Microwave Molecular Spectra; 3rd ed.; John Wiley and Sons: New York, 1984.

(2) Fortman, S. M.; Medvedev, I. R.; Neese, C. F.; Lucia, F. C. D. Astrophys. J. 2010, 725, 1682.

(3) Fortman, S. M.; McMillan, J. P.; Neese, C. F.; Randall, S. K.; Remijan, A. J.;

Wilson, T. L.; De Lucia, F. C. J. Mol. Spectrosc. 2012, 280, 11-20.

(4) Crowe, T. W.; Bishop, W. L.; Porterfield, D. W.; Hesler, J. L.; Weikle, R. M. IEEE J. Solid-State Circuits 2005, 40, 2104-2110.

(5) Drouin, B. J.; Maiwald, F. W.; Pearson, J. C. Rev. Sci. Instrum. 2005, 76, 093113.

(6) Hahn, E. L. Phys. Rev. 1950, 80, 580-594.

(7) Kurnit, N. A.; Abella, I. D.; Hartmann, S. R. Phys. Rev. Lett. 1964, 13, 567-568.

(8) Abella, I. D.; Kurnit, N. A.; Hartmann, S. R. Phys. Rev. 1966, 141, 391-406.

(9) Brewer, R. G.; Shoemaker, R. L. Phys. Rev. Lett. 1971, 27, 631-634.

(10) Steber, A. L.; Harris, B. J.; Neill, J. L.; Pate, B. H. J. Mol. Spectrosc. 2012, 280, 310.

(11) USBee QX Test Pod http://www.usbee.com/qx.html.

(12) Leens, F. Wave Gen Xpress - Digital Pattern Generator http://www.byteparadigm.com/products/wave-gen-xpress/ (accessed Nov 19, 2014).

(13) Campbell, E. J.; Buxton, L. W.; Balle, T. J.; Flygare, W. H. J. Chem. Phys. 1981, 74, 813-828. 
(14) McGurk, J. C.; Mäder, H.; Hofmann, R. T.; Schmalz, T. G.; Flygare, W. H. J. Chem. Phys. 1974, 61, 3759-3767.

(15) Harris, B. J. A Chirped Pulse Fourier Transform Millimeter Wave Spectrometer for Room Temperature, Gas Mixture Analysis. Doctor of Philosophy, University of Virginia, 2014.

(16) Colombo, A. P.; Zhou, Y.; Prozument, K.; Coy, S. L.; Field, R. W. J. Chem. Phys. 2013, 138, 014301.

(17) Mäder, H.; Ekkers, J.; Hoke, W.; Flygare, W. H. J. Chem. Phys. 1975, 62, 43804387.

(18) Koshelev, M. A.; Tretyakov, M. Y.; Rohart, F.; Bouanich, J.-P. J. Chem. Phys. 2012, 136, 124316.

(19) McGurk, J. C.; Hofmann, R. T.; Flygare, W. H. J. Chem. Phys. 1974, 60, 29222928. 


\section{Chapter 4}

\section{A Design of a Low-Cost Microwave Spectrometer Operating in the 18-26 GHz Frequency Range}

\section{Introduction}

Rotational spectroscopy provides unique features that make it extremely wellsuited for analytical applications. The sensitive detection limits along with the unambiguous assignment of conformers and isomers, allow a complex sample to be fully analyzed. Besides the sensitive detection limits, coherence "tricks" demonstrated in

Chapter 3, such as nutation measurements ${ }^{1-3}$, detuning measurements ${ }^{4}$, and double resonance $^{5,6}$ measurements can be used to help characterize and identify unknown molecular signatures in a complex sample ${ }^{3}$. In order for the identification of unknown molecules to be feasible, it is important to have a system that has been highly optimized in order to apply it to analytical systems. The sensitivity that is needed for these analytical systems is controlled by the design of the instrument. Super-radiance is an idea that was first presented in the 1950's by Dicke ${ }^{7}$, in which he states that all of the molecules in a system spontaneously radiate together. He also sets forth the relationship that the radiate rate is proportional to the molecular concentration ${ }^{7}$. Another way to think about this is that the intensity of the transitions being monitored is proportional to the square-root of the power of coherent spontaneous emission that is measured. This means 
that it would be advantageous to excite as many molecules as possible, and the design of the instrument should reflect this.

As shown in the introduction, small bandwidth microwave spectrometers have dominated the field until the development of the chirped pulse Fourier transform microwave (CP-FTMW) spectrometer ${ }^{8,9}$. The frequency space of these instruments have ranged from radio frequencies to terahertz frequencies ${ }^{10-18}$, with many of the spectrometers requiring large chambers and rigorous vacuum systems as well as very time-consuming spectral acquisition. A popular design, the Balle-Flygare spectrometer ${ }^{19}$, can only measure a couple hundred $\mathrm{MHz}$ in an hour ${ }^{11}$. Because of this, rotational spectroscopy has spent most of its career as a purely laboratory based technique for fundamental physical chemistry laboratories and its analytical applications, although always present, have been very limited. The technique has been commercialized before ${ }^{20}$ without success, and it is expensive to build and develop instruments for this technique. An alternative to the expensive components that are normally used to build a spectrometer are monolithic microwave integrated circuits (MMICs). They are becoming more common place and low-cost, and advances in this technology are poised to transform microwave spectroscopy into a commercially viable and flexible technique. Widespread research and development in MMICs have been spurred by the increasing ubiquity of mobile communication, and the expanding economy of scale has driven prices on these general devices to all-time lows. Integration of these MMICs will significantly reduce the cost of modern day microwave spectrometers. The other 
advantage to using MMICs is that many of the available instruments are USB controlled and can be programmed easily through simple graphical user interfaces (GUIs) and application programming interfaces (APIs). Since the performance of the current electronics allows for true real-time acquisition, the ability to control the flow of incoming data in a fast and timely manner is a key factor to maximize the applications of these devices. In this chapter, a microwave spectrometer that has been built predominantly with MMIC components is presented. The sensitivity of the instrument is demonstrated, and the coherent measurement capabilities are shown. By using these techniques unknown molecules can be characterized in a complex mixture. With further development, this instrument could be a strong candidate for analytical chemistry applications.

\section{Experimental}

The schematic of the designed waveguide instrument is shown in Fig. 4.1. The descriptions of the pulse generation, receiver, sample chamber, and controlling software are below.

\section{a) Pulse generation}

In order to generate the single frequency excitation pulse and the local oscillator (LO) pulse (needed for the down conversion), an independent dual channel synthesizer 

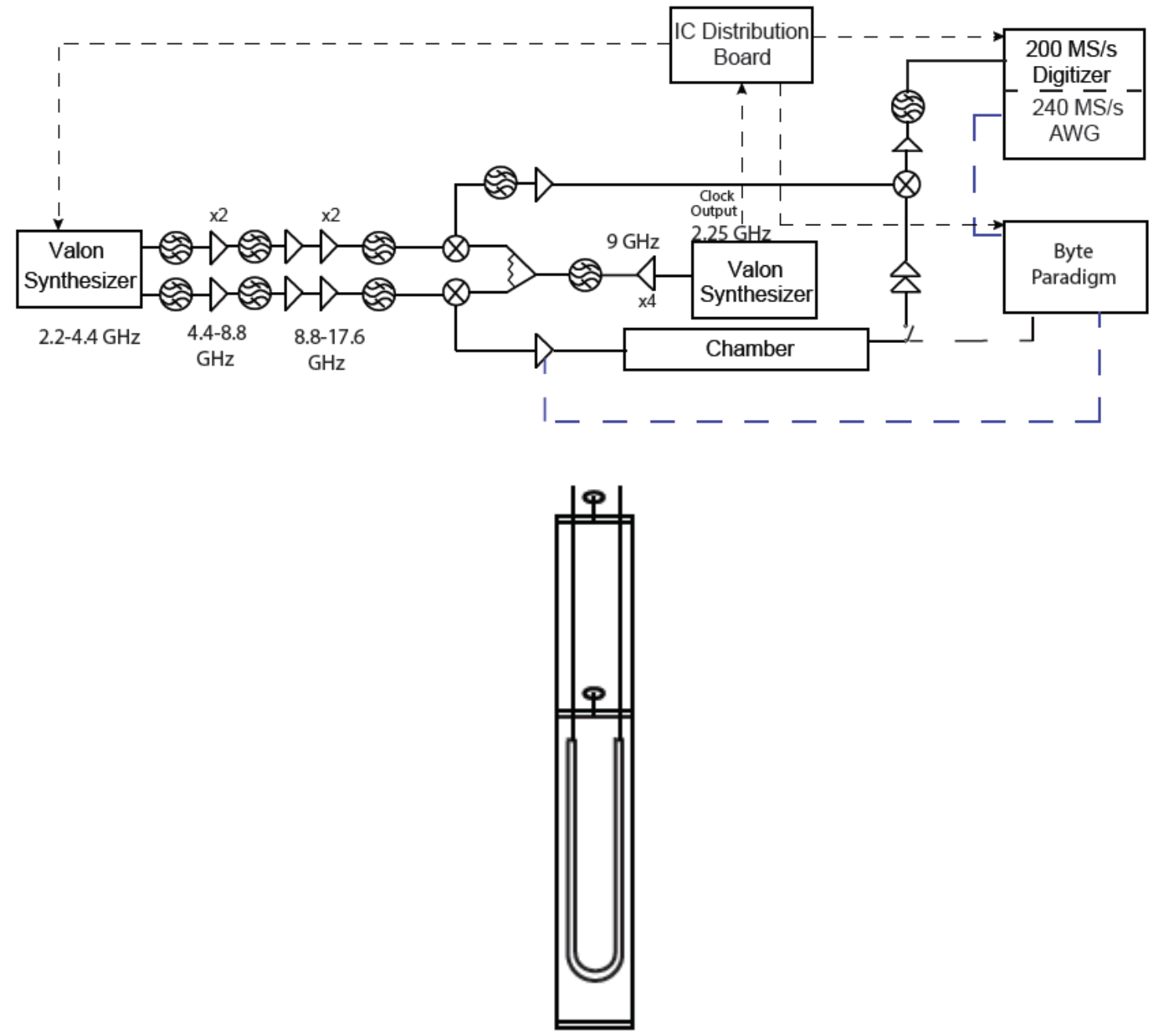

Figure 4.1. Schematic of the $18-26 \mathrm{GHz}$ waveguide instrument. The custom built chamber design can be seen below the schematic, in which the curved waveguide is in the active portion of the stainless steel chamber. 
(Valon 5008) was used. Each pulse was frequency multiplied by two doubling stages, filtered and amplified before finally being mixed up with a $9.0 \mathrm{GHz}$ frequency source. The up conversion 9.0 GHz frequency source was provided by a second Valon 5008 synthesizer with an output of $2.25 \mathrm{GHz}$. The output was then frequency quadrupled by a Marki active quadrupler (AQA1933K) and then split to up convert both the excitation pulse and the LO pulse. After the up conversion, the excitation pulse is amplified with a 2 Watt amplifier (Microwave Power L1826-34), and then attenuated by up to $27 \mathrm{~dB}$ by an ATM AF868-30 variable attenuator. If the full $2 \mathrm{~W}$ were used, the transitions became overdriven and signals were not seen. Two different sample chambers were used (see below). For the chamber design using microwave horns, this attenuation was varied. The pulse duration is changed depending on the molecular sample of interest so as to not drive the transition with more than a $\pi / 2$ pulse. The pulse duration is controlled with the TTL of the $2 \mathrm{~W}$ amplifier, which was driven with a 16 channel, $8 \mathrm{MB}$ pulse pattern generator (Byte Paradigm Wav Gen Xpress). The pulse pattern generator is set up so that 50 pulses are allowed to go through the system, followed by 50 experiments with no pulse. This allows for a background subtraction of the $10 \mathrm{MHz}$ spurious signals that are present from the various electronics. The overall timing of the instrument was set by the arbitrary waveform generator (AWG) on the Tiepie Handyscope HS5. The Tiepie features a $240 \mathrm{MS} / \mathrm{s}$ AWG as well as two $200 \mathrm{MS} / \mathrm{s}$ digitizer channels, each with a 32 Mpt memory depth. The external clock for the instrument is provided by the internal clock of the second Valon 5008. The output is set to $300 \mathrm{MHz}$ and fed to a divider board (Analog Devices AD9513). A $10 \mathrm{MHz}$ low voltage differential signal (LVDS) is sent to 
the Tiepie, a $10 \mathrm{MHz}$ CMOS signal is sent to the primary Valon, and a $100 \mathrm{MHz}$ CMOS signal is sent to the Byte Paradigm. This $100 \mathrm{MHz}$ clock gives the system a 10 ns pulse resolution, as the Tiepie only produces a signal at the beginning of each new cycle every $1 \mathrm{~ms}$.

\section{b) Detection}

After the sample chamber, the excitation pulse is discarded by a single pole double throw switch (Sierra Microwave SFD0526-001) so as not to damage or saturate the low noise amplifiers (LNA) needed to amplify the molecular free induction decay (FID). The LNA is a combination of two amplifiers, a Hittite HMC504LC4B and a WiseWave AHP-21032735-01 amplifier. The Hittite amplifer sets the noise floor to $2.2 \mathrm{~dB}$ and has a gain of $19.5 \mathrm{~dB}$, while the Wisewave amplifier has a gain of $35 \mathrm{~dB}$. After amplification, the molecular signal is mixed down with a Marki Microwave mixer (M1R-0726L). The LO, for the mixer, was amplified by a Hittite HMC504LC4B amplifier. The downconverted signal is digitized on one of the digitizer channels of the Tiepie. The overall dimensions of the electrical portion of the instrument are 81 x 61 x $10 \mathrm{~cm}$.

\section{c) Sample chamber}

Two sample chambers were used. The first was a custom built static waveguide chamber which is described first. The second was a static cell, horn broadcast and receiver setup.

The sample chamber has been designed to create a static environment over long periods of time ( 2 months). The $50 \mathrm{~cm}$ WR42 waveguide was placed in a stainless steel tube 
(20" length, 6-3/4" diameter) and sealed with copper gaskets. The total volume of the tube is approximately $5 \mathrm{~L}$. Since the pulses and molecularFID need to be coupled into and out of the chamber, it was imperative to consider the leak rates of both the gas sample introduction method and the feedthroughs for the pulses in and out of the chamber. SMA feedthroughs were placed together on one of the end flanges, along with a Swagelok B-series stainless steel valve (SS-4BW). The leak rates were $1.32 \times 10^{-8}$ Torr* $\mathrm{L} \mathrm{s}^{-1}$ and $5 \times 10^{-9}$ Torr* $\mathrm{L} \mathrm{s}^{-1}$ for the feedthroughs and valves respectively (leak rates were obtained from the companies). The chamber was pumped out through the valve by a Varian fan cooled diffusion pump (AX65) and a 1402 DuoSeal Welsh mechanical pump. In order to reduce the leak rates that occur at the SMA feedthroughs and the value, a second stainless steel tube (10" length) was used as a "buffer zone”. An example of the chamber can be seen in Fig. 4.1. Once the active chamber was prepared and sealed off, the secondary chamber was pumped out using the same pump system, and then closed off using another B-series valve.

For the second setup, a stainless steel 6-way cross with ISO 200 ports was used. The chamber was sealed off at the horns by using Kapton waveguide windows (ATM 42-233-6-8). The $25 \mathrm{~dB}$ gain horns (ATM 42-443-6) were placed $12.5 \mathrm{~cm}$ away from each other. That coupled with the length of the horns gave a total length of $60.5 \mathrm{~cm}$ that the pulse traversed. The total volume of sample that the pulse interacted with is $3260 \mathrm{~cm}^{3}$. The chamber was pumped out by an Agilent TPS-compact turbo pump. The pressure was measured using a 50 mTorr MKS Baratron manometer (627B-22683). 
Samples of liquefied anhydrous ammonia from Matheson, $\geq 97.5 \%$ carbonyl sulfide and 99\% nitrous oxide from Sigma Aldrich, and reagent grade methanol from Fischer Scientific were obtained and used without any further purification.

\section{d) Graphical user interface}

The graphical user interface (GUI) shown in Fig. 4.2 was designed with the museum exhibit it will be a part of in mind. It was modified to perform research grade experiments, such as nutation and detuning experiments as well. The development was done on an Intel NUC (D54250WYK) running Windows 7 and 32-bit Python 2.7. The GUI was written with the aid of the PyQt module. It was used to control the digitizer, the Valon synthesizers, and the Byte Paradigm, as well as display the data. For the data workup, the FFT function from the numpy module was used. In the displayed GUI, the user selects a push button for the desired molecule. The instrument changes the frequency and the pulse duration to that of the selected molecule. The data is acquired, FTed and displayed in a running average. For this implementation, information about each molecule is shown along the left hand side, along with facts about its discovery in the interstellar medium. Below the live data, a graph of the transitions from the Robert C. Byrd Green Bank Telescope (GBT) PRebiotic Interstellar MOlecule Survey (PRIMOS) ${ }^{21}$ data is shown for comparison. 


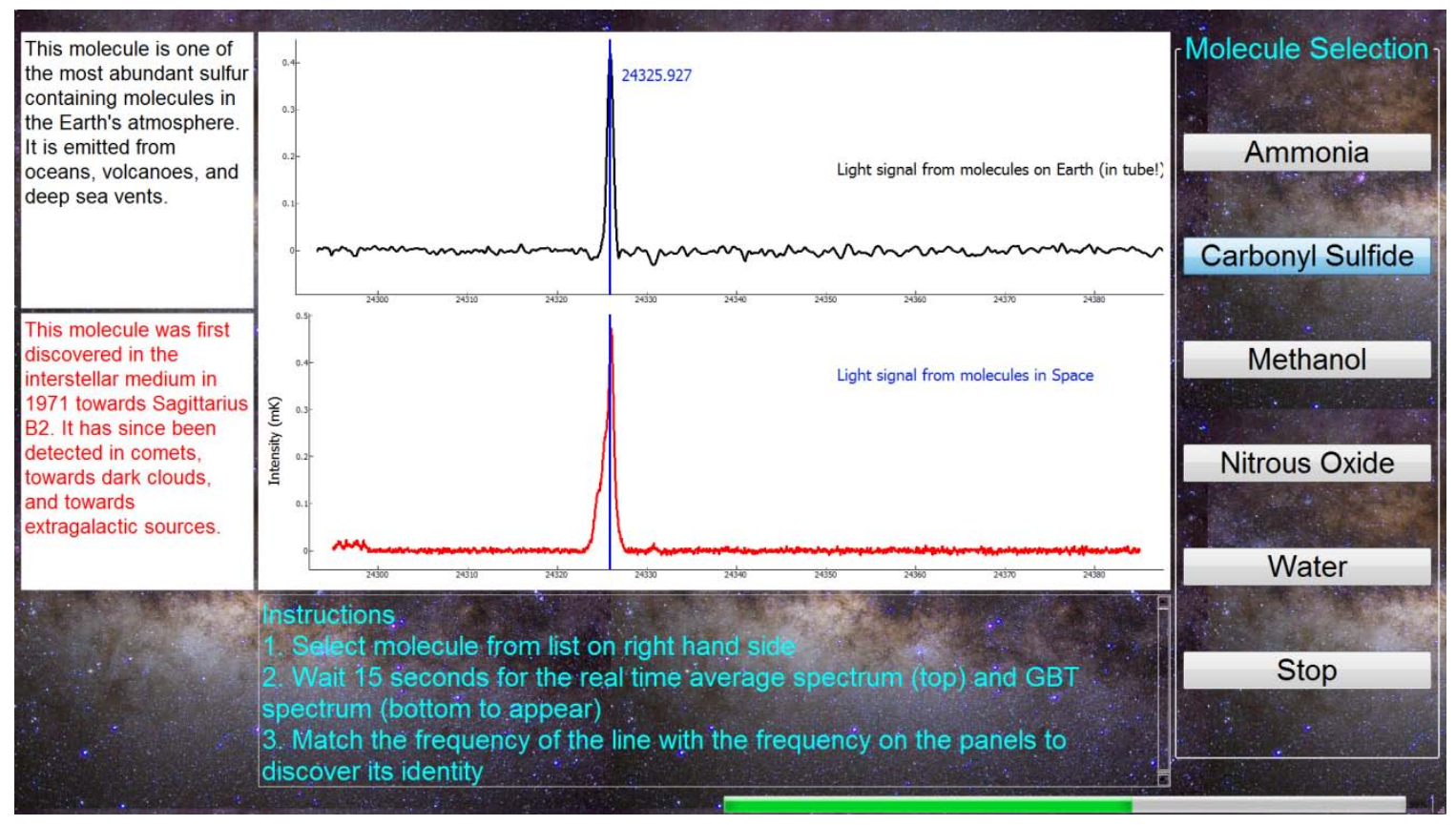

Figure 4.2. The GUI that was developed for the control of the instrument as well as the data workup for the museum exhibit. The user selects the push button for the molecule that they would like to study on the right. Information about the selected molecule is displayed on the left; the terrestrial information is shown in the top box and the interstellar information is displayed in the bottom. The top panel displays the running average of the molecular emission (in this case OCS) in the chamber, and it is compared to the astronomical PRIMOS dataset at the same frequency. 


\section{Results}

The phase stability of the instrument is demonstrated in Fig. 4.3. The top panel shows a single acquisition of the spectrometer. Effectively 100 "experiments" are

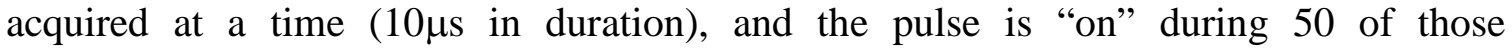
experiments and "off" during the second half. This allows for an electrical background subtraction of any spurious signals that are present in the spectrum. In the bottom panel 100 acquisitions of the described experiment were added together to produce an average FID that incorporates 5000 FIDs. This demonstrates the phase stability of the experiment.

For one of the benchmark measurements, the $3_{3^{+}}-3_{3-}$ transition of ammonia was measured. The pulse duration was optimized to give the best signal-to-noise ratio (SNR) and the measured signal is displayed in Fig. 4.4. As can be seen from the figure, the signal intensity is equivalent between the left and right panel, but the noise drops by a factor of 10 after 100 averages. The SNR for the single shot spectrum is 100:1.

The ability of this instrument to perform measurements that could be used to characterize unknown molecules is shown in Fig. 4.5. The nutation curve for three different molecules is shown on the left panel. The three molecules chosen for this experiment were ammonia, methanol, and OCS. Each is different in size, dipole moment,

and symmetry. In the nutation experiment, the pulse duration was increased by 10 ns steps from a starting duration of $70 \mathrm{~ns}$. The signal intensity was monitored as a function 

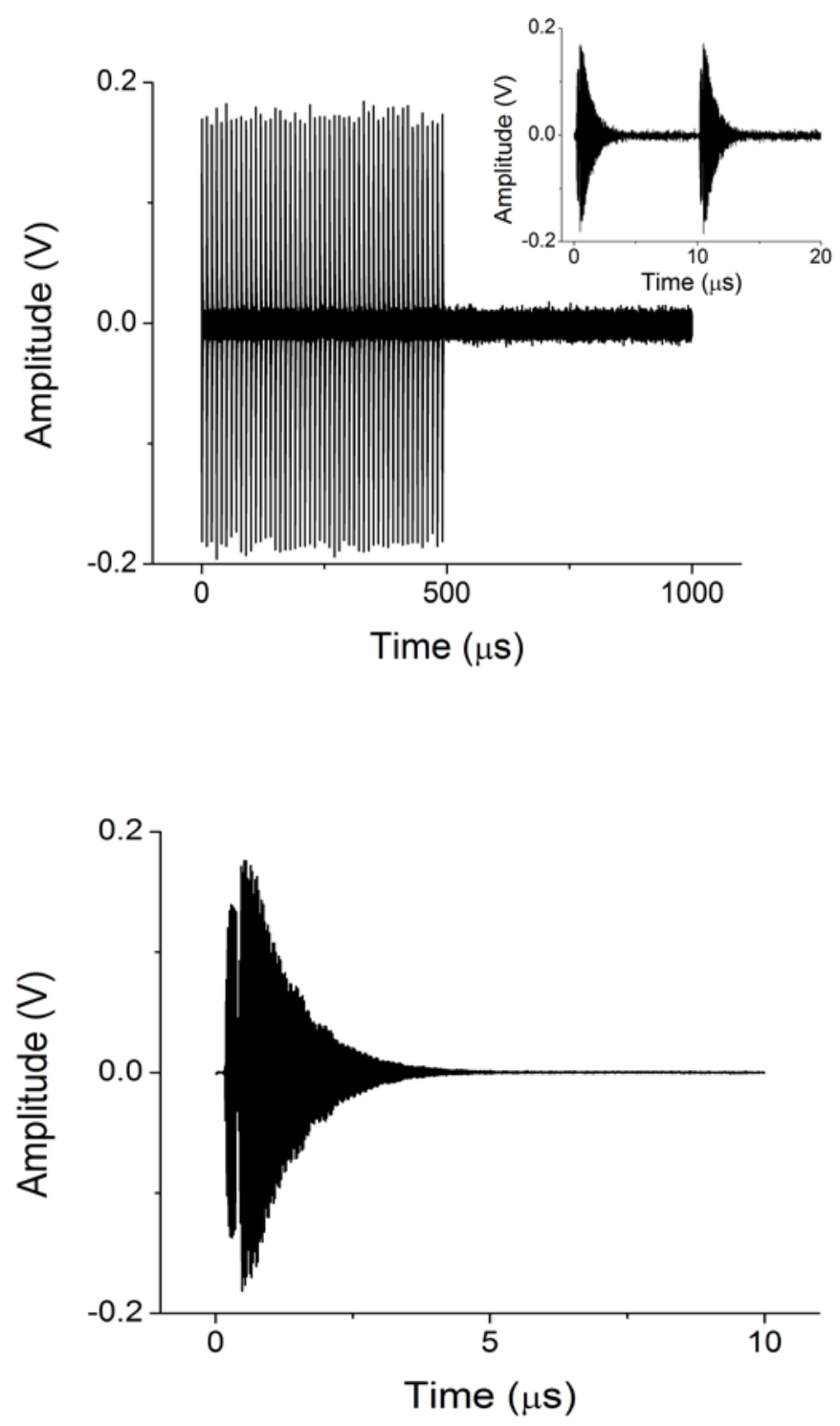

Figure 4.3. Shown in the top panel is a single time domain acquisition of the instrument. Over the course of $1 \mathrm{~ms}$, one hundred $10 \mu$ s experiments are acquired. The pulse is not "turned on" for 50 of the experiments so that a background subtraction can be made. The overall amplitude of the FID for the $3_{3_{+}}-3_{3-}$ transition of ammonia is $170 \mathrm{mV}$ at 7 mTorr. The bottom panel shows the FID strength after 5000 experiments have been averaged together in the time domain. The FID strength did not change, showing that the independent acquisitions are phase coherent (to within $10 \mathrm{~ns}$ ) from one dataset to the next. 


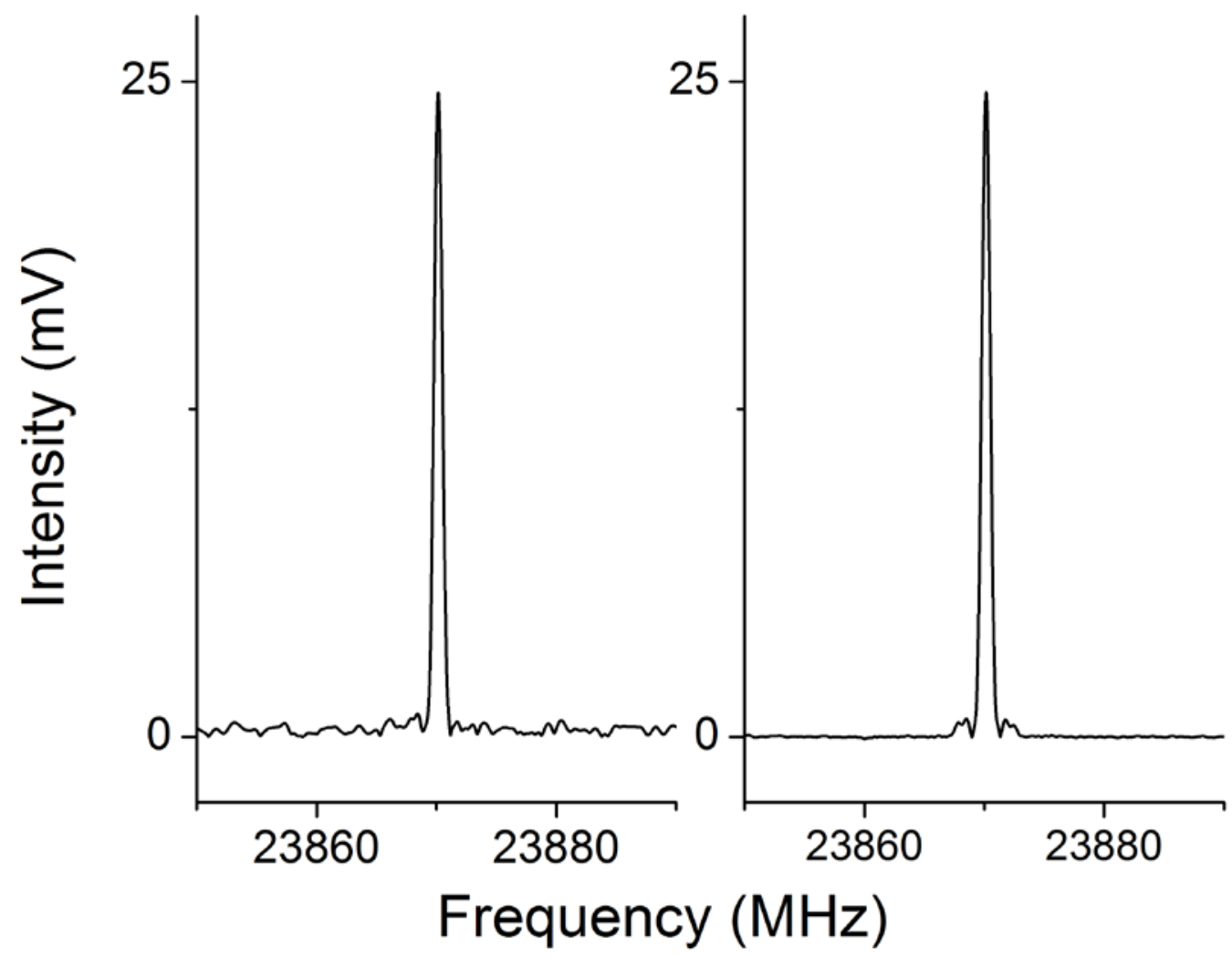

Figure 4.4. The left panel shows the $3_{3^{+}}-3_{3-}$ transition of ammonia after one acquisition, measured at a pressure of $7 \mathrm{mTorr}$. Displayed on the right is the same transition after 100 averages. The rms noise floor fell from $0.25 \mathrm{mV}$ to $0.025 \mathrm{mV}$. 


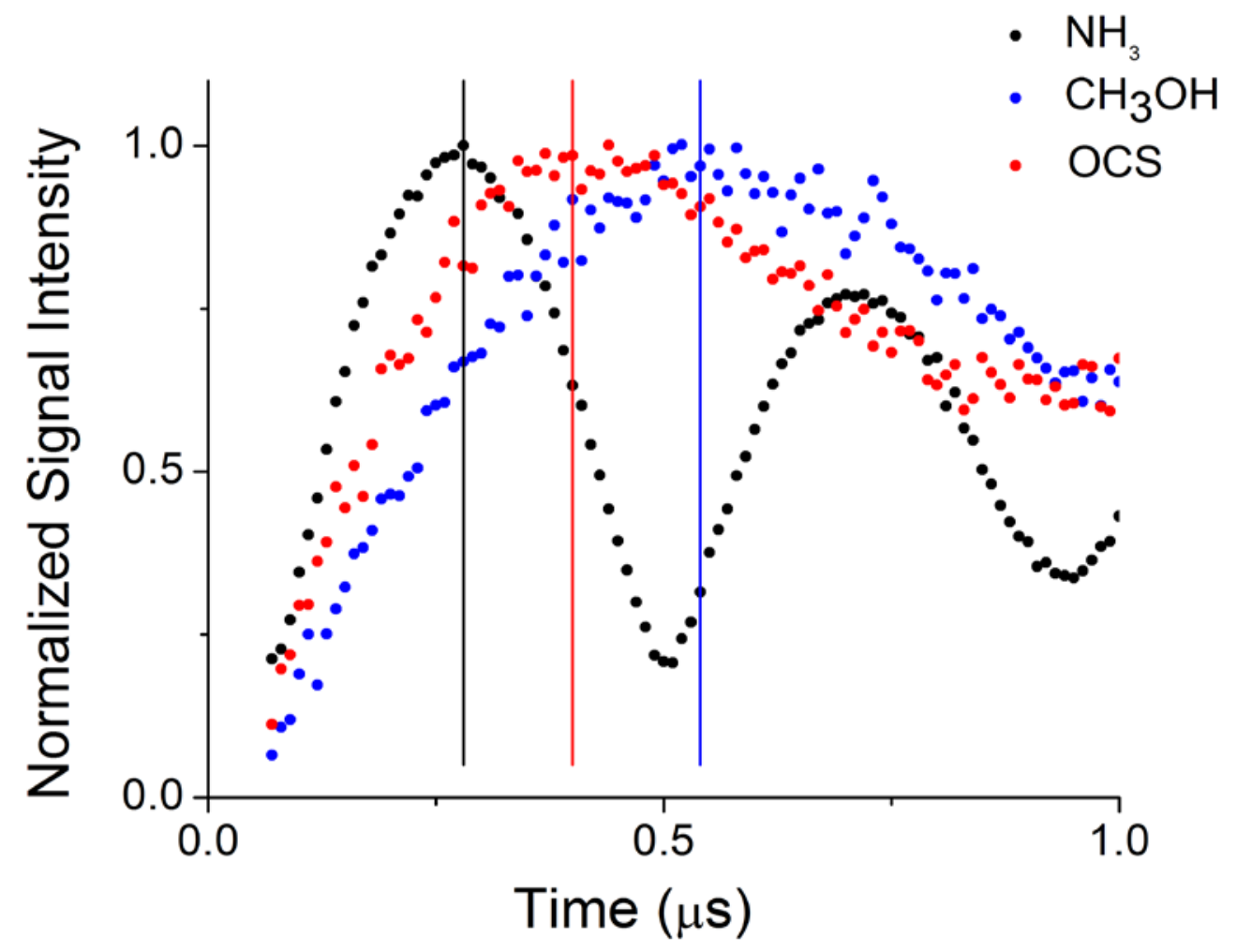

Figure 4.5. The nutation curves of ammonia (black), methanol (blue), and OCS (red) are shown. A single acquisition for ammonia and OCS and 50 acquisitions for methanol were recorded. The signal intensities were normalized to 1 . The $\pi / 2$ pulse duration was found to be $280 \mathrm{~ns}, 400 \mathrm{~ns}$, and $540 \mathrm{~ns}$ for ammonia, methanol, and OCS respectively, pressures of 7 mTorr, 10 mTorr, and 24 mTorr. The ammonia nutation curve distinctly shows the pulse duration of the $\pi$ pulse and rebounds into the second part of the Rabi cycle. 
of time, allowing us to find the time of maximum signal intensity. This pulse duration corresponds to the $\pi / 2$ pulse for that molecule in a constant electric field. The $\pi / 2$ pulse durations were found to be $280 \mathrm{~ns}$ for ammonia, $400 \mathrm{~ns}$ for methanol, and 540ns for OCS. The nature of the nutation experiment allows for a characterization of the electric field, if the transition dipole moment of the molecule is known, or for a characterization of the transition dipole moment, if the electric field is known, as shown in Eq. $4.1^{22}$

$$
\omega_{R}=\frac{M_{10} E}{\hbar}
$$

where $\omega_{\mathrm{R}}$ is the Rabi frequency in $\mathrm{Hz}, \mathrm{M}_{10}$ is the transition dipole moment in $\mathrm{C} * \mathrm{~m}, \mathrm{E}$ is the electric field in $\mathrm{V} / \mathrm{m}$, and $\hbar$ is Planck's constant. For this instrument the electric field was calculated to be $2.6 \mathrm{~V} / \mathrm{cm}$ from Eq. 4.2 which was presented by Ekkers and Flygare $^{23}$ :

$$
P=E_{0}^{2}\left(\frac{a * b}{480 * \pi}\right) \frac{\lambda}{\lambda_{g}}
$$

In which $\mathrm{P}$ is the power in watts, $\mathrm{E}_{0}$ is the maximum field amplitude in $\mathrm{V} / \mathrm{cm}$, $\mathrm{a}$ and $\mathrm{b}$ are the length and width of the waveguide slit, $\lambda$ is the freespace wavelength and $\lambda_{\mathrm{g}}$ is the waveguide wavelength. The power was determined at each molecular frequency and the electric field for the $\mathrm{J}=2-1$ transition of OCS at $24325.927 \mathrm{MHz}$ and the $3_{3_{+}}-3_{3-}$ transition of ammonia at $23870.13 \mathrm{MHz}$ was found to be $2.6 \mathrm{~V} / \mathrm{cm}$, while it was $1.8 \mathrm{~V} / \mathrm{cm}$ for methanol. Knowing this, the transition dipole moments can now be back-calculated using Eq. 4.1. 
Fig. 4.6 shows the detuning experiments performed using ammonia. Once the $\pi / 2$ pulse duration is determined, a detuning curve can be used to look at the signal intensity as a function of pulse duration. In order to scan over bandwidths of $20 \mathrm{MHz}$, a pulse duration of 50 ns must be used. For ammonia, this would not be a problem as it has high signal intensity under optimal conditions, but for something that is weak, lowering the power spectral density could be detrimental. For the measurement, the pulse duration was set to the $\pi / 2$ duration, $280 \mathrm{~ns}$. The pulse frequency was set $12 \mathrm{MHz}$ off resonance of the line center and stepped $400 \mathrm{kHz}$ to sweep over resonance and continue to $12 \mathrm{MHz}$ above resonance. The resulting figure is a sinc function that has a FWHM of approximately 3.57 MHz. A second detuning curve was run in the weak pulse limit with a pulse duration of 200 ns. It can be seen that the power spectral density decreases and the FWHM of the detuning curve is now $5 \mathrm{MHz}$. This is important when setting up the parameters for a frequency scan because if large frequency steps are desired, the detuning curve can indicate the signal intensity of the transition measured at the desired frequency bandwidth.

The instrument has the capability of acquiring a broadband spectrum. A scan mode has been developed in which the frequency step is dependent on the FWHM of the pulse. For Fig. 4.7, a 100 average spectrum of $1.4 \mathrm{GHz}$ of ammonia was measured with 50 ns pulses, to give steps of $20 \mathrm{MHz}$. The spectrum intensities were corrected for the intensity roll off of the pulse. The intensity accuracy can be seen in Fig. 4.7 in which the experimental spectrum is plotted against the simulated spectrum ${ }^{24}$. The signal 


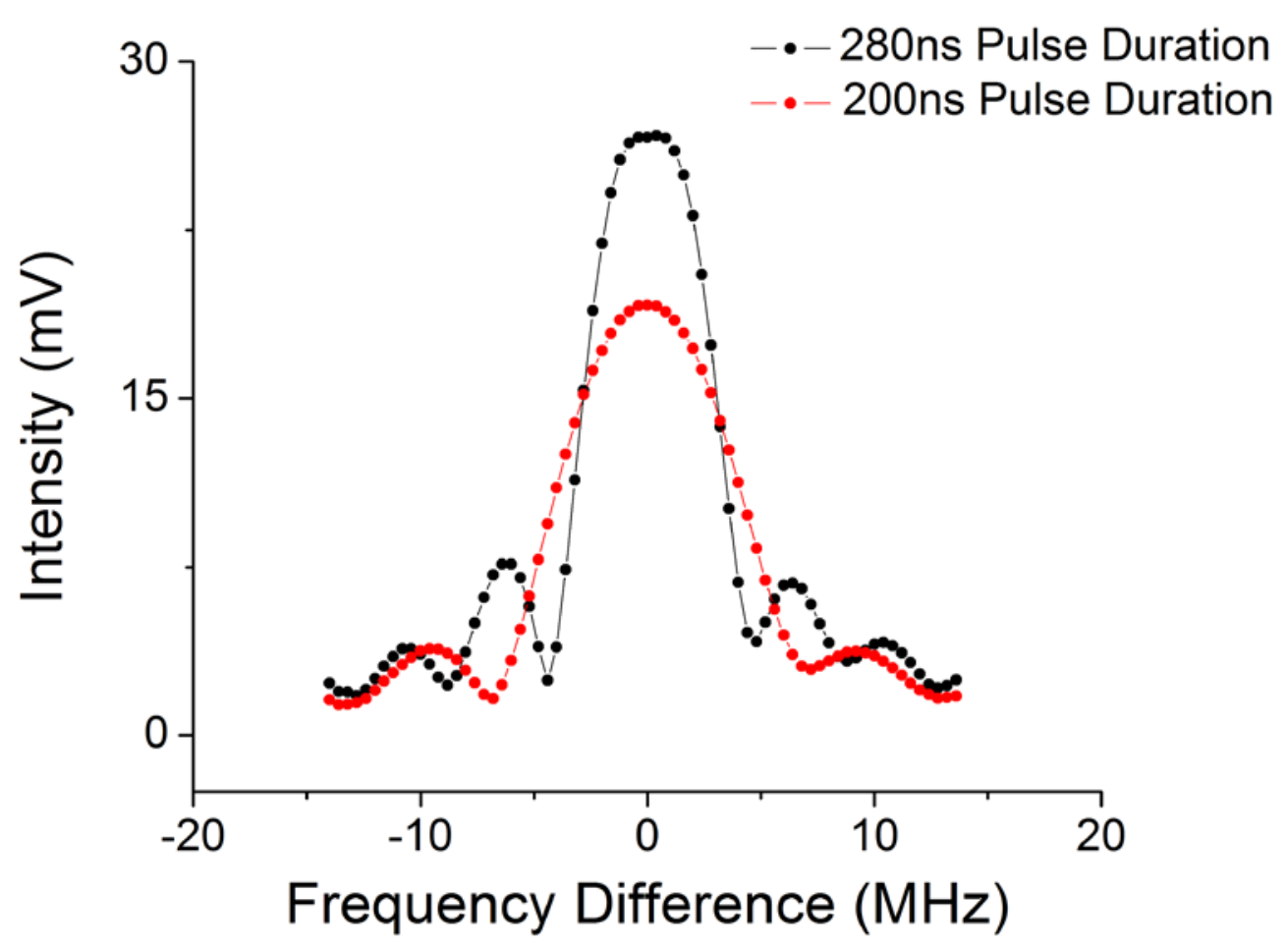

Figure 4.6. The detuning curve for ammonia is shown at a pulse duration of $280 \mathrm{~ns}$ and 200 ns. The scan was started $12 \mathrm{MHz}$ off resonance and moved over resonance to 12 $\mathrm{MHz}$ past resonance. The FWHM for the $280 \mathrm{~ns}$ pulse duration is $3.57 \mathrm{MHz}$ while it is 5 $\mathrm{MHz}$ for the 200 ns pulse. 


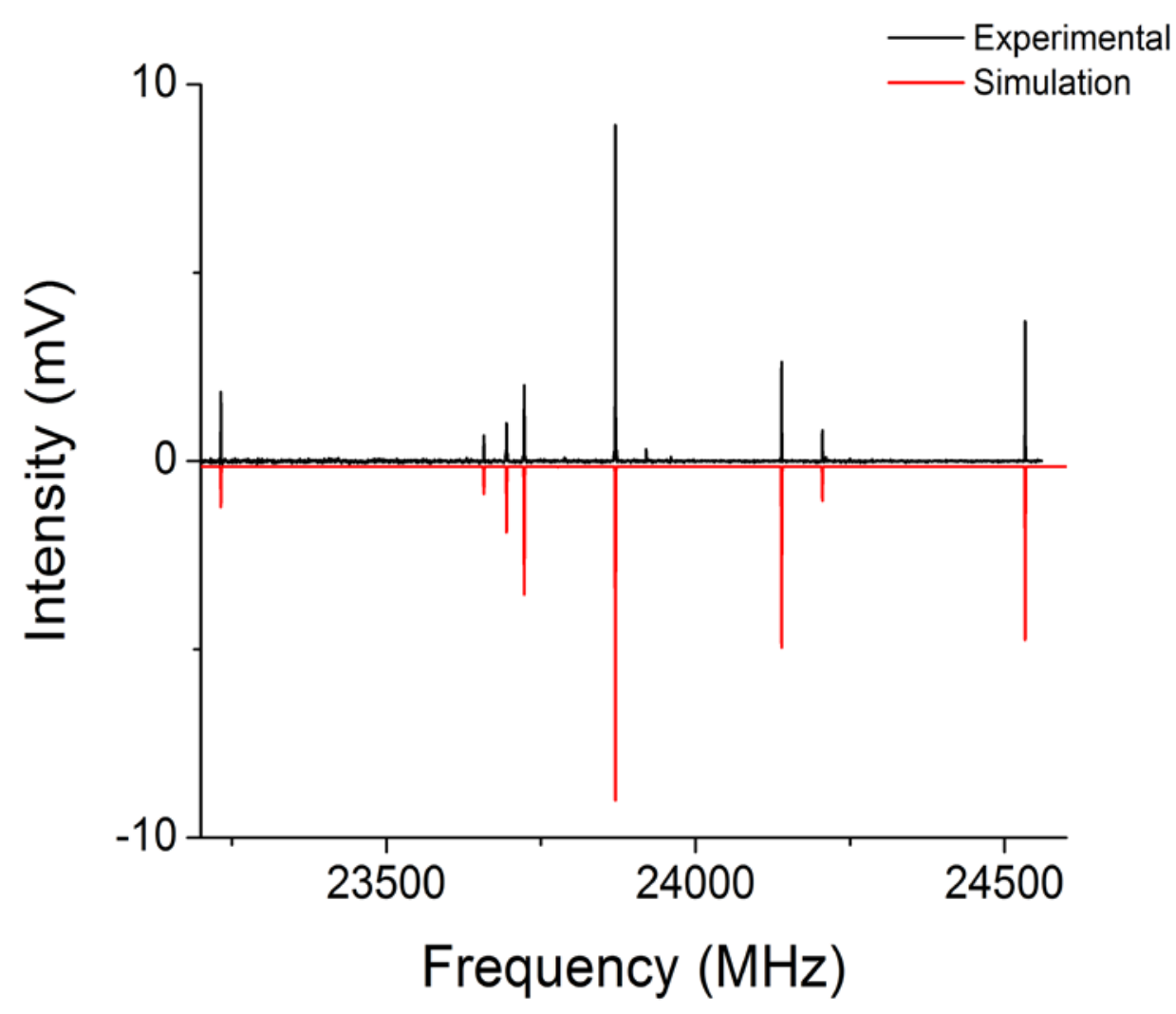

Figure 4.7. A 100 average $1.4 \mathrm{GHz}$ scan of $7 \mathrm{mT}$ Torr of ammonia. The black trace is the experimental spectrum and the red trace is the simulation made with the .cat file from $\mathrm{JPL}^{24}$. A $50 \mathrm{~ns}$ pulse duration (FWHM of $20 \mathrm{MHz}$ ) was used, so that $20 \mathrm{MHz}$ of bandwidth could be covered at a time. The maximum signal intensity was $8.9 \mathrm{mV}$. 
intensity, which falls as the square-root of the ratio of pulse bandwidths, is $8.9 \mathrm{mV}$. When comparing the two setups, the scan mode and the single frequency measurement, the pulse duration decreased from $280 \mathrm{~ns}$ in the single frequency mode to $50 \mathrm{~ns}$. This gives a pulse duration ratio of 5.6 and a scaling factor of $\sim 2.3$. From this it is expected that the signal level would be $10.5 \mathrm{mV}$ as opposed to $25 \mathrm{mV}$ seen in the single frequency measurement. The discrepancies in signal intensity could be arising from the fact that the sample waveguide is in a stainless steel chamber and the ammonia will react with the chamber over a short period of time.

Besides demonstrating these techniques, super-radiance was tested with measurements in the waveguide and a horn broadcast and receiver system. Panel $\mathrm{A}$ in Fig. 4.8 shows the signal level for ammonia at 7 mTorr in the waveguide after 100 averages. The signal intensity is $25 \mathrm{mV}$. This is for a power of $1.21 \mathrm{dBm}$ and a total volume of $23 \mathrm{~cm}^{3}$. The microwave circuit was left in the same configuration, but this time it was broadcast across the static gas horn setup. The distance the between the horns from end to end was $60.5 \mathrm{~cm}$, and the total molecular interaction volume was $3262 \mathrm{~cm}^{3}$. The signal level increased to $50 \mathrm{mV}$. The power was then increased by a factor of 50 , and the signal increased in the horn setup to $200 \mathrm{mV}$ as shown in panel C. 


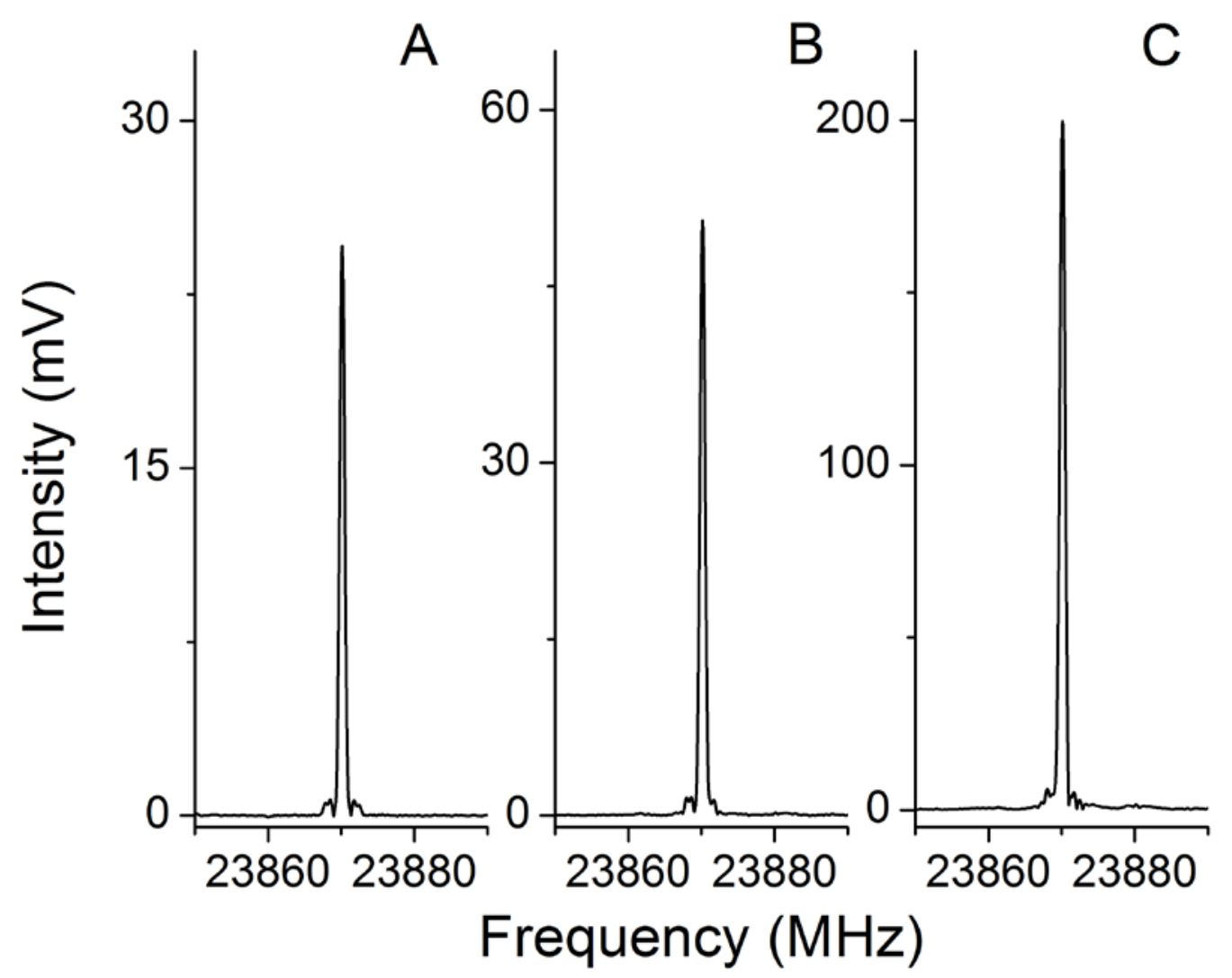

Figure 4.8. This figure shows the effects of population verses power. Panel A shows the optimized signal level for ammonia within the waveguide spectrometer. The signal intensity is $25 \mathrm{mV}$ with $1.2 \mathrm{~dB}$ of power going into the chamber. In panel $\mathrm{B}$, the same electrical circuit and power level was used, but this time the experiment was taken with the horn broadcast/receiver setup. The signal level was $50 \mathrm{mV}$. The pulse duration used for both panel A and B was 280 ns correlating to the pulse duration determined by the nutation curve in the waveguide setup. By adding 250 times more power, the $\pi / 2$ pulse duration was found to be 170 ns. With this pulse duration, the signal increased to 200 $\mathrm{mV}$. All measurements were taken at 7 mTorr and 100 averages. 


\section{Discussion}

The instrument described above is a low cost alternative to conventional microwave spectrometers. While it does not have the broadband capabilities of CPFTMW, it does have the advantage of lower sample consumption and the capabilities to perform characterization measurements so that it could be implemented as an analytical instrument. The static cell design allows for samples to be repeatedly measured so that trace sample detections can be made. The instrument was designed with the final destination of a museum exhibit in mind. Thus, the sample chamber was created so that it could hold vacuum for up to 2 months with only a 33\% decrease in the original signal levels, even though this is more stringent that what would likely be needed for an analytical instrument.

For the instrumentation, the large memory depth of the Tiepie, both for the digitizer and AWG, allow large datasets to be acquired. This report demonstrates 100 experiments being acquired in a single acquisition, shown in Fig. 4.3. However, with the 32 Mpt memory depth on the Tiepie digitizer a maximum of 16,000 experiments could be collected in one acquisition. Although the transfer times to the computer increase by a factor of 100 from a 20,000 pt (100 averages) collection to a 2,000,000 pt (10,000 average) collection, the advantage to collecting a larger amount of data is that the sample conditions within the cell will drift less for the 10,000 average acquisition than for a sequence of 100 acquisitions of 100 averages. The internal clock of the Valon 5008 is stable enough to be able to average coherent emission for more than 100,000 averages, 
and the baseline noise level of the spectrometer falls as expected with averaging. This is demonstrated in Fig. 4.4 and the noise level has been monitored up to 100,000 averages. The signal levels in Fig. 4.4 can also be used to calculate the detection limit of ammonia. The calculated detection limit of a 7 mTorr sample of ammonia is roughly 2 ppm in 10,000 averages, acquired in less than a minute. This calculation is done by scaling the signal level obtained to a 3:1 SNR and determining the pressure that would be needed. It is then assumed that the chamber can be pressurized to 100 mTorr using $\mathrm{N}_{2}$. This could be enhanced by pairing this spectrometer with a real-time digitizer in which a detection limit of $700 \mathrm{ppb}$ can be acquired in about 1 minute. While not comparable to other commercial instruments such as the cavity ring down spectrometer by Picarro ${ }^{25}$ which as a detection limits of ppb, this instrument is an inexpensive alternative that offers a way to not only monitor a target sample but also to quickly assess the optimal conditions in which to monitor it.

The ability of this instrument to help determine the molecular identity of species within unknown samples hinges on its capabilities to perform measurements such as the nutation experiment. The nutation experiment exploits the relationship between the dipole moment and electric field to determine the Rabi frequency of the two level system in question. In a two level system a $\pi / 2$ pulse duration brings the system into its super-position state, giving the highest signal level. Fig. 4.5 shows the nutation curves for ammonia, methanol, and OCS. Ammonia has the largest transition dipole moment, and thus it would be expected to have the shortest pulse duration from Eq. 4.1. If all things 
were equal, it would be followed by methanol and finally by OCS. With an electric field of approximately $2.6 \mathrm{~V} / \mathrm{cm}$, the optimal pulse duration for OCS and ammonia was $540 \mathrm{~ns}$ and 280 ns respectively. The electric field at the frequency of the methanol transition was $1.8 \mathrm{~V} / \mathrm{cm}$, yielding a $\pi / 2$ pulse duration of $400 \mathrm{~ns}$. The difference in the electric field is due to some of the filters that were used in the spectrometer, and the spectrometer could be modified so that the power is equal across all of the frequencies. By characterizing the electric field across the entire band of the spectrometer, one could use this information and determine the transition dipole moment for an unknown molecule. As an example, the transition moments for ammonia were calculated to be $0.738,0.738,0.651$, and 0.426 Debye for each of the $\mathrm{M}$ components ${ }^{26}$ and an average nutation curve of the $4 \mathrm{M}$ components was simulated to have a $\pi / 2$ pulse duration of 280 ns at an electric field of 2.6 V/cm. By pairing this with a broadband scan and potentially double resonance if needed, the molecule's identity could then be determined.

Because we are using a single frequency source and not a chirped pulse instrument, the bandwidth of the pulse is inversely proportional to the pulse duration. By exploiting this feature of a square wave pulse, wideband measurements can be obtained. The detuning curve in Fig. 4.6 demonstrates this idea. As the excitation frequency of the pulse is moved closer to resonance, the signal intensity of the $3_{3_{+}}-3_{3_{-}}$transition of ammonia becomes stronger, until resonance is reached. At this point, optimal excitation is achieved. The excitation frequency of the pulse is then moved off resonance again, and the maximum signal intensity drops the further away from resonance the excitation pulse 
is. The result is a sinc function, as is expected in a detuning experiment ${ }^{4}$. The top of the sinc flattens out due to the fact that the system is being overdriven. A 280 ns pulse duration drives the system slightly past $\pi / 2$; however with the resolution of the spectrometer, only 10 ns increments can be used for the pulse duration. For a measurement in the weak pulse limit, such as the 200 ns pulse duration measurement, this is not the case and a perfect sinc can be seen.

A scan of the ammonia spectrum was desired; however, it can be seen that for a pulse duration of 280 ns approximately $3.57 \mathrm{MHz}$ could be covered at once. For a 1.4 GHz spectrum, this would require almost 400 measurements. If the pulse duration is shortened to $200 \mathrm{~ns}$ the maximum frequency coverage increases to $5 \mathrm{MHz}$, decreasing the number of measurements to 280. The drawback of this practice is that the maximum signal intensity decreases as the power spectral density decreases. By shortening the pulse duration by 80 ns (28\% of the optimum) the peak intensity also dropped by $56 \%$. Thus for a weak species, a targeted search would be required. Using this inverse relationship between pulse duration and bandwidth, a scan of ammonia was taken with a 50 ns pulse duration and is displayed in Fig. 4.7. The simulated spectrum of ammonia is displayed across the bottom. The experimental spectrum, shown in black, was intensity corrected for the fact that the power is not equal across the measured bandwidth. The power is distributed across a sinc function, as is mimicked in the detuning measurements. There is good agreement between the JPL simulated spectrum and the experimental spectrum. A decrease of $61 \%$ was seen in the maximum intensity of the $3_{3+}-3_{3-}$ 
transition. While this was still more than adequate for the spectrum of ammonia to be measured, such a decrease in power would result in longer scanning times for molecules such as $\mathrm{N}_{2} \mathrm{O}$, which can be seen at $16: 1$ on 100 averages. When measuring samples that are time sensitive, such as the emission of several compounds from soil, fast measurement times are essential. The ability to be able to control the optimal power level could play a key role in the use of this instrument. This instrumental design presented here has the capability to output $2 \mathrm{~W}$ of power; however, for most molecules this was too much power and would overdrive the molecule beyond its $\pi / 2$ pulse.

The idea of super-radiance was tested using both the waveguide and the broadcast/receiver system. The results shown in Fig. 4.8 demonstrate the effect of using the optimal power per unit area versus having a large volume of gas, but less power per unit area. Panel A of Fig. 4.8 shows the signal intensity of ammonia in the waveguide instrument. The pulse duration was set to 280 ns, which was determined by the nutation curve. The signal level was $25 \mathrm{mV}$. By moving to the broadcast/receiver system, the volume increased by a factor of $\sim 140$. The same power level and pulse duration were used, and a signal level of $50 \mathrm{mV}$ was measured for this setup in the same configuration as the waveguide. Even though the power level per unit area was lower in the broadcast/receiver system, the super-radiance of more molecules in the system outweighed the power disadvantage to give a higher signal level. To further investigate this phenomenon, attenuation from the system was removed. In order to obtain the same electric field in the spectrometer, 140 times more power would have been needed to 
achieve a $280 \mathrm{~ns} \pi / 2$ pulse duration. However, 250 times more power was chosen to be added to the system due to the sensitivity of the variable attenuator. The nutation curve revealed the $\pi / 2$ pulse duration to be $170 \mathrm{~ns}$, consistent with the fact that 1.7 times more power was added to the system than the needed factor of 140 . Using this $\pi / 2$ pulse, the measurement in panel $\mathrm{C}$ of Fig. 4.8 was obtained. The maximum signal intensity increased to $200 \mathrm{mV}$. With the use of 250 times more power, it was expected that the signal intensity would increase by a factor of approximately 16 according to Dicke ${ }^{7}$. However, we only witnessed a factor of 8 gain in the intensity. It may have been that the horns were not properly aligned within the chamber, even though care was taken to do so. From the nutation data, we can be confident that the correct power is in the chamber, thus ruling out a power fluctuation from the attenuator. The idea of super-radiance has been investigated and with it the gain that a broadcast/receiver system has over the waveguide system, provided that sufficient power is available. However, more testing needs to be done in order to determine the exact effects that are being witnessed in the system. Effects such as those from changes in the excitation beam shape could be an important factor. While the waveguide is more compact, it can be quite costly to increase the path length, and holding it at constant pressures for significant periods of time is quite difficult. 


\section{Conclusions}

This chapter has shown the development of low cost $(<\$ 20,000) 18-26 \mathrm{GHz}$ spectrometer. Most instruments with the stand alone AWG and digitizer require large amounts of power, time, and can be difficult to program so that the data flow is continuous both to the instrument and to the computer. As more MMIC components become available and the move towards USB capabilities (especially USB 3.0) more complete, instruments, such as the one presented in this paper, will become more common place. This instrument offers not only a standalone option for chemical monitoring and analysis, but when paired with an existing instrument, it could become an invaluable tool for determining optimal experimental conditions to monitor a variety of species. The ability to complete a suite of measurements such as a scan, nutation curve, detuning curve, and single frequency measurements opens up the possibilities to new uses. The idea of super-radiance has been tested. With this demonstration comes the ability to design instruments such that they fit the user's needs. The robustness and cost effectiveness of this instrument will also allow it to be placed in such environments as a high school chemistry laboratory or a physical chemistry laboratory at the university level, making rotational spectroscopy and its capabilities more common place. 


\section{Chapter 4 References}

(1) Brewer, R. G.; Shoemaker, R. L. Phys. Rev. Lett. 1971, 27, 631-634.

(2) Hocker, G. B.; Tang, C. L. Phys. Rev. 1969, 184, 356-359.

(3) Harris, B. J. A Chirped Pulse Fourier Transform Millimeter Wave Spectrometer for Room Temperature, Gas Mixture Analysis. Doctor of Philosophy, University of Virginia, 2014.

(4) Kaufman, A. M.; Lester, B. J.; Regal, C. A. Phys. Rev. X 2012, 2, 041014.

(5) Iii, R. C. W.; Ronn, A. M.; Jr, E. B. W. Rev. Sci. Instrum. 1966, 37, 927-933.

(6) Wodarczyk, F. J.; Wilson, E. B. J. Mol. Spectrosc. 1971, 37, 445-463.

(7) Dicke, R. H. Phys. Rev. 1954, 93, 99-110.

(8) Brown, G. G.; Dian, B. C.; Douglass, K. O.; Geyer, S. M.; Pate, B. H. J. Mol. Spectrosc. 2006, 238, 200-212.

(9) Brown, G. G.; Dian, B. C.; Douglass, K. O.; Geyer, S. M.; Shipman, S. T.; Pate, B. H. Rev. Sci. Instrum. 2008, 79, 053103.

(10) Pérez, C.; Lobsiger, S.; Seifert, N. A.; Zaleski, D. P.; Temelso, B.; Shields, G. C.; Kisiel, Z.; Pate, B. H. Chem. Phys. Lett. 2013, 571, 1-15.

(11) Grabow, J.-U.; Stahl, W.; Dreizler, H. Rev. Sci. Instrum. 1996, 67, 4072-4084.

(12) Serafin, M. M.; Peebles, S. A. J. Phys. Chem. A 2008, 112, 12616-12621.

(13) Gerecht, E.; Douglass, K. O.; Plusquellic, D. F. Opt. Express 2011, 19, 8973-8984.

(14) Zaleski, D. P.; Neill, J. L.; Muckle, M. T.; Seifert, N. A.; Brandon Carroll, P.; Widicus Weaver, S. L.; Pate, B. H. J. Mol. Spectrosc. 2012, 280, 68-76. 
(15) Hirahara, Y.; Ohshima, Y.; Endo, Y. J. Chem. Phys. 1994, 101, 7342-7349.

(16) Ohshima, Y.; Endo, Y. J. Mol. Spectrosc. 1992, 153, 627-634.

(17) Drouin, B. J.; Maiwald, F. W.; Pearson, J. C. Rev. Sci. Instrum. 2005, 76, 093113.

(18) Kisiel, Z.; Pszczółkowski, L.; Drouin, B. J.; Brauer, C. S.; Yu, S.; Pearson, J. C.; Medvedev, I. R.; Fortman, S.; Neese, C. J. Mol. Spectrosc. 2012, 280, 134-144.

(19) Balle, T. J.; Flygare, W. H. Rev. Sci. Instrum. 1981, 52, 33-45.

(20) Harrington, H. W.; Hearn, J. R.; Rauskolb, R. F. Hewlett-Packard J. 1971, 22, 1.

(21) Remijan, A. J.; Hollis, J. M.; Jewell, P. R.; Lovas, F.; Corby, J. 2013; Vol. 221.

(22) Bernath, P. F. Spectra of Atoms and Molecules; Second.; Oxford University Press: New York, 2005.

(23) Ekkers, J.; Flygare, W. H. Rev. Sci. Instrum. 1976, 47, 448-454.

(24) Pickett, H. M.; Poynter, R. L.; Cohen, E. A.; Delitsky, M. L.; Pearson, J. C.; Müller, H. S. P. J. Quant. Spectrosc. Radiat. Transf. 1998, 60, 883-890.

(25) Richman, B.; Kachanov, A.; Paldus, B.; Strawa, A. Opt. Express 2005, 13, 33763387.

(26) Gordy, W.; Cook, R. L. Microwave Molecular Spectra; 3rd ed.; John Wiley and Sons: New York, 1984. 


\section{Chapter 5}

\section{A Chirped Pulse - Fourier Transform Millimeter wave Discharge Spectrum of Acetonitrile, $\mathrm{CH}_{3} \mathrm{CN}$}

\section{Introduction}

In the context of astronomy, molecular rotational spectroscopy has helped astronomers to identify complex organic materials in the interstellar medium (ISM) since the 1960 's. The first example of this was the detection of the hydroxyl radical in $1963^{1}$ by radio astronomy. Since that time almost 200 molecules have been detected in the $\mathrm{ISM}^{2}$ at a rate of roughly four new detections per year ${ }^{3}$. The use of laboratory spectroscopic results has been essential for these interstellar detections. Many of these detected species are highly reactive or unstable under terrestrial conditions such as $\mathrm{C}_{6} \mathrm{H}$ both as an anion ${ }^{4}$ and radical $^{5}$ and $\mathrm{CCH}^{6,7}$ and hence multiple experimental approaches for "in situ" generation and detection of such species have been developed. Since the discovery of the interstellar hydroxyl radical, techniques for the generation and characterization of interstellar molecules in the both the millimeter wave (mm-wave) and microwave regime have had much success. One of the earliest methods for generating these species was the DC glow discharge method. It was often paired with instruments in the mm-wave regime and aided in many interstellar discoveries ${ }^{5,7,8}$. At lower frequencies, one of the first methods to producing these reactive species was microwave discharge cavities ${ }^{9,10}$. Then in 1997 , McCarthy and co-workers ${ }^{11}$ presented a pulsed discharge nozzle design, based on the design by Iida and co-workers ${ }^{12}$. The design by McCarthy and co-workers was paired with their Fourier-transform microwave (FTM) spectrometer, and this combination 
has aided in the assignment of carbon chain species, ions, and others in the interstellar medium $^{3,11,13,14}$. In 2012, the discharge nozzle design was coupled with the chirped pulse-Fourier transform microwave (CP-FTMW) technique and broadband reaction screening process ${ }^{15}$, yielding several interstellar detections, such as those of trans-methyl formate $^{16}$ and ethanimine ${ }^{17}$. The CP-FTmm-wave technique discussed in Chapters 2 was developed soon after in late $2012^{18}$. By pairing the instruments in the microwave and mm-wave regimes, rotational spectroscopy has a spectral coverage over $1 \mathrm{THz}$ in which to identify molecular spectra.

Advances in laboratory based measurements in rotational spectroscopy have been paralleled by the evolution of radio astronomy. New advances in technology have allowed for better spatial and spectral resolution, and increased the data throughput. As the Atacama Large Millimeter Array (ALMA) comes online at full capacity, its data rates will increase to $1 \mathrm{~TB} /$ day. Datasets like this have created the need for faster, sensitive laboratory data collection methods. With a frequency range from $84-720 \mathrm{GHz}$ and a data rate of $\sim 400 \mathrm{~TB} / \mathrm{yr}^{19}$, ALMA will produce more spectral (and spatial) data in one year than all other radio observatories combined. This will create a backlog of data as well as the need for new analytical tools. The status quo is insufficient to be able to handle these datasets, and the speed of laboratory acquisitions and analysis is doubtless creating a bottleneck. If this problem is overcome, the rate of identification of further interstellar species could be increased, which would lead to a better understanding of the chemistry occurring in dense molecular areas such as star forming regions. In this context, and in order to try to overcome these challenges, we recently reported on the construction of a real-time acquisition segmented chirped pulsed-Fourier transform millimeter wave 
(CP-FTmm-wave) spectrometer ${ }^{20}$ operating in the $260-295 \mathrm{GHz}$ frequency range, which overlaps with frequencies in Band 6 and Band 7 of ALMA. In this chapter, the implementation of a pulsed discharge nozzle ${ }^{13}$ in the segmented CP-FTmm-wave spectrometer is presented. The capabilities of this experimental update are illustrated through the generation and analysis of products of a pulsed discharge experiment of acetonitrile, and compared to those observed in the microwave regime. The availability of this experimental approach coupled with automated data assignment from catalogs such as $\mathrm{SPLATALOGUE}^{21}, \mathrm{JPL}^{22}$, or $\mathrm{CDMS}^{23}$ will greatly assist in the interpretation and analysis of current and future radio astronomical datasets.

\section{Experimental}

The mm-wave spectrometer used for this experiment, which has been described elsewhere ${ }^{18,20}$, is briefly described here. The modifications made to the instrument to perform discharge experiments are also detailed. The segmented CP-FTmm-wave spectrometer generates two pulses, the excitation pulse and the local oscillator (LO) pulse, using a $12 \mathrm{GS} / \mathrm{s}$, two channel, Agilent M8190 arbitrary waveform generator (AWG). The entire bandwidth of the instrument is covered by the segmented approach described by Neill et al. ${ }^{24}$ and Harris ${ }^{20}$. The $720 \mathrm{MHz}$ bandwidth pulses are upconverted by a microwave circuit described by Harris ${ }^{20}$ to the specifications of the $x 24$ VDI active multiplier chain $(\mathrm{AMC})$ and the $\mathrm{x} 12$ mixAMC, mixing to final frequencies between 260-295 GHz. After the $100 \mathrm{~ns}$ excitation chirped pulse has been introduced, the free induction decay (FID) is mixed down using the LO pulse to $720-1440 \mathrm{MHz}$ and digitized on the real-time Agilent U1070A Acqiris high-speed PCI digitizer ${ }^{20}$. Each segment, in 
this segmented approach, is $2 \mu \mathrm{s}$ in duration. This spectrometer can operate in two modes; fastmode, in which the total acquisition time is reduced to $100 \mu$ s by using fifty $720 \mathrm{MHz}$ segments and high dynamic range (HDR) mode where each $720 \mathrm{MHz}$ segment is further segmented to thirty $24 \mathrm{MHz}$ segments. This increases the overall number of segments to 1500 and reduces the number of spurious signals ${ }^{20}$. The data presented were taken using HDR mode, and spectra containing a coherently time averaged set of 10,000 free induction decays (FIDs) were collected. The full $35 \mathrm{GHz}$ of bandwidth was collected in 1.5 minutes. The $40 \mathrm{~L}$ gas chamber, described by Steber et al. ${ }^{18}$ was outfitted with a pulsed discharge nozzle ${ }^{13}$ in one of the side ports for sample introduction. The voltage for the discharge electrodes was set to $1.2 \mathrm{kV}$ and the current was approximately $15 \mathrm{~mA}$. The chamber was also equipped with a cold copper trap, which was cooled to $77 \mathrm{~K}$. The looped copper tubing, approximately $200 \mathrm{~cm}$ in length and surface area of $252 \mathrm{~cm}^{2}$, was attached on one end to the vacuum chamber, while at the other end it was attached to the vacuum pump via a $1 / 4$ " Swagelok B-series valve. For the 50 mTorr experiment the chamber pressure was increased to $\sim 50$ mTorr via pulsing of the discharge nozzle, the chamber was sealed off, and the spectrum was taken. For experiments involving preconcentration, the chamber was filled to 10 Torr, closed off from vacuum and allowed to sit for 40 min to effectively freeze out $50 \%$ of the sample. The cold trap was opened to vacuum so that a steady flow of gas from the chamber would enter the trap and freeze out. After the 40 minutes, the trap was closed off from the chamber and the vacuum and allowed to warm up. The chamber was evacuated of residual gas and filled with the gas from the cold trap. The pressure was monitored by three MKS Baratron pressure gauges: 
50 mTorr head (627B-22683), 1 Torr head (627001TDC2B), and the 100 Torr head (627B12TDC28) so that various pressures could be monitored.

The microwave data set was acquired using the 6-18 GHz CP-FTMW spectrometer equipped with three pulsed discharge nozzles as described elsewhere ${ }^{25,26}$. The pulse was extended up to $19 \mathrm{GHz}$ to allow the detection of the $\mathrm{J}=1-0$ transition of $\mathrm{CH}_{3} \mathrm{CN}$, and 330,000 FID were acquired. The electrical discharge was set to a voltage of $1.2 \mathrm{kV}$. The sample for pressure dependence and trapping efficiency was a mixture of $1 \%$ propyne $\left(\mathrm{CH}_{3} \mathrm{CCH}\right)$ in Ne. The mm-wave discharge sample was a tank of $0.1 \%$ acetonitrile $\left(\mathrm{CH}_{3} \mathrm{CN}\right.$, a highly abundant interstellar molecule) in Ne mixture. For the microwave study, a tank of $0.4 \% \mathrm{CH}_{3} \mathrm{CN}$ diluted in Ne was made. The pressure behind the nozzle was 20 pounds per square inch (PSI) in both experiments. The $98 \% \mathrm{CH}_{3} \mathrm{CCH}$ was obtained from Sigma Aldrich and the $\geq 98.5 \% \mathrm{CH}_{3} \mathrm{CN}$ was purchased from Fischer Scientific. Both were used without further purification.

\section{Results}

One of the goals of the discharge experiment was to characterize the weak species that were generated. To do so, the instrument needed to be operating in the Doppler broadening regime. As most of the experiments performed on this spectrometer to date have been low pressure experiments, it was necessary to determine the pressure at which Doppler broadening ceased to be the dominant effect. The benefits of working in the Doppler broadening regime are numerous and are vital to identifying weak species. The more accurate line centers associated with the Doppler regime allow for a more certain 
identification of weak interstellar species, and the narrow line shapes allow closely spaced molecular transitions to be distinguishable from one another. This becomes important when line density is high and molecular intensities differ by orders of magnitude, which is the case in the present experiment. The other benefit is that the collisional decay rate $\left(\mathrm{T}_{2}\right)$ is slower, and the FID decay lasts longer. In order to monitor the effect of signal intensity versus pressure, the signal level of the $17_{3}-16_{3}$ transition of propyne $(290452.217 \mathrm{MHz})$ was monitored as the pressure of a $1 \%$ sample of propyne in Ne was varied from 1 mTorr up to 500 mTorr. Propyne was chosen due to the fact that it is a relatively benign molecule and does not adsorb onto the walls of the static cell. The results are shown in Fig. 5.1. A linear regression was performed on the data points from 1 to $30 \mathrm{mTorr}$ and is plotted in red in the inset of the graph. As is expected, the signal intensity increases linearly. However, this is only the case up to a pressure of 25 mTorr. After 25 mTorr, pressure broadening starts to suppress the signal intensity.

The other characteristic of the instrument that needed to be measured was the effectiveness of the cold trapping and was first tested with propyne. The signal level of $1 \mathrm{mTorr}$ of pure propyne was measured, after which $100 \mathrm{mTorr}$ of $1 \%$ propyne in Ne was placed into the chamber. The chamber was closed off, liquid nitrogen was used to cool the trap, and the trap was then opened to the vacuum pump. After the allotted period of time, the trap was closed, isolated from the chamber, warmed and the resulting gas was introduced back into the chamber. The signal level of propyne was measured after different durations, and it was found that the effective trapping was $50 \%$ after $40 \mathrm{~min}$, $64 \%$ after $60 \mathrm{~min}$, and $89 \%$ after $90 \mathrm{~min}$. 


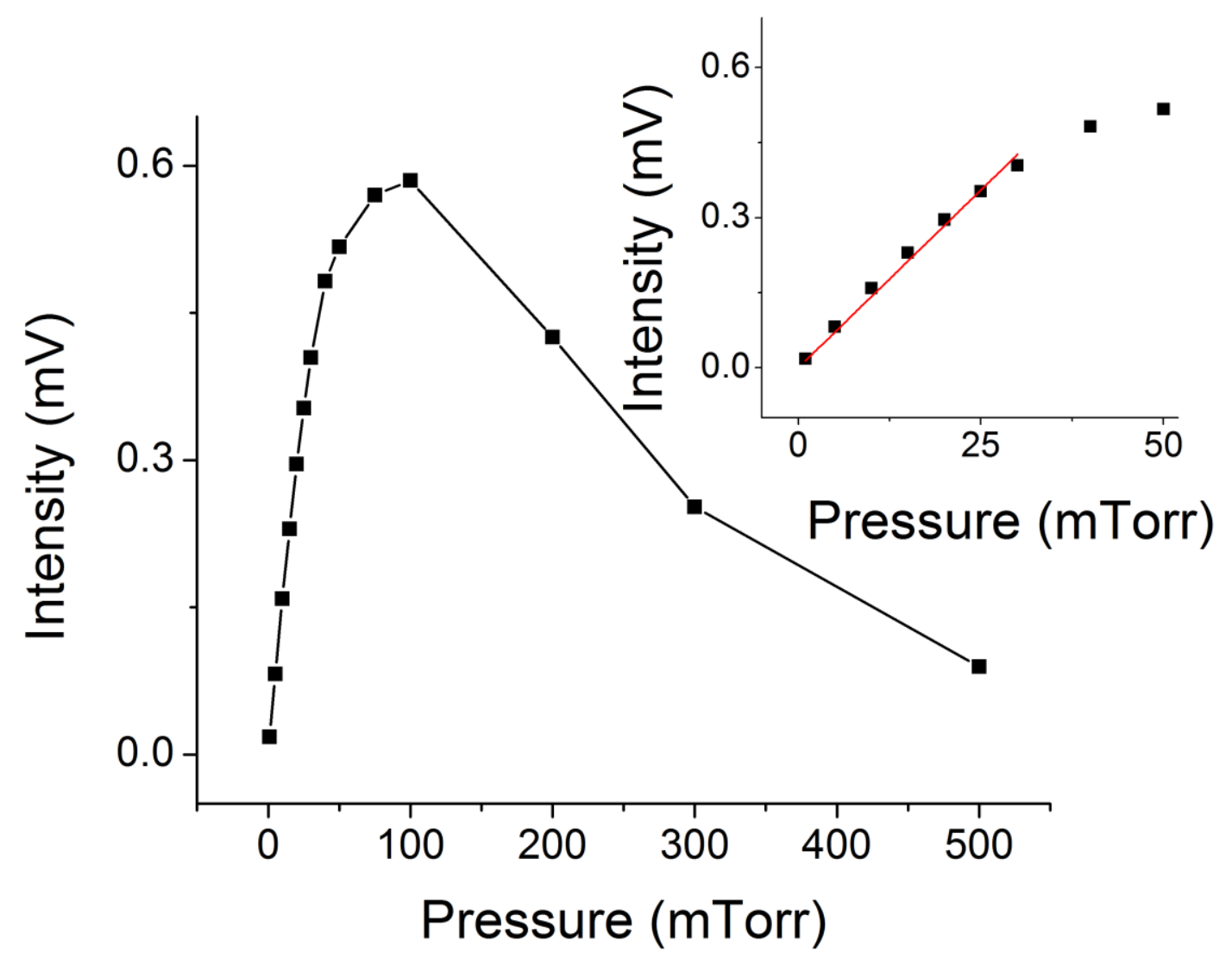

Figure 5.1. The intensity of the propyne $17_{3}-16_{3}$ transition at $290452.217 \mathrm{MHz}$ as a function of pressure, from $1 \mathrm{mTorr}$ to 500 mTorr. Each measurement was a total of 10,000 averages. The inset displays the intensity versus the pressure from $1 \mathrm{mTorr}$ to 50 mTorr. As can be seen from the inset, the signal level goes up linearly with pressure until roughly 25 mTorr, at which point pressure broadening becomes the main contributor to the line shape. The fit for through 30 mTorr is $y=0+0.01421 x$ with an $R^{2}$ of 0.99669 . 
In order to determine if the pulsed discharge nozzle would work efficiently in the mm-wave setup, a discharge spectrum was taken of the $0.1 \% \mathrm{CH}_{3} \mathrm{CN}$ in $\mathrm{Ne}$ at a pressure of $50 \mathrm{mT}$ Torr. This gave an effective concentration of $\mathrm{CH}_{3} \mathrm{CN}$ in the chamber of $50 \mu$ Torr. The top panel of Fig. 5.2 shows the $35 \mathrm{GHz}$ wide HDR spectrum of the discharge spectrum both with the discharge on (black trace) and off (red trace). To further illustrate the success of the discharge, the bottom panel shows an inset of the $\mathrm{J}=3-2$ transition hydrogen cyanide $(\mathrm{HCN})$ at $265886.424 \mathrm{MHz}(\mathrm{left}) . \mathrm{HCN}$ was seen at a SNR of 900:1, and cyanoacetylene $\left(\mathrm{HC}_{3} \mathrm{~N}\right)$, the next most abundant product, had an SNR of 12.5:1. The right inset of the bottom panel shows the absence of vinyl cyanide, which is an expected product of the $\mathrm{CH}_{3} \mathrm{CN}$ discharge. The production of vinyl cyanide was too low to observe, thus necessitating the use of cold trapping.

Because the concentration of the less abundant discharge species was too low, cold trapping was employed as described earlier. Using this method, the SNR of HCN increased to $17,500: 1$ and vinyl cyanide is visible at an SNR of 81:1, which can be seen in Fig. 5.3. Table 5.1 lists the potential discharge products that could be made from discharging $\mathrm{CH}_{3} \mathrm{CN}$, and whether the species was detected in the microwave and/or the mm-wave spectrum. From Table 5.1 it can be seen that only four species were present in both spectra; $\mathrm{CH}_{3} \mathrm{CN}, \mathrm{HC}_{3} \mathrm{~N}, \mathrm{CH}_{2} \mathrm{CHCN}$, and $\mathrm{CH}_{3} \mathrm{CH}_{2} \mathrm{CN}$. In order to compare the efficiency of the discharge nozzle in the mm-wave setup, the relative abundances of these molecules in comparison to the reactant, $\mathrm{CH}_{3} \mathrm{CN}$, were calculated for both the mm-wave spectrum and for the microwave spectrum. The sample consumption for these spectra 

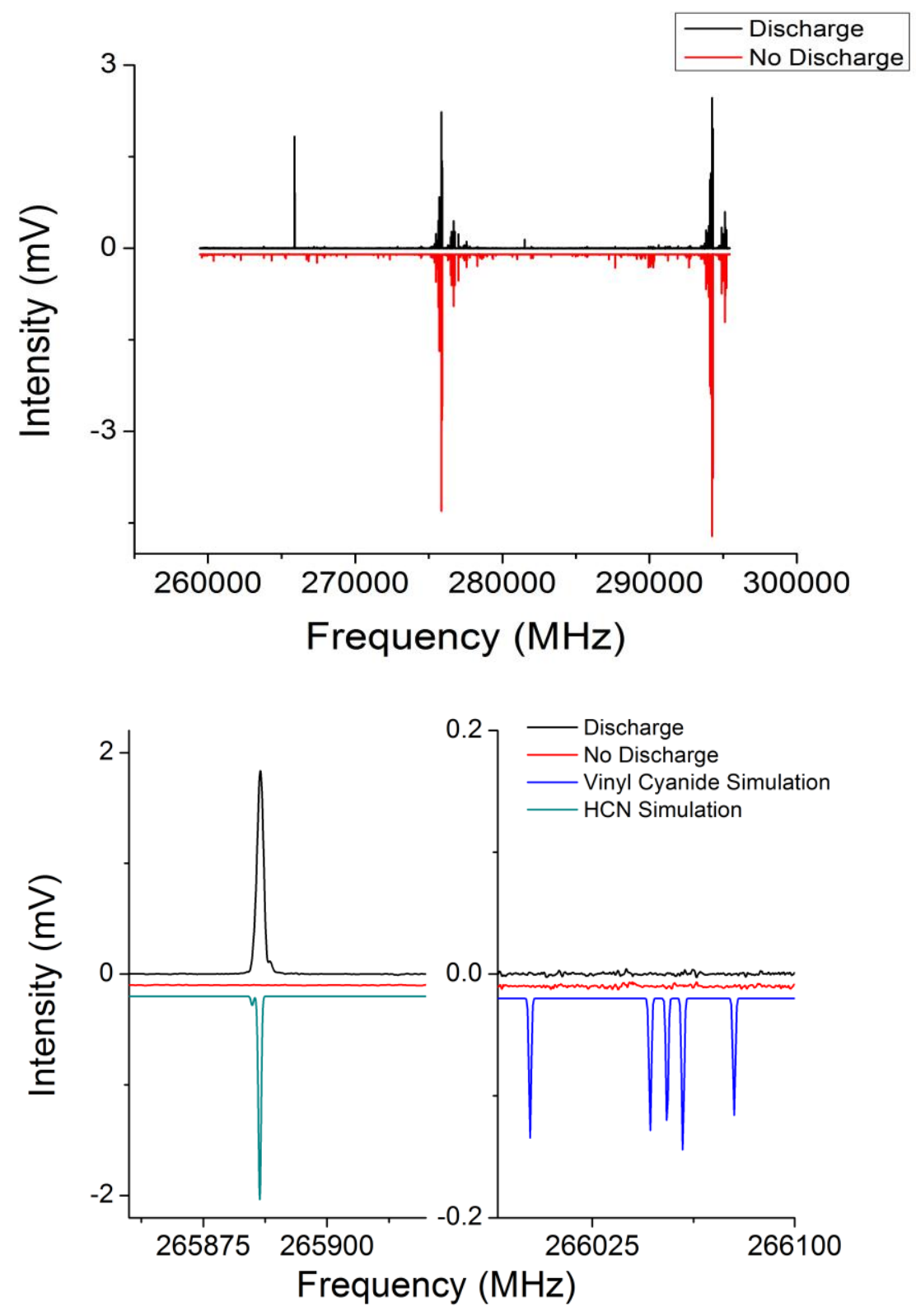

Figure 5.2. The top panel shows a 10,000 average full segmented chirped-pulse millimeter wave spectrum from $260-295 \mathrm{GHz}$ of $\mathrm{CH}_{3} \mathrm{CN}$ in Ne. The top spectrum shows the $\mathrm{CH}_{3} \mathrm{CN}$ in Ne spectrum with a ca. $1.2 \mathrm{kV}$ discharge being applied to the sample as it is pulsed into the chamber, while the red trace shows the spectrum without the discharge being applied. The pressure inside the chamber was 50 mTorr when the spectrum was acquired. The bottom panel shows an inset of both the $J=3-2$ transition of HCN (left) and several transitions of the $J=28-27$ of vinyl cyanide (right) that are expected to be seen in the discharge spectrum. Simulations were made from $\mathrm{JPL}^{22}$. 


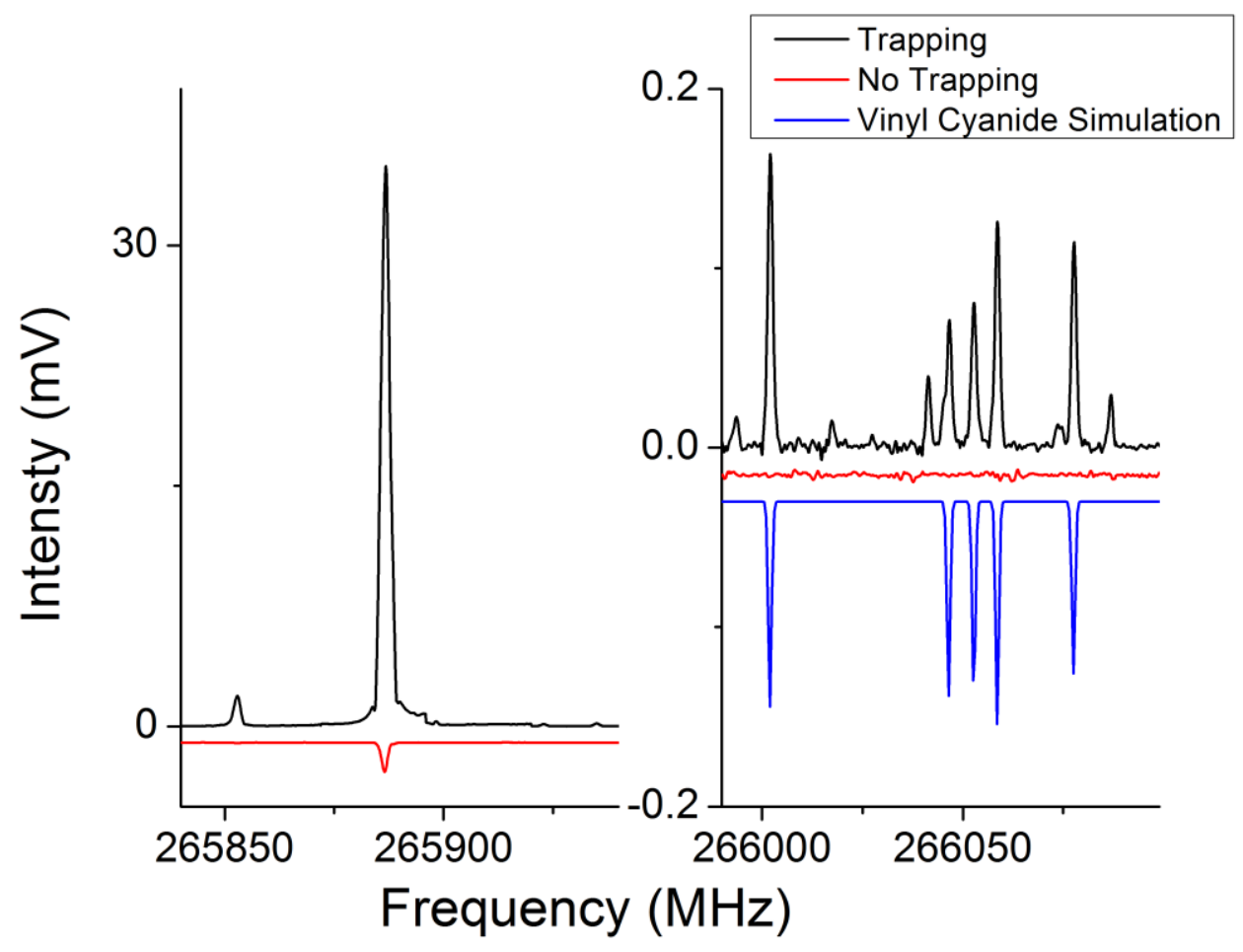

Figure 5.3. The left panel shows the $\mathrm{J}=3-2$ transition of $\mathrm{HCN}$ after trapping (black) had been implemented versus the signal intensity of the non-trapped spectrum (red). Both spectra were acquired for 10,000 averages. The right panel again shows the transitions of vinyl cyanide upon trapping (black) and non-trapping (red) in comparison to the simulation $^{22}$ (blue). As can be seen, an appreciable amount of vinyl cyanide is not seen until trapping has been implemented at which point the $\mathrm{S} / \mathrm{N}$ ratio for the $28_{10,19}-27_{10,18}$ transition at $266002.002 \mathrm{MHz}$ is $81: 1$. 
Table 5.1. Displayed is a list of potential molecules that could be observed in a discharge spectrum of $\mathrm{CH}_{3} \mathrm{CN}$. The molecules presence in either the mm-wave spectrum, microwave spectrum, or both is indicated and also whether the molecule has been detected in the interstellar medium. Molecules seen in both the microwave region and the millimeter wave region are highlighted in bold.

\begin{tabular}{|c|c|c|c|}
\hline Molecule & $260-295 \mathrm{GHz}$ & $6-18 \mathrm{GHz}$ & Interstellar? \\
\hline $\mathrm{HCN}$ & Yes & No & Yes \\
\hline $\mathrm{HCN} \mathrm{V}_{2}=1$ & Yes & No & Yes $^{27,28}$ \\
\hline $\mathrm{HCN} \mathrm{V}_{2}=2$ & Yes & No & $\mathrm{Yes}^{27,28}$ \\
\hline $\mathrm{HNC}$ & No & No & Yes \\
\hline $\mathrm{CH}_{3} \mathrm{CN}$ & Yes & Yes & Yes \\
\hline $\mathrm{HNC}_{3}$ & No & No & Yes $^{29,30}$ \\
\hline $\mathrm{HCCNC}$ & No & Yes & Yes $^{30,31}$ \\
\hline $\mathrm{HC}_{3} \mathrm{~N}$ & Yes & Yes & Yes \\
\hline $\mathrm{HC}_{3} \mathrm{~N} \mathrm{v}_{7}=1$ & Yes & No & $\mathrm{Yes}^{32-35}$ \\
\hline $\mathrm{HC}_{3} \mathrm{~N} \mathrm{v}_{7}=2$ & Yes & No & $\mathrm{Yes}^{32,34}$ \\
\hline $\mathrm{HC}_{3} \mathrm{~N} \mathrm{v}_{6}=1$ & Yes & No & $\mathrm{Yes}^{32,34,35}$ \\
\hline $\mathrm{HC}_{3} \mathrm{~N} \mathrm{v}_{6}=2$ & Yes & No & $\mathrm{Yes}^{32,34}$ \\
\hline $\mathrm{HC}_{3} \mathrm{~N} \mathrm{v}_{6}=1, \mathrm{v}_{7}=1$ & Yes & No & Yes $^{32,34}$ \\
\hline $\mathrm{HC}_{3} \mathrm{~N} \mathrm{v}_{4}=1$ & Yes & No & Yes $^{32,34}$ \\
\hline $\mathrm{HC}_{3} \mathrm{~N} \mathrm{v}_{4}=1, \mathrm{v}_{7}=1$ & Yes & No & Yes $^{32,34}$ \\
\hline $\mathrm{HC}_{3} \mathrm{~N} \mathrm{v}_{5}=1 / \mathrm{v}_{7}=3$ & Yes & No & $\mathrm{Yes}^{34}$ \\
\hline $\mathrm{H}^{13} \mathrm{CCCN}$ & Yes & No & Yes \\
\hline $\mathrm{HC}^{13} \mathrm{CCN}$ & Yes & No & Yes \\
\hline $\mathrm{HCC}^{13} \mathrm{CN}$ & Yes & No & Yes \\
\hline $\mathrm{HCCC}^{15} \mathrm{~N}$ & Yes & No & Yes \\
\hline $\mathrm{H}^{13} \mathrm{CCCN} \mathrm{v}_{7}=1$ & Yes & No & $\mathrm{Yes}^{32}$ \\
\hline $\mathrm{HC}^{13} \mathrm{CCN} \mathrm{v}_{7}=1$ & Yes & No & Yes $^{32,34}$ \\
\hline $\mathrm{HCC}^{13} \mathrm{CN} \mathrm{v}_{7}=1$ & Yes & No & $\mathrm{Yes}^{32,34}$ \\
\hline $\mathrm{CH}_{2} \mathrm{CHCN}$ & Yes & Yes & Yes \\
\hline $\mathrm{E} \& \mathrm{Z}-\mathrm{CH}_{3} \mathrm{CHNH}$ & No & Yes & Yes \\
\hline $\mathrm{CH}_{3} \mathrm{CH}_{2} \mathrm{CN}$ & Yes & Yes & Yes \\
\hline
\end{tabular}


was $22 \mu \mathrm{mol}$ of $\mathrm{CH}_{3} \mathrm{CN}$ and $4.8 \mathrm{mmol}$ respectively, and the time for spectral acquisition was 1.5 min (plus pre-concentration) versus three hours. The results for this comparison are shown in Table 5.2. The relative abundances of $\mathrm{HC}_{3} \mathrm{~N}$ and $\mathrm{CH}_{2} \mathrm{CHCN}$ are comparable in the two experiments, while the abundance of $\mathrm{CH}_{3} \mathrm{CH}_{2} \mathrm{CN}$ is larger by an order of magnitude in the mm-wave spectrum. Besides this difference, we are able to observe vibrationally excited states of both $\mathrm{HCN}$ and $\mathrm{HC}_{3} \mathrm{~N}$ in the mm-wave spectrum. The bending modes $\mathrm{v}_{2}=1$ and $\mathrm{v}_{2}=2$ of $\mathrm{HCN}$ were observed. For $\mathrm{HC}_{3} \mathrm{~N}$, several bending modes (two fundamentals and two overtones), a stretching mode, and two combination bands of $\mathrm{HC}_{3} \mathrm{~N}$ were observed. A Fermi resonance system, the $\mathrm{v}_{5}=1 / \mathrm{v}_{7}=3$, was also observed. The observed transition intensities go in the order of $(0,0,0,1)>(0,0,0,2)>$ $(0,0,1,0)>(0,1,0,0) /(0,0,0,3)>(0,0,1,1)>(1,0,0,0)>(0,0,2,0)>(1,0,0,1)$, in which the four numbers refer to the vibrational state of the four modes $\left(\mathrm{v}_{4}, \mathrm{~V}_{5}, \mathrm{~V}_{6}, \mathrm{~V}_{7}\right)$. This is in good agreement with what would be expected from the experimental vibrational frequencies previously reported ${ }^{36}$. The frequencies for the vibrationally excited states of $\mathrm{HC}_{3} \mathrm{~N}$ were identified due to the extensive amount of work that has been done on $\mathrm{HC}_{3} \mathrm{~N}$ from the microwave up to $1 \mathrm{THz}^{36-43}$. The experimental vibrationally excited states of $\mathrm{HC}_{3} \mathrm{~N}$ are shown in Fig. 5.4 for the $\mathrm{J}=30-29$ transition. A list of all newly measured transitions can be found in Appendix B. It is interesting to note that the isomer $\mathrm{HCCNC}$ of $\mathrm{HC}_{3} \mathrm{~N}$ was observed in the microwave spectrum; however, it was not observed in the mm-wave spectrum. 
Table 5.2. For the four molecules that were observed in both spectra, the reactant and the three products, a comparison of the molecule's relative abundance to the starting material is shown. The abundance of $\mathrm{CH}_{3} \mathrm{CN}$ was scaled normalized to 100 and the relative abundance to $\mathrm{CH}_{3} \mathrm{CN}$ for the other three molecules is shown. It can be seen that for $\mathrm{HCCCN}$ and $\mathrm{CH}_{2} \mathrm{CHCN}$ the relative abundances in the microwave and millimeter wave are around the same order of magnitude, while $\mathrm{CH}_{3} \mathrm{CH}_{2} \mathrm{CN}$ has a higher abundance in the millimeter wave spectrum.

\begin{tabular}{lcc}
\hline Molecule & $\begin{array}{c}260-295 \mathrm{GHz} \text { Relative } \\
\text { Abundances }\end{array}$ & $\begin{array}{c}\text { 6-19 GHz Relative } \\
\text { Abundances }\end{array}$ \\
\hline $\mathrm{CH}_{3} \mathrm{CN}$ & 100.0 & 100.0 \\
$\mathrm{HC}_{3} \mathrm{~N}$ & 3.0 & 2.1 \\
$\mathrm{CH}_{2} \mathrm{CHCN}$ & 2.3 & 1.6 \\
$\mathrm{CH}_{3} \mathrm{CH}_{2} \mathrm{CN}$ & 1.6 & 0.2 \\
\hline
\end{tabular}



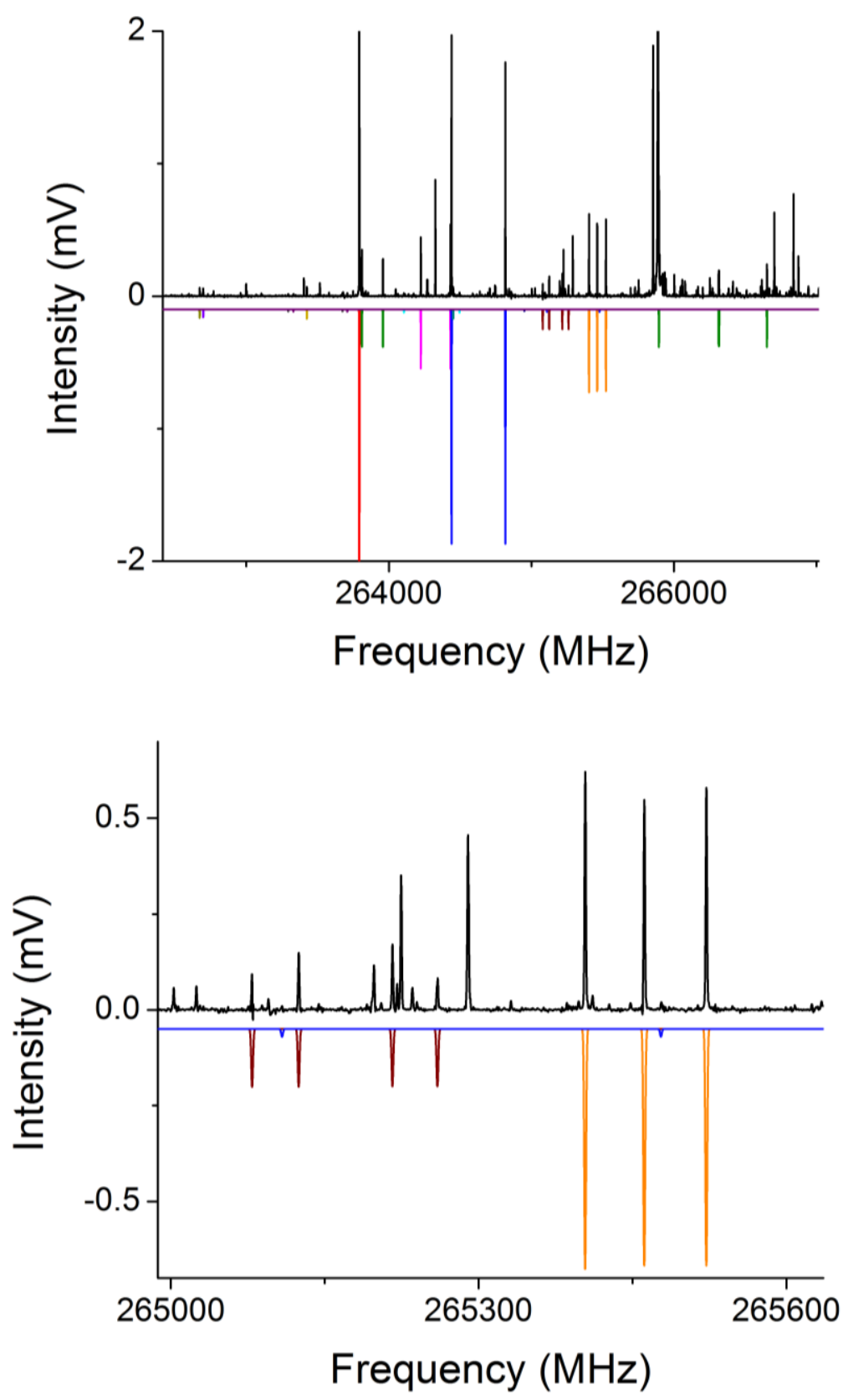

Figure 5.4. The top panel shows the trapped, discharge spectrum in comparison to the simulations for the $\mathrm{J}=29-28$ transition of cyanoacetylene, along with the vibrationally excited states, isotopologues, and the vibrationally excited states $v_{7}=1$ of the ${ }^{13} \mathrm{Cs}$. The bottom panel displays the comparison of the trapped discharge spectrum (black) to the simulations of the vibrationally excited states $\mathrm{v}_{7}=2$ (orange), $\mathrm{v}_{6}=1, \mathrm{v}_{7}=1$ (maroon), and the $\mathrm{H}^{13} \mathrm{CCCN}_{7}=1$ (navy) for $\mathrm{J}=29-28$. 


\section{Discussion}

Since the instrument is not operating in the Doppler broadening regime after 25 mTorr, it is important to keep the pressures in the chamber below that. When looking at the products that were seen in the $6-19 \mathrm{GHz}$ spectrum, it was expected that in 50 mTorr of the gas mixture, cyanoacetylene $\left(\mathrm{HC}_{3} \mathrm{~N}\right)$ would be at a signal-to-noise ratio (SNR) of more than 10:1. It was also expected that hydrogen cyanide $(\mathrm{HCN})$ would be observable as there would be a high abundance of $\mathrm{H}$ and ${ }^{\circ} \mathrm{CN}$ in the discharge plume; however, the abundance of the reactants needed to observe vinyl cyanide $\left(\mathrm{CH}_{2} \mathrm{CHCN}\right)$ and ethyl cyanide $\left(\mathrm{CH}_{3} \mathrm{CH}_{2} \mathrm{CN}\right)$ would not be sufficient. The bottom panel of Fig. 5.2 shows that this was the case. In order to work at pressures in the linear regime and be able to see the weaker species, preconcentration was implemented. The efficiency of this design is demonstrated in Table 5.2. The abundances for two of the three products are in good agreement between the two datasets, and $\mathrm{CH}_{3} \mathrm{CH}_{2} \mathrm{CN}$ is an order of magnitude more abundant in the mm-wave dataset. This demonstrates the ability of the mm-wave technique to offer the same sensitivity in measuring weak discharge species as that of the microwave method. Ethanimine ${ }^{17}$ and cyanomethanimine ${ }^{26}$ were detected by mixing $\mathrm{H}_{2} \mathrm{~S}$ with $\mathrm{CH}_{3} \mathrm{CN}$ and $\mathrm{NH}_{3}$ with $\mathrm{CH}_{3} \mathrm{CN}$ respectively, and running these mixtures through the discharge nozzles. They were measured from 8-40 GHz. Relatively weak species like these should therefore be detectable in the mm-wave instrument, based on our present relative abundance results.

The results of the mm-wave spectra (especially the high abundance of $\mathrm{CH}_{3} \mathrm{CH}_{2} \mathrm{CN}$ in comparison to what was observed in the microwave) indicate that these two systems are different. The bulk of the reactions that are occurring in the microwave 
experiment are most likely occurring after the electrodes in the nozzle and as the expansion starts. The nozzle is then never in contact with the sample again. In the mmwave experiment, the same reactions are occurring in the nozzle; however, once the gas has expanded into the chamber, the chamber begins to fill up to higher pressures than those seen in the microwave pulsed jet experiments. The current from the discharge nozzle has the opportunity to arc to the gas surrounding the nozzle, where it would interact with remaining reactants. This could cause a higher concentration of ethyl cyanide as it is thought that the reaction of formation is between ${ }^{\circ} \mathrm{CH}_{2} \mathrm{CN}$ and ${ }^{\circ} \mathrm{CH}_{3}$. The remaining gas in the chamber would then stabilize the ethyl cyanide so that it did not undergo dehydrogenation to form vinyl cyanide and cyanoacetylene.

Another different between these two spectrometers is that the mm-wave experiment is under thermodynamic control, unlike the kinetic control that we experience in microwave experiments. While the molecules are formed in a supersonic expansion in the microwave experiment and never allowed to come to equilibrium, the sample in the mm-wave experiment is at room temperature and is given ample time to equilibrate. This is important in the formation of isomers. As stated before, the HCCNC isomer is seen in the microwave experiments, whereas only the $\mathrm{HC}_{3} \mathrm{~N}$ isomer is found in the mm-wave experiment. The energy difference between the two isomers is approximately $28.24 \mathrm{kcal} \mathrm{mol}^{-1} 44$, and the inter-conversion of the two isomers goes through a transition state that is $65.26 \mathrm{kcal} \mathrm{mol}^{-1}$ higher than cyanoacetylene ${ }^{44}$. It is plausible that HCCNC interconverts back to the more stable $\mathrm{HC}_{3} \mathrm{~N}$ in the thermodynamic conditions of the mmwave experiment. In order to study more exotic and reactive species such as ions and radicals, the instrument setup described here could be modified. With the availability of 
real-time data collection, and the fact that the lifetime of radicals in the mm-wave regime has been shown to be over $1 \mathrm{~ms}^{45}$, monitoring the concentration and reactions of radicals as a function of time should be possible.

Several of the molecules presented in this work have been previously studied by mm-wave methods. These techniques have relied on single frequency light sources such as klystrons, Gunn oscillators, and spectrally pure synthesizers. The implementation of the present technique offers a frequency agile source able to sweep large bandwidths $(35 \mathrm{GHz})$ in times as short as $100 \mu \mathrm{s}$. This allows us to acquire a spectrum over a spectral range of $260-295 \mathrm{GHz}$ with high sensitivity in $1.5 \mathrm{~min}$. The discharge spectrum of $\mathrm{CH}_{3} \mathrm{CN}$ presented has a dynamic range of the strongest species $\left(\mathrm{CH}_{3} \mathrm{CN}\right)$ of $27,000: 1$ and the discharge generated 22 species that were absent when the discharge was not applied. New species could be detected by creating a reactant mixture of prevalent interstellar molecules. Spectral acquisition could take little more than a press of a button, with modifications to the current setup that are being pursued.

It has been shown by Fortman et al. ${ }^{46}$ that in mm-wave and sub-mm-wave room temperature spectra, the complexity of the spectra is often due to other constituents such as vibrationally excited states, or so called "weeds". This is evident in the current spectrum by the presence of 4 isotopologues and 11 vibrationally excited states of $\mathrm{HC}_{3} \mathrm{~N}$, 2 vibrationally excited states of $\mathrm{HCN}$, and 180 unidentified lines. Nothing has been assigned for the unidentified lines (U-lines) as of yet, but the strongest U-line is 175 times weaker than HCN. Automated spectral assigning tools, such as autofit ${ }^{47}$, may aid in the assignment of these U-lines. Beyond unidentified molecular assignments, a large push 
has been made to automate the instrument for not only data acquisition, but also the data analysis of known species. By pairing the CP-FTmm-wave method with such automated analysis tools, both the data acquisition and analysis time is reduced significantly. The peaks in the discharge spectrum are identified by querying databases such as SPLATALOGUE ${ }^{21}$. The analysis time can be further reduced by doing a subtraction of the spectra obtained with the electric discharge on and one with the discharge off so that unidentified "weeds" from the reactants are not a hindrance. While contamination from adsorbed material on the cell walls is a concern, adequate pumping can remove most of the contaminants below a level that would be detectable for this instrument; this problem can be overcome in the cold trap with heating, but during the present experiments, it did not seem to be a problem. If the adsorbed molecules somehow did make their way into the gas sample, the subtraction procedure mentioned above would remove them from the spectrum. Implementation of these techniques will reduce the time required to analyze the ALMA datatsets and others like them.

\section{Conclusions}

A segmented CP-FTmm-wave spectrometer operating between 260-295 GHz was successfully modified to do static cell, pulsed discharge experiments allowing extension of studies carried out at lower frequencies the work that has been done at lower frequencies. Over 20 species were identified in the discharge spectrum of $\mathrm{CH}_{3} \mathrm{CN}$, including $\mathrm{HCN}, \mathrm{HC}_{3} \mathrm{~N}$ (and their vibrationally excited states) showing that this is a viable method, and it is no longer necessary to have multiple discharge setups in order to study discharge reactions at different wavelengths. The ease and relatively fast spectral 
acquisition times of this spectrometer make it ideal not only for interstellar detections, but with slight modifications in both the data processing and sample creation, it can be expanded to study reaction kinetics and characterize reactive species such as radicals. The high sensitivity of the spectrometer will allow for the removal of "weeds" in astronomical datasets, making their analysis easier. By coupling this with automated spectral line identification, the bottleneck preventing timely analysis of the large datasets becoming available from ALMA will ease. 


\section{Chapter 5 References}

(1) Weinreb, S.; Barrett, A. H.; Meeks, M. L.; Henry, J. C. Nature 1963, 200, 829-831.

(2) Woon, D. E. The Astrochymist http://astrochymist.org/astrochymist_ism.html (accessed Oct 1, 2014).

(3) Thaddeus, P.; McCarthy, M. C. Spectrochim. Acta. A. Mol. Biomol. Spectrosc. 2001, 57, 757-774.

(4) McCarthy, M. C.; Gottlieb, C. A.; Gupta, H.; Thaddeus, P. Astrophys. J. Lett. 2006, 652, L141.

(5) Pearson, J. C.; Gottlieb, C. A.; Woodward, D. R.; Thaddeus, P. Astron. Astrophys. 1988, 189, L13-L15.

(6) Woodward, D. R.; Pearson, J. C.; Gottlieb, C. A.; Guelin, M.; Thaddeus, P. Astron. Astrophys. 1987, 186, L14-L16.

(7) Gottlieb, C. A.; Gottlieb, E. W.; Thaddeus, P. Astrophys. J. 1983, 264, 740-745.

(8) Gottlieb, C. A.; Gottlieb, E. W.; Thaddeus, P.; Kawamura, H. Astrophys. J. 1983, $275,916-921$.

(9) Fehsenfeld, F. C.; Evenson, K. M.; Broida, H. P. Rev. Sci. Instrum. 1965, 36, $294-$ 298.

(10) Ehrenstein, G.; Townes, C. H.; Stevenson, M. J. Phys. Rev. Lett. 1959, 3, 40-41.

(11) McCarthy, M. C.; Travers, M. J.; Kovács, A.; Gottlieb, C. A.; Thaddeus, P. Astrophys. J. Suppl. Ser. 1997, 113, 105.

(12) Iida, M.; Ohshima, Y.; Endo, Y. Astrophys. J. Lett. 1991, 371, L45.

(13) McCarthy, M. C.; Chen, W.; Travers, M. J.; Thaddeus, P. Astrophys. J. Suppl. Ser. 2000, 129,611 . 
(14) McCarthy, M. C.; Thaddeus, P. Chem. Soc. Rev. 2001, 30, 177-185.

(15) Zaleski, D. P.; Stephens, S. L.; Walker, N. R. Phys. Chem. Chem. Phys. 2014.

(16) Neill, J. L.; Muckle, M. T.; Zaleski, D. P.; Steber, A. L.; Pate, B. H.; Lattanzi, V.; Spezzano, S.; McCarthy, M. C.; Remijan, A. J. Astrophys. J. 2012, 755, 153.

(17) Loomis, R. A.; Zaleski, D. P.; Steber, A. L.; Neill, J. L.; Muckle, M. T.; Harris, B. J.; Hollis, J. M.; Jewell, P. R.; Lattanzi, V.; Lovas, F. J.; Oscar Martinez, J.; McCarthy, M. C.; Remijan, A. J.; Pate, B. H.; Corby, J. F. Astrophys. J. Lett. 2013, 765, L9.

(18) Steber, A. L.; Harris, B. J.; Neill, J. L.; Pate, B. H. J. Mol. Spectrosc. 2012, 280, 310.

(19) Lacy, M.; Halstead, D. ALMA data rates and archiving at the NAASC, 2012.

(20) Harris, B. J. A Chirped Pulse Fourier Transform Millimeter Wave Spectrometer for Room Temperature, Gas Mixture Analysis. Doctor of Philosophy, University of Virginia, 2014.

(21) Remijan, A. J. Splatalogue database for astronomical spectroscopy http://www.cv.nrao.edu/php/splat/ (accessed Oct 26, 2014).

(22) Pickett, H. M.; Poynter, R. L.; Cohen, E. A.; Delitsky, M. L.; Pearson, J. C.; Müller, H. S. P. J. Quant. Spectrosc. Radiat. Transf. 1998, 60, 883-890.

(23) Müller, H. S. P.; Schlöder, F.; Stutzki, J.; Winnewisser, G. J. Mol. Struct. 2005, 742, $215-227$.

(24) Neill, J. L.; Harris, B. J.; Steber, A. L.; Douglass, K. O.; Plusquellic, D. F.; Pate, B. H. Opt. Express 2013, 21, 19743-19749. 
(25) Brown, G. G.; Dian, B. C.; Douglass, K. O.; Geyer, S. M.; Shipman, S. T.; Pate, B. H. Rev. Sci. Instrum. 2008, 79, 053103.

(26) Zaleski, D. P.; Seifert, N. A.; Steber, A. L.; Muckle, M. T.; Loomis, R. A.; Corby, J. F.; Oscar Martinez, J.; Crabtree, K. N.; Jewell, P. R.; Hollis, J. M.; Lovas, F. J.; Vasquez, D.; Nyiramahirwe, J.; Sciortino, N.; Johnson, K.; McCarthy, M. C.; Remijan, A. J.; Pate, B. H. Astrophys. J. Lett. 2013, 765, L10.

(27) Ziurys, L. M.; Turner, B. E. Astrophys. J. Lett. 1986, 300, L19-L23.

(28) Cernicharo, J.; Agúndez, M.; Kahane, C.; Guélin, M.; Goicoechea, J. R.; Marcelino, N.; De Beck, E.; Decin, L. Astron. Astrophys. 2011, 529, L3.

(29) Kawaguchi, K.; Takano, S.; Ohishi, M.; Ishikawa, S.-I.; Miyazawa, K.; Kaifu, N.; Yamashita, K.; Yamamoto, S.; Saito, S.; Ohshima, Y.; Endo, Y. Astrophys. J. Lett. 1992, 396, L49-L51.

(30) Gensheimer, P. D. Astrophys. Space Sci. 1997, 251, 199-202.

(31) Kawaguchi, K.; Ohishi, M.; Ishikawa, S.-I.; Kaifu, N. ApJ 1992, 396, L51-L53.

(32) Wyrowski, F.; Schilke, P.; Walmsley, C. M. Astron. Astrophys. 1999, 341, 882-895.

(33) Clark, F. O.; Brown, R. D.; Godfrey, P. D.; Storey, J. W. V.; Johnson, D. R. Astrophys. J. Lett. 1976, 210, L139-L140.

(34) Wyrowski, F.; Schilke, P.; Thorwirth, S.; Menten, K. M.; Winnewisser, G. Astrophys. J. 2003, 586, 344.

(35) Costagliola, F.; Aalto, S. Astron. Astrophys. 2010, 515, A71.

(36) Mallinson, P. D.; Fayt, A. Mol. Phys. 1976, 32, 473-485.

(37) Thorwirth, S.; Müller, H. S. P.; Winnewisser, G. J. Mol. Spectrosc. 2000, 204, 133144. 
(38) Thorwirth, S.; Müller, H. S. P.; Winnewisser, G. Phys. Chem. Chem. Phys. 2001, 3, $1236-1241$.

(39) Creswell, R. A.; Winnewisser, G.; Gerry, M. C. L. J. Mol. Spectrosc. 1977, 65, 420429.

(40) Yamada, K. M. T.; Creswell, R. A. J. Mol. Spectrosc. 1986, 116, 384-405.

(41) Mallinson, P. D.; de Zafra, R. L. Mol. Phys. 1978, 36, 827-843.

(42) De Zafra, R. L. Astrophys. J. 1971, 170, 165.

(43) DeLeon, R. L.; Muenter, J. S. J. Chem. Phys. 1985, 82, 1702-1704.

(44) Yang, X.; Maeda, S.; Ohno, K. Chem. Phys. Lett. 2006, 418, 208-216.

(45) Kolbe, W. F.; Leskovar, B. Rev. Sci. Instrum. 1985, 56, 1577-1581.

(46) Fortman, S. M.; Medvedev, I. R.; Neese, C. F.; Lucia, F. C. D. Astrophys. J. 2010, $725,1682$.

(47) Seifert, N. A. Broadband Microwave Spectroscopy and Automated Analysis of 12 Conformers of 1-Hexanal, 2014. 


\section{Appendix B.}

Shown below are the tabulated measured frequencies for cyanoacetylene, its isotopologues, and the vibrationally excited states.

\begin{tabular}{cccc}
$\mathrm{J}^{\prime}$ & $\mathrm{J} "$ & $\ell$ & Observed Frequency $(\mathrm{MHz})$ \\
\hline \multicolumn{3}{c}{ HCCCN } \\
\hline 30 & 29 & 263792.39 \\
31 & 30 & 272884.78 \\
32 & 31 & 281976.79 \\
33 & 32 & 291068.48 \\
\hline \multicolumn{4}{c}{} \\
& \multicolumn{3}{c}{$\mathbf{( 0 , 0 , 0 , 1 )}$} \\
29 & 28 & $1 e$ & 264439.66 \\
29 & 28 & $1 f$ & 264817.26 \\
30 & 29 & $1 e$ & 273554.24 \\
30 & 29 & $1 f$ & 273944.87 \\
31 & 30 & $1 e$ & 282668.53 \\
31 & 30 & $1 f$ & 283072.00 \\
32 & 31 & $1 e$ & 291782.40 \\
32 & 31 & $1 f$ & 292198.74 \\
\hline
\end{tabular}

\begin{tabular}{llll}
\multicolumn{3}{c}{$(\mathbf{0 , 0 , 0 , 2})$} \\
29 & 28 & $0 e$ & 265403.96 \\
29 & 28 & $2 f$ & 265461.61 \\
29 & 28 & $2 e$ & 265521.99 \\
30 & 29 & $0 e$ & 274547.37 \\
30 & 29 & $2 f$ & 274611.19 \\
30 & 29 & $2 e$ & 274677.94 \\
31 & 30 & $0 e$ & 283689.96 \\
31 & 30 & $2 f$ & 283760.44 \\
31 & 30 & $2 e$ & 283833.92 \\
32 & 31 & $0 e$ & 292831.69 \\
32 & 31 & $2 f$ & 292909.28 \\
32 & 31 & $2 e$ & 292989.79 \\
\hline
\end{tabular}




\begin{tabular}{cccc}
$\mathrm{J}^{\prime}$ & $\mathrm{J}^{\prime \prime}$ & $\ell$ & Observed Frequency $(\mathrm{MHz})$ \\
\hline \multicolumn{3}{c}{$\mathbf{( 0 , 0 , 1 , 0 )}$} \\
\hline 29 & 28 & $1 e$ & 264224.50 \\
29 & 28 & $1 f$ & 264432.01 \\
30 & 29 & $1 e$ & 273331.74 \\
30 & 29 & $1 f$ & 273546.44 \\
31 & 30 & $1 e$ & 282438.62 \\
31 & 30 & $1 f$ & 282660.54 \\
32 & 31 & $1 e$ & 291545.10 \\
32 & 31 & $1 f$ & 291774.09 \\
\hline
\end{tabular}

\begin{tabular}{llll}
\multicolumn{3}{c}{$(\mathbf{0 , 0 , 1 , 1 )}$} \\
\hline 29 & 28 & $0 e$ & 265079.02 \\
29 & 28 & $0 f$ & 265124.65 \\
29 & 28 & $2 f$ & 265216.04 \\
29 & 28 & $2 e$ & 265260.03 \\
30 & 29 & $0 e$ & 274210.26 \\
30 & 29 & $0 f$ & 274260.66 \\
30 & 29 & $2 f$ & 274359.46 \\
30 & 29 & $2 e$ & 274408.23 \\
31 & 30 & $0 e$ & 283340.31 \\
31 & 30 & $0 f$ & 283396.12 \\
31 & 30 & $2 f$ & 283502.58 \\
31 & 30 & $2 e$ & 283556.71 \\
32 & 31 & $0 e$ & 292469.46 \\
32 & 31 & $0 f$ & 292531.11 \\
32 & 31 & $2 f$ & 292645.33 \\
32 & 31 & $2 e$ & 292705.11 \\
\hline & & & \\
32 & 31 & & 263424.67 \\
30 & 29 & & 272504.11 \\
\hline 31 & 30 & & 291583.07 \\
\hline
\end{tabular}




\begin{tabular}{|c|c|c|c|}
\hline $\mathrm{J}^{\prime}$ & $\mathrm{J}^{\prime \prime}$ & $e$ & Observed Frequency $(\mathrm{MHz})$ \\
\hline \multicolumn{4}{|c|}{$(\mathbf{1}, \mathbf{0 , 0}, \mathbf{1})$} \\
\hline 29 & 28 & $1 e$ & 264105.54 \\
\hline 29 & 28 & $1 f$ & 264495.70 \\
\hline 30 & 29 & $1 e$ & 273208.07 \\
\hline 30 & 29 & $1 f$ & 273611.38 \\
\hline 31 & 30 & $1 e$ & 282310.23 \\
\hline 31 & 30 & $1 f$ & 282726.62 \\
\hline 32 & 31 & $1 e$ & 291411.74 \\
\hline 32 & 31 & $1 f$ & Blend \\
\hline \multicolumn{4}{|c|}{$(\mathbf{0 , 1}, \mathbf{0 , 0})$} \\
\hline 29 & 28 & $1 e$ & 263810.70 \\
\hline 29 & 28 & $1 f$ & 263957.70 \\
\hline 30 & 29 & $1 e$ & 272903.99 \\
\hline 30 & 29 & $1 f$ & 273055.75 \\
\hline 31 & 30 & $1 e$ & 281996.27 \\
\hline 31 & 30 & $1 f$ & 282153.38 \\
\hline 32 & 31 & $1 e$ & 291088.41 \\
\hline 32 & 31 & $1 f$ & 291250.69 \\
\hline \multicolumn{4}{|c|}{$(0,0,0,3)$} \\
\hline 29 & 28 & $1 e$ & Blend \\
\hline 29 & 28 & $3 e$ & 266313.28 \\
\hline 29 & 28 & $3 f$ & 266316.17 \\
\hline 29 & 28 & $1 f$ & 266652.00 \\
\hline 30 & 29 & $1 e$ & 275056.96 \\
\hline 30 & 29 & $3 e$ & 275493.74 \\
\hline 30 & 29 & $3 f$ & 275496.82 \\
\hline 30 & 29 & $1 f$ & 275840.78 \\
\hline 31 & 30 & $1 e$ & 284219.50 \\
\hline 31 & 30 & $3 e$ & 284673.61 \\
\hline 31 & 30 & $3 f$ & 284677.65 \\
\hline 31 & 30 & $1 f$ & 285028.44 \\
\hline 32 & 31 & $1 e$ & 293381.47 \\
\hline 32 & 31 & $3 e$ & Blend \\
\hline 32 & 31 & $3 f$ & 293858.01 \\
\hline 32 & 31 & $1 f$ & Blend \\
\hline
\end{tabular}




\begin{tabular}{|c|c|c|c|}
\hline $\mathrm{J}^{\prime}$ & $J^{\prime \prime}$ & $e$ & ed Frequency $(\mathrm{MHz})$ \\
\hline \multicolumn{4}{|c|}{$\mathrm{H}^{13} \mathrm{CCCN}$} \\
\hline 30 & 29 & & 264451.35 \\
\hline 31 & 30 & & 273262.67 \\
\hline 32 & 31 & & Blend \\
\hline 33 & 32 & & 290883.87 \\
\hline \multicolumn{4}{|c|}{$\mathrm{H}^{13} \mathrm{CCCN}(0,0,0,1)$} \\
\hline 30 & 29 & $1 e$ & 265108.45 \\
\hline 30 & 29 & $1 f$ & 265477.71 \\
\hline 31 & 30 & $1 e$ & 273941.40 \\
\hline 31 & 30 & $1 f$ & Blend \\
\hline 32 & 31 & $1 e$ & 282774.04 \\
\hline 32 & 31 & $1 f$ & Blend \\
\hline 33 & 32 & $1 e$ & 291606.19 \\
\hline 33 & 32 & $1 f$ & 292011.93 \\
\hline \multicolumn{4}{|c|}{$\mathrm{HC}^{13} \mathrm{CCN}$} \\
\hline 29 & 28 & & 262673.40 \\
\hline 30 & 29 & & 271727.21 \\
\hline 31 & 30 & & 280780.72 \\
\hline 32 & 31 & & 289833.77 \\
\hline \multicolumn{4}{|c|}{$H C^{13} \mathrm{CCN}(0,0,0,1)$} \\
\hline 29 & 28 & $1 e$ & 263294.54 \\
\hline 29 & 28 & $1 f$ & 263674.24 \\
\hline 30 & 29 & $1 e$ & 272369.68 \\
\hline 30 & 29 & $1 f$ & 272762.51 \\
\hline 31 & 30 & $1 e$ & 281444.49 \\
\hline 31 & 30 & $1 f$ & 281850.04 \\
\hline 32 & 31 & $1 e$ & 290518.78 \\
\hline 32 & 31 & $1 f$ & 290937.60 \\
\hline
\end{tabular}




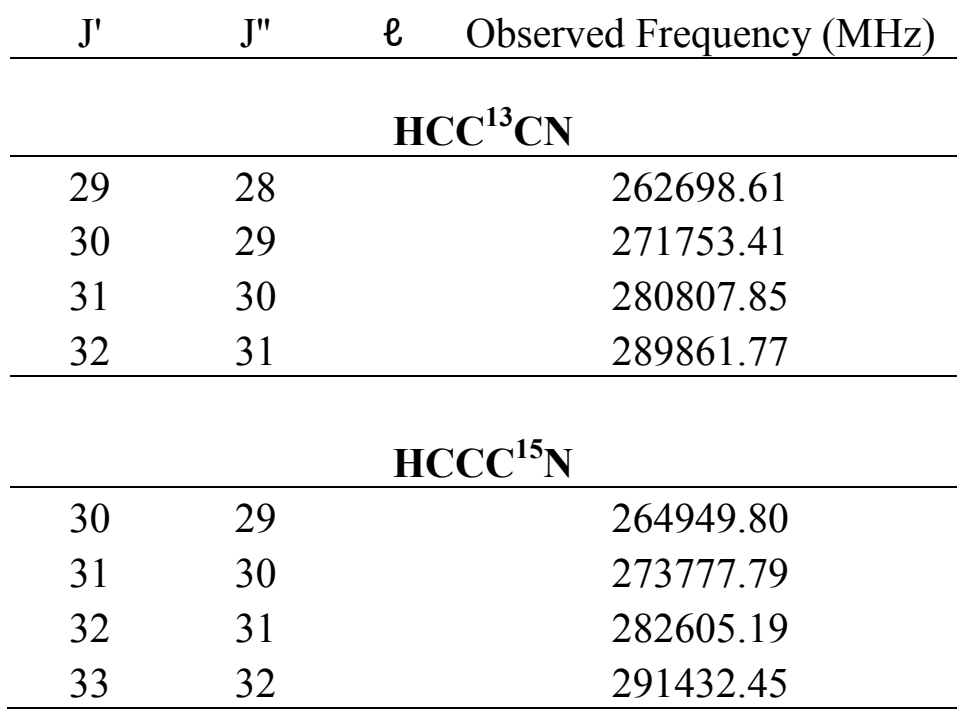




\section{Chapter 6}

\section{A Theoretical and Observational Study of the Formational Pathway of Cyanomethanimine in Sagittarius B2}

\section{Introduction}

Astrochemistry has been an ever-evolving field that in the past decade has seen a significant increase in interest. One motivator for this increased interest is the (arrival of a) new generation of astronomical observatories with sensitive broad bandwidth capabilities at Radio to mid-Infrared wavelengths. The Atacama Large Millimeter Array (ALMA) has just come online and the Karl G. Jansky Very Large Array (VLA) has undergone a significant renovation. Both of these interferometers are able to produce high spatial resolution maps which pair well with single dish observations from observatories including the Robert C. Byrd Green Bank Telescope (GBT). By combining the capabilities of both single dish and interferometric observatories, detailed information on the spatial distributions of tens to hundreds of molecules will become available $^{1}$, enabling observational studies of interstellar formation chemistry. With these instruments, it is now straightforward to determine the spatial distributions of several molecules that are "chemically related" by their formation chemistry, providing tests of proposed chemical reaction mechanisms. This line of inquiry has been published in the past $^{2}$; however, it remains in its infancy. To date, many proposed chemical reaction networks do not account for the spatial distributions of reactant and product species, with few exceptions ${ }^{3}$. As we use this information to begin to understand the chemical reaction pathways by which interstellar molecules form, we will observe dramatic improvements 
in our understanding of star and planet formation, with chemistry tracing the physical history of interstellar regions.

Among the most interesting regions for studying interstellar chemical reaction networks is Sagittarius B2 (Sgr B2). Sgr B2 is a high mass star-forming region located in the Central Molecular Zone (CMZ) of the Galaxy. The region hosts the most diverse set of molecules observed in the interstellar medium (ISM). Sgr B2 has two primary cores of star formation, namely the Main core (Sgr B2(M)) and the North core (Sgr B2(N)), located 45 " to the north. Hot cores are dense $\left(\mathrm{n}>10^{6} \mathrm{~cm}^{-3}\right)$, warm $(100-300 \mathrm{~K})$ cores of active star formation. Surrounding the hot core is a more moderate density, cooler envelope of molecular material harboring numerous molecules including cyanides and oxygenated species ${ }^{4,5}$.

Sgr B2(N) has been targeted by multiple spectral line surveys, including the PRebiotic Interstellar MOlecule Survey (PRIMOS), conducted with the GBT. PRIMOS is the deepest, most frequency-complete centimeter survey of Sgr B2(N) and has already yielded several new molecular detections, including the discovery of interstellar E-cyanomethanimine $(\mathrm{E}-\mathrm{HNCHCN})^{6}$. In that work, Zaleski et al determined that the detected transitions of E-cyanomethanimine have a low rotational temperature of $\mathrm{T}_{\mathrm{ex}}<10 \mathrm{~K}$. This indicates that E-cyanomethanimine is likely not present in the LMH hot core, in which molecules are typically characterized by rotational temperatures of $\mathrm{T}_{\mathrm{ex}}>100 \mathrm{~K}$ 
The interstellar detection of E-cyanomethanimine was made by comparing the PRIMOS spectrum of Sgr B2(N) directly to laboratory spectra obtained using chirped pulse-Fourier transform microwave (CP-FTMW) spectroscopy. In this method known as the broadband reaction screening process, cyanomethanimine was synthesized in a discharge of $\mathrm{NH}_{3}$ and $\mathrm{CH}_{3} \mathrm{CN}$ and monitored by CP-FTMW spectroscopy between 6-18.5 GHz , 25-40 GHz, and 40-50 GHz. Measurements were also taken with the FTMW spectrometer at the Harvard-Smithsonian Center for Astrophysics to refine the hyperfine components. In addition to E-cyanomethanimine, both additional isomers of cyanomethanimine - the Z-isomer and the $\mathrm{N}$-isomer - were detected in the laboratory spectra. The Z-isomer is the lowest energy isomer but has the weakest dipole moment, whereas the $\mathrm{N}$-isomer $\left(\mathrm{H}_{2} \mathrm{C}=\mathrm{N}-\mathrm{CN}\right)$ is the highest energy isomer but has the highest dipole moment and is higher in energy above the Z-isomer by approximately $10 \mathrm{kcal} \mathrm{mol}{ }^{-1}$. The PRIMOS spectrum additionally provides some evidence for the highest energy isomer $(\mathrm{N}-)$ in $\operatorname{Sgr} \mathrm{B} 2(\mathrm{~N})^{6}$. The three isomers of cyanomethanimine provide an excellent opportunity to study interstellar formation chemistry.

Conditions in the ISM are very different from terrestrial conditions, with very low densities and in most material in giant molecular clouds (GMC's), low temperatures. As a result, unless there is an astrophysical mechanism driving a reaction, it is unlikely to occur if there is an activation barrier. The mechanisms that have been proposed for the formation of cyanomethanimine, have all had prohibitively high activation energy barriers and cannot explain the existence of the three isomers of cyanomethanimine. This work presents a proposed barrierless chemical reaction pathway for forming all three 
isomers of cyanomethanimine in the ISM. The pathway has been developed based on the work done by Balucani et al. ${ }^{7}$ in which the cyanide radical $\left({ }^{\circ} \mathrm{CN}\right)$ can react with olefins $(\mathrm{C}=\mathrm{C})$ through a barrier-less reaction. While this type of reaction has not been tested on imines, it is plausible to consider the reaction of methanimine $\left(\mathrm{CH}_{2} \mathrm{NH}\right)$ and ${ }^{\circ} \mathrm{CN}$, both abundant molecules in $\mathrm{Sgr} B 2(\mathrm{~N})^{8,9}$. In this reaction ${ }^{\circ} \mathrm{CN}$ could react at either the $\mathrm{C}$ or the $\mathrm{N}$ to produce all three isomers of cyanomethanimine, shown in Eq. 6.1.

$$
\mathrm{CH}_{2} \mathrm{CN}+\mathrm{CN} \rightarrow \mathrm{H}_{2} \mathrm{C}_{2} \mathrm{~N}_{2}+\mathrm{H}
$$

The three conformers can be seen in Fig. 6.1. Thus a study of this plausible reaction mechanism has been undertaken via quantum chemical calculations and exploratory molecular mapping with the VLA.

\section{Experimental Details}

a) Ab initio calculations

All ab initio calculations were performed using Gaussian ' $09^{10}$. All structures were optimized using the MP2/6-311++g(d,p) level of theory and their relative energies were corrected with harmonic zero point vibrational energy corrections. The transition states were found using the QST3 STQN method. 


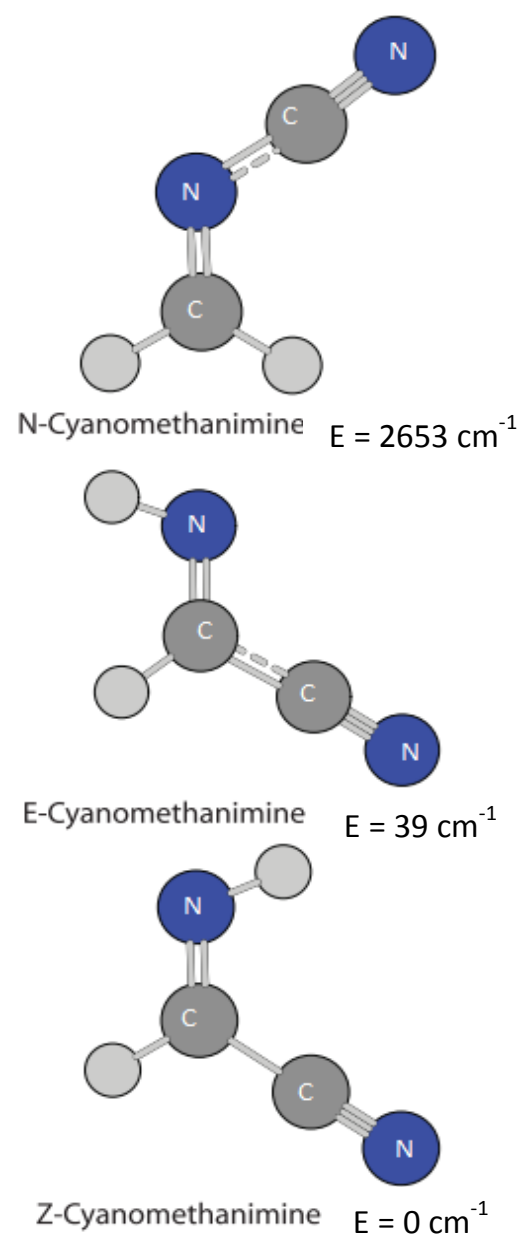

Figure 6.1. The three isomers of cyanomethanimine and their zero-point energy (ZPE) corrected relative energies. 
b) Observational details

We conducted all observations with the Karl G. Jansky Very Large Array (VLA) towards a single pointing centered on the Large Molecule Heimat (LMH) at $(\mathrm{J} 2000)(\alpha, \delta)=$ (17h47m19.8s, $\left.-28^{\circ} 22^{\prime} 17^{\prime \prime}\right)$. We used VLA capabilities offered through the Resident Shared Risk Observing program in May 2012 to observe the $22_{02}-1_{01}$ transition of E-cyanomethanimine at $19142 \mathrm{MHz}$ and the $1-0$ transition of $\mathrm{CH}_{2} \mathrm{CN}$ at $20125 \mathrm{MHz}$ with $\sim 1 \mathrm{~km} \mathrm{~s}^{-1}$ channel resolution as part of a larger observational campaign. Observations were conducted in good weather during the move from the $\mathrm{CnB}$ to the $\mathrm{B}$ array configuration, resulting in a $1 \times 0.9$ arcsecond beam with natural weighting at $19 \mathrm{GHz}$. In total 4 hours of on-source observing was completed in 6 hours of total observing. The quasar source $1331+305$ (3C286) was used to calibrate the bandpass and absolute flux. We observed a cycle of 4 minutes on the complex gain calibrator (J17132658), 15 minutes on source and updated the telescope pointing (towards J1743-0350) approximately every hour.

We observed the $1_{11}-2_{02}$ and $3_{03}-2_{12}$ transitions of $\mathrm{CH}_{2} \mathrm{NH}$ at 33704 and $35065 \mathrm{MHz}$ respectively in a single 2 hour observation conducted on 28 July 2013 in the C array configuration, resulting in a $2.2 \times 0.8$ " beam with natural weighting. $3 \mathrm{C} 286$ was again used for bandpass and absolute flux calibration. The source and complex gain calibrator (J1833-2103) were observed in cycles of 1 minute on the complex gain calibrator, 3 minutes on source, and telescope pointing updates were determined towards the gain calibrator approximately every hour. A total of 1 hour of on-source observing was completed. 
All data were reduced and calibrated using standard techniques with the CASA software package $^{11}$, and phase only self calibration was applied, with phase solutions determined using the continuum towards Sgr B2. The data cubes and continuum images were produced using the Cotton-Schwab CLEAN algorithm ${ }^{12}$ with briggs weighting to suppress sidelobes. All images were then convolved to a $2 \times 2$ "Gaussian beam for comparison.

\section{Results and Discussion}

The quantum chemistry calculations (Fig. 6.2) indicate that the reaction between $\mathrm{CH}_{2} \mathrm{NH}$ and ${ }^{\circ} \mathrm{CN}$ is an exothermic, barrier-less reaction for all three products. The pathways to form both the E- and Z- isomers go through the same $\mathrm{sp}^{3}$ transition state that is $-25.6 \mathrm{kcal} \mathrm{mol}^{-1}$ lower than the reactants. This can be considered a barrier-less reaction, as the transition state corresponds to a maximum in the torsional potential of the $\mathrm{H}-\mathrm{C}-\mathrm{N}$ $\mathrm{H}$ dihedral. The minima in this potential correspond to intermediate states leading to the formation of the E- and Z- isomers, which are differentiated solely by the direction of the $\mathrm{N}-\mathrm{H}$ bond. While the relative energies favor the Z-isomer, the two isomers are close in energy differing by only $0.2 \mathrm{kcal} \mathrm{mol}^{-1}$. For the reaction in which the $\mathrm{N}$-isomer is formed, the transition state is $-29.7 \mathrm{kcal} \mathrm{mol}^{-1}$ relative to the reactants, but this isomer is the highest in energy when compared to the Z- and E- isomers. Because the ab initio results indicate that the formation of all three of the isomers is exothermic and proceed without a barrier, it is plausible that this reaction could produce the three isomers in the ISM. 


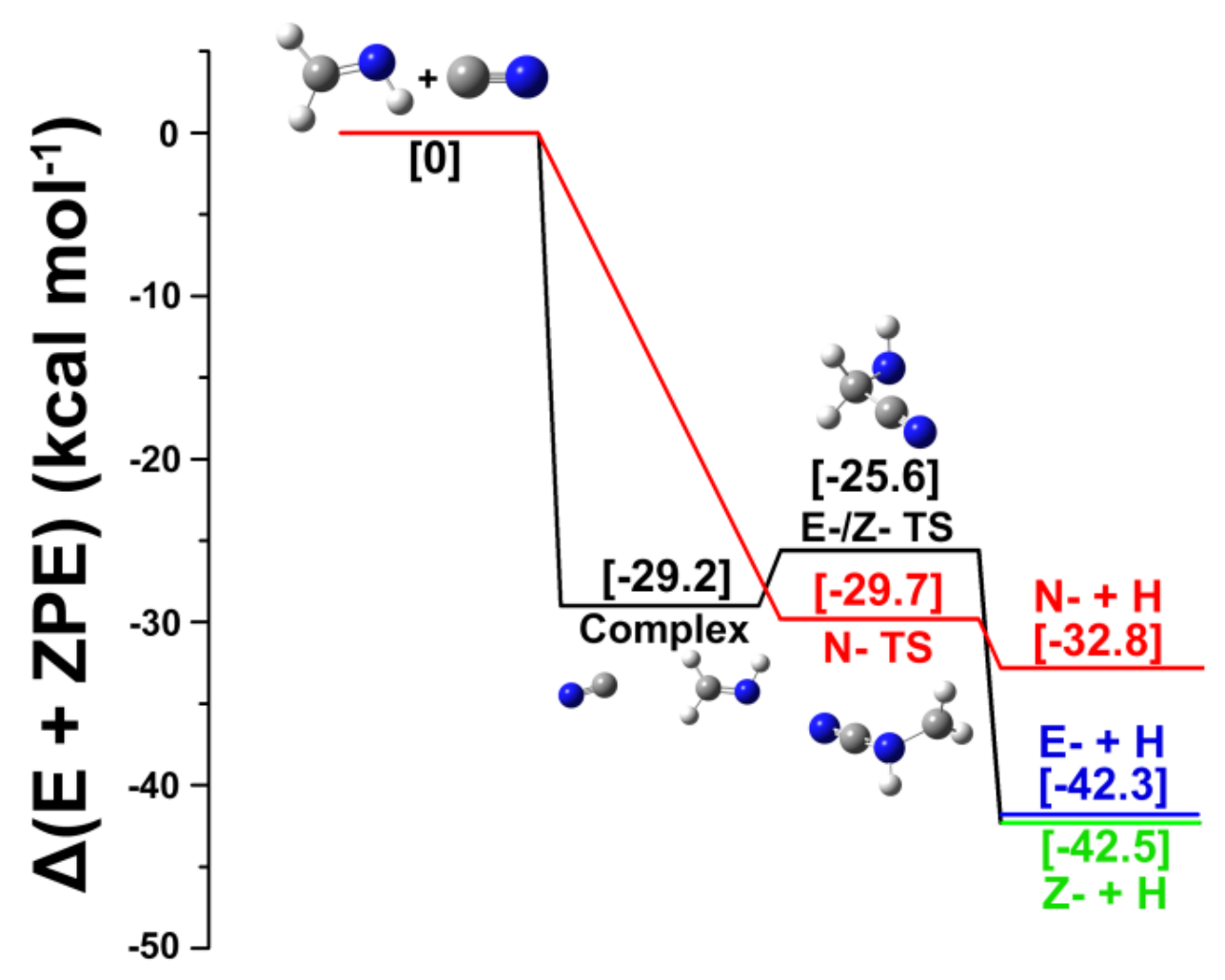

Figure 6.2. Energetic profile of proposed neutral-radical reaction between $\mathrm{CH}_{2} \mathrm{NH}$ and ${ }^{\circ} \mathrm{CN}$ to form the three isomers of cyanomethanimine. The structures were optimized with the MP2/6-311++g(d,p) level of theory. The energies were corrected to account for zeropoint vibrational energy. The black line indicates the pathway for the formation of both the E- and Z- isomers of cyanomethanimine, while the red line shows the formation pathway for the $\mathrm{N}$ - isomer. The transition states were calculated using the QST3 STQN method. 
The radio continuum of $\mathrm{Sgr} \mathrm{B} 2(\mathrm{~N})$ at $35 \mathrm{GHz}$ is shown in Fig. 6.3 with the different structures labeled according to the naming convention of Gaume et al. ${ }^{13}$. The continuum emission is generated by free-free scattering in the labeled shell- and coreshaped HII regions. The hot core, otherwise referred to as the Large Molecular Heimat $(\mathrm{LMH})$, is a doughnut shaped region that encircles the $\mathrm{K} 2$ and $\mathrm{K} 3$ regions. The labeled regions represent shells or compact HII regions. In order to test the proposed reaction, the spatial distributions were studied and can be seen in Fig. 6.4. The spatial distributions of $\mathrm{CH}_{2} \mathrm{NH}$ (pink), ${ }^{\circ} \mathrm{CH}_{2} \mathrm{CN}$ (green), and E-HNCHCN (blue) in Sgr B2(N) (top panel and Sgr B2(M) (bottom panel) are shown overlaid on the $35 \mathrm{GHz}$ continuum image. While $\mathrm{CH}_{2} \mathrm{NH}$ has a wide spread spatial distribution in Sgr B2(N), E-HNCHCN and ${ }^{\circ} \mathrm{CH}_{2} \mathrm{CN}$ are more localized. All three are co-spatial in the K5 and K6 shells. In Sgr B2(M), the spatial distributions are very similar with a high spatial overlap. E-HNCHCN is present where both of the reactants are observed. This spatial overlap is fully consistent with the proposed mechanism and help to validate the proposed reaction in Eq. 6.1. The other thing to note is that the spatial distribution of E-HNCHCN in Sgr B2(N) is not found in the $\mathrm{LMH}$ as seen in Fig. 6.4. As stated previously it resides predominantly in the K5 and K6 HII shells. Thus the chemistry leading to a complex, prebiotic molecule is most likely occurring outside of the hot core region; the idea that large molecule formation is mainly a hot core phenomenon may not be valid in this case.

While optimally, it would possible to compare the distributions of product and reactant species directly, no high spatial resolution map of the cyano radical $\left({ }^{\circ} \mathrm{CN}\right)$ is available, although the species has been detected in $\mathrm{Sgr} \mathrm{B} 2^{9}$. The cyanomethyl radical 


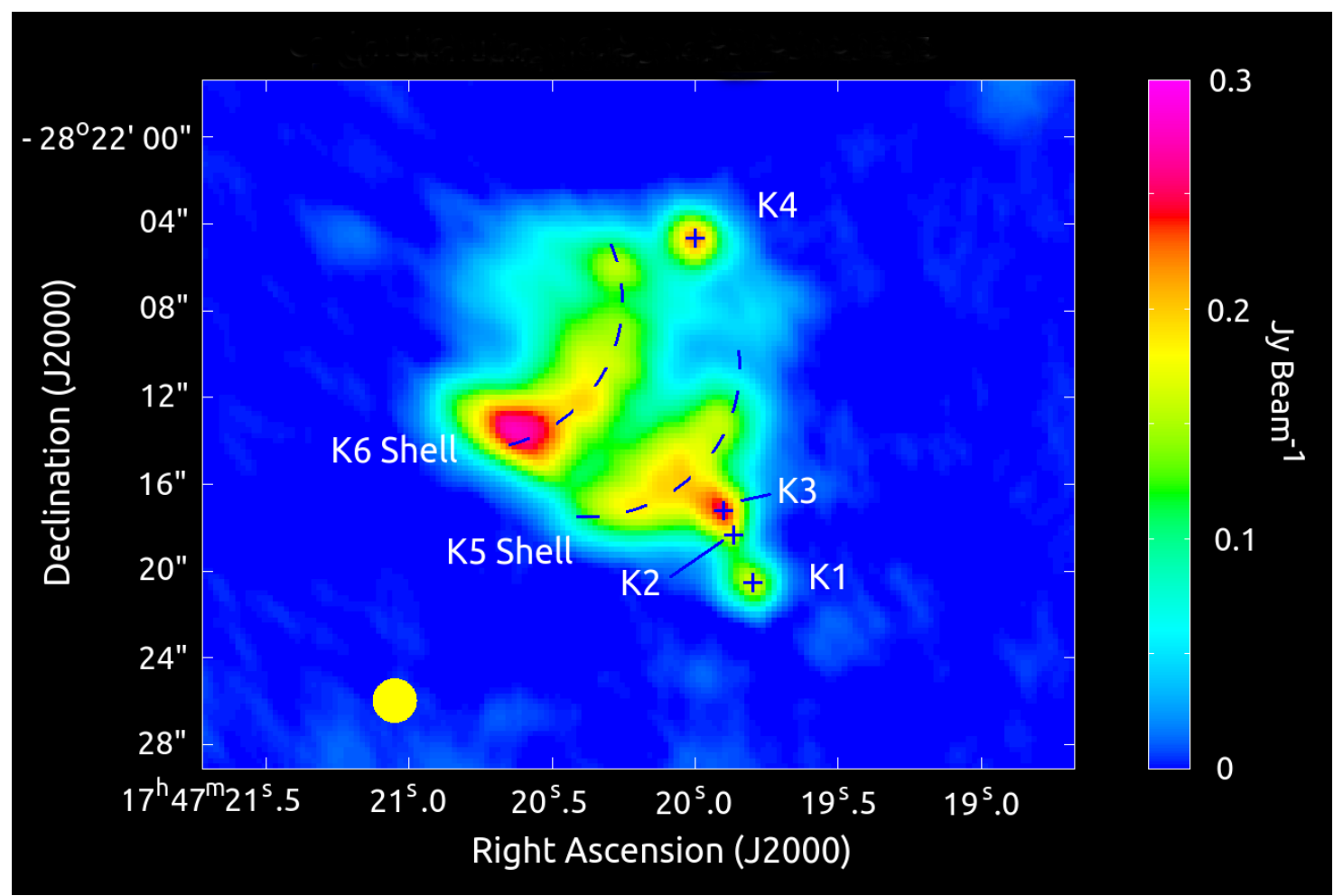

Figure 6.3. Continuum map of Sgr B2(N) taken at $35 \mathrm{GHz}$ with the VLA. This map highlights the compact and shell HII regions. The naming convention was taken from Gaume et al. ${ }^{13}$. 


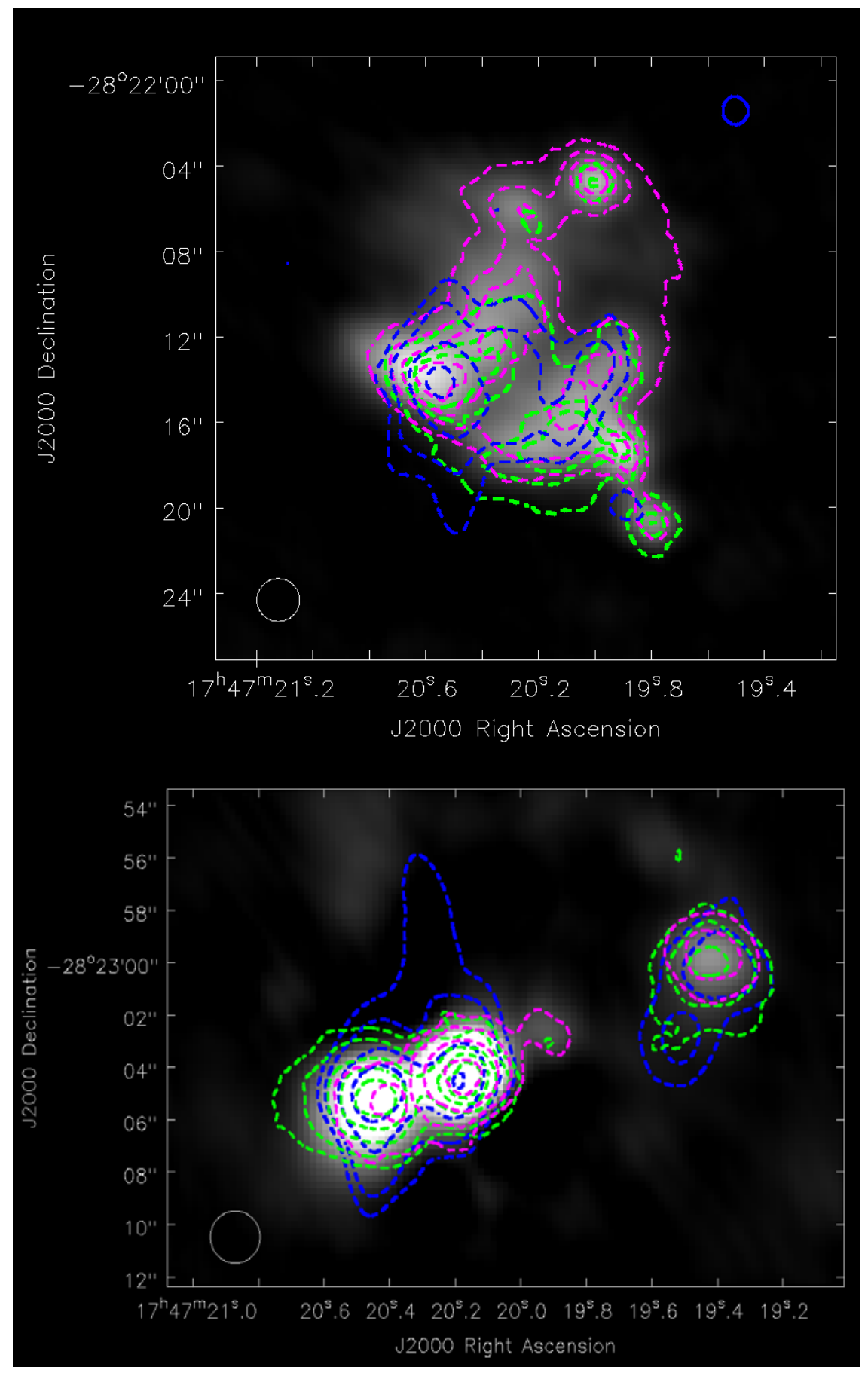

Figure 6.4. Spatial distributions of methanimine (pink), E-cyanomethanimine (blue) and - $\mathrm{CH}_{2} \mathrm{CN}$ (green) all in absorption; the distributions have been overlaid on the continuum image at $35 \mathrm{GHz}$. The top panel shows Sgr B2(N) where the distributions are co-spatial in the $\mathrm{K} 5$ and $\mathrm{K} 6$ shells, while in the bottom the co-spatial distributions can be seen for Sgr B2(M). 
$\left({ }^{\circ} \mathrm{CH}_{2} \mathrm{CN}\right)$, for which low frequency transitions fall within the VLA frequency coverage, was instead used as a tracer for ${ }^{\circ} \mathrm{CN}$. While no experimental study on the formation of ${ }^{\circ} \mathrm{CN}$ and ${ }^{\circ} \mathrm{CH}_{2} \mathrm{CN}$ in astronomically relevant environments has been published, they could likely form under similar conditions by dissociating a hydrogen atom from the abundant molecules $\mathrm{HCN}^{14}$ and $\mathrm{CH}_{3} \mathrm{CN}$. For example, ultraviolet (UV) radiation is sufficiently energetic to remove a hydrogen bond. The molecular gas on the interface of the HII regions should have an enhanced UV radiation field creating a photo-dissociation region (PDR). With the very complex structure of the ionized, neutral, and molecular gas in Sgr B2, it is difficult to say conclusively that the molecular gas probed by the VLA data is a PDR. However, the very low $(<10 \mathrm{~K})$ excitation temperatures of observed imines ${ }^{6,15}$ are entirely inconsistent with the kinetic temperatures of molecular gas throughout the $\mathrm{CMZ}^{16}$, indicating that the gas is diffuse. This is consistent with the gas being a PDR. Alternatively X-ray irradiation of $\mathrm{HCN}$ and $\mathrm{CH}_{3} \mathrm{CN}$ might accomplish the hydrogen dissociation. Sgr B2 is a known X-ray reflection nebula with a large X-ray flux through the molecular material ${ }^{17,18}$. Regardless of the exact scenario, the distributions of ${ }^{\circ} \mathrm{CN}$ and ${ }^{\circ} \mathrm{CH}_{2} \mathrm{CN}$ likely correspond quite closely, enabling the use of ${ }^{\circ} \mathrm{CH}_{2} \mathrm{CN}$ to trace ${ }^{\circ} \mathrm{CN}$.

Observations of the ${ }^{\circ} \mathrm{CN}$ radical, along with observations of the $\mathrm{CH}_{2} \mathrm{NH}$ and E-HNCHCN at the same frequency, need to be obtained in order to completely validate this theory. While the ${ }^{\circ} \mathrm{CH}_{2} \mathrm{CN}$ is a good tracer, it is important that direct comparison to the cyano radical distribution is made once observations on a high resolution interferometer such as ALMA become available. Methanimine has been shown to be abundant at higher frequencies ${ }^{8}$, and it is uncertain if E-HNCHCN would be detectable. 
At the higher frequencies of the $\mathrm{J}=1-0$ transition of ${ }^{\circ} \mathrm{CN}, \mathrm{E}-\mathrm{HNCHCN}$ may be difficult to detect without the strong continuum observed at low frequency if the material is indeed exclusively distributed in low density material with a high UV flux. However, if E-HNCHCN is present in the hot core, this would demonstrate that the low frequency observations of transitions between low (sub-20K) energy states produced significant bias towards detecting the molecules in absorption against the shell-shaped HII regions.

This method of inquiry about the chemical reaction networks of the ISM will become a key component of the analysis of a source as more high resolution interferometric data becomes available. The spatial distributions of a source's molecular components offer information about the reaction networks that may be occurring in that source. While it is possible for astrophysical phenomena to influence these distributions, these types of formation pathways and spatial information should be incorporated into existing chemical models.

\section{Conclusions}

After the discovery of E-cyanomethanimine through broadband reaction screening, paired with the PRIMOS data set from the GBT, interferometric data has been used to support a proposed formation mechanism in the ISM. Data from the VLA was used to map the spatial distributions of $\mathrm{CH}_{2} \mathrm{NH}, \mathrm{HNCHCN}$, and ${ }^{\circ} \mathrm{CH}_{2} \mathrm{CN}$ (from which the distribution of ${ }^{\circ} \mathrm{CN}$ was inferred). With ${ }^{\circ} \mathrm{CH}_{2} \mathrm{CN}$ used to trace ${ }^{\circ} \mathrm{CN}$, the product and reactant species in the proposed reaction are determined to be cospatial in Sgr B2(N) and 
(M), providing evidence that the proposed reaction may be responsible for the formation of E-cyanomethanimine. Further, it shows that this chemistry is occurring outside of the hot core, in less dense material which may be interacting with expanding HII regions dynamically (i.e. shocks) or radiatively (i.e. UV radiation). High spatial resolution observations of the cyano radical will be important for validating this conclusion.

By coordinating multiple techniques-namely single dish radio astronomy, interferometry, CP-FTMW, and ab initio calculations - a pre-biotic molecule was detected in the ISM, and its reaction mechanism was explored. This method of inquiry, comparing the distributions of reactant and product molecules, can contribute significantly towards constraining interstellar chemical reaction networks as high resolution interferometric data becomes increasingly available. While observational selection effects must be carefully considered, the spatial information should be incorporated into existing chemical models. Methods such as this will help to further our understanding of the chemistry occurring in astronomical sources. 


\section{Chapter 6 References}

(1) Herbst, E. Astrophys. Space Sci. 2008, 313, 129-134.

(2) Neill, J. L.; Steber, A. L.; Muckle, M. T.; Zaleski, D. P.; Lattanzi, V.; Spezzano, S.; McCarthy, M. C.; Remijan, A. J.; Friedel, D. N.; Widicus Weaver, S. L.; Pate, B. H. J. Phys. Chem. A 2011, 115, 6472-6480.

(3) Aikawa, Y.; Wakelam, V.; Garrod, R. T.; Herbst, E. Astrophys. J. 2008, 674, 984.

(4) Jones, P. A.; Cunningham, M. R.; Godfrey, P. D.; Cragg, D. M. Mon. Not. R. Astron. Soc. 2007, 374, 579-589.

(5) Jones, P. A.; Burton, M. G.; Tothill, N. F. H.; Cunningham, M. R. Mon. Not. R. Astron. Soc. 2011, 411, 2293-2310.

(6) Zaleski, D. P.; Seifert, N. A.; Steber, A. L.; Muckle, M. T.; Loomis, R. A.; Corby, J. F.; Oscar Martinez, J.; Crabtree, K. N.; Jewell, P. R.; Hollis, J. M.; Lovas, F. J.; Vasquez, D.; Nyiramahirwe, J.; Sciortino, N.; Johnson, K.; McCarthy, M. C.; Remijan, A. J.; Pate, B. H. Astrophys. J. Lett. 2013, 765, L10.

(7) Balucani, N.; Asvany, O.; Huang, L. C. L.; Lee, Y. T.; Kaiser, R. I.; Osamura, Y.; Bettinger, H. F. Astrophys. J. 2000, 545, 892-906.

(8) Halfen, D. T.; Ziurys, L. M.; Brünken, S.; Gottlieb, C. A.; McCarthy, M. C.; Thaddeus, P. Astrophys. J. Lett. 2009, 702, L124.

(9) Nummelin, A.; Bergman, P.; Hjalmarson, Å.; Friberg, P.; Irvine, W. M.; Millar, T. J.; Ohishi, M.; Saito, S. Astrophys. J. Suppl. Ser. 2000, 128, 213-243.

(10) Frisch, M. J.; Trucks, G. W.; Schlegel, H. B.; Scuseria, G. E.; Robb, M. A.; Cheeseman, J. R.; Scalmani, G.; Barone, V.; Mennucci, B.; Petersson, G. A.; 
Nakatsuji, H.; Caricato, M.; Li, X.; Hratchian, H. P.; Izmaylov, A.; Bloino, J.; Zheng, G. Gaussian 09; Gaussian, Inc.: Wallingford, CT, 2009.

(11) Common Astronomy Software Application; National Radio Astronomy Observatory.

(12) Schwab, F. R. Astron. J. 1984, 89, 1076-1081.

(13) Gaume, R. A.; Claussen, M. J.; de Pree, C. G.; Goss, W. M.; Mehringer, D. M. Astrophys. J. 1995, 449, 663.

(14) Guo, J.; Eng, R.; Carrington, T.; Filseth, S. V. J. Chem. Phys. 2000, 112, 89048909.

(15) Loomis, R. A.; Zaleski, D. P.; Steber, A. L.; Neill, J. L.; Muckle, M. T.; Harris, B. J.; Hollis, J. M.; Jewell, P. R.; Lattanzi, V.; Lovas, F. J.; Oscar Martinez, J.; McCarthy, M. C.; Remijan, A. J.; Pate, B. H.; Corby, J. F. Astrophys. J. Lett. 2013, 765, L9.

(16) Mills, E. A. Extreme Gas Properties in the Cntral 10 Parsecs, 2014.

(17) Lis, D. C.; Schilke, P.; Bergin, E. A.; Emprechtinger, M. R. Soc. Lond. Philos. Trans. Ser. A 2012, 370, 5162-5173.

(18) Terrier, R.; Ponti, G.; Bélanger, G.; Decourchelle, A.; Tatischeff, V.; Goldwurm, A.; Trap, G.; Morris, M. R.; Warwick, R. Astrophys. J. 2010, 719, 143-150. 


\section{Chapter 7}

\section{The Development of the Spatial Distribution Screening Method and Its Applications} to ALMA Datasets

\section{Introduction}

The past approaches to astronomical molecular detections have consisted of acquiring the laboratory rotational spectra and comparing them to astronomical spectra from desired sources. Over the years, work has been done to increase the speed of laboratory spectroscopic measurements and assignments ${ }^{1-6}$. As discussed in Chapter 5, a bottleneck in the flow of spectral information has occurred due to the long spectral analysis and accumulation times. Instruments such as the FASSST instrument ${ }^{7,8}$ and the chirped pulse-Fourier transform millimeter wave (CP-FTmm-wave) spectrometer ${ }^{9}$ have been developed to help tackle this problem. Both techniques have developed ways in which to acquire spectra in a short period of time, sometimes as a little as 1 second, and have automated the spectral acquisition and, to a certain extent, analysis. The databases $^{10-12}$ discussed in Chapter 5 have all been created to catalog the known rotational spectral information to aid with the identification of interstellar species. Throughout the years, the detections have totaled almost 200 molecules $^{13}$; however, many of these molecules have been simple molecules (diatomic and triatomic molecules) as complex organic molecules can be hard to identify. There are two reasons for this: one being the abundance of these more complex molecules maybe lower and the other is that the transitions for molecules with small dipole moments will be weaker. The first 
branched alkyl molecule was just detected ${ }^{14}$, indicating that these complex organic molecules are present and the right tools need to be utilized. Unfortunately there has been almost no systematic approach with which the interstellar molecules were identified. With these detections and more sophisticated interferometric maps, information about the distribution of these molecules has become known. In star forming regions like the Orion Kleinmann-Low nebula (Orion KL), the chemistry that occurs in these dense molecular clouds produces molecules from all of the chemical "families". These families include amines, amides, cyanides, alkanes, aldehydes, among others. As the region evolves and reactive chemistry takes place through radical-radical reactions, neutral-radical reactions, and neutral-ion reactions ${ }^{15}$, distinct chemical regions emerge ${ }^{16,17}$. The formation of these different regions dictates the chemistry that is occurring in the source.

In the case of Orion KL, the nitrogen bearing family is found in the warmer hot core region ${ }^{16}$, while oxygen bearing species are found to be more centralized in the compact ridge ${ }^{17}$. Because of this spatial differentiation, one would imagine that the chemistry taking place in these regions would occur between molecules that reside in the same place. Not only will they reside in the same region, but in order to react, the molecules should be co-spatial. Thus it is important to fully characterize each local environment not only by its molecular composition, but also by the spatial distribution of each of the constituents. Characterization (both physical and chemical) of these regions will be important in order to understand the chemistry that is occurring in these regions as well as the complex network of reactions taking place. 
The Atacama Large Millimeter Array (ALMA), which has been described previously in this thesis, is one of the ways to obtain the chemical spatial distributions at mm-wave frequencies. ALMA has the ability to produce images with spatial resolution as small as 10 milliarcseconds and has unprecedented sensitivity. It began taking science verification (SV) data in 2012, and one of its first measurements was a Band 6 survey of Orion KL. The dataset included not only broadband rotational spectra, but also spatial distributions. All of this allows for unbiased spectral surveys. From these surveys, the chemical composition and physical environments of sources like Orion KL can be characterized. Because of the large amounts of data, new and efficient analysis tools are needed. The previous method of picking a molecule based on chemical reasoning, obtaining the rotational spectrum, and then acquiring the astronomical spectrum to determine if the molecule is present is inefficient and time-consuming. The density of lines, along with the number of spectra that are generated with each data set from ALMA would require months if not years to analyze with this technique. Thus, a method that works in a similar fashion to the successful broadband reaction screening process ${ }^{18,19}$ discussed in Chapter 6 was developed. The aim of this technique is to utilize the spatial and spectral information that is being produced by ALMA to test the feasibility of proposed reaction mechanisms, and narrow the search region for a potentially less abundant molecule. In this process a viable formation pathway is set forth for a molecule of interest. The spatial distributions of the reactants are then studied to see if they reside in the same regions. If this is the case, and the kinematic profiles of the two molecules looks the same, it is possible that a reaction could be occurring in that area, and thus we 
use the spectral information to look for its presence. This is called the spatial distribution screening process, and it was used to try and identify less prevalent molecules in Orion KL that have been found in other molecular clouds such as Sgr B2.

\section{Experimental}

The data used in this study was part of ALMA science verification Band 6 survey taken on January 20, 2012. The publicly available dataset, which covered 214-247 GHz, used 16 antennas that had a diameter of $12 \mathrm{~m}$. The array configuration for the observation provided an angular resolution of about $1.5 " \mathrm{x} 0.75$ ". The data has a spectral resolution of $488 \mathrm{kHz}\left(0.6 \mathrm{~km} \mathrm{~s}^{-1}\right)$, and each of the spectral windows received 5 minutes of signal integration. The phase center coordinates were $\alpha_{\mathrm{J} 2000}=5 \mathrm{~h} 35 \mathrm{~m} 14 \mathrm{~s} .35$ and $\delta_{\mathrm{J} 2000}=$ $-05^{\circ} 22^{\prime} 35^{\prime}$. The phase calibrator was the quasar J0607-085 and the absolute flux calibrator was Callisto. The fully calibrated measurement set is publicly available at https://almascience.nrao.edu/alma-data/science-verification.

The full data set was deconvolved using the Common Astronomy Software Application package ${ }^{20}$ with the CLEAN algorithm. The continuum was subtracted from each of the 19 windows from line-free spectral channels, and a general deconvolution of each spectral window was done. Seven regions were identified to contain most of the flux. The CLEAN algorithm was implemented with Natural weighting and a pixel size of 0.4". The continuum image at $230.2 \mathrm{GHz}$ was created by using the Briggs weighting with a robust parameter of 0.5 for the deconvolution. 
The methodology used for the spatial distribution screening process was as follows. The spatial distributions for the two suspected reactants were mapped, after which the two maps were overlaid. A pixel from each region that had the best overlap, the highest intensities for both reactants, was chosen. The spectrum from each of the chosen pixels was then plotted against a simulation of the product molecule. The velocity used was dependent upon the reactants velocities in that region as well as the temperatures. These parameters were based on those found by Crockett et al. ${ }^{21}$.

\section{Source information}

As Orion KL has been studied extensively, several different chemical and physical regions have been identified. Fig. 7.1 shows the continuum image of Orion KL at 230.2 GHz, with the hot core, compact ridge, North West region (NW), IRc2, IRc7, and Source I labeled. The Becklin-Neugebauer (BN) object has been labeled for as a reference point. The hot core consists of two regions, the hot core and the southern hot core $^{21}$, and typically has the highest rotational temperatures and amount of complex molecular species. The molecular rotational temperatures of the compact ridge are similar to the hot core; however radial velocity of the material in the compact ridge is between 8$10 \mathrm{~km} / \mathrm{s}$ as opposed to the hot core in which the average velocity is $6 \mathrm{~km} / \mathrm{s}$. The NW region is not typically defined in the past surveys; however, the nomenclature MF4 was used for this region in the work done by Favre and co-workers ${ }^{22}$. The temperatures in this region are roughly the same as the compact ridge, as are the molecular velocities. The 


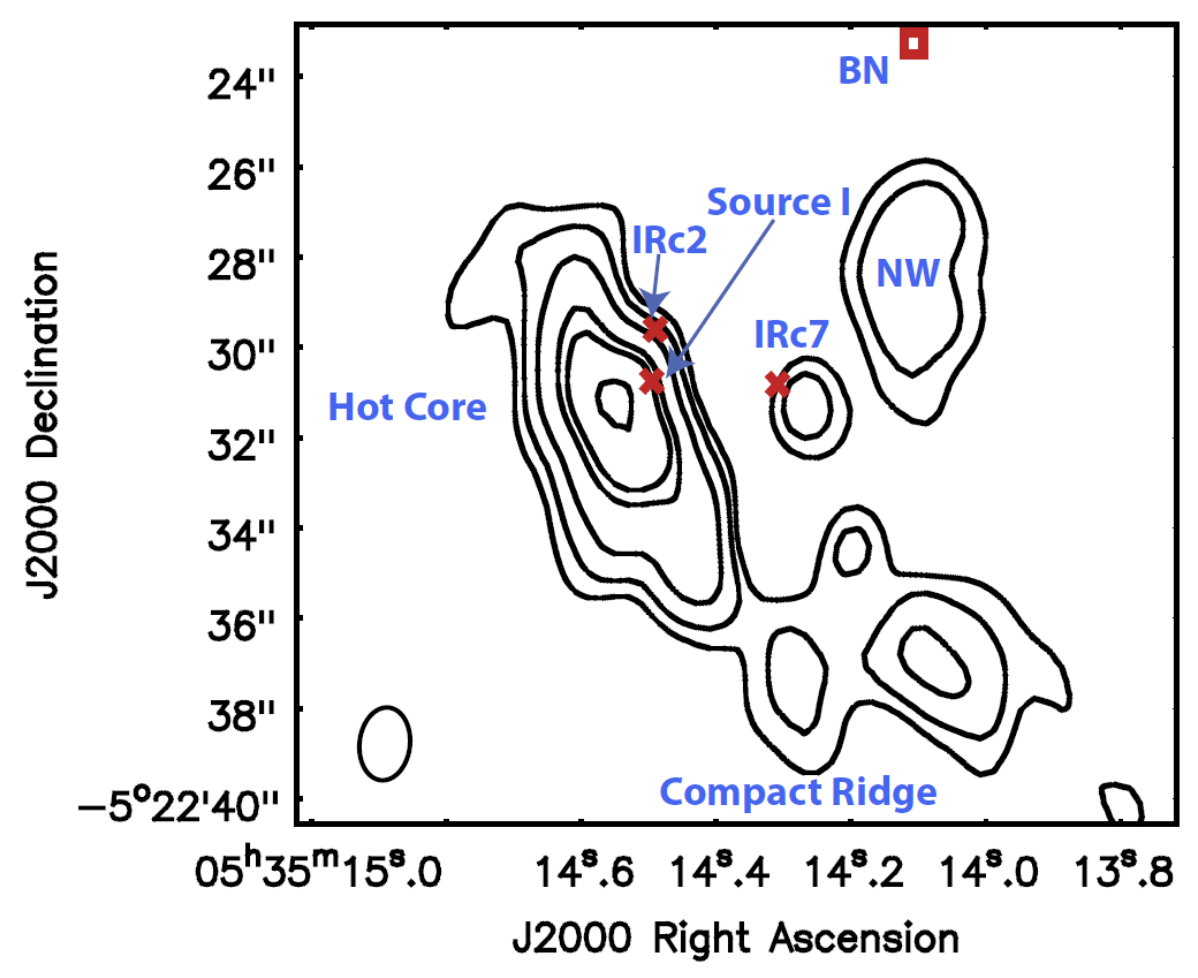

Figure 7.1 Continuum image of Orion $\mathrm{KL}$ at $230.9 \mathrm{GHz}$. The contours correspond to $0.0667,0.1334,0.2668,0.5336,0.6670,1.0005 \mathrm{Jy}_{\text {beam }}{ }^{-1}$. Features of note have been labeled on the continuum image, with the hot core and compact ridge being labeled. The red box in the upper right hand corner signifies the position of $\mathrm{BN}$. 
extended ridge (not labeled on Fig. 7.1) is the cooler and less dense material surrounding these two regions. A depiction of the morphology can also be seen in Crockett et al. ${ }^{21}$. IRc2, IRc7 and BN are luminous sources at infrared wavelengths and the radio source $\mathrm{I}$ is an embedded massive protostar. They all influence the structure of the Orion molecular cloud (OMC-1) $)^{23}$.

\section{Results}

An integrated intensity map, which shows the spatial intensity of the transition across all of this velocity channels, for 9 molecules from three distinct chemical families is shown in Fig. 7.2 to illustrate the distributions of the chemical "families". The top panel shows 3 N-bearing molecules; ethyl cyanide, vinyl cyanide, and ${ }^{13} \mathrm{C}$ methyl cyanide. Ethyl cyanide and vinyl cyanide reside mostly in the hot core, while methyl cyanide resides in the hot core and the southern hot core. The next row of molecules is the oxygen bearing molecules. The spatial distributions of methanol, methyl formate, and dimethyl ether show distributions that are in the southern hot core, compact ridge, and the NW. Methanol is the only molecule that is seen in the hot core as well. The morphologies of S-bearing species are more ubiquitous, with their peak intensities occurring in the hot core. In areas in which overlap occurs between the different families, it is plausible that chemistry is occurring between reactive species of each family. Thus it is likely that S-bearing species could be reacting with not only O-bearing species, but also N-bearing species as their distribution is wide spread across Orion KL. 

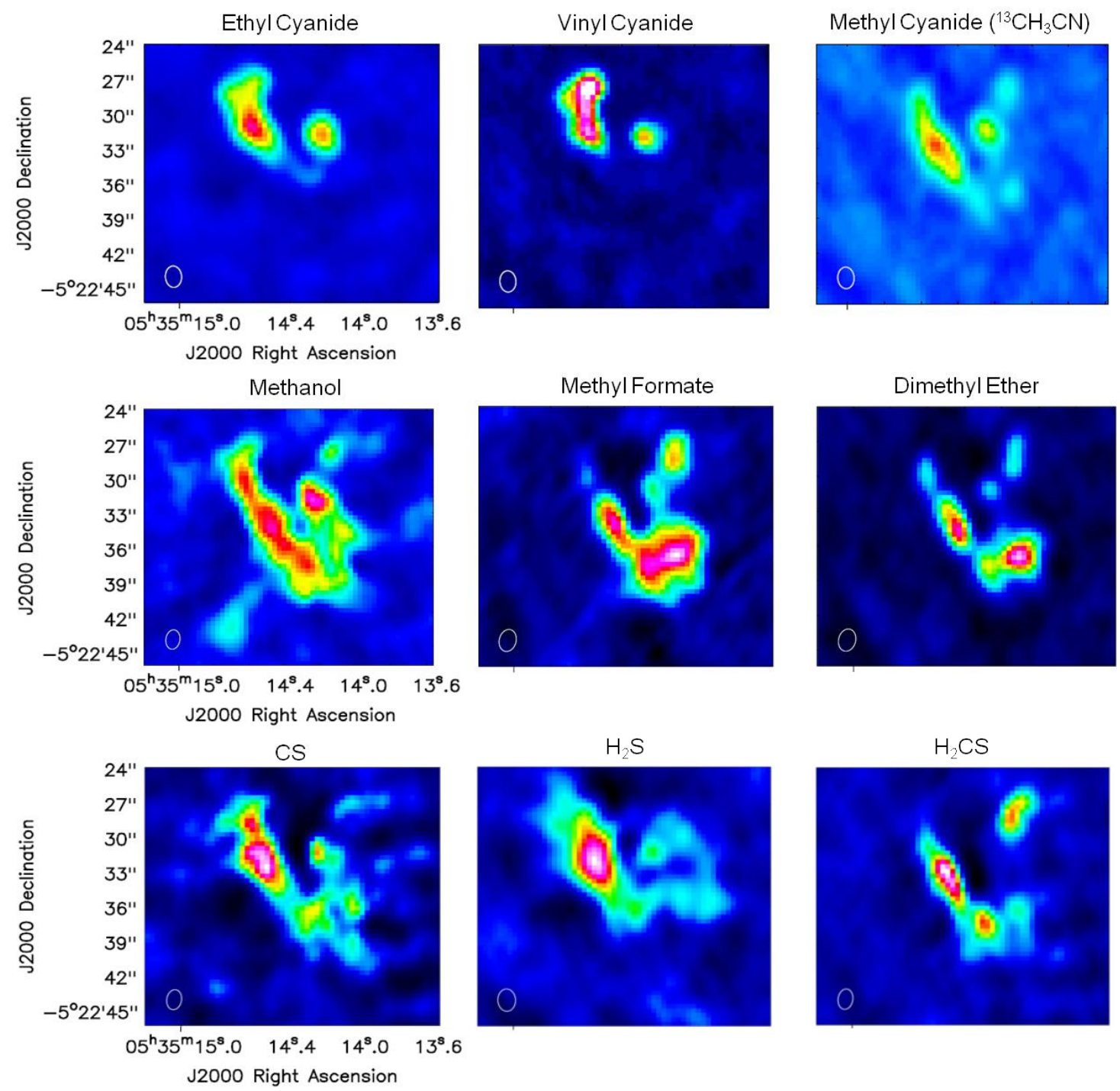

Figure 7.2. Displayed in this figure are the integrated intensity spatial distributions of three distinct chemical "families". The top row shows the spatial distributions of the cyanides, the middle row is the O-bearing species, and the bottom row is the S-bearing species. The transitions displayed for cyanides were the $27_{027}-26_{026}$, the $25_{025}-24_{024}$, and the 26-25 transition for ethyl cyanide, vinyl cyanide and $\mathrm{H}^{13} \mathrm{CCCN}$ respectively. For the oxygen bearing species, the $18_{415}-17_{414}$, the $21_{220}-20_{121}$, and the $12_{48}-11_{47}$ transitions were imaged for methyl fomate, dimethyl either, and acetaldehyde. Finally for the sulfur bearing species, the $5-4$, the $2_{20}-2_{11}$, and the $7_{07}-6_{06}$ transitions are shown for $\mathrm{CS}, \mathrm{H}_{2} \mathrm{~S}$ and $\mathrm{H}_{2} \mathrm{CS}$ respectively 
Besides the presence of different molecular families and their constituents, other molecular signatures can be present. An example of this is seen in Fig. 7.3. A spectrum from a single pixel was taken from the hot core and analyzed for the vibrationally excited states of vinyl cyanide. Vinyl cyanide has been extensively studied ${ }^{24}$, and using the previous data, the ground state and 3 vibrationally excited states were modeled and identified in the hot core spectrum. The line density of vinyl cyanide and its vibrationally excited states takes up approximately $10 \%$ of the spectral lines. The high abundance of transitions due to vinyl cyanide (either the ground state or vibrationally excited states) with other like molecules (ethyl cyanide ${ }^{3}$ ) would create a problem in trying to identify weak molecular species. Other than creating problems in molecular detections, the presence of vibrationally excited states will help with the characterization of different spatial regions. Fig. 7.4 demonstrates this idea. The spatial distribution of the ground state of vinyl cyanide is compared to the distributions of the three vibrationally excited states. The highest energy vibrational state for which there is spectroscopic data, $v_{11}=2$ is $457 \mathrm{~cm}^{-1}$ higher in energy than the ground state ${ }^{24}$. Molecules in this excited state can still be seen throughout most of the hot core; however, the distribution is more compact. An analysis of these vibration states will lead to a more conclusive temperature gradient within a single area.

Due to the complexity, a systematic approach to analyzing the data was needed. This came in an approach much like the broadband reaction screening process that has been shown to be successful ${ }^{6,18,25}$. The spatial distribution screening process was used to look for two weak species that have not been detected in Orion KL, but have been 


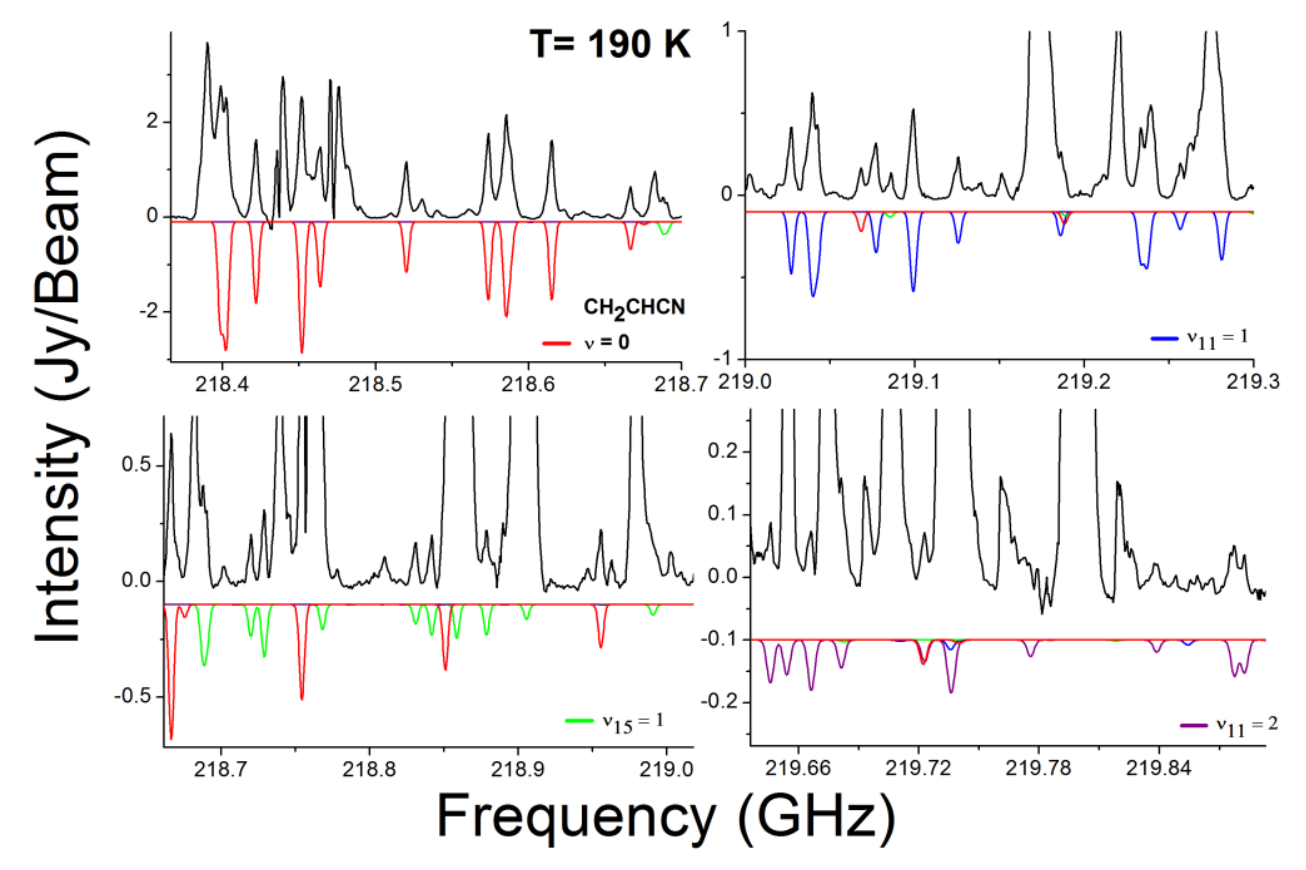

Figure 7.3. The spectrum from the ALMA science verification data was taken over an average of pixels. The pixels used to obtain the spectrum were centered around the pixel at R.A. $=05^{\mathrm{h}} 35^{\mathrm{m}} 14^{\mathrm{s}} .51$, DEC $-05^{\circ} 22^{\prime} 30^{\prime}$.66 and taken over a 2 pixel radius. The simulation of vinyl cyanide $\left(\mathrm{CH}_{2} \mathrm{CHCN}\right)$ was made with a temperature of $190 \mathrm{~K}$ for the ground and excited vibrational states, using previuosly published data ${ }^{24}$. The shown spectral insets display the $\mathrm{J}=23-22$ transitions of the respctive ground or vibrationally excited state. 

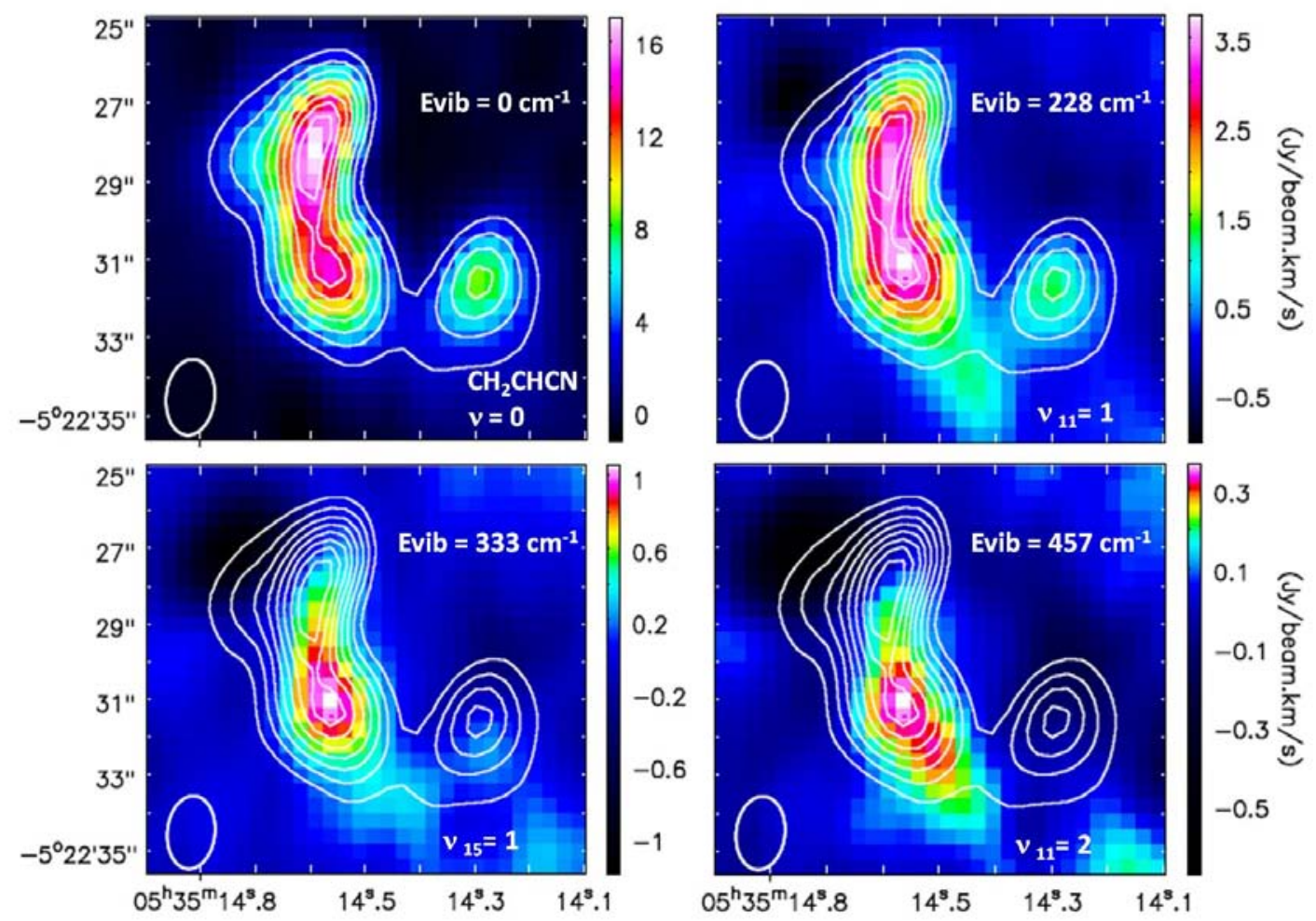

Figure 7.4. Integrated intensity maps of Vinyl Cyanide $\left(\mathrm{J}=23_{419}-22_{418}, \mathrm{E}_{\mathrm{L}}=111.5 \mathrm{~cm}^{-1}\right)$ and its vibrational states. In the upper left hand corner the ground vibrational state is shown, overlapped with its contour representation [contour levels: 3.76,5.64, 7.52, 9.40, $11.28,13.16,15.04,16.92 \mathrm{Jy} / \mathrm{beam}]$. In the subsequent panels, the raster image is of the vibrationally excited states $\left(v_{11}=1\right.$ is $23_{518^{-}}-22_{517}, v_{15}=1$ is $23_{1013^{-}}-22_{1012}, v_{11}=2$ is $23_{1211^{-}}$ $22_{1210}$ ) denoted on the panel, overlaid with the parent contour. 


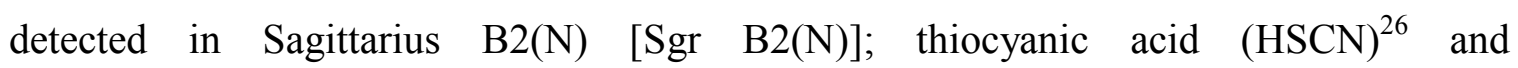
E-cyanomethanimine ${ }^{25}$. HSCN has already been studied up at mm-wave frequencies ${ }^{27}$, and thus accurate line frequencies were available. E-cyanomethanimine is the object of study in Chapter 6 using similar methodology, and thus it was interesting to see if the molecule could be located in Orion KL. The other important factor was that both of these reactions occur through the barrer-less reaction of ${ }^{\circ} \mathrm{CN}$ with the secondary reactant. All three reactants are present in Orion KL. The distribution of ${ }^{\circ} \mathrm{CN}$ can be seen in Fig. 7.5, overlaid with the continuum image from Fig. 7.1, to give a sense of perspective. The ${ }^{\circ} \mathrm{CN}$ resides near the hot core, the NW, and (even though not present on the map) in the extended ridge and also in the plateau. There is evidence from the spatial distribution that ${ }^{\circ} \mathrm{CN}$ is in the southern hot core. The density of the spectrum in that region was prohibitive in trying to obtain a rotational temperature due to blending; however it does appear that the transitions line up with a $5 \mathrm{~km} \mathrm{~s}^{-1}$ velocity shift, which is consistent with other species in the hot core. It is, however, difficult to definitely state that this population of ${ }^{\circ} \mathrm{CN}$ resides in the hot core.

The spatial distribution screening process was first applied to the problem of HSCN. The proposed reaction mechanism for this reaction is seen in Eq. 7.1.

$$
{ }^{\circ} \mathrm{CN}+\mathrm{H}_{2} \mathrm{~S} \rightarrow \mathrm{HSCN}+\mathrm{H}
$$

The raster image of ${ }^{\circ} \mathrm{CN}$ was overlaid with $\mathrm{H}_{2} \mathrm{~S}$, Fig. 7.6, and three pixels were chosen to look for HSCN. The spectra along with a simulation of HSCN are shown in Figs 7.7-9. Velocity shifts were based off of the parameters for $\mathrm{H}_{2} \mathrm{~S}$ as well as the temperature in the 


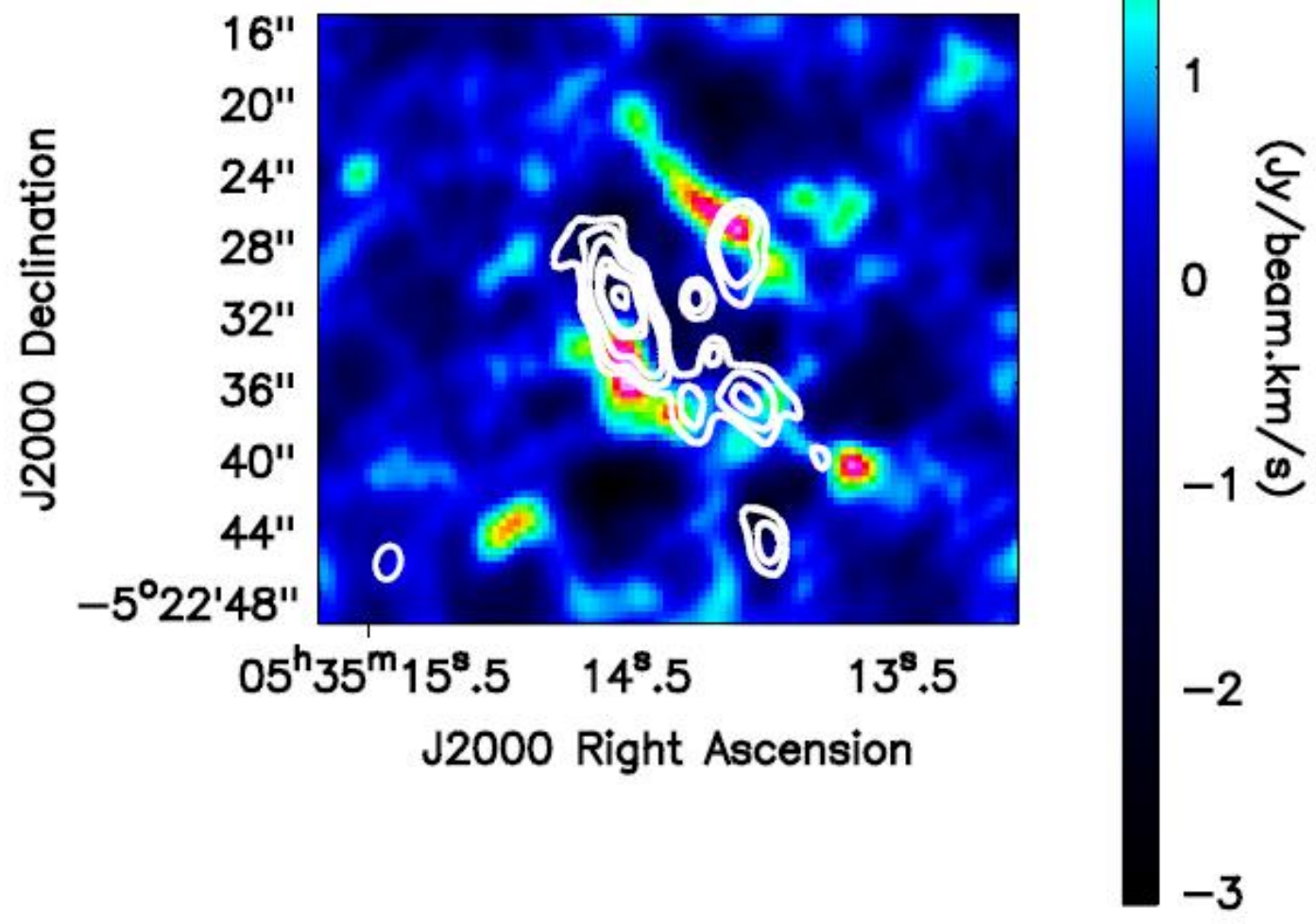

Figure 7.5. The continuum contour image is overlaid with the raster image of the ${ }^{\circ} \mathrm{CN}$. The contour levels are the same as those in Fig. 7.1. The raster image is of the $\mathrm{N}=2-1$ $\mathrm{J}=5 / 2-3 / 2 \mathrm{~F}=5 / 2-3 / 2$ transition of ${ }^{\circ} \mathrm{CN}$ at $226874.166 \mathrm{MHz}$. The distribution of ${ }^{\circ} \mathrm{CN}$ resides mostly near the southern hot core and in the extended ridge. 


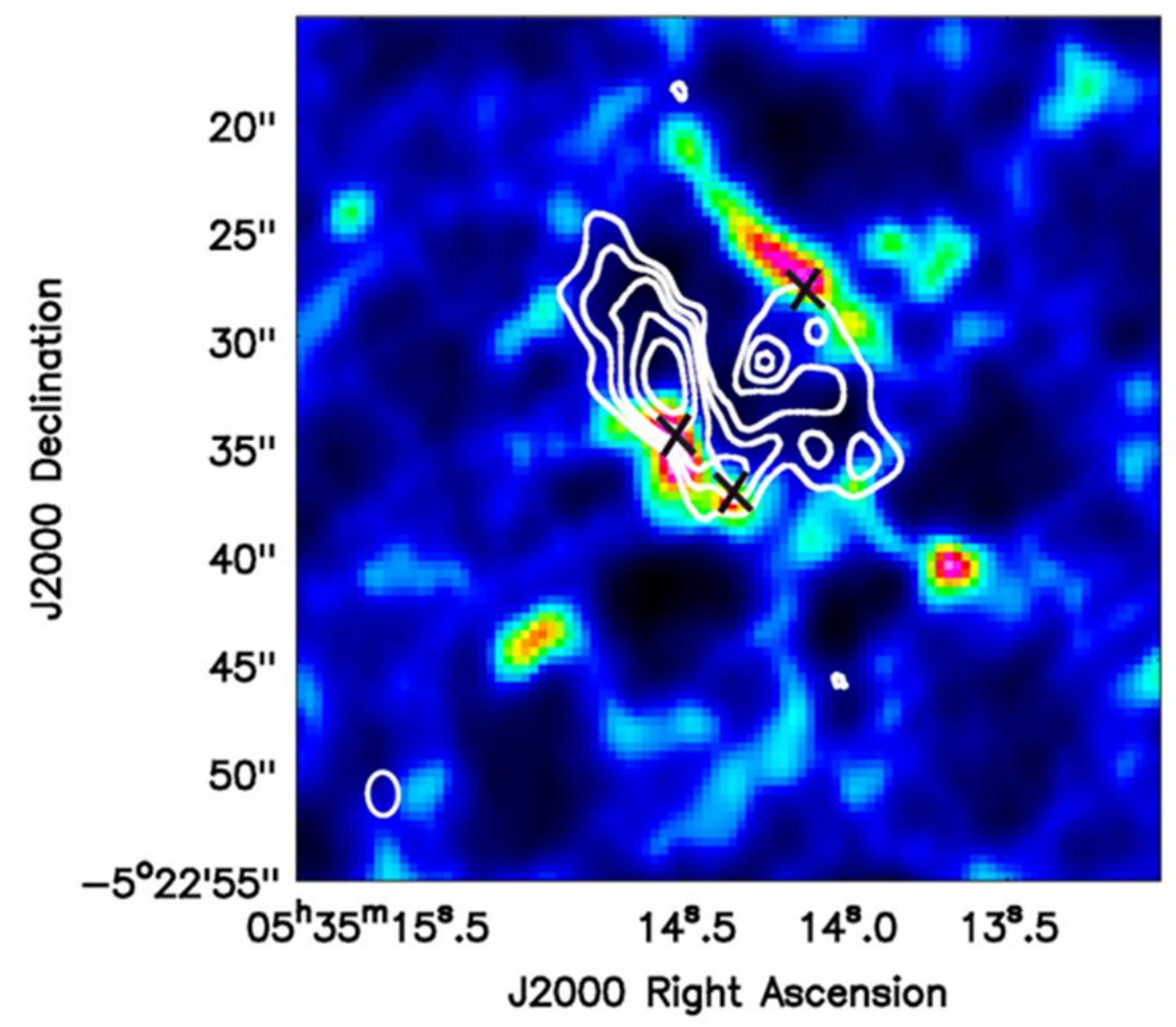

Figure 7.6. This map shows the ${ }^{\circ} \mathrm{CN}$ raster map (from Fig. 7.3) overlaid with the contour map of $\mathrm{H}_{2} \mathrm{~S}$. The black X's denote places of spatial overlap between the two molecules, and this is where the pixel was chosen to look for the reaction product HSCN. The contour levels are 14.32, 21.48, 28.64, 42.96, and 57.28 Jy/beam. The cordinates for the pixel in the southern hot core are R.A. $=05^{\mathrm{h}} 35^{\mathrm{m}} 14^{\mathrm{s}} .511$, DEC $=-05^{\circ} 22^{\prime} 33^{\prime}$ '.909, for the compact ridge region they are R.A. $=05^{\mathrm{h}} 35^{\mathrm{m}} 14^{\mathrm{s}} .342$, DEC $=-05^{\circ} 22^{\prime} 37^{\prime} \cdot .409$ and for the extended ridge in the northwest they are R.A. $=05^{\mathrm{h}} 35^{\mathrm{m}} 14^{\mathrm{s}} .150, \mathrm{DEC}=-05^{\circ} 22^{\prime} 27^{\prime}$ '.998. 


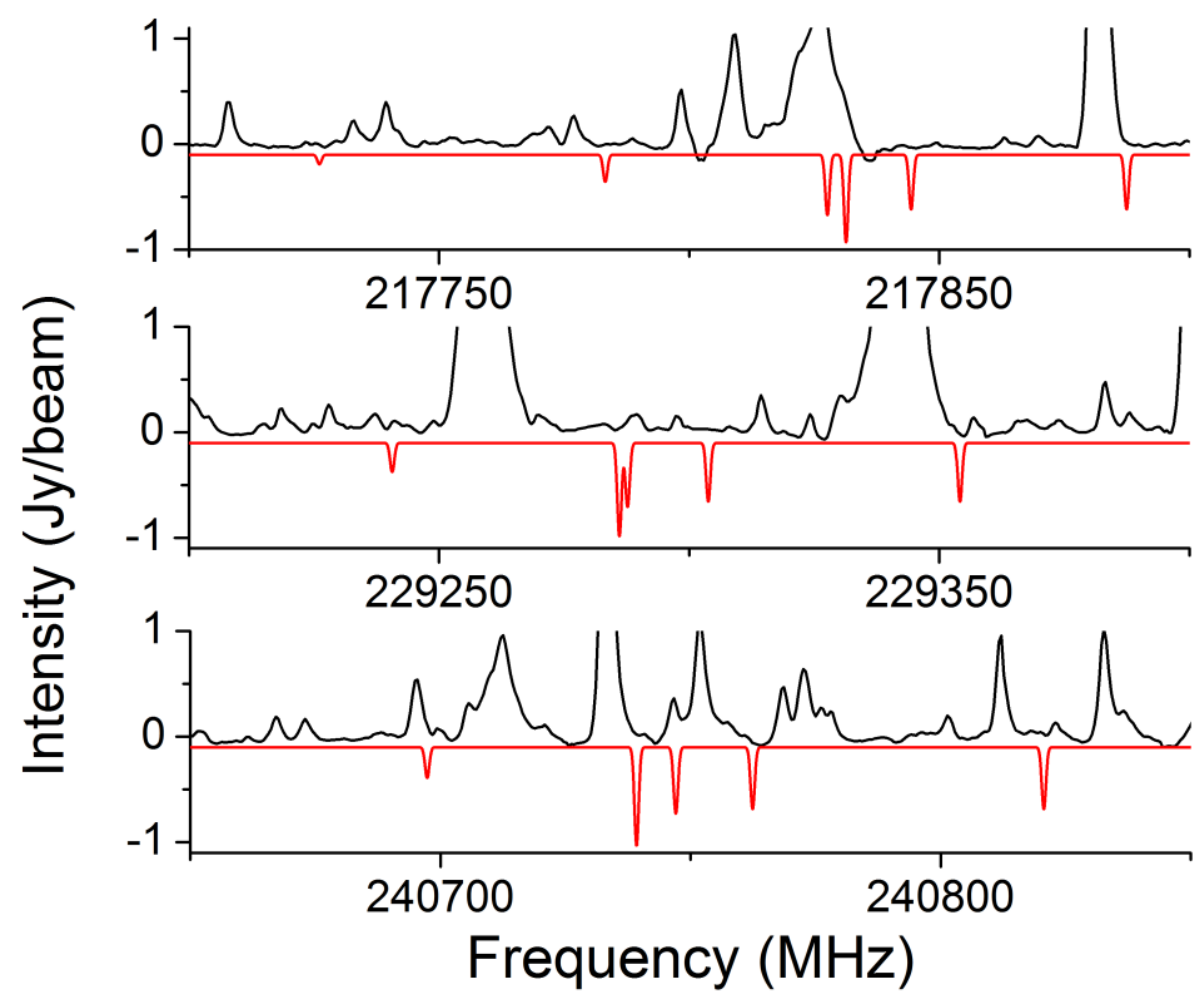

Figure 7.7. A spectrum taken from the hot core at R.A. $=05^{\mathrm{h}} 35^{\mathrm{m}} 14^{\mathrm{s}} \cdot 511$, DEC $=-$ $05^{\circ} 22$ '33". 909 . The black trace shows the spectrum from Orion KL, while the red trace displays the simulated spectrum of $\mathrm{HSCN}^{12}$ at $145 \mathrm{~K}$ and a velocity shift of $4.5 \mathrm{~km} / \mathrm{s}$. The three displayed regions are those for the $J=19-18$ in the upper panel, $J=20-19$ in the middle panel, and $\mathrm{J}=21-20$ in the lower panel. 


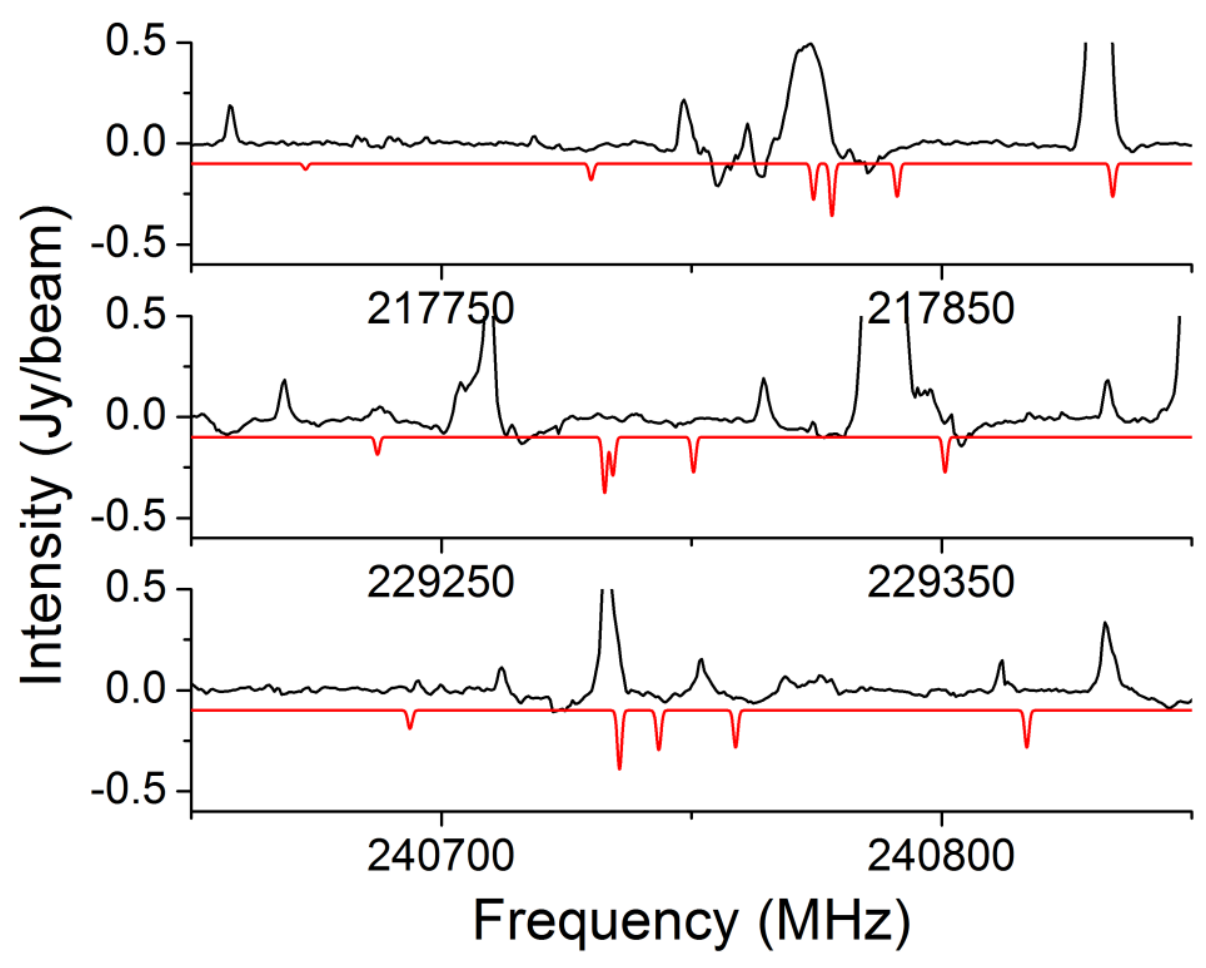

Figure 7.8. This spectrum was taken from the compact ridge region at R.A. = $05^{\mathrm{h}} 35^{\mathrm{m}} 14^{\mathrm{s}} .342, \mathrm{DEC}=-05^{\circ} 22^{\prime} 37^{\prime \prime} .409$. The black trace shows the spectrum from Orion $\mathrm{KL}$, while the red trace displays the simulated spectrum of $\mathrm{HSCN}^{12}$ at $120 \mathrm{~K}$ and a velocity shift of $9 \mathrm{~km} / \mathrm{s}$. The three displayed regions are those for the $\mathrm{J}=19-18$ in the upper panel, $\mathrm{J}=20-19$ in the middle panel, and $\mathrm{J}=21-20$ in the lower panel. 


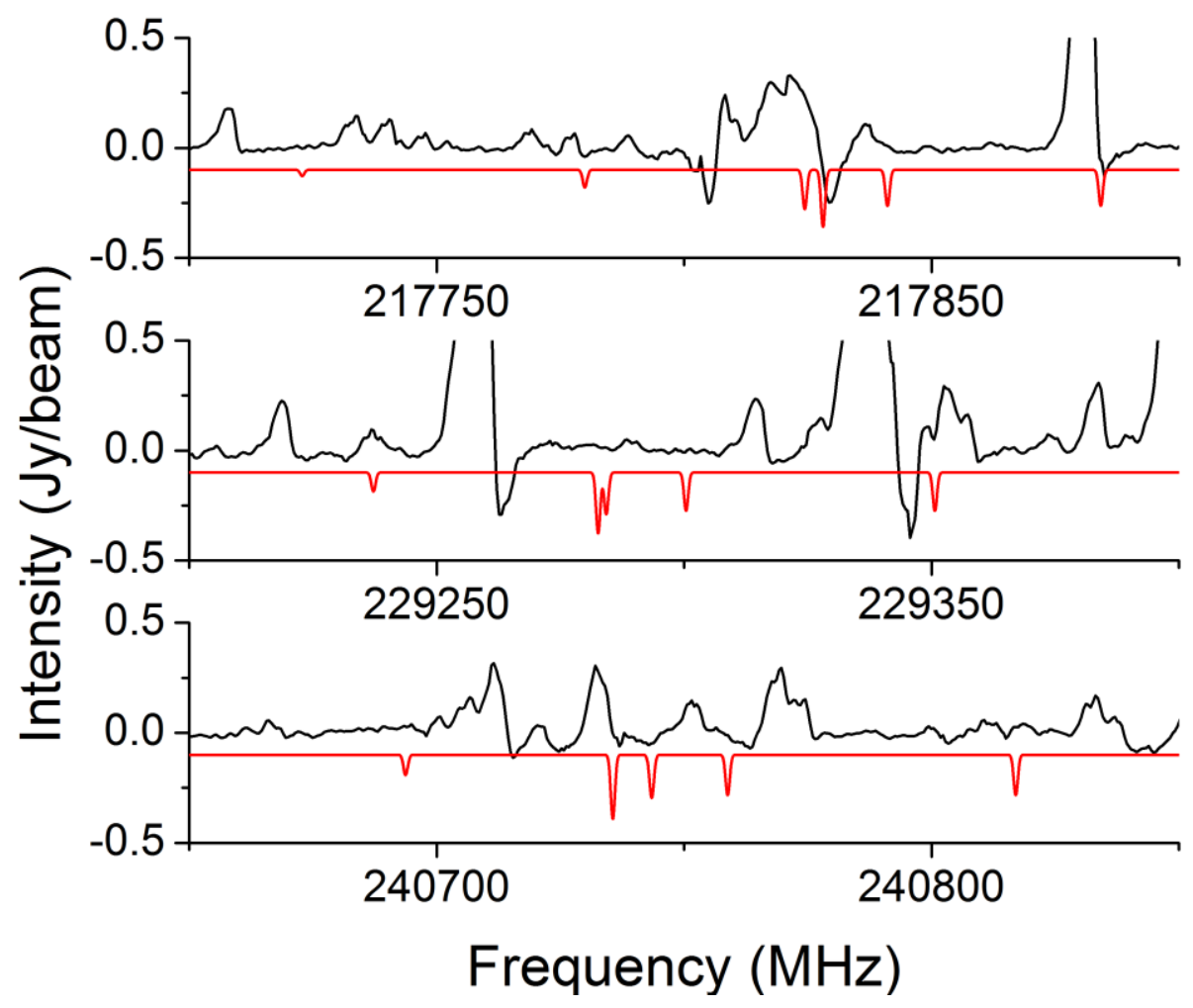

Figure 7.9. A spectrum taken from the extended ridge at R.A. $=05^{\mathrm{h}} 35^{\mathrm{m}} 14^{\mathrm{s}} .150$, DEC $=-$ $05^{\circ} 22$ '27'.998. The black trace shows the spectrum from Orion KL, while the red trace displays the simulated spectrum of $\mathrm{HSCN}^{12}$ at $120 \mathrm{~K}$ and a velocity shift of $9 \mathrm{~km} / \mathrm{s}$. The three displayed regions are those for the $J=19-18$ in the upper panel, $J=20-19$ in the middle panel, and $\mathrm{J}=21-20$ in the lower panel. 
hot core. In the two remaining areas, a variety of temperatures were chosen based on common molecular temperatures in the region. Three transitions per spectrum are shown in each of the figures, and it can be seen that there is no convincing evidence for the presence of HSCN. Other regions of non overlap between the two reactants have also been searched, but it was not detected in these regions either. The second molecule of interest is E-cyanomethanimine, and is proposed to form via the neutral-radical reactionof ${ }^{\circ} \mathrm{CN}$ and methanimine $\left(\mathrm{CH}_{2} \mathrm{NH}\right)$ shown in Eq. 7.2.

$$
\cdot \mathrm{CN}+\mathrm{CH}_{2} \mathrm{NH} \rightarrow \mathrm{HNCHCN}+\mathrm{H}
$$

The spatial distributions of the reactants are shown in Fig. 7.10. Again the raster image shows the distribution of ${ }^{\circ} \mathrm{CN}$, while the contour map displays $\mathrm{CH}_{2} \mathrm{NH}$. Two pixels, one in the hot core and one in the NW, were chosen due to the high level of overlap. The parameters for the simulations in Figs 7.11 and 7.12 of E-cyanomethanimine were chosen based off the parameters for $\mathrm{CH}_{2} \mathrm{NH}$ in Crockett et al. ${ }^{21}$ for the hot core. Again the velocity for the NW was chosen to from that observed for $\mathrm{CH}_{2} \mathrm{NH}$ and a variety of temperatures were fit. Again three a-type transitions were chosen to display and there is no firm evidence for its presence in either region. 


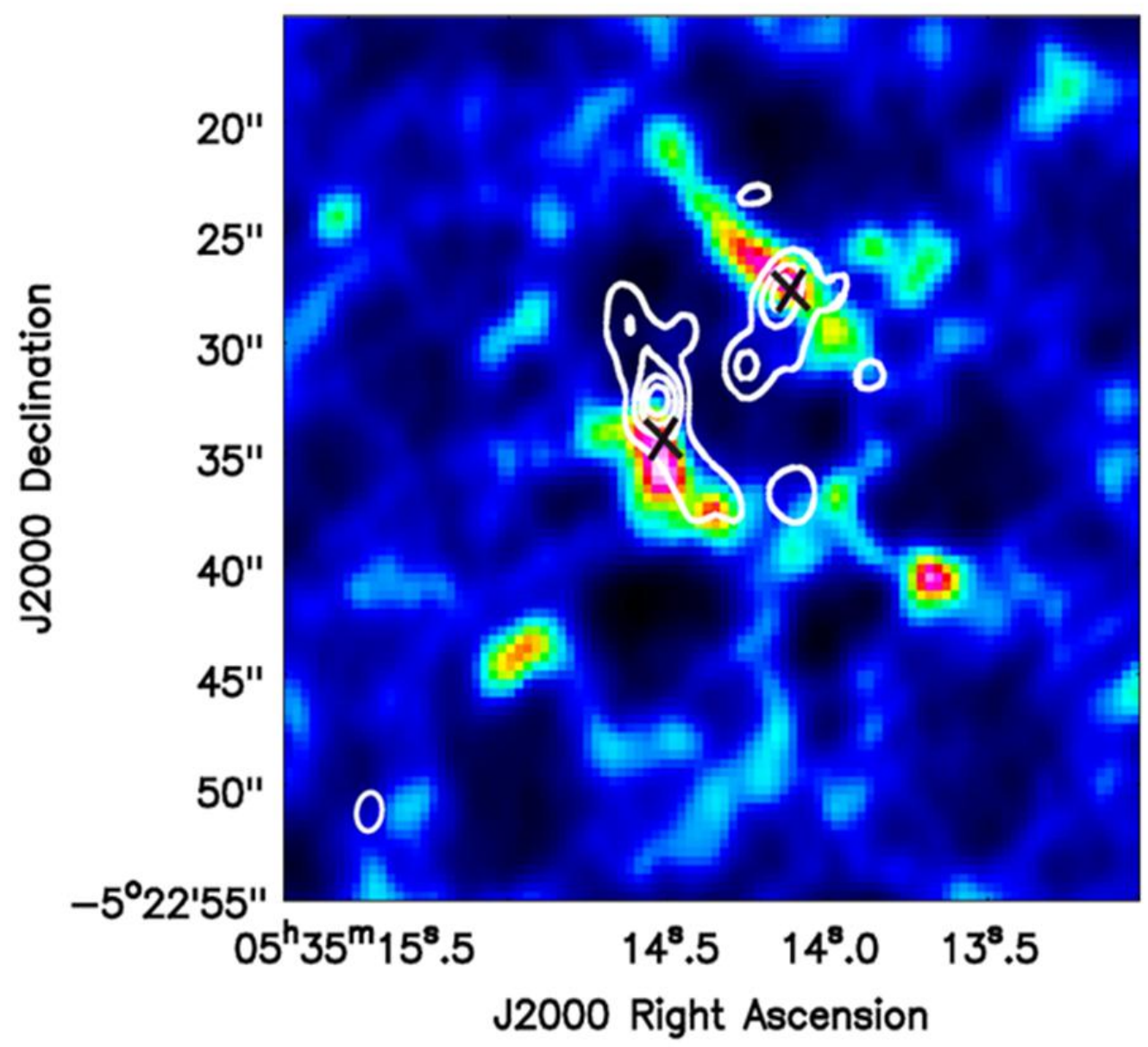

Figure 7.10. The contour map of $\mathrm{CH}_{2} \mathrm{NH}$ overlaid on the raster image of ${ }^{\circ} \mathrm{CN}$ (from Fig. 7.3). The black X's are the places where the spatial distributions of the two molecules overlapped the greatest, and a search for E-cyanomethanimine was conducted. The contour levels are $1.05,2.10,3.15,4.20 \mathrm{Jy} /$ beam. The cordinates for the pixel in the southern hot core are R.A. $=05^{\mathrm{h}} 35^{\mathrm{m}} 14^{\mathrm{s}} .507$, DEC $=-05^{\circ} 22^{\prime} 34^{\prime} .196$ and for the extended ridge in the northwest they are R.A. $=05^{\mathrm{h}} 35^{\mathrm{m}} 14^{\mathrm{s}} .142$, DEC $=-05^{\circ} 22^{\prime} 27^{\prime} .768$. 


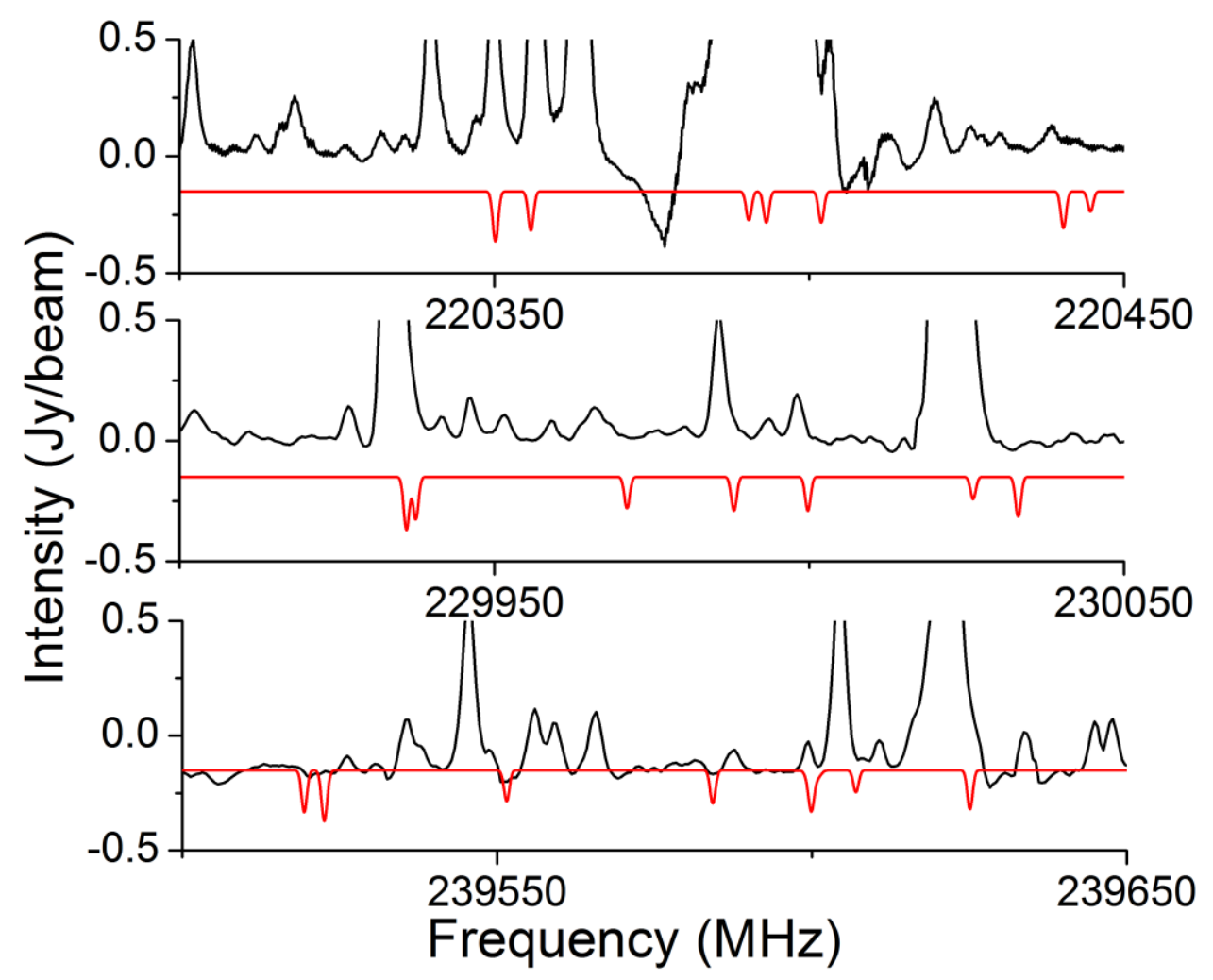

Figure 7.11. The spectrum of Orion KL at R.A. $=05^{\mathrm{h}} 35^{\mathrm{m}} 14^{\mathrm{s}} \cdot 507, \mathrm{DEC}=-05^{\circ} 22^{\prime} 34^{\prime \prime} .196$ in the hot core. A simulated spectrum of E-cyanomethanimine is shown in red at $130 \mathrm{~K}$ and $6 \mathrm{~km} / \mathrm{s}$. The black trace is the experimental trace. Transitions for $\mathrm{J}=23-22$ are shown in the top panel, $\mathrm{J}=24-23$ in the middle panel, and $\mathrm{J}=25-24$ in the bottom panel. 


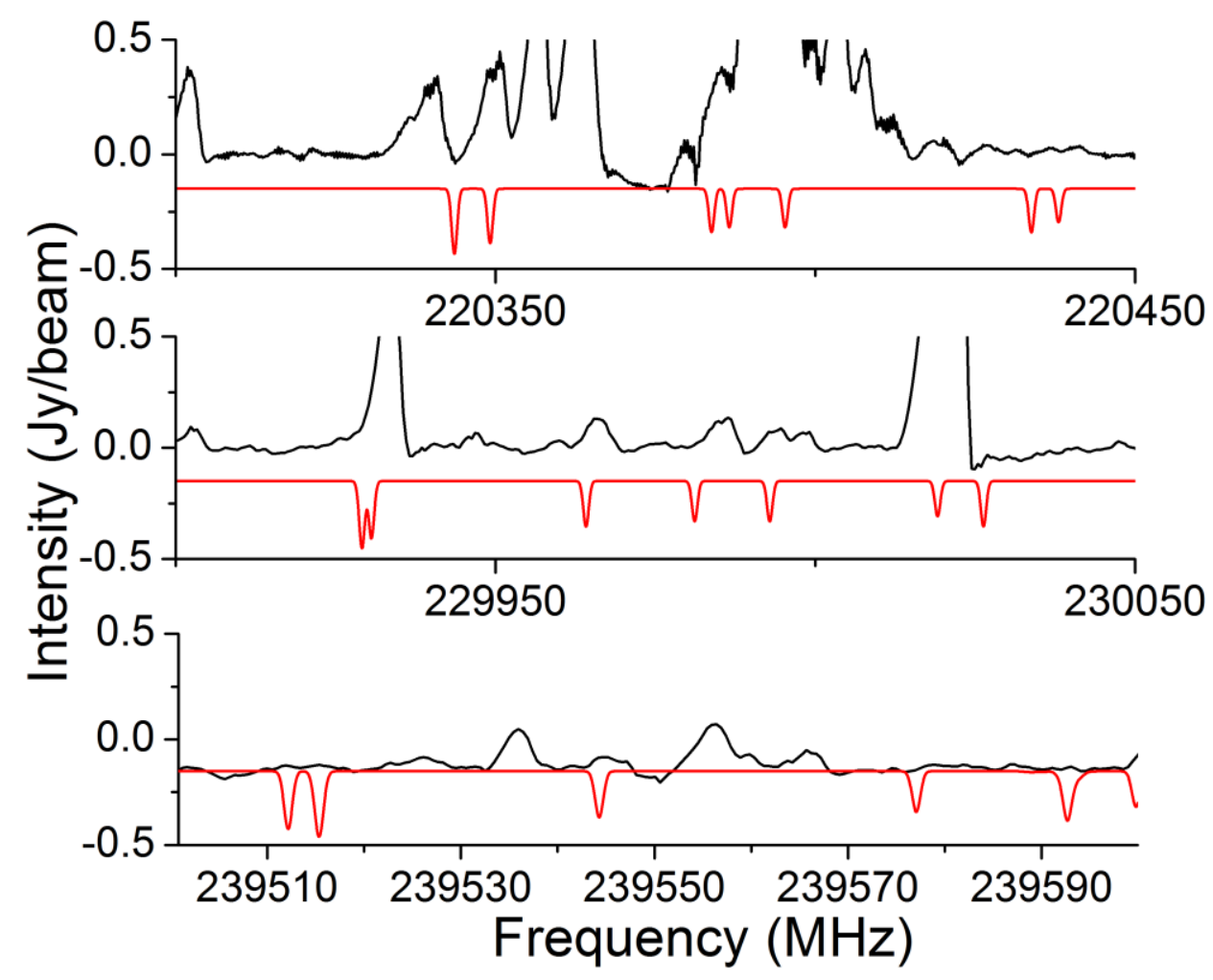

Figure 7.12. The spectrum of Orion KL at R.A. $=05^{\mathrm{h}} 35^{\mathrm{m}} 14^{\mathrm{s}} \cdot 142, \mathrm{DEC}=-05^{\circ} 22^{\prime} 27^{\prime \prime} .768$ in the hot core. A simulated spectrum of E-cyanomethanimine is shown in red at $180 \mathrm{~K}$ and $9 \mathrm{~km} / \mathrm{s}$. The black trace is the experimental trace. Transitions for $\mathrm{J}=23-22$ are shown in the top panel, $\mathrm{J}=24-23$ in the middle panel, and $\mathrm{J}=25-24$ in the bottom panel. 


\section{Discussion}

The molecular distributions can be observed across four distinct regions in Orion KL. Molecules belonging to the cyanide family are found primarily in the hot core region, while the oxygenated species are found in the southern hot core, compact ridge, and NW, which agrees with previous findings ${ }^{16,17,21,23}$. The sulfur bearing species are quite wide spread. These less complex molecules are ubiquitous; however, the number of S-bearing species identified is quite low. As more complex molecules are identified, it highly probable that their spatial distributions will be more compact because they tend to desorb at higher temperatures off of ice grains and require higher temperatures to form. The available distributions indicate that S-bearing species could be interacting with not only oxygenated species, but also the cyanides. A test of this theory comes from the molecular composition of each region. From the HIFI survey ${ }^{21}$, there are not many "mixed" species, or species that could be included in two of the molecular families. The list consists of formamide $\left(\mathrm{NH}_{2} \mathrm{CHO}\right)$, isocyanic acid ( $\mathrm{HNCO}$ ), carbonyl sulfide (OCS), nitrous sulfide (NS), nitrous oxide (NO), and sulfur dioxide $\left(\mathrm{SO}_{2}\right)$. There are several reasons that the mixed complex molecules have not been observed. It also may be that more complex molecules do not exist in these regions, or it may be that there is not the sensitivity to detect weaker (either in abundance or dipole moment), complex molecules. With the high sensitivity of ALMA, this second theory will be tested.

The complexity and depth of the ALMA dataset can make analysis quite difficult. In warm regions, such as the hot core where temperatures can reach 100-300 K, a spectrum from a single pixel is dense, as was seen in Fig. 7.3. The existence of the 
vibrationally excited states of $\mathrm{CH}_{2} \mathrm{CHCN}$ or molecular "weeds" 3 , serve to clutter an already crowded spectrum. The place in which these weeds are preferred is in the assessment of the physical properties of a region like the hot core. The presence of vibrationally excited states gives an idea of the temperatures in the region. The spectra in Fig. 7.3 were modeled at $190 \mathrm{~K}$, which is consistent with the hot core. In order to get around the weeds, automated tools are being developed to help with the assignment of these dense spectra. However, in the case of trying to find new molecular detections, these regions are unfavorable. Therefore, if there are multiple regions of overlap, a preference can be given to the spectra that will occur in places where these spectral weeds will not be as prevalent.

The concept of the spatial distribution screening process was assessed by trying to identify both HSCN and E-cyanomethanimine in the SV dataset. It would be time consuming to go through every pixel to try to find the intended molecule. Thus to increase the chances of success, the screening process was employed. However, both attempts were unsuccessful at identifying the desired product. There has been no evidence from single dish observations towards these sources that these two product molecules would be found, thus indicating that this methodology is/ will not be ineffective. Besides the single dish observations supporting non-detections, there are several reasons the reaction products may not have been seen. When the observations were taken, only 5 minutes of integration was done on each spectral window. This means that only molecules above $10 \mathrm{mJy} /$ beam would be detectable. In the reaction for $\mathrm{E}$ cyanomethanimine, one of the reactants, $\mathrm{CH}_{2} \mathrm{NH}$, only had an integrated intensity of 4.2 
Jy/beam. Depending on the conditions in the regions of overlap and the efficiency of the reaction, E-cyanomethanimine may be present in amounts $<10 \mathrm{mJy} /$ beam. For relatively weak species, more integration time may be needed. The other main problem with the SV dataset is that because only 16 antennas were used for the observation, the UV coverage and sensitivity were not maximized. This left many places were the flux was not accurately measured. The final problem may be that the proposed reactants for the two reactions are not co-spatial, but only appear so from the interferometric maps. While great care was taken to try and ensure that the reactants were co-spatial, regions like the plateau and extended ridge have similar velocity shifts and temperatures. Therefore, it is possible that the reactants aren't co-spatial. In the future, care should be taken when testing this method again. The time consuming nature of this analysis (while faster than previous methods) can be decreased by utilizing the principal component analysis (PCA) method $^{28-31}$. This statistical method can be automated in Python to determine the correlation between the spatial distributions of the two reactants. By using this method, and pairing the spatial distribution screening process with other techniques such as automated spectral assignment and high speed CP-FTmm-wave spectroscopy, the analysis time for these large datasets will be greatly reduced.

\section{Conclusions}

The ALMA datasets that will soon be publicly available will contain large amounts of data that will require months, if not years to analyze. The current approach of 
analyzing each pixel from these datasets based on available spectroscopic databases is both time consuming and unrealistic. In the hunt for new molecular detections, a systematic approach which is guided by chemical understanding and intuition is needed. The spatial distribution screening method is such an approach. When paired with automation techniques, the required time and effort to look for new species will be significantly reduced, and it will take advantage of the spatial information that is now available. The fast spectroscopic techniques, such as the discharge CP-FTmm-wave spectroscopy, will help to increase the throughput of available rotational spectra. This will increase the accuracy of the search. The future for the analysis of these datasets is open, and it is up to the scientific community to determine fast and efficient ways to analyze them. 


\section{Chapter 7 References}

(1) Grabow, J.-U.; Stahl, W.; Dreizler, H. Rev. Sci. Instrum. 1996, 67, 4072-4084.

(2) Thaddeus, P.; McCarthy, M. C. Spectrochim. Acta. A. Mol. Biomol. Spectrosc. 2001, 57, 757-774.

(3) Fortman, S. M.; Medvedev, I. R.; Neese, C. F.; Lucia, F. C. D. Astrophys. J. 2010, $725,1682$.

(4) Steber, A. L.; Harris, B. J.; Neill, J. L.; Pate, B. H. J. Mol. Spectrosc. 2012, 280, 310.

(5) Pearson, J. C.; Drouin, B. J. Astrophys. J. Lett. 2006, 647, L83.

(6) Loomis, R. A.; Zaleski, D. P.; Steber, A. L.; Neill, J. L.; Muckle, M. T.; Harris, B. J.; Hollis, J. M.; Jewell, P. R.; Lattanzi, V.; Lovas, F. J.; Oscar Martinez, J.; McCarthy, M. C.; Remijan, A. J.; Pate, B. H.; Corby, J. F. Astrophys. J. Lett. 2013, 765, L9.

(7) Petkie, D. T.; Goyette, T. M.; Bettens, R. P. A.; Belov, S. P.; Albert, S.; Helminger, P.; Lucia, F. C. D. Rev. Sci. Instrum. 1997, 68, 1675-1683.

(8) Medvedev, I. R.; Neese, C. F.; Plummer, G. M.; De Lucia, F. C. Opt. Lett. 2010, $35,1533-1535$.

(9) Harris, B. J. A Chirped Pulse Fourier Transform Millimeter Wave Spectrometer for Room Temperature, Gas Mixture Analysis. Doctor of Philosophy, University of Virginia, 2014.

(10) Remijan, A. J. Splatalogue database for astronomical spectroscopy http://www.cv.nrao.edu/php/splat/ (accessed Oct 26, 2014). 
(11) Müller, H. S. P.; Schlöder, F.; Stutzki, J.; Winnewisser, G. J. Mol. Struct. 2005, $742,215-227$.

(12) Pickett, H. M.; Poynter, R. L.; Cohen, E. A.; Delitsky, M. L.; Pearson, J. C.; Müller, H. S. P. J. Quant. Spectrosc. Radiat. Transf. 1998, 60, 883-890.

(13) Woon, D. E. The Astrochymist http://astrochymist.org/astrochymist_ism.html (accessed Oct 1, 2014).

(14) Belloche, A.; Garrod, R. T.; Müller, H. S. P.; Menten, K. M. Science 2014, 345, $1584-1587$.

(15) Wakelam, V.; Smith, I. W. M.; Herbst, E.; Troe, J.; Geppert, W.; Linnartz, H.; Öberg, K.; Roueff, E.; Agúndez, M.; Pernot, P.; Cuppen, H. M.; Loison, J. C.; Talbi, D. Space Sci. Rev. 2010, 156, 13-72.

(16) Friedel, D. N.; Snyder, L. E. Astrophys. J. 2008, 672, 962-973.

(17) Millar, T. J.; Herbst, E.; Charnley, S. B. Astrophys. J. 1991, 369, 147-156.

(18) Neill, J. L.; Muckle, M. T.; Zaleski, D. P.; Steber, A. L.; Pate, B. H.; Lattanzi, V.; Spezzano, S.; McCarthy, M. C.; Remijan, A. J. Astrophys. J. 2012, 755, 153.

(19) Zaleski, D. P.; Stephens, S. L.; Walker, N. R. Phys. Chem. Chem. Phys. 2014.

(20) Common Astronomy Software Application; National Radio Astronomy Observatory.

(21) Crockett, N. R.; Bergin, E. A.; Neill, J. L.; Favre, C.; Schilke, P.; Lis, D. C.; Bell, T. A.; Blake, G.; Cernicharo, J.; Emprechtinger, M.; Esplugues, G. B.; Gupta, H.; Kleshcheva, M.; Lord, S.; Marcelino, N.; McGuire, B. A.; Pearson, J.; Phillips, T. G.; Plume, R.; Tak, F. van der; Tercero, B.; Yu, S. Astrophys. J. 2014, 787, 112. 
(22) Favre, C.; Despois, D.; Brouillet, N.; Baudry, A.; Combes, F.; Guélin, M.; Wootten, A.; Wlodarczak, G. Astron. Astrophys. 2011, 532, A32.

(23) Blake, G. A.; Sutton, E. C.; Masson, C. R.; Phillips, T. G. Astrophys. J. 1987, 315, $621-645$.

(24) Kisiel, Z.; Pszczółkowski, L.; Drouin, B. J.; Brauer, C. S.; Yu, S.; Pearson, J. C.; Medvedev, I. R.; Fortman, S.; Neese, C. J. Mol. Spectrosc. 2012, 280, 134-144.

(25) Zaleski, D. P.; Seifert, N. A.; Steber, A. L.; Muckle, M. T.; Loomis, R. A.; Corby, J. F.; Oscar Martinez, J.; Crabtree, K. N.; Jewell, P. R.; Hollis, J. M.; Lovas, F. J.; Vasquez, D.; Nyiramahirwe, J.; Sciortino, N.; Johnson, K.; McCarthy, M. C.; Remijan, A. J.; Pate, B. H. Astrophys. J. Lett. 2013, 765, L10.

(26) Halfen, D. T.; Ziurys, L. M.; Brünken, S.; Gottlieb, C. A.; McCarthy, M. C.; Thaddeus, P. Astrophys. J. Lett. 2009, 702, L124.

(27) Brünken, S.; Yu, Z.; Gottlieb, C. A.; McCarthy, M. C.; Thaddeus, P. Astrophys. J. 2009, 706, 1588-1593.

(28) Jones, P. A.; Burton, M. G.; Tothill, N. F. H.; Cunningham, M. R. Mon. Not. R. Astron. Soc. 2011, 411, 2293-2310.

(29) Lo, N.; Cunningham, M. R.; Jones, P. A.; Bains, I.; Burton, M. G.; Wong, T.; Muller, E.; Kramer, C.; Ossenkopf, V.; Henkel, C.; Deragopian, G.; Donnelly, S.; Ladd, E. F. Mon. Not. R. Astron. Soc. 2009, 395, 1021-1042.

(30) Ungerechts, H.; Bergin, E. A.; Goldsmith, P. F.; Irvine, W. M.; Schloerb, F. P.; Snell, R. L. Astrophys. J. 1997, 482, 245-266.

(31) Heyer, M. H.; Peter Schloerb, F. Astrophys. J. 1997, 475, 173-187. 


\section{Chapter 8}

\section{Conclusions}

\section{Summary}

This thesis has presented the design and implementation of several instruments, as well as some of their possible astrochemical and analytical applications. The development of the CP-FTmm-wave instrument allowed for the first CP-FTmm-wave spectrum in the $260-295 \mathrm{GHz}$ region to be taken and matched up against millimeter wave astronomical observations such as those from ALMA. However, the instrument in the full band configuration could not efficiently exploit its other ability of analytical application. Thus modifications were made, and low cost MMIC components were used to be able to perform targeted searches as well as the characterization of molecules, either known or unknown. A similar instrument was built at $18-26.5 \mathrm{GHz}$ and can be used to characterize a molecule or to help identify molecular species in the GBT PRIMOS ${ }^{1}$ survey. The astronomical applications of this instrument will be fully utilized as a key piece in a museum exhibit in which the public will learn about astrochemistry. Utilizing the mmwave instrument and some of the techniques from the previously developed CP-FTMW discharge spectrometer, the segmented CP-FTmm-wave instrument was shown to have the further astronomical capabilities not only in measuring stable compounds that can be purchased, but also and more importantly those made from reactive chemistry within a discharge experiment. This suite of instruments allows for the advancement of 
astrochemistry as well as expands the applicability of rotational spectroscopy as an analytical tool.

The development of techniques to analyze the new data sets coming from both the VLA and ALMA has also been presented. Both instruments offer high spatial resolution, sensitivity, and spectral resolution. The spatial information available from both interferometers is valuable to our understanding of star forming regions. We can begin to understand the chemical composition of these dense molecular clouds and be able to monitor and shed light in the reaction networks in a more accurate fashion. The spatial distribution screening method takes chemical intuition and information and tries to apply it to the chemistry that could be occurring in the ISM. In both the cases of Ecyanomethanimine in Sgr B2(N) and B2(M) and HSCN and E-cyanomethanimine in Orion KL, this method was used and was mildly successful in Sagittarius, while further testing is need in complete ALMA datasets. When paired with fast laboratory instruments such as the segmented CP-FTmm-wave instrument and automated assignment tools, this technique could be quite powerful.

\section{Future developments}

By utilizing the instruments that have been developed, and implementing some key changes, the sensitivity and measurement speed of the described instruments can be used to aid not only astrochemists, but also help bring rotational spectroscopy into the forefront as an analytical tool. By reducing the size and the cost of the mm-wave instrument, commercial grade instruments could be on the market within a matter of 
years. For the microwave spectrometer, an increase in sensitivity would need to be achieved; however exploiting the idea of superradiance, a new instrument design could be drawn up. It would take advantage of the reduced power spectral density and increased volume of sample. The discharge instrument could be modified so that the real time capabilities of the digitizer could be used to monitor reactive species.

The ability to automate many of the tedious tasks that have historically been done by hand in rotational spectroscopy will allow for the faster throughput of data, especially large data sets. Instruments can be programmed with easy to use scripting languages such as Python to "remote" control an instrument as seen in Chapter 4. This would help to remove user error when setting up an instrument and also in the transfer of data. The data could then be worked up immediately and presented to the user through an easy to use graphical user interface. By pairing this with the segmented CP-FTmm-wave instrument, a high fidelity spectrum with dynamic range of $>10^{6}$ would be possible in less than 1.5 minutes. Automated spectral assignments from stored libraries would create an experience for the user, in which a question was asked and answer in a matter of minutes with minimal effort. Applying this technique to either an analytical application or astrochemical application would be extremely straightforward.

The ability to work with the large data sets coming from ALMA and the VLA is crucial to the future of the astrochemical field. This relies not only on the speed and fidelity of the laboratory data that will and has been produced, but also with the way in which analyze the datasets. While not completely successful in the course of this thesis, the spatial distribution screening method does have the promise of being one of the 
methods in which the dense and high resolution interferometric data can be analyzed in a short period of time. Reactions for chemical species have been proposed in the literature for decades ${ }^{2-5}$. Taking advantage of these reaction networks, areas in which these molecules were previously unidentified can be probed again and searched. This method along with the discussed instruments will play a key role in furthering our understanding of complex molecular clouds. 


\section{Chapter 8 References}

(1) Remijan, A. J.; Hollis, J. M.; Jewell, P. R.; Lovas, F.; Corby, J. 2013; Vol. 221.

(2) Petrie, S.; Herbst, E. Astrophys. J. 1997, 491, 210.

(3) Prasad, S. S.; Huntress, W. T., Jr. Astrophys. J. Suppl. Ser. 1980, 43, 1-35.

(4) Wakelam, V.; Smith, I. W. M.; Herbst, E.; Troe, J.; Geppert, W.; Linnartz, H.;

Öberg, K.; Roueff, E.; Agúndez, M.; Pernot, P.; Cuppen, H. M.; Loison, J. C.; Talbi, D. Space Sci. Rev. 2010, 156, 13-72.

(5) Watson, W. D. Rev. Mod. Phys. 1976, 48, 513-552. 